\title{
Hepatosplanchnic dysfunction in sepsis and multiple organ failure: role of haemodynamic and inflammatory disturbances
}

Citation for published version (APA):

Poeze, M. (2004). Hepatosplanchnic dysfunction in sepsis and multiple organ failure: role of haemodynamic and inflammatory disturbances. [Doctoral Thesis, Maastricht University]. Maastricht University. https://doi.org/10.26481/dis.20040611mp

Document status and date:

Published: 01/01/2004

DOI:

10.26481/dis.20040611mp

Document Version:

Publisher's PDF, also known as Version of record

Please check the document version of this publication:

- A submitted manuscript is the version of the article upon submission and before peer-review. There can be important differences between the submitted version and the official published version of record. People interested in the research are advised to contact the author for the final version of the publication, or visit the DOI to the publisher's website.

- The final author version and the galley proof are versions of the publication after peer review.

- The final published version features the final layout of the paper including the volume, issue and page numbers.

Link to publication

\footnotetext{
General rights rights.

- You may freely distribute the URL identifying the publication in the public portal. please follow below link for the End User Agreement:

www.umlib.nl/taverne-license

Take down policy

If you believe that this document breaches copyright please contact us at:

repository@maastrichtuniversity.nl

providing details and we will investigate your claim.
}

Copyright and moral rights for the publications made accessible in the public portal are retained by the authors and/or other copyright owners and it is a condition of accessing publications that users recognise and abide by the legal requirements associated with these

- Users may download and print one copy of any publication from the public portal for the purpose of private study or research.

- You may not further distribute the material or use it for any profit-making activity or commercial gain

If the publication is distributed under the terms of Article $25 \mathrm{fa}$ of the Dutch Copyright Act, indicated by the "Taverne" license above, 
Hepatosplanchnic dysfunction in sepsis and multiple organ failure

Role of haemodynamic

and inflammatory disturbances

Martijn Poeze 


\section{Hepatosplanchnic dysfunction in sepsis and multiple organ failure}

Role of haemodynamic

and inflammatory disturbances

PROEFSCHRIFT

ter verkrijging van de graad van doctor

aan de Universiteit Maastricht, op gezag van de Rector Magnificus,

Prof. mr. G.P.M.F. Mols

volgens het besluit van het College van Decanen

in het openbaar te verdedigen

op vrijdag 11 juni 2004 om 14:00

door

Martijn Poeze

Geboren op 11 augustus 1971 te Muiden 


\section{Promotor}

Prof. Dr. C. Ramsay

\section{Co-promotor}

Dr. J.W.M. Greve

\section{Beoordelingscommissie}

Prof. Dr. K. Leunissen (voorzitter)

Dr. M. Marcus

Prof. Dr. P. Radermacher (Universitaet UIm)

Prof. Dr. M. Singer (University College London)

\section{Colofon}

Vormgeving: Nora Oosting \& Leo Zander

Printing: Unigraphic, Maastricht

ISBN 90-808752-1-x 


\section{LIST OF ABBREVIATIONS}

\begin{tabular}{|c|c|}
\hline ARDS & Adult respiratory distress syndrome \\
\hline $\mathrm{CO}$ & Cardiac output \\
\hline $\mathrm{Cl}$ & Cardiac index \\
\hline $\mathrm{DO}_{2}$ & Oxygen delivery \\
\hline ELISA & Enzyme linker immunosorbent assay \\
\hline FTc & Flow time corrected for heart rate \\
\hline GEDV & Gilobal end-diastolic volume \\
\hline HR & Heart rate \\
\hline ICAM-1 & Intercellular adhesion molecule-1 \\
\hline IL & Interleukin \\
\hline LPS & Lipopolysaccharide \\
\hline LVSW" & Left ventricular stroke work index \\
\hline $\mathrm{mAb}$ & monoclonal antibody \\
\hline MAP & Mean arterial pressure \\
\hline MODS & Multiple organ dysfunction symdrome \\
\hline $\mathrm{MOF}$ & Multiple organ failure \\
\hline $\mathrm{NO}$ & Nitric oxide \\
\hline NOS & NO synthase \\
\hline $\mathrm{O}_{2} \mathrm{ER}$ & Oxygen extraction rate \\
\hline $\mathrm{Pg}$-etCO $\mathrm{CO}_{2}$ gap & $\begin{array}{l}\text { Difference between (gastric) mucosal and end-tidal carbon dioxide } \\
\text { pressure }\end{array}$ \\
\hline $\mathrm{PgaCO}_{2}$ gap & $\begin{array}{l}\text { Difference between (gastric) mucosal and arterial blood carbon } \\
\text { dioxide pressure }\end{array}$ \\
\hline PCT & Procalcithonin \\
\hline $\mathrm{pHi}$ & Intramucosal pH \\
\hline $\mathrm{R}$ & Receptor \\
\hline RHEDV & Right heart end-diastolic volume \\
\hline RVEDV & Right ventricle end-diastolic volume \\
\hline RVEF & Right ventricle ejection fraction \\
\hline s & Soluble \\
\hline SIRS & Systemic inflammatory response syndrome \\
\hline SV & Stroke volume \\
\hline TNF & Tumour necrosis factor \\
\hline VAP & Ventilator-associated pneumonia \\
\hline $\mathrm{VO}_{2}$ & Oxygen consumption \\
\hline
\end{tabular}


Nothing has happened until it has been recorded

Virginia Woolf 


\section{CONTENTS}

\section{Chapter}

1. General introduction

2. Review of monitoring intensive care patients: global and regional aspects

3. Outline of thesis

4. Prediction of postoperative cardiac surgical morbidity and organ failure within 4 hours of icu admission using oesophageal doppler ultrasonography

5. Effect of dopexamine on outcome after major abdominal surgery: a prospective, randomised, controlled multi-centre study

6. A meta-analysis of haemodynamic optimisation of perioperative patients and patients with sepsis: relationship with the methodological trial quality

7. Monitoring global or regional haemodynamic parameters in critically ill patients: what is more important during intensive care resuscitation?

8. D-lactate as an early marker of intestinal ischaemia after abdominal aneurysm repair

9. Gastric $\mathrm{PgCO}_{2}$ and $\mathrm{Pg}-\mathrm{aCO}$ gap are related to $\mathrm{D}$-llactate and not to L-lactate levels in patients with septic shock

10. Pre-operative tonometry is predictive for mortality and morbidity in high-risk surgical patients

11. Increased hepatosplanchnic inflammation precedes the development of organ failure after elective high-risk surgery

12. Prolonged nitric oxide synthase-2 inhibition during hyperdynamic endotoxemia in the pig increases mortality and deteriorates hepatosplanchnic perfusion and metabolism

13. Beneficial effects of L-arginine on nitric oxide metabolism and hepatosplanchnic perfusion during porcine endotoxemia

14. General discussion and summary

15. Samenvatting in het Nederlands

List of publications

Dankwoord

Curriculum vitae 


\section{Published in}

G Ramsay, M Poeze, IS Grant, C Wallis, A Cushieri. Management of critically ill patients. In: A Cushieri, RJC Steele, AR Moossa (eds.). Essential surgical practice. 2000, Butterworth Heinemann, Oxford, module 19, pp. 427-456.

M Poeze, JWM Greve, G Ramsay. Gut decontamination prevention of translocation. In: AE Baue, DE Fry (eds). SIRS, MODS and MOF - Systemic inflammatory response syndrome, multiple organ dysfunction syndrome and multiple organ failure - pathophysiology, prevention and therapy. 2000, Springer Verlag, Berlin, 1st edition, chapter 59, pp. 580-590.

$M$ Poeze, JWM Greve, G Ramsay. Is splanchnic perfusion critical in sepsis? In: AE Baue, G Berlot, A Gullo, J-L Vincent (eds), Sepsis and organ dysfunction. From basics to clinical approach. 1998, Springer-Verlag, Berlin, $1^{\text {st }}$ ed., ch. 17, pp.169-180. 


\section{SUMMARY}

Despite the improved ability to treat dysfunction of individual organs, the treatment of sequentially failing organs remains difficult, and there is a need for a better understanding of the underlying mechanisms. Recent data have revealed a considerable number of pathophysiological mechanisms for the development of sustained organ fallure and sepsis, In this process of sequentially failing organs, disturbances of perfusion and inflammation seem to be particularly important.

10 Relative or absolute perfusion disturbances, at a macrocirculatory as well as microcirculatory level, may be abnormalities related to an exaggerated regional and systemic inflammatory response. This inflammatory response may, be initiated from different organs, although the gut and liver have been identified as important regulators of this process in both circumstances. 


\section{HISTORY OF ORGAN FAILURE FOLLOWING SHOCK}

In the past mortality in patients with traumatic wounds was high. Around 2000 years ago Celsus described a case of traumatic haemorrhage: "when the heart is wounded, much blood issues, pulse fades away, the colour of the skin becomes extremely pallid, a cold and malodorous sweat suddenly covers the body, the extremitites turn cold and death quickly follows'. However, it was not until 1917 that it was recognised that this mortality was caused by the loss of circulating volume $(1,2)$. This led to the use of fluid interventions aimed at reversing blood loss during the World War I. Another aspect of this fluid resuscitation was pointed out by Cannon et al., who warned in 1918 that, 'Injection of a fluid that will increase blood pressure has dangers in itself'. Haemorrhage in a case of shock may not have occurred to a marked degree because pressure has been too low and the flow too scant to overcome the obstacle offered by a clot. If the pressure is raised before the surgeon is ready to check any bleeding that may take place, blood that is sorely needed may be lost'. It was recognised that patients frequently died even though the bleeding could be stopped and corrected. This was related to a condition first described as metabolic acidosis, for which treatment with bicarbonate was first instituted during World War I.

The next problem encountered in patients who were successfully resuscitated was a new clinical syndrome producing a high level of mortality (post-traumatic renal failure), a condition which is now known as acute tubular necrosis. The occurrence of post-traumatic renal failure is related to the application of massive blood transfusions and the duration of shock. Emphasis in shock resuscitation during the Korean War was on fluid resuscitation and rapid initiation of this fluid resuscitation, which led to a reduction in mortality.

The next problem to solve during shock resuscitation was that patients would frequently suffer respiratory distress. During the Vietnam war, major advances in critical care were made in treating multiple-trauma victims. Advances in pulmonary support, using ventilators, considerably improved survival from respiratory failure. The 1970 s and 1980 s saw new major advances in organ support therapies, such as dialysis and the use of vasoactive drugs, lowering mortality rates among these patients even further.

However, in the present intensive care era, a new syndrome seems to be emerging, which may prove to be one of the most difficult hurdles to take. The occurrence of so-called multiple organ failure (MOF), during which, several organs fail, often in sequential order, was recognised only a few decades ago. Despite the advances in organ support therapies, it has so far proved difficult to obtain significant reductions in mortality. Recent studies have investigated the pathophysiology of MOF in an effort to find new therapeutic options. In order to assist clinical research among MOF patients, attempts have been made to formulate accurate and concise definitions of MOF and related syndromes. 


\section{TERMINOLOGY OF MULTIPLE ORGAN FAILURE (MOF) AND RELATED SYNDROMES}

\section{Multiple organ failure syndrome}

The terms progressive or sequential organ failure (3), multiple organ failure (MOF)(4) and multiple systems organ failure have been introduced to describe an evolving clinical syndrome characterised by the development of otherwise unexplained abnormalities of organ function in critically ill patients. Conventional terminology is considered inadequate to accurately characterise this syndrome, and the clinical description of MOF was made arbitrarily and retrospectively (4). The features of the syndrome have been well described. In most patients, multiple organ failure follows a predictable course, generally beginning at the lungs and progressing to the liver, gastrointestinal tract and kidneys. Bone marrow and cardiac failure are late features of MOF. It must be emphasised, however, that the first organ to fail may depend on the primary diseased organ. Respiratory failure may be the first feature of the syndrome in patients with pneumonia, whereas cardiac failure may be the first feature in patients after coronary bypass surgery for myocardial infarction.

Generally speaking, multiple organ dysfunction syndrome (MODS) refers to a condition in which an acutely ill patient has an altered organ function such that homeostasis cannot be maintained without intervention in more than two organs (5). Criteria for defining abnormalities in a specific organ function have, for the most part, been predicated upon the concept of organ failure, a dichotomous event that is either present or absent (see MOF score by Goris et al.) (4), or on that of organ dysfunction, a continuum of physiologic derangements (multiple organ dysfunction syndrome) (see SOFA score by Vincent et al.)(3).

\section{Sepsis}

In discussing the multiple organ dysfunction syndrome, the condition referred to as sepsis should be also be identified, since many critically ill patients with sepsis develop multiple organ failure. In the past, the term sepsis was commonly used for the clinical response arising from an infection, but the presence of an identical clinical response without the presence of a documented infection has led to the use of confusing terminology, such as septicaemia and non-bacterial sepsis. Nowadays, the term sepsis is exclusively used for patients with a systemic response to a documented infection and; the term septicaemia should be dropped from common usage. 


\section{Systemic inflammatory response syndrome}

Experience in intensive care has shown that a similar, or even identical, response to sepsis can arise in the absence of infection. This syndrome is termed systemic inflammatory response syndrome (SIRS) (5). This response can be seen following a wide variety of insults, such as traumatic shock, and includes, but is not limited to, more than one of the following clinical manifestations indicating an increased inflammatory response: (1) a body temperature exceeding $38.0^{\circ} \mathrm{C}$ or less than

$36.0^{\circ} \mathrm{C}$; (2) a heart rate exceeding 90 beats per minute; (3) an increased breathing rate, exceeding 20 breaths per minute, or hyperventilation, as indicated by a $\mathrm{PaCO}_{2}$ of less than $32 \mathrm{mmHg}$; and (4) a change in the white blood cell count to a value exceeding $12,000 / \mathrm{cu} \mathrm{mm}$, a value below $4,000 / \mathrm{cu} \mathrm{mm}$, or the presence of more than 10 percent immature neutrophills ('bands'). These physiologic changes should present an acute alteration from baseline values in the absence of other known causes for such abnormalities, such as chemotherapy-induced leucopoenia.

\section{Severe sepsis}

Severe sepsis is sepsis associated with organ dysfunction, hypoperfusion abnormalities or sepsis-induced hypotension. The hypoperfusion abnormalities include lactic acidosis, oliguria, and acute alterations of mentall status (5).

\section{Sepsis-induced hypotension/septic shock}

The finding of a systolic blood pressure $90 \mathrm{mmHg}$ or its reduction by $40 \mathrm{mmHg}$ or more from baseline and sepsis (in the absence of other causes of hypotension) defines sepsis-induced hypotension (5). Septic shock is defined as sepsis-induced hypotension persisting despite apparently adequate fluid resuscitation, along with the presence of hypoperfusion abnormalities or organ dysfunction.

The next part of this chapter discusses the background of compensated shock and inflammation in the pathophysiology of organ failure and sepsis, to illustrate why specific monitoring techniques were used in the studies reported on in this thesis.

\section{PATHOGENESIS OF MULTIPLE ORGAN FAILURE, WITH SPECIAL REFERENCE TO HEPATOSPLANCHNIC DYSFUNCTION}

Some of the earliest studies of the development of MOF focused on the presence of an infection. Patients sustaining an infection after trauma (e.g. gangrene in a gunshot wound) had a high mortality due to the development of organ dysfunction. Moreover, 
the clinical symptoms observed during such severe infections were similar to those found in patients with MOF. Fry et al. demonstrated that 98 percent of trauma patients who developed MOF had septic features (6), and concluded that multiple organ failure was the 'fatal expression of an uncontrolled infection'. Therefore, if the patient had no obvious infection, such as a pulmonary infection or an infection at the trauma site, a hidden abdominal infection was supposed to cause this 'infection-like' syndrome. As a consequence, physicians performed repeated laparotomies during the subsequent period to search for occult abdominal abscesses in these patients. However, the frequency of occult abscesses thus detected was disappointingly low. In 1985, two studies challenged this view of the pathophysiology of MOF. Norton et al. demonstrated that adequate drainage of intra-abdominal abscesses had no effect on mortality in 21 MOF patients (7). In the other study, Coris et al. assessed the risk of MOF development in patients with trauma and in a group of patients with intra-abdominal infection (4). If the theory of occult intra-abdominal abscesses was correct, the latter group should have a similar risk of developing MOF. Instead, they found a significantly higher risk of developing multiple organ failure in the patients with known intra-abdominal sepsis. The authors suggested that an autodestructive inflammatory reaction was responsible for the failure of the organs in the trauma patients, rather than an (occult) infection.

It is now recognised that such an inflammatory reaction, clinically defined as SIRS, occurs in a variety of conditions, such as burns, pancreatitis and trauma, as well as in patients with infections, with a clinical presentation that ranges from a relatively benign to a severe condition, characterised by MOF. Current research is focusing more closely on the inflammatory response to traumatic insults, independent of infection. The present thesis, too, mainly focuses on the study of non-infectious pathogenesis of MOF.

The pathogenesis of organ failure is complex. Two distinct patterns have been proposed by which shock and trauma can lead to multiple organ failure. The first is a 'one-hit model', in which massive tissue damage and severe uncontrollable shock combine to evolve directly into MOF (Figure 1). The more common pattern is a 'two-hit model', in which less severe tissue damage and (compensated) shock produce a state of less severe critical illness, defined as SIRS (8). However, these patients become susceptible to the detrimental effects of secondary insults, which may induce a late form of MOF in patients with SIRS. Besides infectious insults that can cause a SIRS-like clinical picture, noninfectious pathologies include burn injury, pancreatitis, ischaemia of an extremity and multiple trauma. Patients with SIRS are prone to developing organ system dysfunction, such as renal failure, shock and multiple organ dysfunction syndrome (MODS) or MOF (9).

Several hypotheses have been formulated to explain which mechanisms are responsible for the development of MOF in the absence of an infection. These include unrecognised tissue hypoxia or dysoxia, microcirculatory failure, gut barrier failure with bacterial translocation, ischaemia/reperfusion injury and activation of the inflammatory cascade (Table 1). These hypotheses are discussed in the next part of this chapter. 


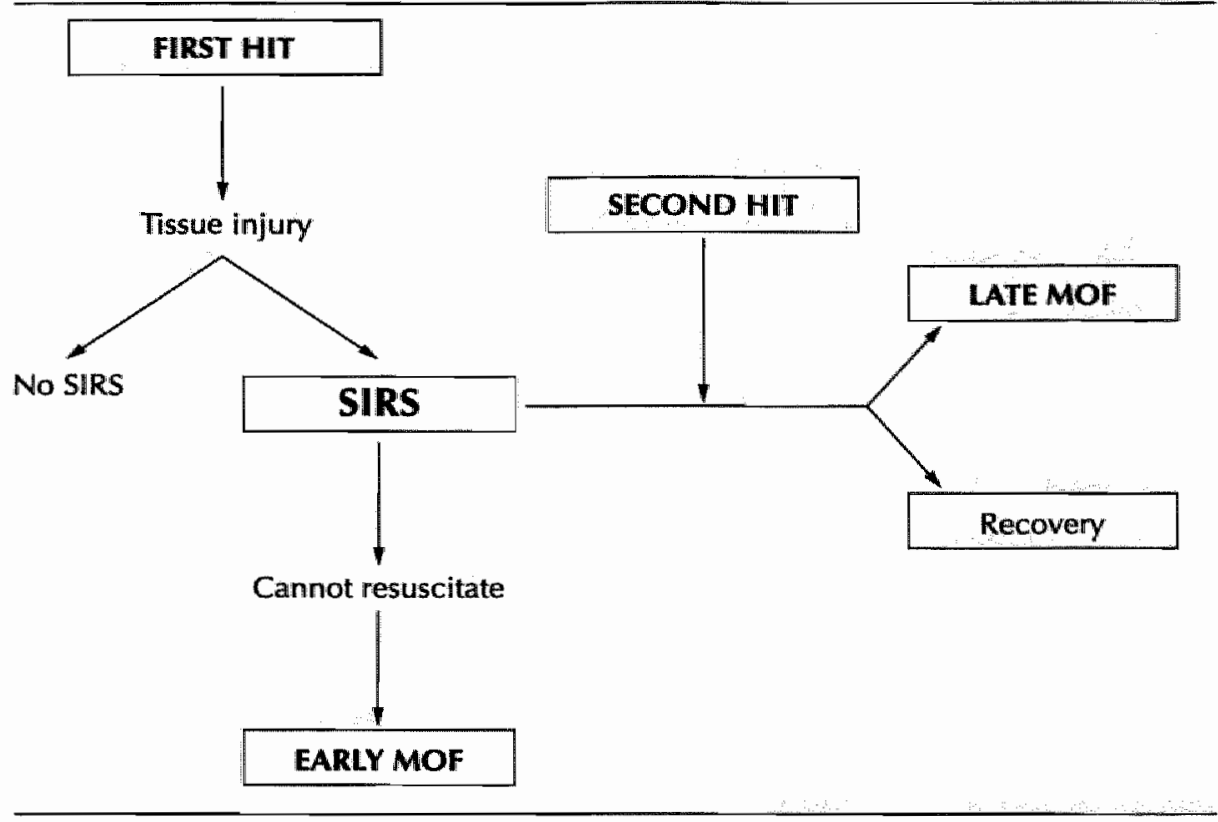

Schematic depiction of the pathophysiology of the development of multiple organ failure (MOF). Some degree of tissue injury occurs in patients subjected to surgery, trauma or other noxious stimulus. Depending on the degree of injury, there may be no systemic inflammatory response, or a systemic inflammatory syndrome may dewelop, described as SIRS (systemic intlammatory response syndrome). The inability of the patient to respond to this syndrome, either due to limited capacity or inadequate intervention, cam lead to the development of early MOF. Patients mol develloping early MOF are at risk of developing secondary or late MOF due to a combination of two factors: individual vulnerability and the occurrence of a second noxious stimulus.

\section{Figure 1}

\section{Table 1 Proposed pathophysiological mechanisms of MOF}

\section{Unrecognised tissue hypoxia/dysoxia}

Microcirculatory failure

Ischaemia-repertusion injury

Neutrophil-endothelial interaction

Capillary plugging, disseminated intravascular coagulation

Hepatosplanchnic dysfunction

Splanchnic hypoperfusion

Gut barrier failure

Intestinal bacterial and endotoxin translocation

Priming and activation of inflammatory cascade

Molecullar mechanisms.

Failure of programmed cell death (apoptosis)

Induction of cellular stress reponse (acute-phase responses)

Genetic predisposition 


\section{Unrecognized flow-dependent oxygen consumption}

Many studies of the pathophysiology of MOF have concentrated on the presence of shock, defined as low blood pressure, which had been identified at an early stage as a marker of increased risk of developing organ failure (10). However, correction of this low blood pressure to normal values did not reduce the mortality in MOF patients $(11,12)$.

The main purpose of the circulation in the human body is the adequate delivery of sufficient amounts of nutrients and oxygen to the tissues and the adequate removal of waste products from the tissues. A new definition of shock was proposed, which defined the condition as a lack of oxygen supply relative to the oxygen requirements of the tissues (13). In patients with sepsis, insufficient oxygen is delivered to the tissues, despite increased oxygen delivery, due to the major increases in axygen demand in the tissues. Under normal conditions, the delivery of oxygen is closely related to the amount of oxygen consumed in the tissues. Oxygen delivery $\left(\mathrm{DO}_{2}\right)$ is calculated as the product of the cardiac output (CO) and arterial oxygen content. Oxygen consumption $\left(\mathrm{VO}_{2}\right)$ is calculated as the product of $\mathrm{CO}$ and the arterial-venous oxygen content difference. In the case of a decrease in $\mathrm{DO}_{2}$ (e.g. in the case of cardiac tamponade or during anaemia), $\mathrm{VO}_{2}$ is maintained at a relatively constant level, since the tissues can increase the oxygen extraction ratio $\left(\mathrm{O}_{2} \mathrm{ER}\right)$, defined as the amount of oxygen which is extracted from the blood (Figure 2). The decrease in $\mathrm{DO}_{2}$ can thus be compensated until a certain, critical level is reached, below which further decrease in $\mathrm{DO}_{2}$ is accompanied by a decrease in $\mathrm{VO}_{2}$ (14). This critical level in anaesthetised humans has been estimated to be $330 \mathrm{ml} / \mathrm{min} / \mathrm{m}^{2}$ or $8-10 \mathrm{ml} / \mathrm{min} / \mathrm{kg}$ (15).

It has been suggested that this relationship between $\mathrm{DO}_{2}$ and $\mathrm{VO}_{2}$ changes considerably during sepsis and inflammation. Several studies have found $\mathrm{VO}_{2}$ to be dependent on $\mathrm{DO}_{2}$ (flow-dependent) even above the normal critical $\mathrm{DO}_{2}$ level (Figure 2) (16).

In a study by Tuchschmidt et al, critical oxygen delivery was $15 \mathrm{ml} / \mathrm{min} / \mathrm{kg}$ in patients with sepsis, which is higher than the normal critical level (17). Apparently, these patients require a higher $\mathrm{DO}_{2}$ to maintain adequate tissue oxygenation. In critically ill patients, oxygen delivery is not adequate to meet the oxygen demand in the tissues (i.e. inadequate oxygen extraction). This disbalance causes tissue hypoxia (also termed 'dysoxia') and has been proposed as one of the mechanisms by which organ cell dysfunction and failure occur. However, there are some objections to this theory of flow-dependent oxygen consumption. Firstly, the mathematical link which exists between $\mathrm{DO}_{2}$ and $\mathrm{VO}_{2}$ has been suggested by some authors to be the only argument for such a relation between $\mathrm{DO}_{2}$ and $\mathrm{VO}_{2}$ $(18,19)$. This criticism has, however, not been confirmed by studies using separate techniques for measuring $\mathrm{DO}_{2}$ and $\mathrm{VO}_{2}$. Secondly, tissue measurements of oxygen levels have not always shown low oxygen concentrations (20). An explanation accounting for this situation in sepsis, with high oxygen delivery and normal or high tissue oxygen levels, is based upon the pathologic redistribution of perfusion which occurs during sepsis. This leads to a condition where occult hypoxaemic 


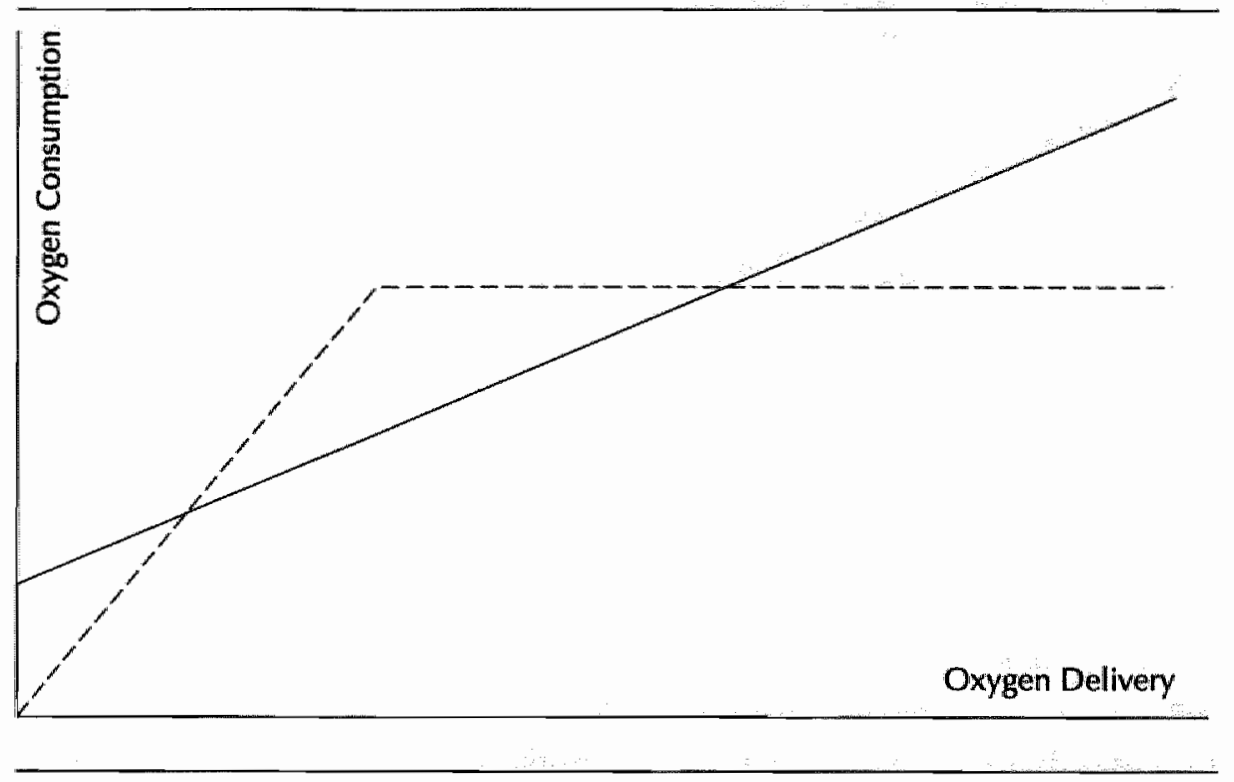

Schematic depiction of the theory of flow-dependent axygen consumption. Under normal conditions (----) lowering the oxygen delivery does not fead to a decrease in oxygen consumption until a certain critical oxygen delivery level is reached, below which there is a direct relationship between decrease in oxygen delivery and oxygen consumption. Under septic conditions ( $\longrightarrow$ ) this critical level of oxygen delivery is reached at a much higher level of oxygen delivery.

\section{Figure 2}

tissue areas can coexist with well-perfused or even overperfused normoxaemic areas. Such a pathologic redistribution by shunting of some parts of the smaller vessels (microcirculation) is thought to be caused by disturbed microvascular regulation through the actions of several inflammatory mediators, such as cytokines and nitric oxide (see below). Experiments studying the microcirculation have indicated four principal mechanisms that could cause pathologic shunting of the microcirculation in organs (21): a) convective arteriovenous shunting through anatomical anastomoses, b) direct diffusion of oxygen from arterioles to venules lying in close proximity to each other, c) altered heterogeneity of the microvascular architecture and d) the inability of haemoglobin to off-load oxygen fast enough to the tissues (21). These mechanisms may explain the presence of relatively dysoxic states despite sufficient oxygen in the circulation.

Another hypothesis to explain why normal tissue oxygen levels and high oxygen delivery levels may be associated with decreasing organ function is the occurrence of cellular dysfunction (22-24). Normally, the principal substances from which cells extract energy are oxygen and one or more of the nutritional components, carbohydrates, lipids and proteins. Intracellularly, these components 
react with the oxygen, reactions that are catalysed by various enzymes. Since almost all these oxidative reactions occur inside the mitochondria, mitochondrial dysfunction prevents oxygen from being processed, thereby causing a lack of oxygen in the metabolic processes, even though sufficient oxygen is available in the mitochondrium. Recent data suggest that both mechanisms may play a role in the continued development of organ dysfunction in critical illness (22), although it remains unclear, which is the most important one.

\section{Ischaemia-reperfusion and neutrophil-endothelium interaction}

During periods of ischaemia of an organ (i.e., an absolute (embolus) or relative (low oxygen delivery) cessation of blood flow to the organ), organ function is impaired by the induction of cell death and tissue damage, depending on the extent and duration of the ischaemic insult. Reperfusion then causes a paradoxical aggravation of the ischaemic injury. Ischaemia-reperfusion injury is thus defined as the tissue injury that occurs after restoration of temporarily interrupted blood supply to an organ or tissue (25). Moreover, experimental studies have shown that ischaemia-reperfusion of various individual organs may also lead to injury of remote organs, despite adequate perfusion of these distant organs (26-30). It has been shown that reperfusion after ischaemia leads to the production of oxygenderived free radicals, predominantly by the endothelium of the microcirculation. This phenomenon also occurs during no-reflow or persistent low-flow states (31). The free radicals formed during reperfusion or no-reflow states are very reactive and unstable molecules, which can target all macromolecules in the body. Carbohydrates become hydrolysed, proteins undergo structural and functional alterations, lipid peroxidation may result in a self-perpetuating mechanism of free-radical production, and oxidation of nucleic acids may result in altered DNA structure (32-34). These oxygen-derived free radicals are involved in a cascade of humoral and cellular events inducing the production of several inflammatory mediators, such as arachidonic acid metabolites, cytokines, activated complement proteins (35). Experimental studies indicated that these oxygen-derived free radicals and activated neutrophils and inflammatory mediators are transported by the circulation from the ischaemic organ to distant organs, causing organ dysfunction downstream from the original ischaemic organ $(29,30)$.

The production of oxygen-derived radicals is an important stimulus for the chemotaxis and activation of neutrophils at the endothelium (36). Leucocytes play a pivotal role in host defence mechanisms and as mediators of tissue destructive events in inflammation. The process of leucocyte-endothelium interaction that underlies the chemotaxis and activation of neutrophils appears to be a multistep cascade, in which each step has to be completed before the next one step can occur. The successive steps are activation, adhesion to the endothelium and transmigration of neutrophils into the target tissue. Eventually, these neutrophils cause injury by producing oxidants and proteolytic enzymes. 
Nitric oxide (see below) is one of the important oxygen radicals, and reacts with another free radical superoxide, to form peroxynitrite, which eventually decomposes into the highly toxic hydroxyl radical. Although much of the initial work studying the ischaemia-reperfusion mechanism was performed in the gut, the mechanism can be applied to numerous organs (e. $g$. heart, kidney, skin). However, the gut is particularly vulnerable to the detrimental effects of ischaemia and reperfusion.

\section{Coagulation abnormalities}

The host response to severe infection involves activation of the immune system, including the rellease of a complex cascade of pro-and anti-inflammatory cytokines. More recently, it has been appreciated that this cytokine response mediates activation of the coagulation system and subsequent inhibition of the fibrinolytic system (37). Ultimately, unregulated activation of the coagulation cascade can progress to disseminated intravascular coagulation (DIC). The DIC syndrome is the most severe clinical manifestation of coagulation abnormalities during sepsis and multiple organ failure, and is characterised by the deposition of fibrin in the milcrovasculature, lleading to a widespread microvascular thrombosis, inadequate tissue perfusion and organ failure. This hypercoagulable state leads to the depletion of clotting factors and platelets and, paradoxically, can result in severe haemorrhage.

However, it is now recognised that this unregulated activation of the coagulation cascade can contribute to organ dysfunction in a much earlier phase of multiple organ failure than was previously thought $(38,39)$. Signs of an activated haemostasis system can already be seen during SIRS $(40,41)$.

Activation of the coagulation cascade can be detected in most patients with sepsis (42). Patients with acute DIC, show variable degrees of thrombocytopenia, hypofibrinogenamia, and prolongation of prothrombin time (PT) and activated partial thromboplastin time (aPTT). Assays for fibrin split products (FSP), fibrin(ogen) degradation products (FDP) or D-dimer fragments of fibrin generally show elevated values. Initially, both PT and aPTT tend to be normal, while platelet count is reduced. By contrast, a consistent elevation of FDP, FSP and D-dimer is found at this stage. In critically ill patients, a blunted increase in the platelet count during their stay at the ICU predicts poor outcome (43). A number of studies have pointed out an important role of AT III and protein $C$ in the process of disseminated coagulation abnormalities during sepsis, since supplementing both agents during sepsis attenuated the features of DIC $(44,45)$. One recent study found a reduced mortality among patients with septic shock using a recombinant activated protein $\mathrm{C}$ treatment (46).

The liver plays an important role in the coagulation cascade. Many of the coagulation factors, such as fibrinogen, prothrombin, and the factors $V$, VII, IX and $X$, as well as inhibitors of both coagulation and fibrinolysis, are synthesised by the liver. The synthesis of coagulation factors is apparently a relatively stable and rapidly 
recoverable function of the hepatocyte. In addition, the increased frequency of coagulation disturbances during disease states such as liver cirrhosis and after liver transplantation is well documented. Dysfunction or failure of the liver during sepsis causes an increased procoagulant state. During sepsis, bacterial products trigger the development of DIC via the tissue factor-dependent pathway of coagulation, resulting in massive thrombin generation and fibrin deposition in the liver. Recent animal experiments have demonstrated that hirudin, a thrombin inhibitor, reduced fibrin deposition in the liver and decreased mortality in endotoxin-induced DIC (47). In a murine sepsis model, the injection of hepatocyte growth factor significantly attenuated the coagulation abnormalities (48). Moreover, AT III, which is synthesised in the liver, is reduced during sepsis. In animal studies, AT III concentrates were found to suppress signs of DIC, prevent MOF and reduce mortality (41). On the other hand, a study by Ruttinger et al. found that blocking Kupffer cell function failed to prevent DIC in septic rats, despite improved survival (49).

\section{Splanchnic perfusion}

The splanchnic organs include the liver, stomach, spleen, pancreas and small and large intestines. The upper gastro-intestinal tract is supplied with blood through the celiac trunk (Figure 3) with the main branches from the celiac trunk supplying the liver (common hepatic artery), stomach (common hepatic, splenic, and left gastric arteries), duodenum (common hepatic artery), spleen (splenic artery) and pancreas (common hepatic and splenic arteries). The lower intestinal tract generally draws its blood supply from two main arteries: the superior and inferior mesenteric arteries. Anatomical variations in this scheme are common, which means that the duodenum and pancreas are usually also supplied by the superior mesenteric artery.

The liver receives blood from both the hepatic artery and the portal vein $( \pm 70 \%)$, which drains blood from all the other splanchnic organs. The venous outflow from the liver runs through the hepatic veins to the inferior vena cava.

Variations in splanchnic macrocirculatory perfusion are known to occur in critically ill patients (50). The splanchnic region participates in the regulation of systemic blood pressure and volume. In patients with shock, perfusion to the vital organs, such as the brain and heart, is maintained to a considerable degree of hypotension. One of the compensatory mechanisms which stabilise blood pressure involves decreasing the blood flow to the splanchnic organs. In patients with acute hypovolaemia, maintaining adequate perfusion of vital organs has been considered of primary importance, while until recently, maintaining adequate perfusion of the splanchnic system during resuscitation used to be considered of no clinical consequence (51). Since the splanchnic organs were considered to be relatively 'dormant' organs, little attention was paid to maintaining adequate splanchnic perfusion in these patients in previous decades.

Recently, however, attention has focused on the effects of regional hypoperfusion, both at the macrocirculatory and microcirculatory level of the splanchnic system, in the critically ill and surgical patients. Numerous studies 


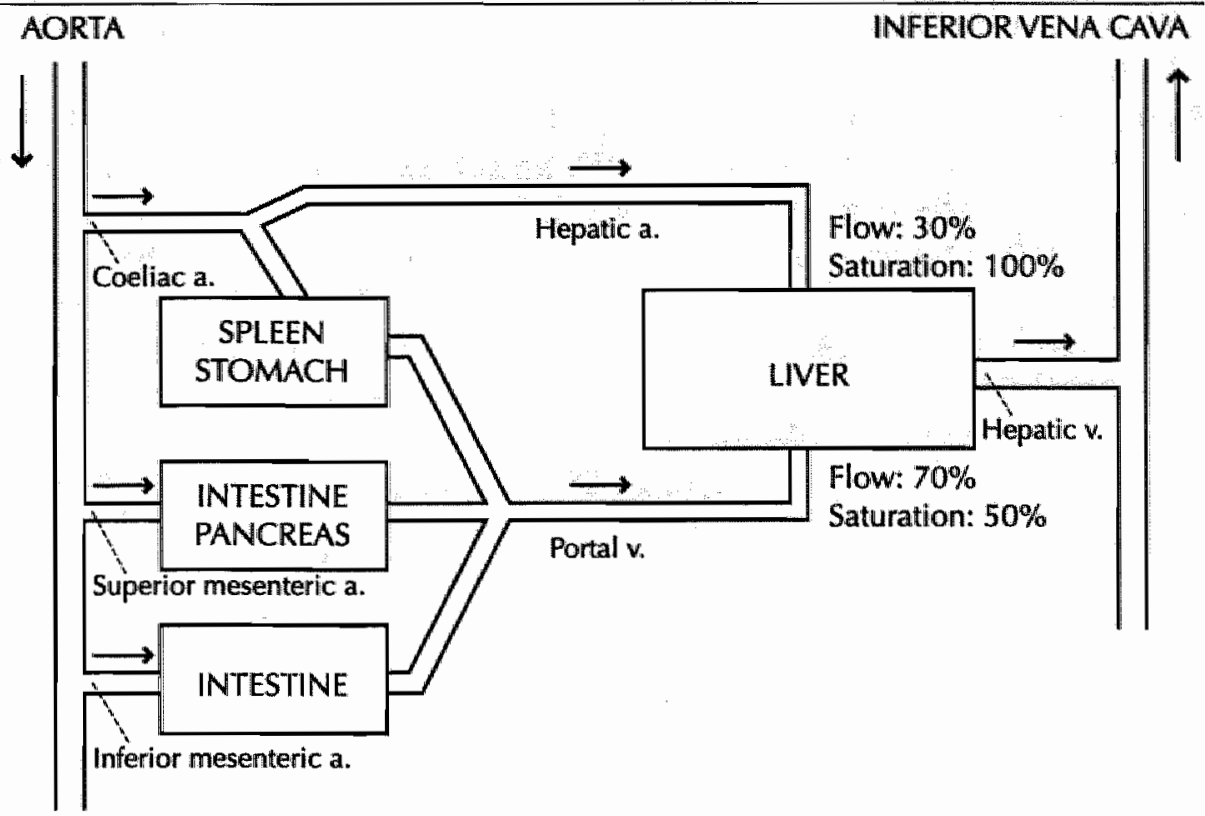

Schernatic depiction of the anatomy of the hepatosplanchnic vasculature. The aorta gives off three mesenteric branches: the coeliac artery and the superior and inferior mesenteric arteries. The liver is supplied by two vessels: the hepatic artery, which supplies $30 \%$ of the btal flow to the liver, providing blood with a high oxygen concentration, and the portal vein, which delivers $70 \%$ of the lotal supply, but at a much lower oxygen concentration. The hepatic vein finally enters the inferior caval wein.

Figure 3

have suggested that the splanchnic region contributes to the development of MOF $(26,52,53)$. Several authors have demonstrated that in low-flow states, such as haemorrhagic shock, splanchnic perfusion decreases out of proportion to the overall decrease in cardiac output. In a study by Dalton et al., healthy volunteers underwent a $10 \%$ haemorrhage, without changes in global vital signs or haematocrit values (54). However, flow in the celiac artery and superior mesenteric artery, as measured with duplex ultrasonography, was significantly decreased. In another study, healthy volunteers underwent simulated hypovolaemia by application of negative pressure on the lower body (55). This hypovolaemia caused a splanchnic vasoconstriction, which remained present after recovery from the simulated hypovolaemia, while other cardiovascular and biological variables returned to normal. Apparently, then, the splanchnic circulation is the last to be restored to normal after resuscitation. Although this phenomenon was observed in healthy volunteers, 
it is increasingly regarded as important in critically ill patients, due to loss of vasomotor control (56).

Failure of the vasomotor control during inflammation has focused research not only on the macrocirculation, but also on intestinal villus perfusion. The villi and intestinal mucosa are supplied by a complex network of vessels, the microcirculation. Blood is normally supplied by a central arteriole and is drained by venules surrounding it. The direction of the blood flow in the arterioles is opposite to that in the venules, creating a counter-flow mechanism (57) (Figure 4), which facilitates the exchange of oxygen from the artery to the vein.

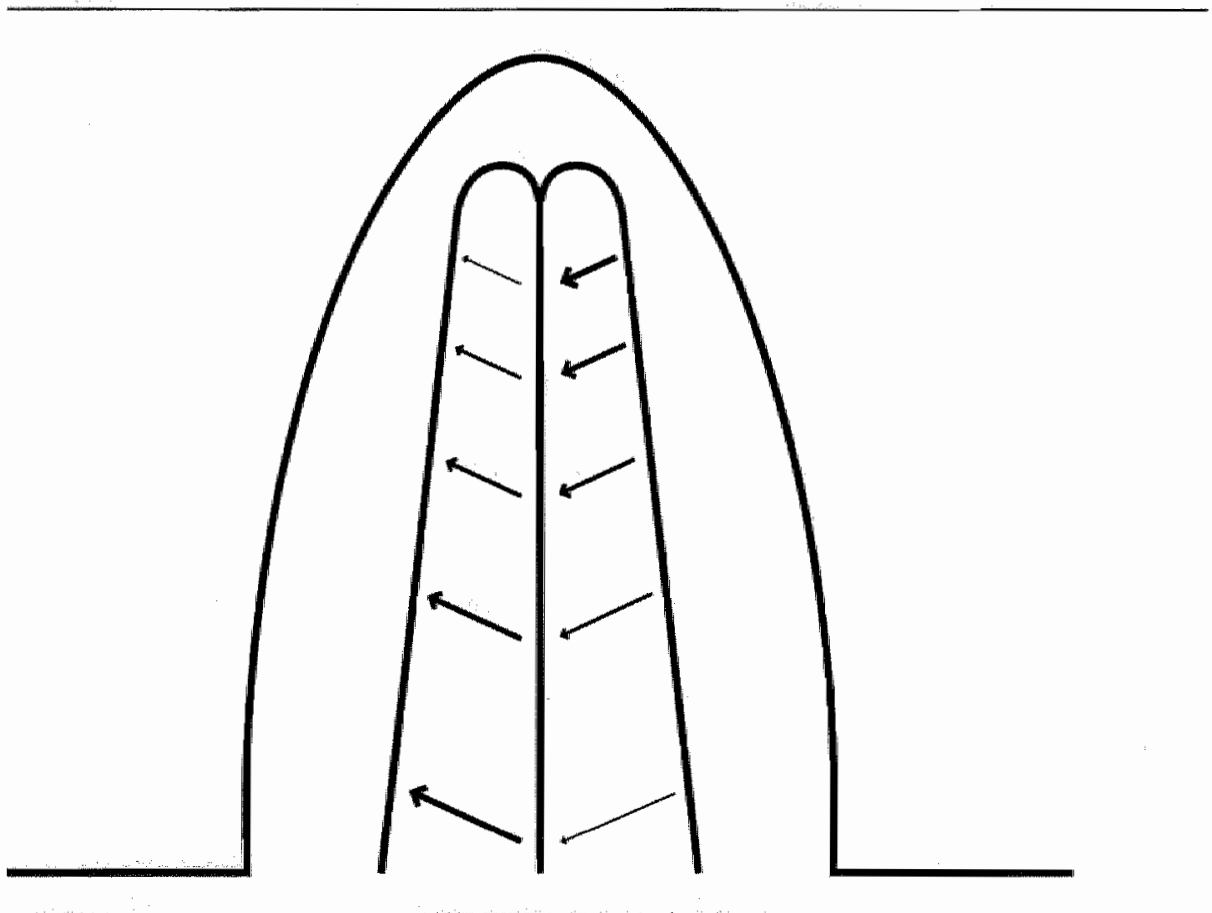

Schematic deptetion of the counter-flow mechanism in the villus of the intestinal mucosa. A central arteriole delivers oxygen rich blood to the villus tip from which venules caury oxygery-poor blood away from the villus. The oxygen-poor blood actively attracts oxygen from the oxygen-rich blood by diffusion, which thereby becomes poorer in oxygen. The apposite process occurs for the $\mathrm{CO}_{2}$ from the top of the villus to the bottom.

Figure 4

However, this countercurrent exchange of oxygen makes the tip of the villus more susceptible to hypoxic or hypoperfusional events, since the oxygen saturation of the arteriolar blood is high at the base of the villus and low at its tip. Moreover; the arterioles that supply the villus branch off at right angles and the presence of pre-capillary sphincters renders the mucosal layer susceptible to absolute or rela- 
tive oxygen shortage during low-flow conditions and septic shock. Humoral (vasopressin and angiotensin $1 \mathrm{I}$, autonomic (neurally mediated -adrenergic stimulation of postcapillary venous beds), and local factors (nitric oxide, cytokines) appear to be involved (58). In addition, external factors such as diet and exogenously administered catecholamines can also influence spllanchnic perfusion (59-61).

During shock or hypovolaemia splanchnic blood flow is decreased, which induces intramucosal acidosis (62). The vasoregulatory control of splanchnic perfusion is preserved during a long period in these conditions. Thus, this decreased flow and the occurrence of acidosis is due to splanchnic vasoconstriction. Bitterman et al. showed that superior mesenteric artery blood flow could be increased with hypertonic saline after haemorrhagic shock (63). By contrast, Oud et al. showed that prompt and adequate restoration of the systemic blood pressure after resuscitation failed to restore gastric intramucosal pHi, which is a measure of gut mucosal oxygenation (see chapter 2) (64). This prolonged intramucosal acidosis and mucosal hypoperfusion (65) increases the risk of splanchnic hypoxia, which implies an increased risk of developing a persistent inflammatory response (66) and subsequent organ damage.

A number of studies using modulation of splanchnic perfusion with vasoactive agents have tried to increase our understanding of the behaviour of the splanchnic circulation during septic and non-septic conditions. Johnson et al. showed that portal flow is restored by dopamine after a significant reduction in flow by non-bacterial acute lung injury and the use of positive end-exspiratoy pressure ventilation (67). In dogs, the use of dopamine after cardiopulmonary bypass increased gastric blood flow, but not intestinal blood flow (68). In contrast, dobutamine was able to elevate the blood flow in most visceral organs in this study. In a study by Chintala et all, dopexamine restored splanchnic perfusion after haemorrhage to $80-100 \%$ of baseline level (69). Unfortunately, this study did not measure intramucosal $\mathrm{pHi}$, so it provides no data about the effects of dopexamine on mucosal oxygenation in experimental non-endotoxaemic models.

In addition to the experimental studies, numerous clinical studies have modulated splanchnic perfusion with vasoactive substances during hypovolaemia or non-septic (low-flow) conditions. Gastric intramucosal pHi was found to decrease significantly in response to major abdominal surgery in patients who developed complications (70) and this correlated well with systemic levels of inflammatory mediators in the blood. Such a decreased pHi has also been seen in patients undergoing orthotopic liver transplantation or cardiopulmonary bypass, and in patients with acute circulatory failure and small volume haemorrhage $(54,71-74)$. Just as in experimental models, reversal of systemic perfusion abnormalities after transient hypovolaemia led to a protracted reversal of gastric pHi (55). Since vasoregulation remains functional in hypovolaemia, occult systemic hypovolaemia after resuscitation is compensated over a prolonged period by increased splanchnic vasoconstriction and reduced splanchnic blood flow. Restoring this occult hypovolaemia, especially in peri-operative patients, by administering fluids has been shown to improve splanchnic perfusion and outcome (75). However, prolonged periods of splanchnic hypoperfusion eventually lead to vascular 
dysfunction, tissue damage and organ failure, especially when hypovolaemia is combined with tissue injury, as is the case in patients with sepsis and multiple organ failure. The use of vasopressors may be only partially useful in these patients. In a study by Ruokonen, dobutamine was able to restore splanchnic oxygenation and improve global oxygenation in patients with low cardiac output syndrome after cardiac surgery (76) (Table 2). This effect of dobutamine has been confirmed in patients undergoing liver resection (77). A study of liver transplant patients found that dopamine, dobutamine, and dopexamine were all able to increase total hepatosplanchnic flow and oxygen supply (78).

Table 2

\begin{tabular}{|c|c|c|}
\hline Vasoactive drug & Sepsis & Non-sepsis \\
\hline \multirow[t]{2}{*}{ Dopamine } & TSFat & TSF \\
\hline & $\mathrm{pHi}=+$ & \\
\hline \multirow[t]{2}{*}{ Dobutarinine } & TSF 1 & TSF 1 \\
\hline & $\mathrm{pHi} \uparrow$ & \\
\hline \multirow[t]{2}{*}{ Dopexamine } & $\operatorname{TSF} \uparrow$ & TSF 4 \\
\hline & $\mathrm{pH} H \mathrm{i}=+$ & $\mathrm{pHi}=\downarrow$ \\
\hline Norepinephrine & $\mathrm{TSF} \uparrow$ & $?$ \\
\hline Epinephrine & $\mathrm{pHi} \downarrow$ & $?$ \\
\hline
\end{tabular}

aTSF total splanchnic blood llow; pHi intramucosal pH

The effects of norepinephrine and epinephrine on splanchnic perfusion have not been studied at present in patients with non-septic conditions.

Under baseline conditions, an increase in total hepatosplanchnic blood flow is observed during experimental sepsis. In such situations, however, the metabolic demand increases even more than the flow, resulting in inadequate tissue oxygenation. This inadequate tissue perfusion is complicated by an impaired microvascular function in sepsis, leading to impaired flow regulation in the gut (79) and causing inhomogeneous flow distribution of the microcirculation (80).

The findings on splanchnic perfusion, however, are highly species-dependent. A hyperdynamic normotensive model in the pig showed a decrease in mesenteric blood flow (81), whereas in rats and rabbits, hepatosplanchnic blood flow increased in response to sepsis (81). However, if the challenge is massive and acute, mesenteric blood flow can also decrease in these models. Turnbull found that sepsis decreased blood flow in the superior mesenteric artery temporarily, but that this decrease was sustained if the septic shock had been preceded by haemorrhagic shock (82). The adequacy of resuscitation and the magnitude of the challenge seem to determine the mesenteric blood flow in animal sepsis. 
Fink et al. showed that the decreased mesenteric blood flow in porcine sepsis was accompanied by intestinal mucosal acidosis (83). Restoration of the mesenteric perfusion could be achieved by treating the animals with fluids and dobutamine. but there was no complete recovery of mucosal acidosils. They concluded that this had to be due to the disturbed local microvascular flow regulation induced by endotoxin. This has been confirmed in other studies using dopexamine and dopamine $(79,84)$.

Unlike what was found in the studies by Fink et al., patients with sepsis show higher than normal splanchnic blood flow (85). However, this study found that the increase in oxygen consumption was not matched by an increase in flow, causing a mismatch between oxygen delivery and oxygen consumption in the hepatosplanchnic area despite increased oxygen extraction $(85,86)$. The increased demand in patients with sepsis, accompanied by increased metabolism, seems to be a special hallmark of the changes in perfusion. Correction of hypotension or hypovolaemia in these patients can increase the splanchnic blood flow, indicating that fluid therapy in patients with sepsis can increase perfusion to a level closer to the oxygen consumption requirements (87). However, the mismatch between oxygen delivery and consumption appears to be sustained despite increased fluid or vasopressor therapy.

Patients with sepsis often have significantly decreased gastric mucosal pHi compared to critically ill patients without sepsis (81). The finding of a lower $\mathrm{pHi}$ in patients with sepsis predicts poor outcome (88).

The relation between total splanchnic blood flow and gastric intramucosal hypoperfusion is not yet clear. Dopamine has been found to increase total splanchnic blood flow in septic patients, but to decrease pHi (79) (Table 2). Olson et al. also reported that dopamine was unable to increase intramucosal pHi (89). Norepinephrine had variable effects on total hepatosplanchnic blood flow, but was able to increase pHi (90). However, Ruokonen was unable to find increased splanchnic flow in patients with sepsis treated with norepinephrine, as measured by ICG dilution (91). In a study by Levy et al., gastric mucosal pHi was restored by a combination of norepinephrine and dobutamine, whereas epinephrine decreased

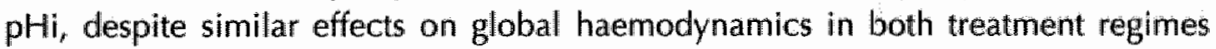
(92). In a study by Gutierrez et al., dobutamine reversed intramucosal acidosis in septic patients (93). It can be concluded from these studies that the various vasoactive agents have different effects on splanchnic perfusion in septic patients. The effects on mucosal blood flow are difficult to predict in individual patients and may differ from the effects on total hepatosplanchnic blood flow (94).

Inadequate perfusion of the splanchnic area seems to be associated with a deterioration in distant organ function, although the pathophysiologic mechanism behind this association is poorly understood (79). Regional flow-dependent oxygen consumption and ischaemia-reperfusion injury have been found to be important contributors to organ dysfunction in the splanchnic region. Some other mechanisms described below are also considered to explain, at least partially, the observed link between splanchnic ischaemia and complications. It must be remembered that these mechanisms may in themselves also be responsible for decreased splanchnic perfusion. 


\section{Altered gut permeability}

One of the earliest observations during inadequate splanchnic perfusion is an increase in permeability to large molecules. The splanchnic region is the largest lymphoid organ in the body and is considered to have an important role to play in a patient's immune defence against infection and inflammation (95). The immunological barrier of the gut is formed by the gastrointestinal lymphocytic system. The distinction between the development of a systemic inflammatory response or the induction of tolerance against an antigen originates from this system, the gut-associated lymphoid tissue (GALT) (96). Experimental data suggest that increased permeability allows viruses and bacteria to enter the intestinal mucosa, via overlying lymphoild follicles, named Peyer's patches, where the mucosal immune response is started. This initiation consists of the uptake and processing of the virus particles or bacteria by macrophages and their presentation to $T$ and $B$ lymphocytes. Macrophage dysfunction has been associated with increased bacterial translocation (96), and the application of G-CSF, which can stimulate macrophage function, did indeed decrease bacterial translocation (97). Primed lymphocytes leave the mucosa and enter the mesenteric lymph nodes, where they proliferate. Subsequently, lymphocytes enter the circulation via the thoracic duct or migrate back to the intestinal mucosa, where their function is effected.

This increase in permeability is thought to result from loss of integrity of the intercellular tight junctions, and the cellular membranes may be damaged after prolonged periods of intestinal ischaemia. Severe or prolonged periods of ischaemia even cause shedding of epithelial cells, which initially involves only the villus tip, but may develop into full mucosal necrosis or even transmural necrosis. Such development of full transmural necrosis is rare during critical illness, but intestinal ischaemia after aortic aneurysm surgery or mesenteric thrombosis often develops into the final stage of intestinal wall necrosis and rupture.

\section{Gut bacterial translocation}

The occurrence of bacterial translacation from the gut may play a role in the relation between splanchnic ischaemia and organ dysfunction in distant organs. The colon is seen as a reservoir from which bacteria and bacterial products (e.g. endotoxin) migrate across the intestinal mucosa (98). It has been shown that bacteria can migrate across the mucosa paracellularly and transcellularly $(99,100)$. When bacteria pass through the lamina propria, they can be cleared by macrophages and transported to the mesenteric lymph nodes. From there, invasion of both the systemic circulation and the lymphatic system has been described. A study by Lemaire et al. showed that products from translocated bacteria were transported through the thoracic duct (101). Other studies have indicated that bacteria and bacterial products are transported to the systemic circulation through the portal vein $(102,103)$. Experimental studies have also shown that these bacteria and bacterial products are transported to the target organs, thereby inducing an 
inflammatory reaction and organ dysfunction. However, whether these routes play a clinically important role in the development of MOF or are just indicators of critical illness is still being debated $(102,104)$. The use of selective decontamination of the gut, as a means of eliminating possible pathogenic organisms from the gut lumen, has so far not been successful in reducing the number of patients with multiple organ failure, despite consistently reducing the number of pathogenic organisms $(105,106)$.

An important preceding phenomenon of bacterial translocation is colonisation of the digestive tract by potentially pathogenic organisms (107-109). Although abnormal colonisation of the gut in critically ill patients may increase the likelihood of translocation from the gut, it is difficult to prove clinically whether the translocated bacteria can eventually cause a systemic infection (110), possibly because of elimination of the translocated bacteria by the liver (111). The elimination of bacteria seems to result in two apparently opposing effects: elimination of toxic products from the circulation and induction of the release of inflammatory mediators into the systemic circulation, produced by bacteria-activated Kupffer cells in the liver, which may damage organs.

Recent studies have increasingly focused on the presence of such a systemic inflammatory response. Bacterially induced or ischemia-reperfusion induced release of mediators (112) may play a more important role in the systemic inflammation seen in patients with SIRS and MOF than the translocation of bacteria itself (113).

\section{Metabolism in the hepatosplanchnic area during sepsis}

\section{Inflammatory response}

One of the hallmarks of critical illness is an increased generalised, systemic inflammatory response. Activation of immune effector cell populations, such as mononuclear phagocytes, which include liver Kupffer cells and peritoneal and intestinal macrophages, represent one of the earliest cellular responses to a stimulus. One of the most extensively investigated stimuli is mononuclear phagocyte activation by endotoxin or lipopolysaccharide, the immunologically active component of gram-negative bacteria. While endotoxin is considered to be the first initiator of the inflammatory response during sepsis and SIRS, a recent study by Ayala et al. suggests that it is not the endotoxin alone, but the devitalisation of tissues during the period of hypoperfusion or ischemia-reperfusion, combined with the effect of endotoxin, which induces the exaggerated inflammatory response to subsequent stimuli (114).

Communication between cells which are involved in the inflammatory reactions depends on cytokines, which are low molecular weight proteins (115). The term cytokines is used to indicate several distinct groups of inflammatory mediators, such as chemokines, tumour necrosis factor (TNF), interleukins (e.g. IL-6, IL-8) and interferons, which regulate the inflammatory response both in a pro-inflammatory and an anti-inflammatory direction. 
The release of TNF in tissues and in the circulation has a variety of effects. TNF has a beneficial role in the defence against infections. At lower concentrations, locally produced TNF may be essential in the maintenance of a well-contained, physiologic inflammatory response, inducing both autocrine and paracrine effects on the surrounding cells (116). However, TNF is also undoubtedly involved in the haemodynamic and metabolic derangements seen in endotoxaemia (117). Infusion of TNF in animals or humans has been found to induce sepsis-like symptoms, depending on the dosage used $(118,119)$. At higher concentrations, TNF induces local tissue injury. Systemically, the release of circulating TNF causes immune cell activation and a significant alteration of the patient's organ function (120). TNF is considered to be one of the most important inflammatory mediators in causing, hypotension, depression of the cardiac function and vascular leakage.

The release of $1 \mathrm{~L}-6$ modulates the extent of fever, tachycardia, catabolism and leukocytes during sepsis $(121,122)$. IL-6 is released by mononuclear macrophages, vascular endothelial cells and fibroblasts in response to IL-1 and, to a lesser extent, TNF (123). During endotoxin infusion, IL-6 is secreted in response to TNF or IL-1 rather than to endotoxin itself. IL-6, also called the hepatocellular stimulatory factor, causes hepatocytes to synthesise plasma proteins, such as fibrinogen, that contribute to the acute phase response. Injection of IL-6 does not result in the haemodynamic deterioration seen after injection of TNF or LPS. While infusion of anti-IL-6 antibodies abrogates the effects of TNF, higher doses of TNF override this attenuation (124).

IL-8 is produced by mononuclear phagocytes, polymorphonuclear leucocytes, endothelial cells, epithelial cells and a variety of other cells in response to stimuli like endotoxin and TNF. The primary function of IL-8 is to activate and attract neutrophils to sites of inflammation. In addition to this function, IL- 8 has been implicated in angiogenesis and the process of attracting T-lymphocytes and basophils (125).

Specific membrane-bound receptors regulate the biological activity of cytokines and other inflammatory mediators. In addition, soluble cytokine receptors can compete with the membrane-bound receptors and thereby modullate the cytokine-induced signalling (e.g. soluble TNF receptor (s-TNF-R)-55 and -75). TNF receptors are present on practically all cells analysed and come in two forms, with molecular weilghts of $55 \mathrm{kD}$ and $75 \mathrm{kD}$, respectively. The soluble forms of these receptors result from proteolytic cleavage of the extracellular part of the TNF celli surface receptor from activated target cells $(120,126)$. The function of the s-TNF-Rs has not yet been fully elucidated (127). The shedding of TNF-Rs seems to function as a protective mechanism against excessive harmful effects of TNF. Soluble TNF receptors are released in response to the production of TNF. This can affect TNF activity by competing with the cell-associated TNF receptors, thereby interfering with its binding to and activation of cells. On the other hand, binding of TNF to the s-TNF-Rs stabilises the structure and preserves the activity of TNF, thus augmenting its effects (128). Hence, s-TNF-Rs are thought to be involved in the control of cytokine response during inflammation and sepsis $(129,130)$.

Other inflammatory mediators are involved in neutrophil adhesion to the vessel wall and migration into reperfused tissue. Three families of adhesion receptor 
molecules expressed on leucocytes and endothelial cells have been defined: the integrin, immuno-globulin related and selectin molecules. Integrins mediate cell-to-cell and cell-to-matrix interactions and transduce signals into the cell. Selectins (e.g. E-selectin) mediate relatively non-adherent contacts between leucocytes and endothelial cells, resulting in leucocytes 'rolling' along the endothelium. Whereas E-selectin has not been detected on non-stimulated vascular endothelium, soluble E-selectin levels have been detected in sera of normal individuals. It has been suggested that the soluble form is constitutively synthesised by the vasculature. Although little is known about its function, elevated s-E-selectin levels might be an indicator of increased endothelial activation, as in inflammation. The presence of $\mathrm{s}$-E-selectin in vivo may inhibit leukocyte adhesion to E-selectin expressing endothelial cells. In contrast, interaction between leucocytes and s-E-selectin causes neutrophils to express enhanced adhesive activity. These can in turn bind to the adhesion molecules of endothelial cells; such as ICAM (intracellular adhesion molecule)-1, facilitating influx of neutrophils. Subsequent interaction between leucocyte integrins and ICAM-1 on activated endothelial cells consolidates this binding and promotes spreading and locomotion of adhering cells $(131,132)$. This expression is normally low, but the release of cytokines, such as TNF and IL-1, at sites of inflammation causes cell activation and results in augmented cellular expression of ICAM-1. The level of ICAM-1 expression reaches a stable plateau after 16 to $24 \mathrm{hrs}$ (133).

The hepatosplanchnic area plays an important role in the inflammatory response during multiple organ failure. Various noxious stimuli to the gut can result in the development of multiple organ failure, mediated in part by activation of the cytokine cascade in the gut and the liver. A number of experimental studies have indicated that the enterocytes of the gut can produce inflammatory mediators in response to endotoxin and thermal injury $(134,135)$. Chaudry (6) and Ayala (3) showed that haemorrhagic shock caused enhanced systemic release of TNF. Interestingly, this occurred independently of the release of endotoxin in the circulation $(136,137)$. Subsequently, Tamion and co-workers showed that intestinal ischaemia and reperfusion induced intestinal synthesis of TNF and IL-6 which was detectable in the mesenteric blood, while endotoxin was undetectable (138). This study, conducted with rats, found that endotoxin infusion induced similar levels of TNF and IL-6 in the mesenteric blood. In a study by Deitch et al., TNF and IL-6 levels in the portal blood were increased after haemorrhagic shock in rats (1139). Furthermore, alterations in gut function, such as changes in intestinal flora and malnourishment, lead to elevated release of inflammatory mediators by the gut into the circulation (140). Apparently, injuries to the gut by haemorrhage, ischaemia-reperfusion or endotoxin result in the synthesis of inflammatory mediators in the intestine. It is hypothesised that cytokines primarily act in a paracrine fashion to govern metabolic responses to injury, or may exert effects in distant organs when released into the circulation.

In addition to experimental studies, the release of cytokines has also been found in humans. A study by Cabie et al. found all patients undergoing abdominal aortic surgery to have detectable TNF levels, which were significantly higher in the portal 
than the systemic blood (141). By contrast, endotoxin was detected in the portal circulation in $36 \%$ of patients after clamping of the aorta and in $71 \%$ of patients after release of the aortic clamp. This indicates that intestinal ischaemia-reperfusion is a major trigger for cytokine production in humans, not necessarily dependent on endotoxin translocation. In a study by Maeba et al., portal blood levels of IL-6 were increased 6-12 hrs after commencement of abdominal surgery, whereas C-reactive protein levels were at a maximum after 2 days (142). Other studies have identified a central role of Kupffer cells $\left(K C^{\prime} s\right)$, which comprise the major part of the lymphoid tissue in the liver, in the amplification of cytokine production following intestinal production of inflammatory mediators. Towfigh et al. provided proof of the existence of such a gut-liver axis. An in vitro study found increased cytokine production by $\mathrm{KC}^{\prime}$ s after stimulation by the culture medium of intestinal cells exposed to hypoxia (143). Intestinal ischaemia thus directly affected $\mathrm{KC}^{\prime} \mathrm{s}_{\text {, causing }}$ a release of TNF and IL-6. Other studies confirmed the capability of $K C^{\prime}$ 's to release inflammatory mediators. Intraportal infusion of endotoxin in dogs induced a release of TNF by the liver but not by nonhepatic splanchnic organs (144). Koo et al. showed that reduction of the number of $\mathrm{KC}^{\prime}$ 's prior to coecal ligation and puncture (CLP) reduced the release of TNF and IL-6 (145), In a study by Ayala et al., the release of IL-6 after CLP was shown to be unrelated to the sensitivity of the animal to endotoxin (146). In a subsequent study, this group showed that the release of cytokines by KC's induced effects on more distant organs, such as the spleen and the lungs (147). In humans, Douzinas et al. were able to show that patients with established multiple organ failure had an increased release of TNF and IL-6 from the splanchnic area (148). This was confirmed in a more experimental setting in healthy subjects undergoing bolus endotoxin infusion. Hepatic venous levels of TNF were consistently higher than arterial levels (149).

In summary, after a variety of stimuli, both related and unrelated to endotoxin, the gut and the liver are stimulated to release inflammatory mediators, primarily for local control of the noxious stimuli. In the case of an overwhelming stimulus, mediators may overflow from the tissues into the bloodstream, to other organs. This stimulation of the gut-liver axis seem to play an important role in the development of multiple organ failure.

\section{Nitric oxide and arginine metabolism}

Recent developments in the treatment of septic shock indicate an important role of nitric oxide (NO) (150). NO is produced from L-arginine by nitric oxide synthase (NOS). In this reaction, molecular oxygen is incorporated into NO and citrulline. This $\mathrm{NO}$ is an unstable intermediate product and is further oxidised to nitrite and nitrate.

There are three isoforms of NOS: neuronal NOS (NOS1), endothelial NOS (NOS3) and inducible NOS (NOS2) (151). NOS1 and NOS3 are often grouped together as constitutional NOS, as both are expressed constitutively. The expression of NOS1 +3 leads to the production of small amounts of NO. As its name implies, NOS2 is induced by various microbes, microbial products and inflammatory mediators. The amount of NO produced de novo after expression of NOS2 is many times the amount produced after expression of $\mathrm{NOS} 1+3$ (151). 
NO has a dual role. Produced in physiological quantities, it is a crucial mediator involved in proper organ functioning. In the gastrointestinal tract, NO participates in the modulation of the smooth musculature tone, such as the regulation of intestinal peristaltism, gastric emptying and antral motor activity. It is also involved in the maintenance of mucosal blood flow. NO produced by the endothelial form of NOS (NOS3) regulates vascular tone and blood pressure, as well as organ flow distribution. In physiological conditions, $\mathrm{NO}$ acts as an endogenous mediator modulating the repair and integrity of gastro-intestinal tissues, and exhibits gastroprotective properties against various types of noxious stimuli. Enhanced formation of NO by the inducible form of NO synthase (NOS2) contributes significantly to the clinical features of sepsis, such as hypotension and increased vascular permeability. Increased levels of NO seem to be responsible for GI mucosal damage, partly induced by changes in gastric mucosal blood flow (152).

Recently, the hypothesis that induction of NOS1+3 is always beneficial and that of NOS2 is always deleterious has been put into question. Inhibiting enhanced formation of NO by inhibiting NOS, can treat hypotension in septic shock (153), but is also frequently associated with detrimental effects (154), culminating in the reported increased mortality rate in a clinical sepsis study using $L-N^{G}$-methylargininehydrocholine, a non-selective NOS inhibitor (155). Substantial experimental evidence indicates that non-selective inhibition of NOS may lead to increased damage to the hepatic and splanchnic organs $(156,157)$. Inhibition of NOS $1+3$ may therefore be responsible for the persistent and non-correctable splanchnic ischaemia frequently associated with septic shock $(158,157)$. It was expected that selective inhibition of NOS2 would be therapeutically useful in sepsis, by both reducing the endotoxin-induced systemic shock and beneficially influencing hepatosplanchnic perfusion and function. However, studies using selective NOS2 inhibitors have produced conflicting results on the effects of hepatosplanchnic perfusion (157,159-163). Saetre et al. found improved liver circulation and oxygen metabolism during porcine endotoxaemia when using amino-isothiourea (AE-ITU), a selective NOS2 inhibitor (162), On the other hand, Wray et al. did not find reduced organ injury using a selective NOS2 inhibitor ${ }_{f}$ despite the prevention of systemic circulatory failure (160).

Moreover, the supplementation of precursors of $\mathrm{NO}$, such as L-arginine, did not induce uniformly positive or negative effects. In a study by Bruins et al., the infusion of L-arginine during porcine endotoxaemia increased NO synthesis in the portal-drained viscera and liver (164), as measured by stable isotope infusion of L(guadino- ${ }^{15} \mathrm{H}_{2}$ )-arginine and $\mathrm{L}$-(Ureido- $\left.{ }^{13} \mathrm{C}^{-2} \mathrm{H}_{2}\right)$-citrulline. However, no differences in splanchnic plasma flow were found. In contrast, Angele et al. found improved systemic and regional perfusion after supplementing arginine during sepsis (165). Gianotti et al. found that an arginine supplement improved survival in gut-derived sepsis and peritonitis by modulating bacterial clearance (166). 


\section{REFERENCES}

1. Hunter AR. Old unhappy far off things. Some reflections on the significance of the early work on shock. Ann Coll Surg Engl 1967;40(5):289-305.

2. Moore FD. Shock and sepsis: some historical perspectives. Surg Clin North Am 1969;49(3):481-7.

3. Vincent H-L, Thijs $\mathbb{L}$, Cerny V. Critical care in Europe. Crit Care Med 1997;13(2):245-54.

4. Goris RJ, te Boekhorst TP, Nuytinck JK, Gimbrere JS. Multiple-organ failure. Ceneralized autodestructive inflammation? Arch Surg 1985;120(10):1109-15.

5. Bone RC, Balk R.A., Cerra FB, et al. Definitions for sepsis and organ failure and guidelines for the use of innovative therapies in sepsis. Chest 1992;101(6):1644-55.

6. Fry DE, Pearlstein L, Fulton RL, Polk HCjr. Multiple system organ failure. The role of uncontrolled infection. Arch Surg 1980;115(2):136-40.

7. Norton LW. Does drainage of intraabdominal pus reverse multiple organ failure? Am I Surg 1985;149:347-50.

8. Moore FA, Moore EE. Evolving concepts in the pathogenesis of postinjury multiple organ failure. Surg Clin North Am 1995;75(2):257-77.

9. Poeze $M$, Rapan $\downarrow$, Greve JWM, et al. Incidence of systemic inflammatory response syndrome and argan fallure on a mixed intensive care unit - the definitions evaluated. [Abstract] Br I Surg $1997 ; 84:(6) 880-1$.

10. Blow $O$, Magliore $L$, Claridge IA, Butler $K$, Young JS. The golden hour and the silver day: detection and correction of occult hypoperfusion within 24 hours improves outcome from major trauma. J Trauma 1999;47(5):964-9.

11. Deitch EA, Goodman ER. Prevention of multiple organ failure. Surg Clin North Am 1999;79(6): 1471-88.

12. Bishop MH, Shoemaker WC, Appel PL,et al. Relationship between supranormal circulatory values, time delays, and outcome in severely traumatized patients. Crit Care Med 1993;21(1):56-63.

13. Radomski MW, Palmer RM, Moncada S. Glucocorticoids inhibit the expression of an inducible, but not the constitutive, nittric oxide synthase in vascular endothelial cells. Proc Natl Acad Sci USA 1990:87:10043-7.

14. Komatsu T, Shibutani $K$, Kubal K, Kumar K, Sanchala V, Lees DE. Critical level of oxygen delivery after cardiopulmonary bypass. Crit Care Med 1987;15(3):194-7.

15. Shibutani K, Komatsu T, Kubal K, Sanchala V, Kumar V, Bizzarri DV. Critical level of oxygen delivery in anesthetized man. Crit Care Med 1983;11(8):640-3.

16. Dasta JF, Brackett CC. Defining and achieving optimum therapeutic goals in critically ill patients. Pharmacotherapy 1994;14(6):678-88.

17. Tuchschmidt J, Fried J, Swinney $\mathbb{R}_{d}$ Sharma OP. Early hemodynamic correlates of survival in patients with septic shock. Crit Care Med 1989;17(8):719-23.

18. Ronco Jl, Fenwick JC, Tweeddale MG. Does increasing oxygen delivery improve outcome in the critically ill? No. Crit Care Clinics 1996:12(3):645-59.

19. Cain SM. Oxygen supply dependency in the critically illl-a continuing conundrum. Adv Exp Med Biol 1992;317:35-45.

20. Anonymous. Third European Consensus Conference in Intensive Care Medicine. Tissue hypoxia. How to detect, how to correct, how to prevent? Am I Respir Crit Care Med 1996;154(5):1573-8.

21. Ince $C$, Sinaasappel M. Microcinculatory oxygenation and shunting in sepsis and shock. Crit Care Med 1999;27(7):1369-77. 
22. Cairns CB, Moore FA, Haenel $\mathrm{B}$, et al. Evidence for early supply independent mitochondrial dysfunction in patients developing multiple organ failure after trauma. ITrauma 1997;42(3):532-6.

23. Celler ER, Jankauskas $S_{n}$ Kirkpatrick J. Mitochondrial death in sepsis: a defined concept: I Surg Res 1986;40:514-7.

24. Mela-Riker L., Tavakoli H.. Mitochondrial function in shock. Am J Emerg Med 1982;2(1):2-7.

25. Grace PA. Ischaemia-reperfusion injury. Bir / Surg 1994;81:637-47.

26. Fiddian Green RG. Associations between intramucosal acidosis in the gut and organ failure. Crit Care Med 1993;21(2):S103-S107

27. Fontes B, Moore FA, Moore EE, Koike K, Kim F, Trew CE, Peterson VM. Gut ischemia induces bone marrow faillure and increases risk of infection. I Surg Res. 1994,57(4):505-9.

28. Goldman $\mathrm{C}_{\text {, Welbourn }} \mathrm{R}$, Klausner $\mathrm{M}$, Hechtman $H B$. Mast cell and leukotrienes mediate neutrophil sequestration and lung edema after remote ischemia in rodents. Surgery 1992;112: 578-86.

29. Koike K, Moore FA, Moore EE, Poggetti RS, Tuder RM, Banerjee A. Endotoxin after gut ischemia/ reperfusion causes irreversible lung injury. I Surg Res 1992;52(6):656-62.

30. Koike K, Moore FA, Moore EE, Read RA, Carl VS, Banerjee A. Gut ischemia mediates lung injury by a xanthime oxidase- dependent neutrophil mechanism. I Surg Res 1993;54(5):469-73.

31. Chiu C-J, Mcardle AH, Brown R, Scott HJ, Gurd FN. Intestinal mucosal lesion in low-flow states. 1. A morphological, hemodynamic, and metabolic reappraisal. Arch Surg 1970;101:478-83.

32. Beckman JS, Beckman TW, Chen J, Marshall PA, Freeman BA. Apparent hydroxyl radical production by peroxynitrite: implications for endothelial injury from nitric oxide and superoxide. Proc Natl Acad Scil USA1990;87:1620-4.

33. Abello PA, Fidler SA, Bulkley GB, Buchman TG. Antioxidants modulate induction of programmed endothelial cell death (apoptosis) by endotoxin. Arch Surg 1994;129(2):134-40.

34. Bohlinger $I$, Leist $M$, Gantner $F$, Angermuller $S$, Tiegs $G$, Wendel A. DNA fragmentation in mouse organs during endotoxic shock. Am J Pathol 1996;149(4):1381-93.

35. Clavien PA, Camargo CA jr, Corczynski RM,et all. Acute reactant cytokines and neutrophil adhesion after warm ischemia in cirrhotic and noncirrhotic human livers. Hepatology $1996 ; 23(6): 1456-63$.

36. Botha Al, Moore FA, Moore EE, Sauaia A, Banerjee A, Peterson VM. Early neutrophil sequestration after injury: a pathogenic mechanism for multiple organ failure. I Trauma $1995,39(3): 411-7$.

37. Lee WL, Downey GP. Coagulation inhibitors in sepsis and disseminated intravascular coagulation. Intensive Care Med 2000;26(11):1701-6.

38. Carey MJ, Rodgers GM. Disseminated intravascular coagulation: Clinical and laboratory aspects. Am I Hematol 1998:59(1):65-73.

39. Opal S, Thijs L, Cavaillon IM, Cohen I, Fourrier F. Rouridtable I: rellationships between coagulation and inflammatory processes. Crit Care Med 2000;28(9 Suppl):S81-S82

40. Mammen EF. Antithrombin III and sepsis. Intensive Care Med 1998;24(7):649-50.

41. Balk $R$, Emerson $T$, Fourrier $F$, et al. Therapeutic use of antithrombin concentrate in sepsis. Semin Thromb Hemost 1998;24(2):183-94.

42. ten-Cate H. Pathophysiology of disseminated intravascular coagulation in sepsis. Crit Care Med 2000;28(9 Suppl):\$9-11.

43. Nijsten MW, ten Duis HI, Zijlstra JG, et al. Bllunted rise in platelet count in critically ill patients is associated with worse outcome. Crit Care Med 2000;28(12):3843-6. 
44. Fourtier $F$, Jourdain $M$, Tournois $A$, Caron $C$, Goudemand 1, Chopin $C$. Coagulation inhibitor substitution during sepsis. Intensive Care Med 1995;21:\$264-S268

45. Roback MC, Stack AM, Thompson C, Brugnara C, Schwarz HP, Saladino RA. Activated protein $C$ concentrate for the treatment of meningococcal endotoxin shock in rabbits. Shock $1998 ; 9(2)$ : $138-42$.

46. Bernard I GR, Vincent $J L$, Laterre PF, et al. Efficacy and safety of recombinant human activated protein C for severe sepsis. New Eng I Med 2001;344(10);759-62.

47. Pearson $\mid M$, Schultze AE, Schwartz KA, Scott MA, Davis IM, Roth RA. The thrombin inhibitor, hirudin, attenuates lipopolysaccharide-induced liver injury in the rat. I Pharmacol Exp Ther 1996:278(1):378-83.

48. Kondo $H_{i}$ Tani $T$, Kodama M. Effects of deletion-type human hepatocyte growth factor on murine septic model. I Surg Res 1999;85(1):88-95.

49. Ruttinger D, Vollmar B, Kempter B, Messmer $K$. Failure of Kupffer cell blockade to prevent disseminated intravascular coagulation in endotoxemic rats despite improved survival., Langenbecks Arch Surg 1998;383(1):75-80.

50. Takalla l. Splanchnic perfusion in shock [editorial]. Intensive Care Med 1994;20(6):403-4.

51. Caldini P, Permutt $S$, Waddell $\perp A$, Riley RL. Effect of epinephrine on pressure, flow, and volume relationships in the systemic circulation of dogs. Circ Res 1974;34:606-23.

52. Biffl WL, Moore EE, Moore FA. Gut-derived mediators of multiple organ failure: plateletactivating factor and interleukin-6. Br J Hosp Med 1995;54(4):134-8.

53. Kirton $\mathrm{O}$, Windsor $\mathrm{l}$, Wedderburn $\mathrm{R}$, et al. Failure of splanchnic resuscitation in the acutely injured trauma patient correlates with multiple organ system failure and length of stay in the ICU. Chest 1998;113(4):1064-169.

54. Dalton $M$ M, Gore DC, Makhoul $\mathrm{RG}_{*}$ Fisher $M R$, DeMaria El. Decreased splanchnic perfusion measured by duplex ultrasound in humans undergoing small volume hemorrhage. Crit Care Med 1995;23(3):491-7.

55. Edouard AR, Dergremont A-C, Duranteau ), Pussard E; Berdeaux A, Samil K. Heterogeneous regional vascular responses to simulated transient hypovolemia in man. Intensive Care Med $1994 ; 20: 414-20$.

56. Lam $C_{p}$ Tyml $K$, MartinC, Sibbald WJ. Microvascular perfusion is impaired in a rat model of normotensive sepsis. J Clin Invest 1994;94:2077-83.

57. Shepherd AP, Kiel JW. A model of countercurrent shunting of oxygen in the intestinal villus. Am I Physiol 1992:H1136(H1142)

58. Biffl WL Moore EE. Splanchnic ischaemia/reperfusion and multiple organ failure. $\mathrm{Br} \mathrm{J}$ Anaesth $1996 ; 77: 59-70$.

59. Cymober L. Can arginine and ornithine support gut functions? Gut 1994;Suppl 1:S42-S45

60. Bersten $A D$, Hersch $M$, Cheung $H$, Rutledge FS, Sibbald WJ. The effect of various sympathomimetics on the regional circulations in hyperdynamic sepsis. Surgery 1992;112(3): 549-61.

61. Poeze $M$, Greve JWM, Ramsay G. Is splanchnic perfusion a critical problem in sepsis? In:Baue

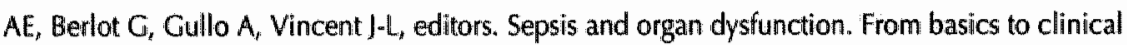
approach. 1st ed. Milano: Springer-Verlag; 1999;17, pp. 169-81.

62. Habler $O$, Kleen $M$, Hutter $\lambda_{\text {, }}$ et al. Effects of hemodilution on splanchnic perfusion and hepatorenal function. II. Renal perfusion and hepatorenal function. Eur \/ Med Res 1997;2(10): 419-24. 
63. Bitterman $\mathrm{H}$, Triolo J, Lefer AM. Use of hypertonic saline in the treatment of hemorrhagic shock. Circ Shock 1987;21(4):271-83

64. Oud $\mathbb{L}$. Kruse IA. Progressive gastric intramucosal acidosis follows resuscitation from hemorrhagic shock. Shock 1996;6(1):61-5.

65. Elizalde $\jmath_{r}$ Hernandez $\mathrm{C}_{r} \mathrm{~L}$ Llach $\mathrm{j}_{\mathrm{i}}$, et all. Gastric intramucosal acidosis in mechamically ventilated patients: role of mucosal blood flow. Crit Care Med 1998;26(5):827-32.

66. Turnage RH, Kadesky KM, Rogers T, Hernandez R, Bartula L, Myers SI. Neutrophil regulation of splanchnic blood flow after hemorrhagic shock. Ann Surg 1995;222(1):66-72.

67. Johnson DJ, Johannigman JA, Branson RD, Davis $K$, Hurst JM. The effect of low dose dopamine on gut hemodynamics during PEEP ventilation for acute lung injury. I Surg Res 1991;50:344-9.

68. Ward HB, Einzig S, Bianco RW, Wang T, Foker JE. Effects of dopamine and dobutamine on the myocardial and systemic circulation during and following cardiopulmonary bypass in dogs. Pediatr Cardiol 1982;3(3):257-64.

69. Chintala MS, Moore RJ, Lokhandwala MF, Jandyala BS. Evaluation of the effects of dopexamine, a novel DA1 receptor and b2-adrenoreceptor agonist, on cardliac function and splanchnic circulation in a canine model of hemorrhagic shock. Arch Pharmacol 1993;347:296-300.

70. Donati $A$, Battisti $D$, Rechionii $A$, et al. Predictive value of interleukin-6 (UL-6), interleukin$8(\mathrm{LL}-8)$ and gastric intramucosal $\mathrm{pH}(\mathrm{pH}-\mathrm{i})$ in major abdominal surgery. Intensive Care Med 1998;24(329):335

71. Welte $M$, Pichler $B$, Groh J,et al. Perioperative mucosal $\mathrm{pH}$ and splanchnic endotoxin concentration in orthotopic liver transplantation. Br | Anaesth 1996;76(1):90-8.

72. Bacher A, Mayer N, Rajek AM, Haider W. Acute normovolemic haemodilution does not aggrevate gastric mucosal acidosis during cardiac surgery. Intensive Care Med 1998;24:313-21.

73. Chang $M C_{r}$ Meredith JW. Cardiac preload, splanchnic perfusion, and their relationship during resuscitation in trauma patients. J Trauma 1997;42(4):577-82.

74. Maynard ND, Bihari D, Beale R, et al. Assessment of splanchnic oxygenation by gastric tonometry in patients with acute circulatory failure. JAMA 1993;270(10):1203-10.

75. Mythen $M G_{1}$, Webb AR. Perioperative plasma volume expansion reduces the incidence of gut mucosal hypoperfusion during cardiac surgery. Arch Surg 1995;130(4):423*9.

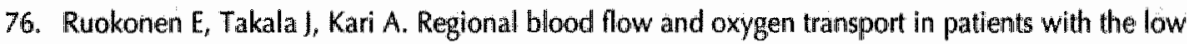
cardiac output syndrome after cardiac surgery. Crit Care Med 1993;21(9):1304:11.

77. Kainuma $M$, Kimura $N$, Nonami $T$, et al. The effect of dobutamine on hepatic blood flow and oxygen supply-uptake ration during enflurane nitric oxilde anesthesia in humans undergoing liver resection. Anaesthesiology 1992;77:432-8.

78. Kaisers U, Pappert $D_{s}$ Langrehr JM, Undi H, Neuhaus P, Rossaint R. Dopamine, dopexamine and dobutamine in liver transplant recipients: a comparison of their effects on hemodlynamics, oxygen transport and hepatic venous oxygen saturation. Transpl Int 1996;9:214-20.

79. Takala J. Determinants of splanchnic blood flow. Br I Anaesth 1996;77:50-8.

80. Ince $C$, van der Sluïgs. JP, Sinaasappel $M$, Avontuur $\ A$, Coremans JM, Bruining HA. Intestinal ischemia during hypoxia and experimental sepsis as observed by $\mathrm{NADH}$ videofluorimetry and quenching of Pd-porphine phosphorescence. Adv Exp Med Biol 1994;361:105-10.

81. Pastores $S M, K a t z$ DP, Kwetan V. Splanchnic ischemia and gut mucosal injury in sepsis and the multiple organ dysfunction syndrome. Am \ Gastroenterol 1996;91 (9):1697-710.

82. Turnbull RG, Talbot JA, Hamilton SM. Hemodynamic changes and gut barrier function in sequential hemorrhagic and endotoxic shock. I Trauma 1995;38(5):705-13. 
83. Fink MP, Rothschild HR, Deniz YF, Wang HL, Lee PC, Cohn SM. Systemic and mesenteric $\mathrm{O}_{2}$ metabolism in endotoxic pigs: Effects of ibuprofen and meclofenamate. I Appl Physiol 1989;67:1950-7.

84. De Backer $\mathrm{D}$, Zhang $\mathrm{H}_{3}$ Manikis $\mathrm{P}$, Vincent $\mathrm{J}-\mathrm{L}$. Regional effects of dobutamine in endotoxic shock. I Surg Res 1996;65(2):93-100.

85. Dahn MS, Lange MP, Wilson RF, lacobs $L A$, Mitchell RA. Hepatic blood flow and splanchnic oxygen consumption measurements in clinical sepsis. Surgery 1990;107(3):295-301.

86. Dahn MS, Lange P, Lobdell K; Hans B, Jacobs LA, Mitchell RA. Splanctnic and total body oxygen consumption differences in septic and injured patients. Surgery 1987;101(1):69-80.

87. Ruokonen E, Takala J, Kari A, Saxen H, Mertsola J, Hansen E). Regionall blood flow and oxygen transport in septic shock. Crit Care Med 1993;21:1296-303.

88. Friedman $\mathrm{C}$, Berlot $\mathrm{G}$, Kahn $\mathrm{R}$, Vincent J-L. Combined measurements of blood lactate concentrations and gastric intramucosal pH in patients with severe sepsils. Crit Care Med 1995;23(7):1184-93.

89. Olson D, Pohlman A, Hall JB. Administration of low-dose dopamine to nonoliguric patients with sepsis syndrome does not raise intramucosal gastric $\mathrm{pH}$ nor improve creatinine clearance. Am J Respir Crit Care Med 1996;154:1664-70.

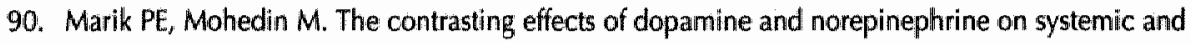
splanchnic oxygen utilization in hyperdynamic sepsis. JAMA 1994;272(17):1354-7.

91. Ruokonen E, Takala J, Kari A, Saxen H, Mertsolla J, Hansen E. Regional blood flow and oxygen transport in septic shock. Crit Care Med 1993;21(9):1296-303.

92. Levy $\mathrm{B}$, Bollaert $\mathrm{PE}$, Charpentier $\mathrm{C}_{s}$ ef al. Comparison of norepinephrine and dobutamine to epinephrine for hemodynamics, lactate metabolism, and gastric tonometric variables in septic shock: a prospective, randomized study. Intensive Care Med 1997;23(3):282-7.

93. Gutierrez $\mathrm{G}$, Palizas $\mathrm{F}$, Doglio $\mathrm{G}_{\text {r }}$ et al. Castric intramucosal $\mathrm{pH}$ as a therapeutic index of tissue oxygenation in critically ill. Lancet 1992;339(1):195-9.

94. Giraud GD, MacCannell KL. Decreased nutrient blood flow during dopamine- and epinephrineinduced intestinal vasodilatation. \Pharmacol Exp Ther 1984;230:214-20.

95. Rombeau JL, Takala J. Summary of round table conference: gut dysfunction in critical illness. Intensive Care Med 1997;23:476-9.

96. Reynolds IV, Murchan P, Redmond HP, et al . Failure of macrophage activation in experimental obstructive jaundice: association with bacterial translocation. Br J Surg 1995;82(4):534-8.

97. Agalar F, Iskit AB, Agalar C, Hamaloglu $E$, Guc MO. The effects of G-CSF treatment and starvation on bacterial translocation in hemorrhagic shock. I Surg Res 1998;78(2):143-7.

98. Deitch EA, Morrison J, Berg R, Specian RD. Effect of hemorrhagic shock on bacterial translocation, intestinal morphology, and intestinal permeability in conventional and antibioticdecontaminated rats. Crit Care Med 1990; 18(5):529-36.

99. Deitch EA, Berg R, Specian R. Endotoxin promotes the translocation of bacteria from the gut. Arch Surg 1987;122:185-90.

100. Alexander JW, Boyce ST, Babcock $\mathrm{CF}_{t}$ et al. The process of microbial translocation. Ann Surg $1990 ; 212(4): 496-511$.

101. Lemaire LCIM, van Wagensveld BA, van Gulik ThM, Dankert J, van Lanschot JJB, Gouma D]. Bacterial translocation to the thoracic duct in a setting of ischemia partial resection and reperfusion of the porcine liver. Dig Surg 1999; 16(3):222-8.

102. Moore FA, Moore EE, Poggetti R, MCAnena O], Peterson VM, Abernathy CM, Parsons PE. Gut bacterial translocation via the portal vein: a clinical perspective with major torso trauma. I Trauma $1991 ; 31(5): 629-36$. 
103. Olofsson P, Nylander $G$, Ollson P. Endotoxin: routes of transport in experimental peritonitis... Am J Surg 1986;151(4):443-6.

104. Lemaire $L_{\text {, }}$ van Deventer SJ, van Lanschot JJ, Meenan J, Gouma DJ. Phenotypical characterization of cells in the thoracic duct of patients with and without systemic inflammatory response syndrome and multiple organ failure. Scand I Immunol 1998;47(1): 69-75

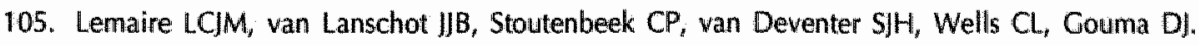
Bacterial translocation in multiple organ failure: cause or epiphenomenon still unproven. $\mathrm{Br}$ ) Surg 1997;84:1340-50.

106. Poeze $M$, Greve JWM, Ramsay $G$. Gut decontamination - prevention of translocation. In: Baue AE, Fry DE, editors. SIRS, MODS and MOF - Systemic inflammatory response syndrome, multiple organ dysfunction syndrome and multiple organ failure - pathophysiology, prevention and therapy. 1st ed. Berlin: Springer-Verlag; 2000; 59, pp. 580-90.

107. Jarvis WR. The epidemiology of colonization. Infect Control Hosp Epidemiol 1996;17(1):47-52.

108. Millership SE, Patel $\mathbb{N}_{v}$ Chattopadhyay $B$. The colonization of patients in an intensive treatment unit with Gram-negative fllora: the significance of the oral route. $\|$ Hosp Infect 1986;7:226-35.

109. Nyström $B_{x}$ Frederici $H$, von Euler $C$. Bacterial colonization and infection in an intensive care unit. Intensive Care Med 1988;14:34-8.

110. Mason $\mathrm{CM}_{x}$ Dobard E, Summer WR, Nelson S. Intraportal lipopolysaccharide suppresses pulmonary antibacterial defense mechanisms. J Infect Dis 1997;176(5):1293-302.

111. Leeuwen van $P A M_{*}$ Boermeester $M A_{s}$ Houdijk APJ., et al. Clinical significance of translocation. Gut 1994; Suppl 1:S28-S34

112. Hartung T, Sauer A, Hermann $C$, Brockhaus F, Wendel A. Overactivation of the immune system by translocated bacteria and bacterial products. Scand J Gastroenterol Suppl 1997;222:98-9.

113. Hartung $T$, Sauer $A_{*}$ Hermann $C_{1}$ Brockhaus $F$, Wendel $A$. Overactivation of the immune system by translocated bacteria and bacterial products. Scand J Gastroenteral Suppl 1997;222:98-9.

114. Ayala $A$, Song GY, Chung $C S$, Redmond $\mathrm{KM}_{*}$ Chaudry IH. Immune depression in polymicrobial sepsis: the role of necrotic (injured) tissue and endotoxin. Crit Care Med 2000;28(8):2949-55.

115. Arai $\mathrm{KI}$, Lee $\mathrm{F}$, Miyajima $\mathrm{A}$, Miyatake S, Arai $\mathrm{N}$, Yokota T. Cytokines: coordinators of immune and inflammatory responses. Annu Rev Biochem 1990;59:783-836.

116. Streiter $R M$, Kunkel $S$, Bone $R C$. Role of tumor necrosis factor-a in disease states and inflammation. Crit Care Med 1993;21 (10):\$447*5463

117. Camussi $\mathrm{G}$, Albano $\mathrm{E}$, Tetta $\mathrm{C}$, Bussolino $\mathrm{F}$. The molecular action of tumor necrosis factor " ${ }^{\text {. Eur }}$ I Biochem 1991;202:3-14.

118. Schirmer WJ, Schirmer JM, Fry DE. Recombinant human tumor necrosils factor produces hemodynamic changes characteristic of sepsis and endotoxemia. Arch Surg 1989;124(4):445-8.

119. van der Poll $T$, Buller $H R$, ten-Cate $H_{\text {, }}$ et al. Activation of coagulation after administration of tumor necrosis factor to normal subjects. New Eng / Med 1990;322(23):1622-7.

120. Aderka $D$, Sorkine $P$, Adu-Adib $S$, et al. Shedding kinetics of soluble tumor necrosis factor (TNF) receptor after systemic TNF leaking during isolated limb perfusion. Relevance to the pathophysiology of septic shock. . Clin Invest 1998;101(3);650-9.

121. Heinrich $P C$, Castell JV, Andus T. Interleukin-6 and the acute phase response. Biochem I 1990;265(3):621-36.

122. Castell NV, Gomez-Lechon MJ, David M,et al. Interleukin 6 is the major regulator of acute phase protein synthesis in aduilt human hepatocytes. FEBS Lett 1989;242(2):237-9.

123. Yao YM, Bahrami $S$, Redl $H$, Fuerst $S$, Schlag G. IL-6 release after intestinal ischemia/reperfusion in rats is under partial control of TNF. I Surg Res 1997;70(1):21-6. 
124. Libert $C$, Vink $A$, Coulie $P$, et al. Limited involvement of interleukin-6 in the pathogenesis of lethal septic shock as revealed by the effect of monocloncal antobodies against interleukin-6 or its receptors in various murine models. Eur I Immunol 1992;22:2625-30.

125. Baggiolini $M$, Dewald B, Moser B. Human chemokines: an update. Annu Rev Immunol 1997;15: 675-705.

126. Carpenter $A$, Evans $T$, Biumunan WA, Bemelmans MHA, Moyes $D$, Cohen J. Differences in the shedding of soluble TNF-receptors between endotoxin-sensitive and endotoxin-resistant mice in response to lipopolysaccharide or live bactierial challenge. J Immunol 1995;155(4): 2005-12.

127. Engelberts 1 , Stephens $S$, Francot GJM, van der Linden Cl, Buurman WA. Evidence for different effects of soluble TNF-receptors on various TNF measurements in human biological fluids. Lancet $1991 ; 338: 515-6$.

128. Aderka D, Engelmann $H$, Maor $Y$, Brakebusch $C$, Wallach D. Stabilization of the biaactivity of tumor necrosis factor by its soluble receptors. I Exp Med 1992;175:323-9.

129. van Zee KJ, Kohno T, Fischer E, Rock CS, Moldawer LL, Lowry SF. Tumor necrosis factor soluble receptors circulate during experimental and clinical inflammation and can protect against excessive tumor necrosis factor alpha in vitro and in wivo. Proc Natl Acad Sci USA 1992;89(11): 4845-9.

130. van der Poll T, Coyle SM, Levi $M$, et al. Effect of a recombinant dimeric tumor necrosis factor receptor on inflammatory responses to intravenous endotoxin in normal humans. Blood 1997;89:10;:3727-34.

131. Bouma MG, Laan MP, Dentener MA, et al. Analysis of soluble adhesion molecules. In: Johnstone AP, Turner MW, editors. limmunochemistry 2: A practicall approach. 1st ed. Oxford: Oxford University Press; 1997; 7, pp. 181-96.

132. Stad RK, Buturman WA. Current views on structure and function of endothelial adhesion molecules. Cell Adhesion Comm 1994;2:261-8.

133. Dustin ML, Rothlein $R$, Bhan AK, Dinarello CA, Springer TA. Induction by IL 1 and interferongamma: tissue distribution, biochemistry, and function of a natural adherence molecule (ICAM1). J Immunol 1986;137(1):245-54.

134. Ogle CK, Mao JX, Hasselgren $\mathrm{PO}_{v}$ Ogle JD, Alexander IW. Production of cytokines and prostaglandin $\mathrm{E} 2$ by subpopulations of guinea pig enterocytes: effect of endotoxin and thermal injury. I Trauma 1996;411(2):298-305.

135. Ogle CK, Guo X, Hasselgren $\mathrm{PO}$, Ogle JD, Alexander JW. The gut as a source of inflammatory cytokines after stimulation with endotoxin. Fur I Surg 1997; 63(11);45-51.

136. Ayala $A_{i}$ Perrin $M M_{t}$ Meldrum $D R$, Ertel $W$, Chaudry $\mathbb{H}$. Hemorthage induces an increase in serum TNF which is not associated with elevated levels of endotoxin. Cytokine 1990;2:170-4.

137. Chaudry $1 \mathrm{H}$, Ayala $\mathrm{A}$. Mechanism of increased susceptibility to infection following hemorrhage. Am I Surg 1993;165(2A (Suppl)):595-67S.

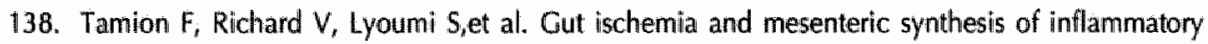
cytokines after hemorrhagic or endotoxic shock. Am I Physiol 1997;273(36):G314-G321

139. Deitch $E A, X u D$, Franko $L$, Ayala $A$, Chaudry $\| H$. Evidence favoring the role of the gut as a cytokine-generating organ in rats subjected to hemorrhagic shock. Shock 1994;1(2):141-5.

140. Guo W, Ding J, Huang Q, Jerrelis T, Deitch EA. Alterations in intestinal bacterial flora modulate the systemic cytokine response to hemorrhagic shock. Am J Physiol 1995;269(32): G827-G832

141. Cabie A, Farkas JC, Fitting $C$, et al. High levels of portal TNF-alpha during abdominal aortic surgery in man. Cytokine 1993:5(5):448-53. 


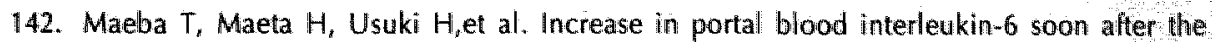
commencement of digestive surgery. Surg Today 1996;26(11):890-4.

143. Towfigh $S$, Heisler $T$, Rigberg $D A$, et al. Intestinal ischemia and the gut-liver axis: an in vitro model. I Surg Res 2000;88(2):160-4.

144. McGuinness OP, Lacy DB, Ejiofor J, Bagby GG. Hepatic release of tumor necrosis factor in the endotoxin-treated conscious dog. Shock 1996;5(5);344-8.

145. Koo DJ, Chaudry IH, Wang P. Kupffer cells are responsible for producing inflammatory cytokines and hepatocellular dysfunction during early sepsis. I Surg Res 1999;83(2):151-7.

146. Ayala $A$, Perrin $M M$, Wang $P$, Chaudry $I H$. Sepsis induces an early increased spontaneous release of hepatocellular stimulatory factor (interleukin-6) by Kupffer cells in both endotoxin tolerant and intolerant mice. I Surg Res 1992;52,6):635-41.

147. Ayala A, O'Neill Pl, Uebelle SA, Herdon CD, Chaudry IH. Mechanism of splenic immunosuppression during sepsis: key role of Kupffer cell mediators. I Trauma 1997;42(5): $882-8$.

148. Douzinas EE, Tsidemiadou PD, Pitaridis MT, et al. The regional production of cytokines and llactate in sepsis-related multiple organ failure. Am I Respir Crit Care Med 1997;155:53-9.

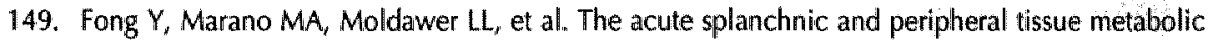
response to endotoxin in humans. J Clin Invest 1990;85:1896-904.

150. Avontuur JA, Bruining HA, Ince C. Sepsis and nitric oxide. Adv Exp Med Biol 1996;388:551 67.

151. Gomez-Jimenez J, Salgado $A_{*}$, Mourelle $M$, Martin MC, Segura RM, Peracaula $R$, Moncada S. L-arginine: nitric oxide pathway in endotoxemia and human septic shock. Crit Care Med 1995;23(2):253-8.

152. Ferraz JG, Tigley $A$, Wallace IL. Paradoxical effects of L-arginine on gastric mucosal integrity. Eur J Pharmacol 1994;260(1):107-11.

153. Ruetten $H$, Southan G], Abate A, Thiemermann $C$. Attenuation of endotoxin-induced multiple organ dysfunction by 1-amino-2-hydroxy-guanidine, a potent imhibitor of inducible nitric oxide synthase. Br I Pharmacol 1996;118(2):261-70.

154. Minnard EA, Shou J, Naama $H$, Cech A, Gallagher $H$, Daly IM. Inhibition of nitric oxide synthesis is detrimental during endotoxemia. Arch Surg 1994;129(2):142-7.

155. Grover R, Lopez A, Lorente $\| A_{\text {, }}$ et al. Multi-center, randomized, placebo-controlled, double blind study of the nitric axide synthase inhibitor $546 \mathrm{C} 88$ : effect on survival in patients with septic shock. [Abstract] Crit Care Med 1998;27:(1)A33

156. Spain DA, Wilson MA, Bar-Natan MF, Garrison RN. Nitric oxide synthase inhibition aggrevaltes intestinal microvascular vasoconstriction and hypoperfusion of bacteremia. J Trauma 1994;36(5): 720-5.

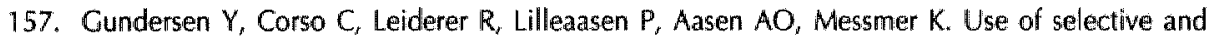
nonselective nitric oxide synthase inhibitors in rat endotoxemia: effects on hepatic morphaology and function. Shock 1997;8(5):368-72.

158. Corso C, Gundersen Y, Messmer $K$, Lillealasen P, Aasen AO, Messmer. Effects of the nitric oxide synthase inhibitors NG-nitro-L-arginine methyl ester and aminoethyl-isothiourea on the liver microcirculation in rat endotoxemia. I Hepatol 1998;28:61-9.

159. Florquin $S$, Amraoui $Z_{x}$ Dubois $C_{s}$ Decuyper $J_{x}$ Goldman $G$. The protective role of endogenously synthesized nitric oxide in staphylococcal enterotoxin B-induced shock in mice. I Exp Med 1994; 180:11:153-8.

160. Wray $\mathrm{GM}$, Millar CC, Hinds $\mathrm{Cl}$, Thiemermann C. Selective inhibition of the activity of inducible nitric oxide synthase prevents the circulatory failure, but not the organ injury/dysfunction, caused by endotoxin. Shock 1998;9(5):32:9-35. 
161. Wang $Y$, Lawson IA, Jaeschike H. Differential effect of 2-aninoethy-isotiourea, an inhibitor of the inducible nitric oxide synthase, on microvascular blood flow and organ injury in models of hepatic ischemia-reperfusion and endotoxemia. Shock 1998; 10(1):20-5.

162. Saetre $T_{r}$ Gundersen $Y$, Thiemermann $C$, Lilleaasen $P$, Aasen AO. Amino-isothiourea, a selective inhilbitor of inducible nitric oxide synthase activity, improwes liver circulation and oxygen metabolism in a porcine madel of endotoxemia. Shock 1998;9(2):109-15.

163. Strand $\varnothing A$, Kirkebøen KA, Giercksky K-E. Inthibition of nitric oxide synthase does not affect survival in a rat model of abdominal sepsis. Scand I Clin Lab Invest 1997,57:105-10.

164. Bruins M), Soeters PB, Lamers WH, Meijer A), Deutz NE. L-arginine supplementation in hyperdynamic endotoxemic pigs: effect on nitric axide synthesis by the different ongans. Crit Care Med 2002;30(3):508-517.

165. Angele MK, Smail $N_{x}$ Wang $P_{x}$ Cioffi WG, Bland $K I$, Chaudry $I H$. L-arginine restores the depressed cardiac output and regional perfusion after trauma-hemorrhage. Surgery 1998;124(2);394-401.

166. Gianotti L, Alexander JW, Pyles T, Fukushima R. Arginine-supplemented diets improve survival in gut-derived sepsis and peritonitis by modulating bacterial clearance. The role of nitric oxide. Ann Surg 1993;217(6):644-54. 


\section{SUMMARY}

Since the introduction of invasive monitoring technology, numerous new applications have been developed. These new techniques, focussing on global, organ or cellular level, are related to a change in focus with regard to the parameters that should be used, resulting from increasing knowledge of the pathophysiology of shock and organ failure. Both experimental and clinicall studies have been conducted to test the validity and reproducibility of these techniques. 42. A limited number of techniques have been tested in randomised clinical trials. This review discusses the newer monitoring techniques which can be used on the intensive care unit and gives an overview concerning new emerging monitoring techniques. 


\section{INTRODUCTION}

Haemodynamic monitoring is one of the major reasons for patients to be admitted to an intensive care unit. The first patients to be monitored and treated at an intensive care unit were those involved in the polio epidemic in the 1920's (1). Since then, major advances have been made in monitoring critically ill patients, especially after the introduction of intravascular pressure- and flow-recording catheters in the 1970s (1). Nowadays, intensivists frequently use invasive haemodynamic monitoring instruments, including intra-arterial, pulmonary artery and central venous catheters, to guide therapeutic interventions. Recent studies have recommended that intensivists should use less invasive modalities, because of the complications related to the use of invasive techniques, such as thrombosis and catheter-related sepsis (2-5).

Studies aiming at improving the outcome of critically ill and surgical patients have emphasised the importance of better monitoring, better use of monitoring and early use of monitoring techniques (6). It seems justified to use more invasive haemodynamic monitoring in patients who do not yet have complications, but are at increased risk of developing them. However, if additional monitoring techniques are to be more generally applied, a balance should be found between more invasive monitoring techniques, which provide additional information, but also increase the risk of complications, and less invasive techniques, which produce fewer complications but in theory also less accurate haemodynamic information.

Progress in monitoring technology made over the last decades has been related to a change in focus with regard to the parameters that should be used, resulting from increasing knowledge of the pathophysiology of shock and organ failure. Whereas monitoring used to be aimed at measuring blood pressure and heart rate, the introduction of invasive monitoring techniques changed this focus to monitoring cardiac output and pressure-related parameters (wedge pressure) and derived parameters (systemic vascular resistance), these refer to the global level of monitoring. Pathophysiological studies subsequently focused on shock as a dysbalance between oxygen consumption and oxygen delivery in the tissues, rather than just low blood pressure (7). Concentrating on oxygen delivery and consumption as the goal of haemodynamic optimisation, instead of pressure-related parameters, changed the focus to measuring oxygen delivery and oxygen consumption (8). As was explained in the previous chapter, some organ systems are more vulnerable to such a dysbalance than others. Attention thus shifted to the dysfunction of individual organs during sepsis and shock (i.e., the organ level), with the function of the hepatosplanchnic organs in particular being used as a monitoring tool. Numerous techniques to assess the functioning and perfusion of the hepatosplanchnic organs were developed.

More recent developments have been concentrating on the assessment of the microcirculation and mitochondrial function during sepsis and organ failure (cellular level) (9). Techniques for monitoring these systems have been developed, but have not been as intensively validated for clinical use in surgical and septic patients and will not be discussed in this chapter. 
This chapter discusses some of these monitoring techniques at several levels, from the global to the organ level. This discussion concentrates on the use of both minimally invasive and regional monitoring techniques, and addresses both technical aspects and the clinical studies published so far in light of the studies reported on in the present thesis.

\section{MONITORING MODALITIES}

\section{Global monitoring level}

\section{Invasive: Pulmonary Artery Thermodilution Catheter (PAC)}

Pulmonary artery or Swan-Ganz catheterisation was first used in 1970 in patients with acute heart disease. Since then, this technique has gained widespread use in both cardiac and non-cardiac diseases. Pulmonary artery catheterisation (PAC) provides global haemodynamic information with accurate assessment of the cardiac output and left ventricular filling pressures. The use of these variables has led to new insights into the pathophysiology of shock.

The PAC, which has a balloon at the tip of the catheter, can be advanced through the subclavian, jugular, brachial or femoral veins. The tip of the PAC lies in the pulmonary artery and closes off one of the branches of this artery when the balloon is inflated (wedge position).

The main variables which can be measured using the PAC are the stroke volume, using a cold saline bolus and a temperature sensor at the tip of the catheter, pulmonary artery pressure (PAP), central venous pressure (CVP) and pulmonary artery wedge pressure or pulmonary artery occlusion pressure (PCWP), using the balloon at the tip of the catheter. The other variables that can be assessed with PAC are derived variables and include cardiac output (CO), systemic and pulmonary vascular resistance (SVR and PVR), left ventricle stroke work index (LVSWI) and right ventricle stroke work index (RVSWI).

Many studies have used parameters measured with the Swan-Ganz catheter as the "gold standard", the method with which all other methods are compared. Despite this, the invasive nature of the PAC technique, as well as the uncertain validity of the derived parameters, has led to dissatisfaction with this method. The complications associated with PAC include thrombosis $(0 \%-1.4 \%)$, arrhythmia (1.3-1.5\%), pneumothorax (1\%), and catheter-related sepsis (1\%) (2-4). Moreover, a recent retrospective study by Connors et al. suggested that the use of pulmonary artery catheters was associated with a significant increase in mortality, via mechanisms as yet undefined (5). Subsequently, other techniques were developed, using less invasive or completely non-invasive methods to determine the cardiovascular status. 
Other methods have also been deployed to improve the application of the thermodilution method, viz. continuous heat-induced thermodilution and 'injection-less' cold thermodilution (1 0,11 . Continuous heat-induced thermodilution uses the traditional thermodilution technique, but uses conduction of heat instead of cold and of energy instead of fluid. The part of the catheter in the right ventricle is wrapped with heating filaments. These filaments release heat into the circulation in a computer-controlled repetitive on-off sequence. These data are computerised by cross-correlating the input sequence and the measured temperature changes, thereby creating a classical thermodilution curve. The method measures cardiac output continuously, is less influenced by arrhythmia and ventilation, and reduces the volume added during the measurements. The 'injection-less' cold thermodilution technique also reduces the volume of fluids added (10). The catheter has an extra balloon proximally on the catheter, into which cold fluid can be injected. After the cold wave has spread from the balloon, the fluid can be withdrawn. This technique has significantly reduced the risk of infection.

\section{Invasive: Double indicator dilution method}

\section{Technique}

The thermal-dye dilution method (double-indicator dilution method) uses two different injected indicators, i.e. a bolus of ice-cold water and a bolus of indocyanine green (ICG) to create dilution curves. The indicators are injected simultaneously into a central venous site, similar to the Swan-Ganz thermodilution method. The dilution is measured at one point in the arterial circulation. Usually for this purpose a specialised fiberoptic-thermistor catheter is usually inserted through the femoral artery into the descending aorta. The fiberoptic catheter is connected to a fiberoptic densitometer module, which measures ICG light absorption at a wavelength of $805 \mathrm{~nm}$. Studies comparing the thermodilution and dye-dilution method have found good correlations for cardiac output values (12). The principle of the double indicator dilution method is that an indicator injected into a central vein always mixes with the greatest volume available and that it is not possible for the indicator to re-concentrate after relevant dilution. The ice-cold water bolus mingles with the end-diastolic volumes of the right atrium and is then transported to the right ventricle, where it dilutes further into the right-ventricular end-diastolic volume (Figure 1). The injected indicator then continues through the lungs, the end-diastolic volume of the left atrium and the ventricle. The volume of the vessels between the heart and the lungs play a minor rolle as volume constant in the calculations. The cold bolus can be used to determine the extravascular volumes. As a result of heat conduction (by convection), the cold saline bolus can also distribute to the extravascular space, depending on the surface and time available for exchange. As the heat exchange area in the lungs is more than 1000 times larger than that of the heart chambers and large vessels, the cold wave also spreads into the extra-vascular volume of the lungs. In contrast, the ICG dilutes intravascularly only, and subtracting the volumes measured by the two indicators allows the difference between intra-vascular and extra-vascular volumes to be calculated (Figure 1). 


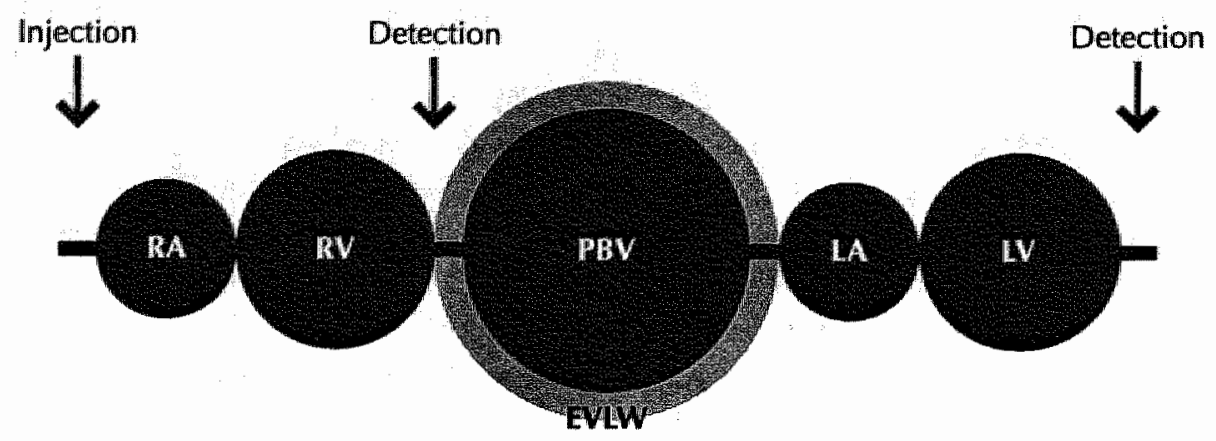

This diagram depicts the flow trajectory of the cold indocyanine green $\| \mathrm{CO}$ bolus through the cardiopulmonary system. The ICG is injected before the right atrium (RA), after which it fllows through the right ventricle (RV), pulmonary bilond volume (PBV), Heft atrium (LA), and left wentricle (LV). The ICG remains intravascular, but the cold bolus extends into the extravascular lung space. The extravascular lung water wolume (EVLW) can be calculated by subtracting the volume measured by the ICG method fron the total wolume measured. Measurement sites are the pulmonary artery and the femoral artery. This enables the various volumes and candiac output to be calculated (see figure 2).

Figure 1 Schematic depiction of the mixing chambers in the cardiopulmonary system

ICG is a non-toxic and well-tolerated dye that binds, immediately after injection into the biood stream, to plasma proteins with a molecular weight of $>70,000$ kD. More than $99.9 \%$ of these plasma proteins remain intravascular, even in the presence of severe capillary leakage.

Besides cardiac output, specific volumes can be calculated from each indicator's dilution using the measured mean transit time (MTt) and the exponential downslope time (DSt) (Figure 2). MTt is composed of the appearance time At (the time between injection and the moment of the first appearance of the indicator) and the mean time difference between the occurrence of the first indicator and the completion of the indicator flow:

$M T t=A t+\frac{\int c \cdot(t-A t) \cdot d t}{\int c i \cdot d t}$

The resultant of the cardiac output $(\mathrm{CO})$ and MTt is the volume covered by the indicator, i.e. the volume between the place of injection and the place of measurement, and the resultant of the CO and DSt represents the volume of the largest mixing chamber. Measuring the MTt from the thermodilution curve in the pulmonary artery (Tdpa) and multiplying this with the cardiac output measured at this site allows the right heart end-diastolic volumes to be calculated. 

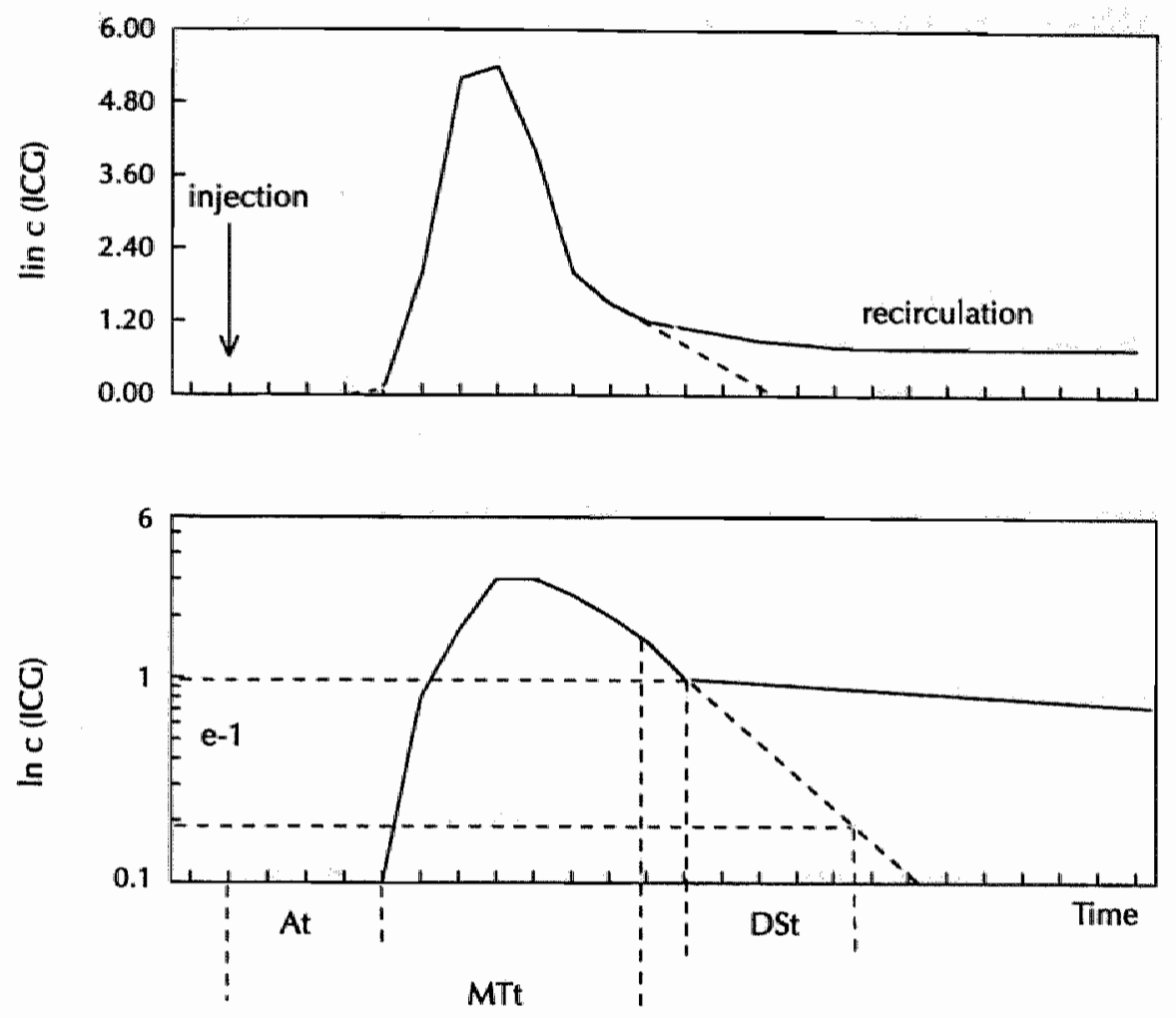

Specific volumes can be calcullated by multiplying the cardiac output by the characteristic tramsit times: the mean transit times (MTt) and the exponential downslope time (DSt). The resultant of the CO and the MTt is the volume covered by the relevant indicator, i.e. the volume between the place of injection and the place of measurement. The resultant of the CO and DSI is the volume of the largest mixing chamber in the lemperature distance covered by the indicator. For example: $\mathrm{PBV}=\mathrm{DST}$ dye-dilution arterial times arterial $\mathrm{CO}$ thermodilution .

Figure 2 Schematic depiction of the dilution curves and their analysis for transit times

\section{Previous studies}

Two experimental studies in piglets have assessed the accuracy and reproducibility of the circulating blood volume measurement $(13,14)$. The first of these studies compared total blood volume measurements using ICG with measurements using Evans blue, and found a good correlation $\left(r^{2}=0.83\right)$. The other study examined the relation between cardiac output and intrathoracic blood volume (ITBV) during normovolaemia, hypovolaemia, and hypervolaemia, and found a good correlation between ITBV and the stroke volume in all conditions. This good correlation was confirmed in critically ill patients. In this study, ITBV and the other preload 
dependent variables CVP and PCWP were compared with preload independent variables $\mathrm{Cl}_{1} \mathrm{DO}_{2}$ and $S V R$. The authors concluded that ITBV is a suitable indicator of circulating blood volume. In another study in critically ill patients, Haller et al. found an excellent correlation between cardiac output values measured using standard thermodilution and ICG dilution $\left(r^{2}=0.91\right)(15)$.

Indocyanine green allows plasma volumes to be measured independently of the disappearance rate from plasma in critically ill patients, unless there is a severe generalised capillary protein leakage (16). The latter may arise as a result of microvascular damage in the lungs during severe sepsis and inflammation. The protein leakage attracts fluid into the interstitium of the lungs, thereby increasing the amount of extravascular water in the lung. Fluid management during critical illness must be aimed at improving perfusion, but in severe inflammation, there is the potential disadvantage that major fluid shifts may occur to the extravascular space. Measuring extravascular lung water may be important to optimise the balance between the amount of infusion needed for adequate perfusion and the amount of fluid which can induce failure of the heart. Extravascular lung water (EVLW) can be adequately assessed using the double indicator dilution method. Fluid management in critically ill patients using EVLW as a parameter has been found to improve outcome (in terms of fewer ventilator days and length of ICU stay) compared to using CVP. This was associated with a decrease in EVLW in the EVLW-guided treatment group (17). The predictive value of these double indicator dilution parameters is not known at present.

\section{Non-invasive: Doppler ultrasound}

Technique

The Doppler monitor, which was developed in the early 1970 s, provides a safe and minimally invasive means of continuouslly monitoring global haemodynamic status. The Doppler probe is inserted into the oesophagus (about the size of a nasogastric tube), in the trachea or in the suprasternal notch and measures the blood flow velocity in the descending or ascending aorta.

Blood flow velocity measurements using ultrasound are based on the Doppler principle: ultrasound is reflected by moving objects (red blood cells) moving towards the transmitted signal, causing reflections of a higher frequency. This Doppler shift ( $\triangle F$ ) can be described by:

$$
\Delta \mathrm{F}=2 . f_{\mathrm{T}} \cdot \mathrm{v} . \frac{\cos \theta}{\mathrm{s}} \quad \mathrm{v}=\frac{2 \cdot \Delta \mathrm{F}}{f_{\mathrm{T}} \cdot \cos \theta}
$$

The velocity ( $v$ ) can be calculated using the known $\Delta F(\mathrm{~Hz})$, the transmitted frequency $\left(f_{T}\right)$, the speed of the ultrasound in the medium $(s ; 1,540 \mathrm{~m} / \mathrm{sec}$ in body tissue) and the angle between the ultrasound beam and the direction of the object's motion $(\cos \theta)$. Blood flow is usually measured using transmitted frequencies in the 2 to $16 \mathrm{MHZ}$ range. In the suprasternal approach, the ultrasound beam is assumed to be in line with the aortic flow, avoiding any angle effect. Correct placement is 


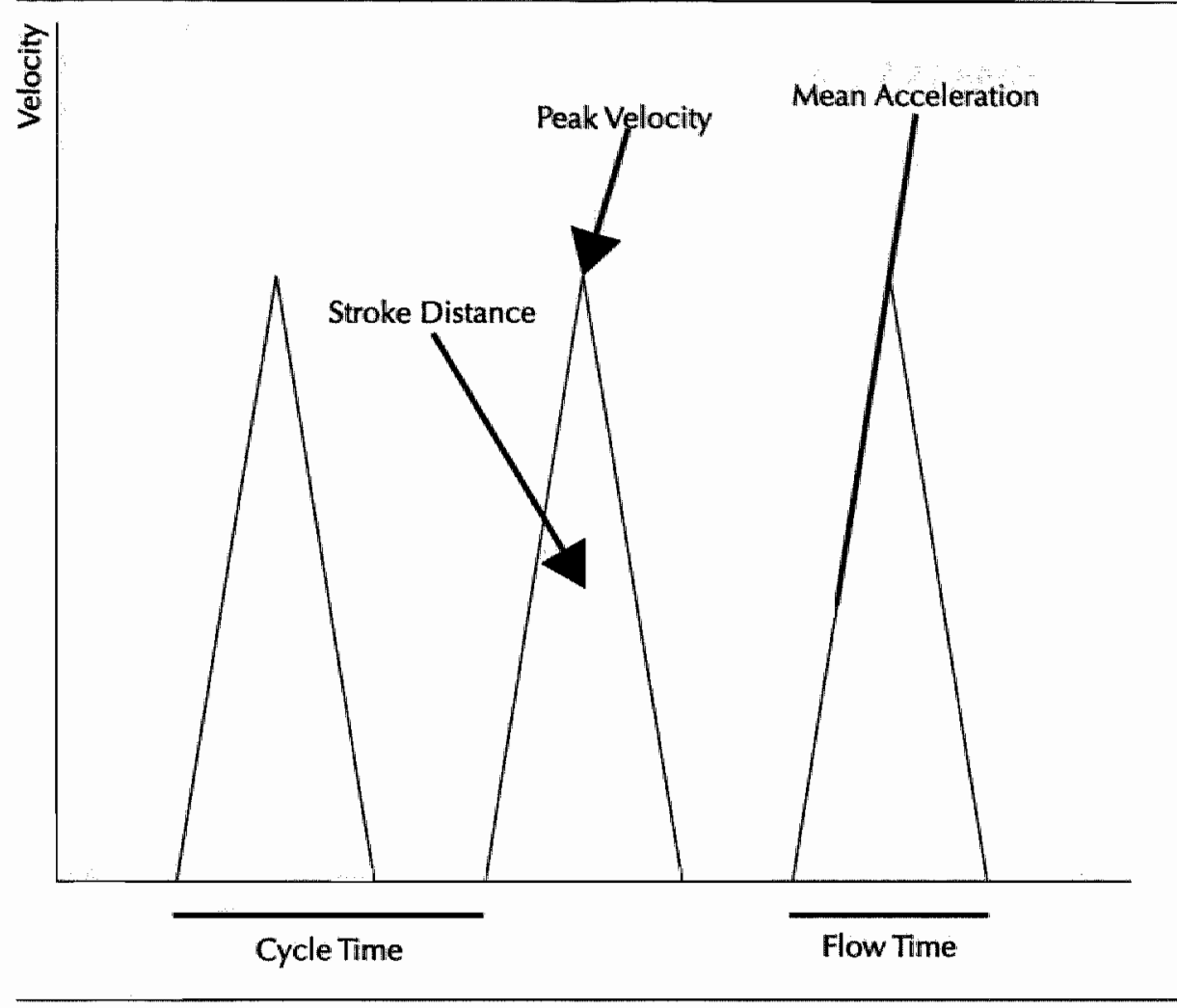

The figure shows a schematic depiction of the back-scattered Doppler frequency shifts producing a typical flow-velocity waveform when aiming at the descending aorta. A number of velocity waveform variables can be measured: stroke distance $(S D)=$ area of the waveform, i.e., the length of a column of blood traversing the aorta with each verticicular stroke; peak velocity $(\mathrm{PV})=$ velocily at the apex of the waveform; mean acceleration $(\mathrm{MA})=$ walue of peak velocity divided by the time needed to reach peak velocity.

Figure 3 Schematic depiction of Doppler flow-velocity waveform

assessed by maximum sound pitch and a sharp, well-defined velocity waveform on the display. Although this technique has the advantage of being totally non-invasive, significant aortic valwe disease will cause turbulence of the blood stream, preventing quantification of the flow. Breathing, movements, a short neck and subcutaneous emphysema are other problems encountered using this approach.

Aortic valve disease, if not too severe, has no influence in the oesophageal approach. The probe can be left in situ for up to two weeks. Studies vallidating Doppler-measured cardiac output against simultaneous thermodilution measurements have found that Doppler values follow changes in cardiac output accurately (18). The tracheal method is a relatively new approach and uses endotracheal tubes with the Doppler probe already incorporated, but the number of studies using this technique has been limited. 
Using the above approach, the following parameters can be measured using transoesophageal Doppler ultrasonography: flow time (FT), peak velocity (PV), ventricular ejection time (VET) and heart rate (HR) (Figure 3). A normogram using and the length, weight and age of the patient can be used to estimate the cross-sectional area (CSA) of the aorta. A newer design combines Doppler flow velocity measurement with $\mathrm{M}$-mode measurement of the aortic diameter. The parameters which can be calculated using these parameters are stroke distance $(S D=V E T . v . d t)$, stroke volume $(S V=S D \times C S A)$, cardiac output $(C O=S V \times H R)$ and corrected flow time (FTC $=F T / V$ cycle time). The stroke distance is the area of the waveform, which is the length a column of blood travels along the aorta with each ventricular stroke. Assuming that during systole CSA changes little during systole, the product of SD and CSA is the volume of blood which is pumped through the vessel with each ventricular stroke. FTc is a correction of flow time for changes in heart rate and provides an indication of alterations in ventricular filling. The peak velocity is the velocity at the apex of the waveform and is an indicator of left ventricular contractility and function.

Recent developments have made $\mathrm{M}$-mode and two-dimensional Doppler echocardiography readily available for continuous use in critically ill patients. One of the advantages is that CSA can be measured during monitoring, which theoretically results in more accurate measurement of the aortic blood flow velocity.

\section{Previous studies}

Most studies of the use of Doppler ultrasonography at the intensive care unit have compared cardiac output measurements obtained using Doppler ultrasonography with cardiac output measurements using thermodilution. Correlation coefficients in these studies varied from 0.58 to 0.98 (mean $=0.86)(18-21)$. One study showed an increase in the correlation coefficient from 0.53 to 0.89 with increasing experience of the examiner, perhaps explaining some of the lower correlation coefficients found (22). Other studies investigated whether Doppler flowmetry could be used for non-invasive optimisation of the left-ventricular filling. One study showed that the ventilatory modus could be optimised, giving patients an optimised level of positive end-expiratory pressure without compromising cardiac performance (23). In a number of studies, the haemodynamic status of surgical patients was optimised during the surgical procedure, in an attempt to reduce morbidity. Mythen et al. showed that intra-operative optimisation of stroke volume was able to reduce post-operative morbidity and the length of hospital stay in cardiac surgery patients (24). These findings were confirmed by Sinclair et al. using the same principle in patients undergoing surgery for a fractured neck of the femur (25). Thus, reversal of intraoperative haemodynamic abnormalities was associated with a reduced morbidity rate. Whether unrecognised haemodynamic deterioration in apparently haemodynamically stable patients could be detected using Doppler ultrasonography in post-operative patients, and what predictive value these findings have, is not known at present. 


\section{Echocardiography}

Echocardiography measures stroke volume (the difference between end-systolic and end-diastolic ventricle volume) to assess cardiac output. Traditionally, it has been based on classical two-dimensional models, using assumptions about the form of the left ventricle. More recently, 3D models have been used to reconstruct the left ventricle. The use of echocardiography at the intensive care unit is often limited by the continuous availability of the echocardiographic module and the lack of the necessary expertise needed to perform this technique.

\section{Bio-impedance}

Stroke volume measurement using the bio-impedance method is based on the principle that blood is a good conductor of electricity and that changes in intrathoracic blood volume during systolic contraction of the left ventricle result in a change in the resistance (impedance) of the chest. However, a considerable variety has been found in the correlation values with thermodilution (26). Moreover, endotracheal tubes, gastric tubes and intravascular catheters, which are frequently used in critically ill patients, seriously disturb the signal.

\section{Regional monitoring level}

\section{Splanchnic blood flow measured by ICG-dilution method}

Technique

In 1945 Bradley introduced splanchnic blood flow estimation based on the Fick principle, using a hepatic venous catheter. In this technique, the right jugular vein is used to insert a $7 F$ cournand catheter into a right hepatic vein under portable fluoroscopy. The correct placement of the catheter tip, in a non-wedged position at least $5 \mathrm{~cm}$ into the vein, is verified using a small amount of contrast dye. Splanchnic blood flow is measured using a primed-continuous indocyanine green (ICG) infusion. A bolus of ICG is followed by a continuous infusion for 30 minutes (12 $\mathrm{mg}$ followed by $1.1 \mathrm{mg} / \mathrm{min}$ ). Splanchnic blood flow is then calculated using the formula:

$Q_{\text {spl }}=\mid R_{I C G} \times \frac{C_{i}}{\left(C_{\text {art }}-C_{h v}\right) \times\left(1-H_{c r}\right)}$

where $I R_{I C G}$ is the ICG infusion rate, $C i$ is the ICG infusate concentration, $C_{\text {art }}$ and $\mathrm{C}_{\mathrm{hv}}$ are the arterial and hepatic venous ICG plasma concentrations and $\mathrm{HCr}$ is the hematocrit value.

Blood samples for ICG concentration determination are taken after 20,25 , and 30 minutes of infusion. The triplicate sampling is performed in order to verify that a steady state level of ICG in blood has been reached. Plasma ICG concentrations are measured spectrophotometrically at a wavelength of $805 \mathrm{~nm}$. 
This splanchnic perfusion measurement is usually performed simultaneously with a measurement of the flow in the leg, as a reference point for the splanchnic blood flow. For this purpose, two additional catheters are inserted, one into the femoral artery and one into the femoral vein. Using the same principle, the difference between the arterial and venous ICGi concentrations is used to calculate the leg flow (27).

\section{Previous studies}

A considerable number of studies have examined total splanchnic blood flow in humans. However, the determinants, clinical course and impact of splanchnic blood flow abnormalities in patients at risk and patients with multiple organ failure have not been clearly established. Patients with an inflammatory response show increased total splanchnic blood flow, measured using the Fick principle, compared to healthy controls (28). At the same time, however, their oxygen consumption increases disproportionately to the increase in splanchnic blood flow. This leads to increased oxygen extraction in the splanchnic area, but there is often a mismatch between oxygen delivery and demand, which is present during hypotension and normotension. Although correction of hypotension increases the blood flow, patient responses are variable (29). Surgery is usually accompanied by an increase in splanchnic blood flow due to the stress response, although low-flow states may also be present in severe hypovolaemia. In low-flow states and hypovolaemia, splanchnic blood flow decreases while the splanchnic metabolic demand shows little change. In these conditions, the above-mentioned redistribution to the splanchnic area is induced to maintain the perfusion of the heart and the brain. Studies using the ICG dilution principle have provided extensive information about these changes during low- and high-flow states. In addition, numerous recent studies have used this technique to evaluate the effects of haemodynamic interventions on the splanchnic blood flow. The use of the inotropes and the vasopressors dopamine, dobutamine, dopexamine, noradrenaline and adrenaline has been extensively evaluated in septic and surgical patients (30).

\section{Gastric and intestinal tonometry}

\section{Technique}

The technique of intestinal tonometry was initially described by Fiddian-Green in the mid-1980s for critically ill trauma patients. A balloon-tipped catheter, the tonometer, is placed in the lumen of the stomach, sigmoid colon or the small intestine (usually during experimental studies). This method allows intraluminal $\mathrm{PCO}_{2}\left(\mathrm{PiCO}_{2}, \mathrm{PgCO}_{2}\right.$ or $\left.\mathrm{PrCO}_{2}\right)$ to be measured, based on the assumption that ischaemia of the splanchnic area is associated with an increased accumulation of $\mathrm{CO}_{2}$ in the intraluminal space and that this $\mathrm{CO}_{2}$ diffuses readily across the gastrointestinal mucosa. Several animal and clinical studies have supported these assumptions (31). Intracellular $\mathrm{CO}_{2}$ depends on both aerobic and anaerobic metabolism. As cellular oxygen supply decreases, the production of $\mathrm{CO}_{2}$ via the aerobic metabolism falls, and below a certain level, the anaerobic metabolism produces lactate and $\mathrm{H}^{+}$ions during the formation of adenosine triphosphate. Buffering of the intracellular $\mathrm{H}^{+}$then leads to $\mathrm{CO}_{2}$ production. This tissue $\mathrm{CO}_{2}$ 
is normally transported to the lungs by venous blood. During hypoperfusion, however, this clearance mechanism is hampered and $\mathrm{CO}_{2}$ will then accumulate in the tissues. $\mathrm{PrCO}_{2}$ therefore depends on metabolism, perfusion and lung physiology (32). Using the measured $\mathrm{PrCO}_{2}$, an intramucosal $\mathrm{pH}(\mathrm{i})$ is calculated using the Henderson-Hasselbalch formula:

$$
\mathrm{pHi}=6.1+\log 10 \times\left(\frac{\text { [bicarbonate } \left._{\text {art }}\right]}{\mathrm{F} \times \mathrm{PrCO}_{2}}\right)
$$

Two techniques for measuring $\mathrm{PrCO}_{2}$ are available clinically, the saline method and the capnographic method, each with their own advantages and disadvantages. In the first method, the tonometer balloon is infused with $2.5-4.5 \mathrm{ml}$ of normal saline or phosphate-buffered saline. After a calibration period of at least 30 minutes, the fluid is withdrawn from the balloon and $\mathrm{PrCO}_{2}$ in this sample is measured with a blood gas analyser. The advantages of this approach are the simple applicability, easy availability, and the lower cost compared to the capnographic method. The disadvantages are that in daily care, practice errors have been observed in $34 \%$ of all measurements (33), related to errors in infused volume and inaccurate timing.

Recently, the introduction of air tonometry, using the Tonocap $(\$$, a modified capnograph, has overcome some of these practical problems. This method uses air as the equilibration medium, which is injected, aspirated and re-injected in a 15-minute cycle. $\mathrm{PCO}_{2}$ in the air from the catheter balloon is measured using the same infrared technology used in capnographs to measure expiratory $\mathrm{PCO}_{2}$. Automated air tonometry has been shown to produce reliable $\mathrm{PrCO}_{2}$ measurements (34). In the first study investigating this method, Creteur et al. found a close correlation between saline-measured $\mathrm{PCO}_{2}$ and gas $\mathrm{PCO}_{2}$ (35). In a study by Graf et al., gastric air tonometry provided an estimation of $\mathrm{PrCO}_{2}$, which was as reliable as saline tonometry, with clinically acceptable precision compared to a predefined $\mathrm{PrCO}_{2}$ (36). In a study by Barry et al., gastric saline and air tonometry were compared in vivo in critically ill patients as well as in vitro with a variation in temperature and carbon dioxide pressures; air tonometry (Tonocap(B) displayed a lower bias in both conditions (37).

Other practical problems are related to the influence of gastric acid secretion on the gastric intramucosal $\mathrm{PrCO}_{2}$. During acid secretion, the $\mathrm{CO}_{2}$ of the mucosa is "buffered" by the $\mathrm{H}+$ of the gastric acid, leading to too low $\mathrm{PCO}_{2}$ values. A number of studies have investigated the use of gastric acid secretion inhibition on tonometric $\mathrm{PrCO}_{2}$, with contradictory results. In a study by Vaisanen et al., the use of randitidine did not alter the $\mathrm{PrCO}_{2}$ measured in critically ill cardiac surgery patients (38). A study by Brinkmann et al., however, found that injection of pentagastrin and omeprazol significantly changed gastric tonometric $\mathrm{pHi}$ in healthy volunteers (39). 


\section{Previous studies}

In recent years, a considerable number of studies using tonometry have been published. Many of these studies focused on the validity and reproducibility of tonometrically derived variables. The validity of gastric intramucosall pHi measurements was assessed by instilling saline directly into the stomach lumen and comparing the intramucosal $\mathrm{pHi}$ with directly measured $\mathrm{pH}$ by inserting electrodes into the stomach wall. The directly and indirectly measured $\mathrm{pH}$ Hi values correlated moderately well.

A number of studies have used tonometry as a predictor of morbidity in surgical and medical patient populations. Roumen et al. found that a low $\mathrm{pHi}$ after major trauma was a predictor of a complicated recovery (40). In particular, patients who never developed a low pHi had an uncomplicated recovery. Fiddian-Green et al. found that in high risk patients undergoing abdominal aortic surgery, a colonic pHi $<6.86$ was associated with the development of ischaemic colitis (41). In another study, this group reported on $103 \mathrm{ICU}$ patients, in whom massive stress ulcer bleeding was associated with a low $\mathrm{pHi}$, whereas patients with $\mathrm{pHi}>7.24$ had no bleeding (42). Interestingly, gastric juice pH was similar in both groups.

Post-operative surgical patients with a pHi below 7.32 at the end of surgery were found to have a significantly longer length of stay in the ICU and the hospital and a greater incidence of major complications (43).

Other studies have investigated the relation between intramucosal $\mathrm{pHi}$ or $\mathrm{pCO}_{2}$ and mortality. In a prospective study, Gutierrez et all. were the first to show that gastric pHi could differentiate surviving critically ill patients from non-surviving patients (44). Marik et al. obtained similar results, concluding that indices of oxygenation, including $\mathrm{pHi}$ and arterial lactate, are superior to haemodynamic or oxygen derived variables as predictors of outcome in patients with sepsis. In a study by Bonham et al., patients with severe pancreatitis with a low intramucosal $\mathrm{pHi}$ had a greater risk of death. An optimal cut-off point for this increased risk was determined at 7.25 (45). In another study, gastric intramucosal pHi was able to identify the critically ill trauma patient who was at high risk of death and multiple organ failure, using a cut-off point of 7.10 (46). Patients who did not correct this initial pHi within 24 hrs were especially prone to developing complications. A study by Miller et al. in a cohort of trauma patients calculated optimal cut-off points for $\mathrm{pHi}$ and gastric-to-arterial $\mathrm{CO}_{2}$ difference with respect to mortality and $\mathrm{MOF}$, using receiver-operator characteristic (ROC) curves (47). Maximised sensitivity and specificity were found at a pHi and $\mathrm{CO}_{2}$ gap of 7.25 and $18 \mathrm{mmHg}$, respectively. 


\section{Other organ-specific tests}

\section{Lactulose/rhamnose intestinal permeability test}

In clinical studies, intestinal permeability is typically measured by monitoring the urinary excretion of one or more enterally administered agents. These agents should be easily measured, should not be normally present in the urine, and should not be degradable or metabolised.

Most permeability tests are based on the conductance of two substances. One agent consists of a relatively small molecule (rhamnose or mannitol), which permeates moderately well through even normal mucosa, while the other consists of a larger molecule (lactulose) and hardly permeates the normal mucosa. Measuring both probes in the urine and assessing their ratio gives an indication of the permeability of the mucosa. It was originally though that the difference in permeability between the two probes was due to differences in the place of transport, i.e., the paracellular (between adjacent cells) and transcellular (primary permeability of epithelium) routes. However, recent data indicate that such a transcellular 'pore' does not exist. Bjarnason et al. found that the difference in permeability may be explained by the surface available for transport across the mucosa (48). Accordingly, smaller molecules can be absorbed through paracellular channels along the entire crypt-villus axis, whereas larger molecules can only be absorbed at the bottom of the villus, between cells lining the crypts of the mucosa. During inflammation, an increased absorption of larger molecules can be caused not only by the widening of tight-junctions but also by a relative increase in the absorptive area for the larger molecules due to villus atrophy. A third mechanism, which was recently suggested, involves the presence of tissue hyperosmolality at the tip of the villus. This tissue hyperosmolality is caused by the countercurrent mechanism in the villus and is increased during disturbed splanchnic mucosal perfusion.

A large number of studies are now available to support the view that intestinal permeability to lactulose and rhamnose is changed in many forms of critical illness, including high-risk surgery, sepsis and MOF. After cardiopulmonary surgery, patients with a gastric $\mathrm{pHi}<7.32$ developed a higher lactulose/rhamnose ratio, while the systemic haemodynamic variables remained stable (49). An increased level of endotoxin in the systemic circulation is associated with increased permeability of the gut (49).

Recent studies have focused on manipulation of the intestinal wall integrity. Sinclair et al. infused dopexamine or dopamine during cardiopulmonary bypass surgery to improve intestinal wall integrity (50). Compared to dopamine, they claimed that dopexamine induced a selective improvement of splanchnic perfusion and reduced intestinal permeability following surgery. In another study, enteral nutrition seemed to improve intestinal permeability in critically ill patients, compared to parenteral nutrition (51).

A recent study by Hallemeesch showed that fluid loading during endotoxaemia increases the lactulose/rhamnose ratio regardless of changes in intestinal permeability (52). These postmucosal factors make the interpretation of changes in individual patients difficult. Moreover, experimental studies have 
revealed increases in gut permeability without changes in endotoxin/microbial translocation (49).

\section{Lidocaine metabolism and MEGX measurements}

One approach to measuring liver function as a part of the liver-gut axis has been to analyse the lidocaine metabolism. Lidocaine, commonly used as local anaesthetic agent, is metabolised by cytochrome P450 $3 \mathrm{~A}$ to form mono-ethylglycinexylidide (MEGX) (49). This de-ethylation of lidocaine is a liver-specific metabolic pathway in the perivenous hepatocytes. Plasma MEGX concentrations can be measured using fluorescence polarisation immunoassay. In clinical practice, a dose of $1 \mathrm{mg} /$ $\mathrm{kg}$ lidocaine is injected intravenously, and the MEGX concentrations are measured in blood samples drawn immediately before and 15 minutes after the lidocaine injection. The amount of lidocaine metabolised is calculated by subtracting the pre-injection MECX concentration from the post-injection MEGX concentration. Because of the relatively high extraction ratio of lidocaine, this liver function not only depends on hepatic metabolic capacity, but also on hepatic blood flow (53). In healthy volunteers, the median MEGX concentration has been established at around $75-100 \mu \mathrm{g} / 1$, and values below $25 \mu \mathrm{g} / \mathrm{l}$ are considered to indicate severely disturbed liver function. This test has predominantly been used in selecting liver transplant patients $(53,54)$, for which lidocaine metabolism seemed to be more useful than ICG metabolism (55). A study in critically ill patients found that the MEGX test seemed to be useful in predicting mortality (56). In this study, MEGX concentrations in non-surviving patients dropped from 20 to $2.4 \mathrm{ng} / \mathrm{ml}$ between admission and day 3 , whereas surviving patients had stable MEGX concentrations. Igonin et al. showed that low MEGX levels were associated with a decreased survival and increased inflammatory response in early sepsis (57).

Other studies, however, have found that low MEGX concentrations did not predict reduced splanchnic blood flow in septic patients, due to the high level of hepatic lidocaine extraction. Moreover, intrahepatic metabolic compartmentalisation may limit the implications of a low lidocaine metabolism.

\section{ICG clearance tests}

Another specific test of liver function involves measuring the clearance of indocyanine green (ICG) from the circulation. This test provides information concerning both the function and perfusion of the liver and can be performed without a hepatic vein catheter. It can be measured using an intravascular catheter or a noninvasive (transcutaneous sensor) method (58). The plasma disappearance rate (PDR) indicates how much (in \%) of the injected ICG initially disappears from the circulating blood per minute due to hepatic elimination, and is calculated as follows:

$\begin{array}{ll}\text { PDR } & =100 \times \ln 2 / t^{1 / 2} \\ \text { with } t^{1 / 2} & =\text { half life of } / C G .\end{array}$


The ICG's primary distribution space is the active circulating blood volume (determined by the total blood volume (TBV)). As liver perfusion is also directly dependent on the blood volume, varying blood volumes will result in different values of PDR even at constant hepatic ICG elimination. Therefore, ICG clearance is best evaluated by multiplying TBV and PDR:

$\begin{aligned} \text { CBig } & =\mathrm{TBV} \times \mathrm{PDR} \\ \mathrm{CPig} & =\mathrm{TBV} \times(1-\mathrm{HCR}) \times \mathrm{PDR} \\ \text { with CBig } & =\text { ICG blood clearance } \\ \mathrm{CPig} & =\text { ICG plasma clearance } \\ \mathrm{HCr} & =\text { haematocrit }\end{aligned}$

ICG is transported to the bile through a membrane transport mechanism which is energy-dependent. The elimination kinetics is dependent on liver perfusion, total circulating blood volume, absorption in the liver cell, intracellular transport and transport into the bile. Normally, more than $50 \%$ of the ICG is extracted by the liver in one passage.

Pollack et al. and Kholoussy et al. found a correlation between ICG clearance and the prognosis for surgical intensive care patients. Patients with a PDR below $6 \%$ did not survive the post-operative period $(59,60)$. In multiple trauma patients, PDR indicated significant liver damage at an early stage (61). Krenn et al. found that short-term PEEP during ventilation in liver transplant patients does not decrease PDR (62).

\section{Lactate metabolism and L- and D-lactate levels}

Lactate measurements in the blood may be used to detect abnormal circulation. Glucose metabolism, which provides a major source of energy, can be viewed as a two-step process. Glucose is first metabolised to pyruvate through a series of anaerobic reactions that yield two molecules of adenosine triphosphate (ATP), i.e., energy. Pyruvate then enters the mitochondria, where it is metabolised to $\mathrm{CO}_{2}$ and $\mathrm{H}_{2} \mathrm{O}$ via the Krebs cycle and the electron transport chain (including the cytochrome chain), generating an additional 34 ATP molecules. Under physiologicall conditions, pyruvate is in equilibrium with lactate and with the oxidised and reduced forms of the cofactor nicotamide adenine dinucleotide $\left(\mathrm{NAD}^{+}\right.$and $\mathrm{NADH}$, respectively), facilitated by lactate dehydrogenase (LDH).

During anoxia, the aerobic or mitochondrial glucose metabolism ceases to function, as a result of which pyruvate accumulates and is converted to lactate (28). Excess lactate formed during these reactions leaves the cell and accumulates in the extracellular fluid. Increased lactate levels in the blood (hyperlactataemia) can result from increased extracellular lactate concentrations when lactate production exceeds lactate utilisation by tissues like the myocardium (which converts lactate into pyruvate) and the liver (which converts it into glucose). 
The most common cause of hyperlactataemia is global tissue hypoxia, which is in most cases due to systemic perfusion abnormalities. Blood lactate measurements can therefore serve as an indicator of impaired tissue oxygenation, although other possible causes must be ruled out. Numerous experimental and clinical studies have correlated increased lactate levels during circulatory failure with poor prognosis (32). Factors which can disturb the interpretation of lactate levels are overproduction or decreased elimination of lactate, the "washout phenomenon', acute changes in acid-base balance, peripheral and visceral tissue perfusion and hepatic lactate uptake.

The presence of hyperlactataemia has been suggested to be a marker of disturbed hepatosplanchnic function, as the gut is a known producer of lactate during sepsis. Under normal conditions, skeletal muscles release lactate, which is largely taken up by the liver (17). The liver has a capacity to increase lactate uptake during non-physiological conditions. Lactate production by individual organs is probably a more reliable index of anaerobic metabolism than simple arterial lactate concentration. Lactate flux can be calculated from measurements of the organ blood flow and the arterial-venous lactate difference. However, individual organ production is difficult to measure in the clinical setting. Measurements of hepatic vein lactate concentration have been used to assess splanchnic lactate metabolism, since the liver has a major impact on blood lactate concentrations. Landow et al. found a correlation between hepatic vein lactate concentrations and gastric intramucosal pHi in cardiac surgery patients (63). Decreased lactate extraction was associated with gastric intramucosal acidosis and increased systemic and hepatic venous lactate concentrations. However, Takala et al. showed that this relationship between hepatic venous lactate concentrations and $\mathrm{pHi}$ is not so obvious for individual patients (64). The increased lactate concentrations in the hepatic vein are not likely to be correlated with decreased lactate uptake by the liver but are correlated with increased splanchnic lactate production. Moreover, the routine insertion of a hepatic vein catheter may impose significant risk on the patient, through increased risks of infections and emboli.

Tenhunen et al. have suggested measuring intestinal intraluminal lactate to gain a better idea of the regional lactate metabolism (65). In their study, luminal mesenteric lactate increased during cardiac tamponade-induced reduced aortic flow. No human data is as yet available. Others have suggested measuring the isomere D-lactate as a marker of splanchnic hypoperfusion. D-lactic acidosis in patients has originally been described for the short bowel syndrome $(66,67)$, and is caused by the production of D-lactic acid by bacterial fermentation in the gut lumen and its subsequent absorption into the blood. Klebsiella, Escherichia coli, Lactobacillus acidophilus and Bacteroides species are known to be capable of synthesizing D-lactate (68). Although both L-lactate and D-lactate are produced, only the D-isomere accumulates, due to the rapid metabolism of L-lactate in the liver. Moreover, although D-lactate dehydrogenase is present in certain lower animal species, only L-lactate dehydrogenase is present in humans. D-lactate cannot be metabolised by humans, and is excreted in the urine (69). The site of D-lactic acid production is likely to be the colon, since colonic bacteria in humans 
metabolise carbohydrates that have not been metabolised in the small intestine.

During intestinal ischaemia, the resident microbial flora of the intestine multiplies rapidly in the ischaemic segment $(68)$. This bacterial proliferation occurs in conjunction with disruption of the mucosal layer of the gut. Both experimental and clinical studies have shown that acute intestinal ischaemia causes a rise in D-lactate levels in the systemic circulation $(68,69)$. In a study by Murray, increased D-lactate levels in the blood were shown to be able to differentiate with high precision and validity between mesenteric ischaemia and other (non-ischaemic) abdominal catastrophes (69).

\section{Regional amino acid metabolism measurements with special reference to nitric oxide metabolism}

During sepsis and organ failure, major changes occur in whole body and organ metabolism. The major feature is increased nitrogen loss as 'catabolic' loss. The body's response to sepsis and organ failure is an increase in both synthesis and breakdown of proteins, with breakdown predominating. Skeletal muscle proteins are broken down in order to provide amino acids for alternative protein synthesis, for example the acute phase proteins which are synthesized by the liver in large quantities during inflammation.

Stable and radioactive isotopes of amino acids have been used for the measurement of protein turnover and amino acid flux. Whole body protein turnover can be calculated using a one-compartment model, which evaluates the exchange of amino acids from and into the extracellular free amino acid pool. The model involves administering a primed constant infusion of labelled amino acid until a steady state of the ratio between the tracer and tracee (Tracer-Tracee Ratio $=$ TTR) is reached within the plasma pool, after which samples are drawn from this plasma pool. At steady state, the amount of tracer amino acid lost will be equal to the tracer amino acid infused, and the plasma Ra (rate of appearance in $\mathrm{nmol} / \mathrm{min}$ ) is calculated using the following equations:

(1) $\mathrm{Ra}$

$\begin{array}{lll}\mathrm{Ra}= & \mathrm{I} / \mathrm{TR}_{\mathrm{A}} \\ \text { where, } & \mathrm{Ra}= & \\ & \mathrm{I}= & \mathrm{Rd} \\ & \mathrm{Ra} \times T T R_{\mathrm{A}}\end{array}$

In these equations Rd is the rate of disappearance in $\mathrm{nmol} / \mathrm{min}, 1$ the infusion rate of the labelled amino acid, and $\mathrm{TTR}_{\mathrm{A}}$ the tracer-tracee ratio of the labelled amino acid at steady state. The whole body substrate flux is defined as Ra ( $\mathrm{nmol} / \mathrm{kg}$ body weight/min).

Organ flux measurements can be made using a two compartment model, in which two catheters are placed, one in the afferent blood vessel and one in the efferent blood vessel of the organ, allowing concentration differences to be measured across this organ. However, measuring concentrations of the amino acids only yields information on the net uptake and release, not on dynamic processes, such 
as disposal and production. The use of labelled amino acids (tracers) enables measurements of amino acid disposal and production across an organ. The abovementioned protocol with primed constant infusion can be used to measure the arterial and venous TTR and calculate protein degradation. The TTR of the amino acid is measured by means of a high performance liquid chromatography (HPLC) method coupled with a mass spectrometry (MS) system (LCQ, Finnigan) (70). TTR values are obtained by subtracting pre-tracer infusion TTR values.

Stable isotopes can be used to determine in-vivo whole-body and hepatosplanchnic synthesis rates. The whole-body rates of appearance of L-arginine $\left(R_{A R C}\right)$ and citrulline $\left(\mathrm{Ra}_{\mathrm{ClT}}\right)$ are derived from formula (1) using the $\mathrm{N}_{2}$-arginine and the $C_{1} D_{2}$-citrulline isotope, respectively.

The endogenous rate of $\mathrm{Ra}_{\mathrm{ARO}}$, which is assumed to reflect de-novo $\mathrm{L}$-arginine synthesis plus $L$-arginine appearance derived from protein breakdown, can be estimated by correcting the total $\mathrm{Ra}_{\mathrm{ARG}}$ for the contribution of the L-arginine that was infused. Formula (2) is used to calculate the rate of whole-bady synthesis of $\mathrm{NO}\left(\mathrm{Ra}_{\mathrm{NO}}\right)$ based upon the plasma isotope transfer from $\mathrm{N}_{2}$-arginine to $N_{1}$-citrulline.

(2) $\mathrm{Ra}_{\mathrm{NO}}=\mathrm{Ra}_{\mathrm{CIT}} \times\left(\mathrm{TTR}_{\mathrm{AN1}}-\mathrm{CIT} / \pi T R_{\mathrm{AN} \text { N-ARC }}\right)$

$T_{T R}$ N1-CIT and TTR ${ }_{A}$ N2-ARG represent the TTR values of $N_{1}$-citrulline and $\mathrm{N}_{2}$-arginine in arterial plasma, respectively.

For practical purposes, the portal-drained viscera (PDV) are defined as the total of all portal-drained organs which, besides the spleen, stomach and pancreas, largely represent the intestines. The splanchnic area includes the the portal-drained viscera and the liver, so that liver values can be obtained by subtracting portal-drained viscera from splanchnic values. L-arginine and NO metabolism across the portal-drained viscera, the splanchnic region and the liver can be calculated in a two-compartment model.

The rate of $\mathrm{NO}$ synthesis by individual organs $\left(\mathrm{Syn}_{\mathrm{NO}}\right)$ can be calculated from the conversion of $\mathrm{N}_{2}$-arginine to $\mathrm{N}_{1}$-citrulline across the organ. The term $\mathrm{nb} \mathrm{N}_{\mathrm{N} \text { - } \mathrm{CT}}$ represents the tracer net balance of the $N_{1}$-citrulline isotope in arterial and venous plasma, respectively. Because of possible fractional extraction of citrulline and hence $N_{1}$ citrulline $\left(\mathrm{FE}_{\mathrm{CIT}}\right)$ by the organ, the $\mathrm{nb}_{\mathrm{N} 1-\mathrm{CIT}}$ must be corrected for the loss of $N_{1}$-citrulline tracer across the organ, which is possible by using the $C_{1} D_{2}$-citrulline isotope in equation (3). The term $(1-F E)$ represents the fraction of total amino acid that bypasses metabolism in the organ and so appears in the output; this is used to correct the $N_{1}$-citrulline nb (4). The organ Syn No is derived from formula (5), in which $T T R_{A}$ N2-ARG represents arterial $\mathrm{N}_{2}$-arginine enrichment. The TTR of $\mathrm{N}_{2}$-arginine in the arterial plasma-free pool is assumed to be the same as the TTR of $\mathrm{N}_{2}$-arginine in the direct precursor pool of NO synthesis.

(3) $\left.\mathrm{FE}_{\mathrm{CIT}}=\left(\mathrm{TTR}_{\mathrm{A}} \times[\mathrm{A}]-\mathrm{TTR}_{\mathrm{V}} \times[\mathrm{V}]\right) / T \mathrm{TR}_{\mathrm{A}} \times[\mathrm{A}]\right)_{\mathrm{CID} 2 \mathrm{CIT}}$

(4) $\mathrm{nb}_{\mathrm{N1}-\mathrm{CIT}}=$ flow $\times\left\{[\mathrm{V}] \times T \mathrm{TR}_{\mathrm{V}}-[\mathrm{A}] \times T \mathrm{R}_{\mathrm{A}} \times\left(1-\mathrm{FE}_{\mathrm{CIT}}\right)\right\}_{\mathrm{N} 1-\mathrm{CIT}}$

(5) Syn $_{N O}=n b_{N 1-G I T} / T T R_{A N 2-A R G}$ 


\section{REFERENCES}

1. Vincent IL, Thijs L, Cerny V. Critical care in Europe. Crit Care Med 1997;13:245-254.

2. Rosenwasser $\mathrm{RH}$, Jallo JL, Gretch $\mathrm{CC}$, et all. Complications of Swan-Ganz catheterization for hemodynamic monitoring in patients with subarachnoid hemornage. Neurosurgery 1995;37: 872-875.

3. Putterman CE. The Swan-Ganz catheter: a decade of hemodynamic monitoring. I Crit Care 1989:4:127.

4. Shah KB, Rao TLK, Laughlin $S$, et al. A review of pulmonary artery catheterization in 6,245 patients. Anesthesiology 1984;61:271-275.

5. Connors AF, Speroff T, Dawson NV, et al. The effectiveness of right heart catheterization in the initial care of critically ill patients. JAMA 1996;276:889-897.

6. Boyd O, Hayes M. The ocygen trial: the goal. Br Med Bull 1999;55:125-139.

7. Shoemaker WC, Appel PL, Kram HB. Measurement of tissue perfusion by oxygen transport patterns in experimental shock and in high-risk surgical patients. Intensive Care Med 1990;16(Suppl 2):S135-\$144

8. Shoemaker WC, Appel PL, Kram HB, Waxman K, Lee TS. Prospective trial of supranormal values of survivors as therapeutic goals in high-risk surgical patients. Chest 1988;94(6):1176-86.

9. Ince $C$, van der Sluijs JP, Sinaasappel $M$, Avontuur JA, Coremans $J M$, Bruining HA. Intestinal ischemia during hypoxia and experimental sepsis as observed by $\mathrm{NADH}$ videofluorimetry and quenching of Pd-porphine phosphorescence. Adv Exp Med Biol 1994;361:105-10.

10. Sanchez-Garcia $M$, Cambronero IA, Lopez J, et al. Reduced incidence of nosocomial pneumonia and shorter ICU stay in intubated patients with the use of selective decontamination of the digestive tract (SDD): a multicenter, double blind, placebo-controlled study [Abstract] Intensive Care Med 1992;

11. Palomar $M$, Alvarez $F$, Jorda $R$, et al. Nosocomial pnuemonia: selective digestive decontamination and sucralfate. [Abstract] Intensive Care Med 1992; S13.

12. Laggner $A N$, Tryba $M$, Georgopoulos $A$, et al. Oropharyngeal decontamination with gentamicin for long-term ventilated patients on stress ulcer prophylaxis with sucralfate? Wien Klin Wochenschr 1994;106(1):15-9.

13. Kisch $H$, Leucht $S$, Lichtwarck-Aschoff $M_{r}$ Pfeiffer UJ. Accuracy and reproducibility of the measurement of actively circulating blood volume with an integrated fiberoptic monitoring system. Crit Care Med 1995;23(5):885-93.

14. Lichtwarck-Aschoff $M$, Beale R, Pfeiffer UJ. Central wenous pressure, pulmonary artery occlusion pressure, intrathoracic blood volume, and right ventricular end-diastolic volume as indicators of cardiac preload. J Crit Care 1996; 11(4):180-8.

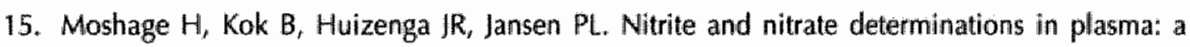
critical evaluation. Clin Chem 1995;41(6 Pt 1):892-6.

16. Puyana IC, Soller BR, Zhang $\mathrm{S}$, Heard SO. Continuous measurement of gut pH with nearinfrared spectroscopy during hemorrhagic shock. J Trauma 1999;46(1):9-15.

17. Mitchell JP, Schuller D, Calandrino FS, Schuster DP. Improved outcome based on fluid management in critically ill patients requiring pulmonary artery catheterization. Am J Respir Crit Care Med 1992;145:990-8.

18. Davies IN, Allen DR, Chant ADB. Non-invasive Doppler-derived cardiac output: a validation study comparing this technique with thermodilution and Fick methods. Eur J Vasc Surg 1991;5:497-500. 
19. Gardin JM, Dabestani A, Matin K, Allfie A, Russell D, Henry WL. Reproducibility of Doppler aortic blood flow measurments studies on intraobserver, interobserver and day-to-day variability in nomal subjects. Am / Cardiol 1984,54:1092-8.

20. Ihlen $H_{*}$ Endresen $K$, Golf $S_{n}$ Nitter-Hauge $S$. Cardiac stroke volume during exercise measured by Doppler echocardiography: comparison with the thermodilution technique and evaluation of reproducibility. Br Heart j 1987;58:455-9.

21. Krishnamurthy B, MCMurray T), McClean E. The peri-operative use of the oesophageal Doppler monitor in patients undergoing coronary artery revascularisation. A comparison with the continuous cardiac output monitor. Anaesthesia 1997;52:624-9.

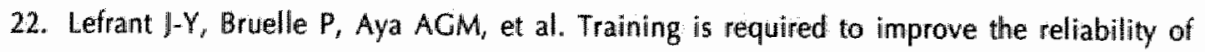
esophageal Doppler to measure cardiac output in critically ill patients. Intensive Care Med $1998 ; 24: 347-52$.

23. Singer $M_{r}$ Bennett $D$. Optimisation of positive and expiratory pressure for maximal delivery of oxygen to tissues using oesophageal Doppler ultrasonography. BM) 1989;298(6684):1350-3.

24. Mythen $M G$, Webb AR. Perioperative plasma volume expansion reduces the incidence of gut mucosal hypoperfusion during cardiac surgery. Arch Surg 1995;130(4):423-9.

25. Dhainaut $\mathbb{H} F$, Tenaillon $A$, Hemmer $M$, et al. Confirmatory platelet-activating factor receptor antagonist trial in patients with severe gram-negative bacterial sepsis: a phase III, randomized, double-blind, placebo-controlled, multicenter trial. BN 52021 Sepsis Investigator Group. Crit Care Med 1998;26(12):1963-71.

26. Pedoto A, Tassiopoulos AK, Oler $A$, et al. Treatment of septic shock in rats with nitric oxide synthase inhibitors and inhaled nitric oxide. Crit Care Med 1998;26(12):2021-8.

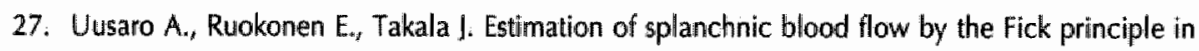
man and problems in the use of indocyanine green. Carciovasc Res 1995;30:106-12.

28. Takala J, KKinney JM, Tucker HN (editors). Organ Metabolism and Nutrition: Ideas or Future Critical Care. led. New York: Raven Press Ltd.; 1994; 18, Sepsis and human splanchnic metabolism, p. 369-79.

29. Takala I. Determinants of splanchnic blood flow. Br J Anaesth 1996;77:50-8.

30. Saetre T, Gundersen $Y$, Smiseth $O A$, ef al. Hepatic oxygen metabolism in porcine endotoxemia: the effect of nitric oxide synthase inhibition. Am ) Physiol 1998;275(6 Pt 1):G1377-G1385

31. Pastores SM, Katz DP, Kvetan V. Splanchnic ischemia and gut mucosal injury in sepsis and the multiple organ dysfunction syndrome. Am J Gastroenterol 1996:91(9):1697-710.

32. Anonymous. Third European Consensus Conference in Intensive Care Medicine. Tissue hypoxia. How to detect, how to correct, how to prevent? Am / Respir Crit Care Med 1996;154(5):1573-8.

33. Brinkert W, Bakker J. Is it time to abandon the pHi concept? Int I Intens Care 1998;16-21.

34. Heinonen $\mathrm{PO}$, Jousella IT, Blomqvist KA, Olkkola KT, Takkunen. Validation of air tonometric measurement of gastric regional concentrations of $\mathrm{CO}_{2}$ in critically ill septic patients. Intensive Care Med 1997;23:524-9.

35. Shoemaker WC, Belzberg $\mathrm{H}$, Wo $\mathrm{CC}_{\text {, }}$ et al. Multicenter study of noninwasive monitoring systems as alternatives to imwasive monitoring of acutely ill emergency patients. Chest 1998;114(6):1643-52.

36. Billiar $T R$, Langrehr $J M$, Curran $R D$, et al. Two unique aspects of inducible. $N=O$ synthase in liver cells and accessory cells: hepatic damage is minimized by hepatocyte $. N=O$ production and immunoregulation is mediated by macrophage. $\mathrm{N}=\mathrm{O}$ production. Res Immunol $1991 ; 142(7): 584-6$.

37. Ochoa JB, Udekwu AO, Billiar $T R$, et al. Nitrogen oxide levels in patients after trauma and during sepsis. Ann Surg 1991;214(5):621-6. 
38. Sullivan BJ, Swallow CJ, Girotti MJ, Rotstein OD. Bacterial translocation induces procoagulant activity in tissue macrophages. A potential mechanism for end-organ dysfunction. Arch Surg $1991 ; 126(5): 586-90$.

39. Southan GJ, Szabo C, Connor MP, Salzman AL, Thiemermann C. Amidines are potent inhibitors of nitric oxide synthases: preferential inhibition of the inducible isoform. Eur J Pharmacol 1995; 291(3):311-8.

40. Southan G], Szabo $C_{r}$ Thiemermann $C$. Isothioureas: potent inhibitors of nitric oxide synthases with variable isoform selectivity. Br I Pharmacol 1995;114(2):510-6.

41. Schiedler $M G$, Cutler $\mathbb{N S}$, Fiddian Green RC. Sigmoid intramural $\mathrm{pH}$ for prediction of ischemic colitis during aortic surgery: a comparison with risk factors and inferior mesenteric artery stumo pressures. Arch Surg 1987;122:881-6.

42. Fukuto $\mathrm{J}$, Chaudhuri $G$. Inhibition of constitutive and inducible nitric oxide synthase: potential selective inhibition. Annu Rev Pharmacol Toxicol. 1995;35:165-94.

43. Mythen $M$, Webb $A R$. Intra-operative gut mucosal hypoperfusion is associated with increased post-operative complications and cost. Intensive Care Med 1994;20:99-104.

44. Gutierrez $G$, Palizas $F$, Doglio $G$, et al.. Gastric intramucosal $\mathrm{pH}$ as a therapeutic index of tissue oxygenation in critically ill. Lancet 1992;339(1):195-9.

45. Robertson FM, Offner PJ, Ciceri DP, Becker WK, Pruitt-BA J. Detrimental hemodynamic effects of nitric oxide synthase inhibition in septic shock. Arch Surg 1994;129(2):149-55.

46. Chang MC, Cheatham ML, Nelson LD, Rutherford E), Morris-JA J. Castric tonometry supplements. information provided by systemic indicators of oxygen transport. J Trauma 1994;37(3):488-94.

47. Lefer AM. Induction of tissue injury and altered cardiovascular performance by plateletactivating factor: Relevance to multiple system organ failure. Crit Care Clin 1989;5:331-5.

48. Minnard EA, Shou I, Naama $H$, Cech A, Gallagher $H$, Daly JM. Inhibition of nitric oxide synthesis is detrimental during endotoxemia. Arch Surg 1994;129(2):142-7.

49. Brinkmann $A$, Calzia $E_{r}$ Träger $K$, Radermacher $P$. Monitoring the hepato-splanchnic regiona in the critically ill patient. Measurement techniques and clinical relevance. Intensive Care Med 1998;24:542-56.

50. Sinclair DG, Houldsworth PE, Keogh B, Pepper J, Evans TW. Gastrointestinal permeability following cardiopulmonary bypass: a randomised study comparing the effects of dopamine and dopexamine. Intensive Care Med 1997;23:310-6.

51. Szabo $C$, Southan $G$ ), Thiemermann $C$. Beneficial effects and improved survival in rodent models of septic shock with S-methylisothiourea sulfate, a potent and selective inhibitor of inducible nitric oxide synthase. Proc Natl Acad Sci USA 1994:91(26):12472-6.

52. Chartrain NA, Geller DA, Koty PP, et al. Molecular cloning, structure, and chromosomal localization of the human inducible nitric oxide synthase gene. J Biol Chem 1994;269(9):6765-72.

53. Oellerich $M$, Ringe $B$, Gubernatis $G_{r}$ et al. Lignocaine metabollite formation as a measure of pretransplant liver function. Lancet 1989;25:640-2.

54. Schinella M, Guglielmi A, Veraldi GF, Boni M, Frameglia M, Caputo M. Evaluation of the liver function of cirrhotic patients based on the formation of monoethylglycine Xylidide (MEGX) from lidocaine. Eur J Clin Chem Clin Biochem 1994;31:553-7.

55. Oda $Y$, Kariya $N$, Nakamoto T, Nishi S, Asada A, Fujimori $M$. The monoethylglycinexylidide test is more useful for evaluating liwer function than indocyanine green test: case of a patient with remarkably decreased indocyanine green half-life. Ther Drug Monit 1995;17:207-10.

56. Maynard ND, Bihari DJ, Dalton RN, Beale R, Smithies MN, Mason RC. Liver function and splanchnic ischemia in critically ill patients. Chest 1997;111(1):180-7. 
57. Celler $\mathrm{DA}$, Freeswick $\mathrm{PD}, \mathrm{Nguyen} \mathrm{D}$, et al. Differential induction of nitric oxide synthase in heppatocytes during endotoxemia and the acute-phase response. Arch Surg 1994:129(2);165-71.

58. Freeswick PD, Wan $Y$, Celler DA, Nussler AK, Billiar TR. Remote tissue injury primes hepatocytes for nitric oxide synthesis. I Surg Res 1994:57(1):205-9.

59. Kholoussy AM, Pollack D, Matsumoto T. Prognostic significance of indocyanine green clearance in critically ill surgical patients. Crit Care Med 1984;12(2):115-6.

60. Pollack DS, Sufian S, Matsumoto T. Indocyanine green clearance in critically ill patients. Surg Cynecol Obstet 1979;149:853-4.

61. Cottlieb ME; Stratton HH, Newell JC, Shah DM. Indocyanine green. Its use as an early indicator of hepatic dysfunction following injury in man. Arch Surg 1984;119:264-8.

62. Older $P$, Smith $R$, Courtney $P$, Hone $R$. Preoperative evaluation of cardiac failure and ischemia in elderly patients by cardiopulmonary exercise testing. Chest 1993;104(3):701-4.

63. Jacob TD, Ochoa JB, Udekwu AO, et al. Nitric oxide production is inhibited in trauma patients. J Trauma 1993;35(4);590-6.

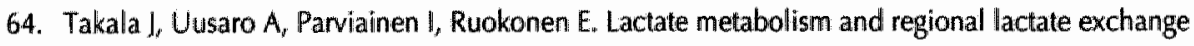
after cardiac surgery. New Horiz 1996;4(4):483-91.

65. Tenhunen J), Jakob $\mathrm{S}$, Ruokonen $\mathrm{E}$, Takalla J. Jejunal luminal microdialysate lactate in cardiac tamponade - effect of low systemic blood flow on gut mucosa. Intensive Care Med 2002;28(7): 953-962.

66. Oh MS, Phelps KR, Traube M, Barbosa-Saldivar IL, Boxhill $C_{r}$ Carroll HJ. D-lactic acidosis in a man with the short-bowel syndrome. New Eng J Med 1979;301(5):249-52.

67. Hove $\mathrm{H}$, Mortensen PB. Colonic lactate metabolism and D-lactic acidosis. Dig Dis Sci 1995;40(2):320-30.

68. Murray MI, Barbose II, Cobb CF. Serum D(-)-lactate levels as a predictor of acute intestinal ischemia in a rat model. I Surg Res 1993;54(4):507-9.

69. Murray MI, Gonze MD, Nowak LR, Cobb CF. Serum D(-)-lactate levels as an aid to diagnosing acute intestinal ischemia. Am J Surg 1994;167(5):575-8.

70. van $\mathbb{E}$ ijk HM, Rooyakkers DR, Deutz NEP. Rapid routine determination of amino acids in plasma by high-performance liquid chromatography with a 2-3 microns Spherisorb ODS II column. I Chromatogr 1993;620(1):143-8. 
CHAPTER 3

Outline of thesis 
The main hypothesis of this thesis is that dysfunction of the hepatic and splanchnic organs plays an important role in the pathophysiology of multiple organ failure and sepsis in humans. The first two chapters have outlined the importance of disturbances in the functioning of these organs for the development and continuation of multiple organ failure. The aim of the research underlying this thesis was to evaluate two aspects of this hypothesis. The first is the idea that monitoring splanchnic perfusion is a better predictor of the outcome for the septic patient at the intensive care unit than the use of routinely used measurements of systemic perfusion. This also implies that patients can be equally effectively monitored peri-operatively using global monitoring techniques. The second aspect is the idea that modulation of the splanchnic function (i.e. perfusion and metabolism) may be able to influence the morbidity and mortality from multiple organ failure.

The studies described here emphasised the difference between global and regional perfusion as described in the previous chapter. In addition, the difference between surgical patients and critically ill septic patients in terms of using monitoring and optimising techniques at the intensive care unit was considered to be an important determinant in reviewing the results.

Chapter 4 evaluates the use of transoesophageal Doppler ultrasound in monitoring global perfusion in surgical patients. The chapter tries to establish whether measurement of global perfusion is predictive of the development of serious post-operative complications. Various aspects of intra-operative and post-operative monitoring and optimisation of the global perfusion are discussed.

Chapters 5, 6 and 7 discuss the monitoring and optimisation of global perfusion in critically ill patients. Chapter 5 discusses the use of dopexamine in high-risk surgical patients to decrease mortality. Chapter 6 reviews several studies which have evaluated the influence of optimising global perfusion on outcome. One of our other questions was whether in critically ill, haemodynamically unstable patients, regional parameters are better monitoring parameters of intensive care resuscitation than global haemodynamic parameters. This question is addressed in chapter 7.

Chapters 8 and 9 discuss studies on the use of regional parameters of disturbed organ function in surgical patients to assess the role of splanchnic dysfunction in the development of organ failure. The study reported on in chapter 8 aimed to establish whether D-llactate could be used as a predictor of the occurrence of gut ischaemia after high-risk surgery. The relationship between D-lactate and splanchnic perfusion, indicated by intramucosal $\mathrm{PrCO}_{2}$ and $\mathrm{pHi}$, is dicussed in chapter 9.

The use of tonometry as a marker of splanchnic hypoperfusion in pre-operative outcome prediction is the subject of chapter 10, while chapter 11 reviews the relation between splanchnic perfusion and the inflammatory response in this region. This chapter discusses whether such a relationship could be detected soon after the surgical procedure. 
Chapters 12 and 13 report on research investigating whether it would be possible to modulate splanchnic perfusion and outcome in an animal experimental model, using either inhibition (chapter 12) or stimulation (chapter 13) of nitric oxide production.

In summary, the main questions addressed in this thesis are:

1. Does monitoring the global haemodynamic status of high-risk surgical patients using transoesophageal Doppler ultrasonography improve the predictability of postoperative morbidity? (Chapter 4)

2. Does improvement of the global haemodynamic parameters using fixed doses of inotropics reduce mortality and morbidity in high-risk surgical patients? (Chapter 5)

3. Does improvement of the global haemodynamic parameters improve outcome in high-risk surgical patients and does this treatment improve outcome in septic patients? (Chapter 6)

4. Does monitoring regional perfusion improve the predictability of morbidity more than global haemodynamic parameters in critically ill patients? (Chapter 7)

5. Is the measurement of D-lactate as a regional parameter suitable for the detection of intestinal ischaemia in high-risk surgical patients? (Chapter 8 )

6 . Is a decreased regional oxygenation of the intestinal mucosa associated with an increased D-lactate level in critically ill patients? (Chapter 9)

7. Does pre-operatively decreased regional oxygenation predict post-operative outcome in high-risk surgical patients? (Chapter 10)

8. Does an increased inflammatory response in the hepatosplanchnic area precede the development of organ failure in high-risk surgical patients? (Chapter 11)

9. Does reduction of the inflammatory response by inhibiting nitric oxide production improve the perfusion and functioning of the hepatosplanchnic area in experimental sepsis and organ failure? (Chapter 12)

10. Does increasing nitric oxide production improve the perfusion and functioning of the hepatosplanchmic area in experimental sepsis and organ failure? (Chapter 13) 
CHAPTER 4

Prediction of postoperative cardiac surgical morbidity and organ failure within 4 hours of icu admission using oesophageal doppler ultrasonography

\section{Published in}

Crit Care Med, 1999; 127(7):1288-1294. 


\section{ABSTRACT}

Objective, To compare oesophageal Doppler ultrasonography with standard haemodynamic variables used in postoperative care for the prediction of postoperative complications after cardiac surgery.

Design: Prospective, observational pilot study.

Setting. University Hospital multidisciplinary intensive care unit (ICU).

Patients: Postoperative cardiac surgical patients.

Interventions. Standard postoperative management as guided by routinely measured haemodynamic variables.

Measurements: An oesophageal Doppler ultrasound probe was inserted for measurement of stroke volume (SV), cardiac output (CO), and other flow-related variables. Both these and routine haemodynamic variables (mean arterial pressure (MAP), central venous pressure (CVP), heart rate, arterial base deficit, urine output, core-toe temperature difference) were recorded at half-hourly intervals for the first four postoperative hours. The incidence of systemic inflammatory response syndrome (SIRS) at 24hrs, Acute Physiology and Chronic Health Evaluation (APACHE) 11, and multiple organ failure (MOF) scores, postoperative complications, and length of ICU and hospital stays were recorded.

Main results: Twenty consecutively admitted patients were studied; eight after emergency bypass grafting and twelve following elective bypass grafts and/or valve replacement. Nine patients developed post-operative complications of whom two died. At admission, significant differences were seen between complicated and uncomplicated patients for SV, heart rate, and standard base excess, but not for CO. By using receiver operator characteristic curves, SV was the best marker for predicting postoperative complications during the initial postoperative period.

Conclusions: A low SV and high heart rate, both at ICU admission and during the subsequent four hours were the best prognostic factors for development of complications following cardiac surgery. Cardiac output values were not useful. This pilot study suggests that the minimally invasive technique of oesophageal Doppler ultrasonography may be a useful tool to assist early prognostication. 


\section{INTRODUCTION}

In recent years there has been an increasing understanding of the pathophysiology of complications in post-traumatic and post-operative intensive care unit (ICU) patients. Shock is now defined, regardless of the underlying cause, as an acute imbalance between supply and demand of oxygen, in which inadequate perfusion or tissue dysoxia lead to cellular damage and, ultimately, dysfunction of major organ systems (1). Although the process of shock can be defined in this way, the problem remains in identifying the patient in shock, especially in the early stages $(2,3)$. This is hampered, by the compensatory mechanisms through which the patient remains normotensive and normoxaemic. Perfusion may be inadequate from an early stage and may lead to activation of inflammatory pathways (4). By definition, such patients will exhibit a systemic inflammatory response syndrome (SIRS) and be susceptible to the development of subsequent complications (5).

Although only a small proportion of post-operative patients die, significant postoperative morbidity with subsequent prolongation of ICU stay underlines the importance of early recognition and correction of the inadequately resuscitated patient. Thermodilution cardiac output measurement has been traditionally used for this purpose (6). However, this usually requires pulmonary artery catheterisation, with its accompanying complications, such as thrombosis (0-1.4\%) (7), arrhythmias (1.3-1.5\%) (8), pneumothorax (1\%) (9) and catheter related-sepsis $(1 \%)(10)$, in addition to additional time, effort and expertise. The retrospective study by Connors et al. also suggested significant increases in mortality through its use via mechanisms as yet undefined (11).

Studies have shown that oesophageal Doppler ultrasonography is a well-validated, minimally invasive method for monitoring cardiac output in critically ill intubated patients (12-14). Examination of the systolic flow velocity waveforms, produced by analysis of Doppler frequency shifts, yields additional information related to cardiac performance and intravascular volume status $(15,16)$. We therefore decided to investigate its prognostic utility in an observational study monitoring the immediate postoperative course following cardiac surgery

\section{MATERIALS AND METHODS}

Approval to undertake this study was granted by the Hospital Ethical Committee. Informed patient consent was obtained preoperatively. A Parsonnet score was calculated to prestratify cardiac surgical patients for their risk of developing postoperative complications $(17,18)$. Those returning with gross hemodynamic instability, i.e. requiring aortic balloon counterpulsation and/or large inotrope doses were excluded from the study because such patients are highly likely to develop complications and the study aim was to identify the not-so-obvious at-risk patient. 
An Acute Physiology and Chronic Health Evaluation (APACHE) II score was recorded on ICU admission and over the first $24 \mathrm{hrs}$. Criteria needed to define systemic inflammatory response syndrome (SIRS) were also recorded the first $24 \mathrm{hrs}$ (19).

Patients were monitored during the postoperative period with standard (routine) haemodynamic variables, using arterial and central venous catheters. Measurement of blood pressure (MAP), heart rate (HR), and central venous pressure (CVP) were recorded at least every $30 \mathrm{~min}$. Simultaneously, blood samples were taken for arterial and central venous blood gases. Two temperature probes were used to measure peripheral (toe) and central (rectal) temperature (20). As is standard practice at our institution, pulmonary artery catheters were not placed routinely for these cardiac surgical operations. In addition to the above, oesophageal Doppler ultrasonography (ODM II, Abbott, Maidenhead, UK) was performed during the first 4 hrs postoperatively or until endotracheal extubation. At $24 \mathrm{hrs}$ postoperatively, a further Doppler examination was made from the suprasternal approach (ODM I, Abbott). These measurements were all made by one experienced operator using a $4 \mathrm{MHz}$ Doppler ultrasound probe in the esophagus and a $2 \mathrm{MHz}$ probe at the suprasternal notch. Spectral analysis of the Doppler frequency shifts produced blood flow velocity waveforms. These were translated into volumetric estimates of stroke volume (SV) and cardiac output (CO) by an integral normogram utilising the patient's age, height and weight (21).

The patients were managed by nursing and medical staff using only routinely used haemodynamic monitoring variables, but blinded from the Doppler data, unless the data were felt to be clinically required. The staff indicated whenever they deemed an intervention to be necessary, what the indication and intervention were, and whether they felt the set goal of correction was achieved after the therapy was instituted. Both methods were compared regarding adequacy for monitoring interventions and predicting complications.

Fluid input and output, and the use of vasoactive drugs were recorded during the 24-hr period. Patients were monitored during their in patient stay for the occurrence of organ dysfunction (as measured by the Goris MOF score (22)) and any other morbidity and mortality, for which interventions were deemed necessary. Myocardial infarction was screened and diagnosed by performing a daily 12-leads electrocardiogram or when clinically indicated. Ventricular failure was diagnosed on standard clinical and radiological grounds, plus information obtained from placement of a pulmonary artery catheter if clinically indicated.

Patients were retrospectively divided into complicated and uncomplicated postoperative outcome groups. Results were analysed by Student $t$-test, analysis of variance and chi-square testing. The validity of the different monitoring parameters were evaluated by using a method for comparing areas under the receiver operator characteristic (ROC) curves, as previously described in detail $(23,24)$. The area under the ROC-curve represents the probability that a randomly chosen diseased patient is correctly assessed for having a greater likelihood of disease than a randomly chosen non-diseased patient. Calculation of sensitivity, specificity, and positive and negative predictive values at optimal cut-off points were also performed. Optimal cut-off proints were calculated from the ROC-curve analysis as those points with the greatest 
combined sensitivity and specificity (25). Data are presented as mean \pm SD, or as frequency tables. A vallue of $p<0.05$ was considered to be statistically significant.

\section{RESULtS}

\section{Demographic data}

Twenty patients (70\% male, mean age $65.9 \pm 9.4$ years) were included in this study. Eight of the operations were emergency coronary artery bypass grafting ( $C A B G$ ), six routine $C A B G$, four were valve replacement ( 3 mitral, 1 aortic), and two combined $C A B G$ and valve replacement operations.

\section{Scoring systems}

The APACHE II score on admission to the intensive care was $14.2 \pm 8.0$ and $17.9 \pm$ 9.7 at $24 \mathrm{hrs}$. The pre-operative Parsonnet-score was $8.2 \pm 5.9$. The mean ICU stay was $2.5 \pm 1.4$ days and the mean hospitall stay was $11.4 \pm 5.4$ days.

\section{Complications}

Nine of the twenty patients $(45 \%)$ developed major postoperative complications, namely, weaning difficulties $(n=1)$, cardiogenic shock $(n=2)$, major graft bleeding $(n=1)$, septic shock secondary to chest infection and poor pre-existent condition ( $n=2)$, myocardial infarction $(n=1)$, and chest infection $(n=2)$. In five of these patients, complications did not become apparent until at least $24 \mathrm{hrs}$ postsurgery. At $24 \mathrm{hrs}$, eight patients $(40 \%)$ fulfilled the criteria of SIRS. One emergency CABG patient died after 3 days, and one routine $C A B G$ patient died after 2 days from septic shock. The overalli group mortality was thus $10 \%$. No differences could be found between patients with and without complications with respect to age, gender, APACHE-ll scores at admission and Parsonnet-score (Table 1).

\section{Standard haemodynamic variables}

Figure 1 depicts the course of routine haemodynamic variables recorded during the first 4 postoperativehrs. No differences were found at admission between the complicated and uncomplicated patient groups with respect to MAP, CVP, PaO $\mathrm{FiO}_{2}$ ratio, and core-toe temperature differences (Table 2). Over the subsequent 4 hrs, 
Table 1 Patient demographics according to the presence of complications ${ }^{\text {at }}$

\begin{tabular}{|c|c|c|c|c|c|}
\hline & \multicolumn{2}{|c|}{ With complications } & \multicolumn{2}{|c|}{ Without complications } & \multirow[t]{2}{*}{ p-value } \\
\hline Number of patients (n) & & 9 & & 11 & \\
\hline Age (years) & 66.4 & \pm 6.2 & 65.4 & \pm 11.7 & 0.4 \\
\hline Gender ( $\%$ female) & 44.4 & & 18.2 & & 0.1 \\
\hline APACHE-II (admission) & 14.8 & \pm 9.0 & 13.7 & \pm 7.5 & 0.4 \\
\hline Parsonnel-scone & 8.8 & \pm 5.4 & 7.6 & \pm 6.4 & 0.3 \\
\hline Procedure ( $\%$ emergency) & 45.5 & & 33.3 & & 0.07 \\
\hline ICU stay (days) & 2.7 & \pm 2.1 & 2.4 & \pm 0.5 & 0.3 \\
\hline Hospital stay (days) & 11.8 & \pm 5.3 & 11.0 & \pm 5.7 & 0.4 \\
\hline
\end{tabular}

aValues are displayed as mean (tstandard deviation), or as percentages. Comparison between the uncomplicated and complicated groups was done either by Student-t test or chi-square analysis. No differences coutd be found in the patient dembgraphics between the uncomplicated and complicated group.

Table 2 Clinical parameters at admission related to the development of complications ${ }^{\mathrm{a}}$

\begin{tabular}{|c|c|c|c|c|c|}
\hline & \multicolumn{2}{|c|}{ With complications } & \multicolumn{2}{|c|}{ Without complications } & \multirow[t]{2}{*}{ p-value } \\
\hline \multicolumn{5}{|c|}{ Standard haemodynamic measurements } & \\
\hline $\operatorname{MAP}(\mathrm{mmH} H)^{\circ}$ & 77.3 & \pm 9.3 & 83.2 & \pm 10.6 & 0.1 \\
\hline HR (beats/min) & 97.8 & \pm 22.4 & 78.1 & \pm 14.8 & 0.02 \\
\hline CVP (mmHg) & 9.4 & \pm 3.5 & 9.8 & \pm 3.6 & 0.4 \\
\hline $\mathrm{PaO}_{2} / \mathrm{FiO}_{2}$ & 37.6 & \pm 20.1 & 31.6 & \pm 14.7 & 0.3 \\
\hline$\Delta T\left({ }^{\oplus} \mathrm{C}\right)$ & 6.1 & \pm 3.7 & 4.6 & \pm 3.7 & 0.2 \\
\hline $\mathrm{SBE}$ & -2.2 & \pm 2.6 & 0.6 & \pm 2.1 & 0.01 \\
\hline \multicolumn{6}{|c|}{ Doppler utrasonographic measurements } \\
\hline $\mathrm{CO} \| \mathrm{l} / \mathrm{min}$ & 5.7 & \pm 2.3 & 6.0 & \pm 11.5 & 0.4 \\
\hline$S V(m)$ & 58.1 & \pm 23.3 & 79.3 & \pm 22.7 & 0.03 \\
\hline $\mathrm{FTc}$ (msec) & 0.359 & $\pm 0,05$ & 0.370 & \pm 0.05 & 0.3 \\
\hline PV (con/sec) & 56.3 & \pm 19.1 & 62.9 & \pm 15.2 & 0.2 \\
\hline
\end{tabular}

atalues are displayed as mean ( \pm standard deviation). Comparison between the uncomplicated and complicated group was done by Student-t test. The complicated group had a significant higher heart rate and lower standard base excess than the uncomplicated group. The stroke volume, measured by esophageal Doppler ultrasonography, was significantly higher in the uncomplicated group.

'MAP, mean arterial pressure; $\mathrm{HR}$, heart rate; $\mathrm{CVP}$, central venous pressure; $\mathrm{PaO}_{2} / \mathrm{FiO}$, quotient of arterial oxygen pressure and inspired oxygen pressure; $\triangle T$, core-toe temperature difference; $\mathrm{SBE}_{\text {; }}$ standard base excess; $\mathrm{CO}$, cardiac output; $\mathrm{SV}$, stroke volume; FTc, corrected llow time; PV, peak velocity. 

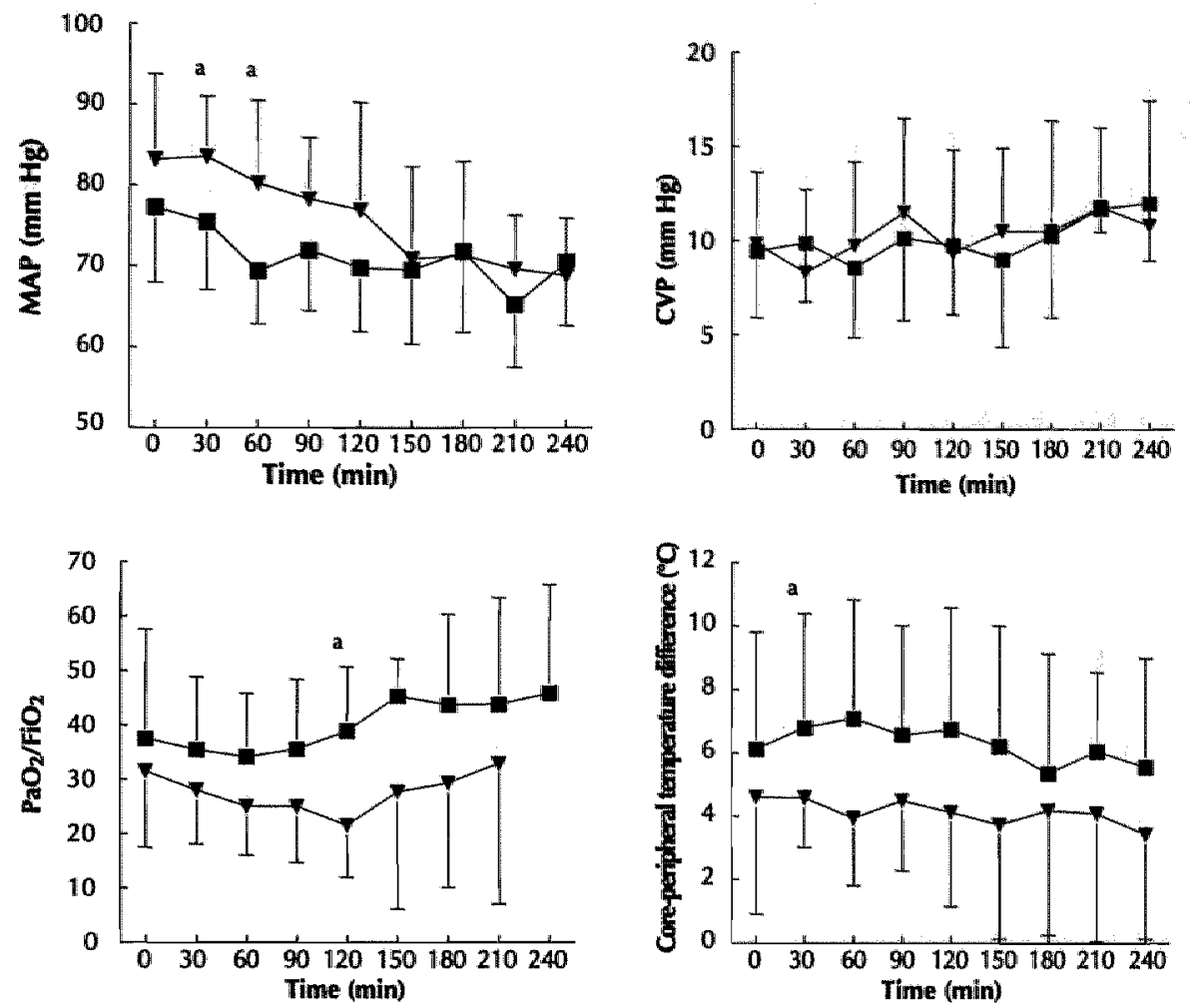

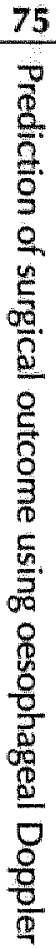
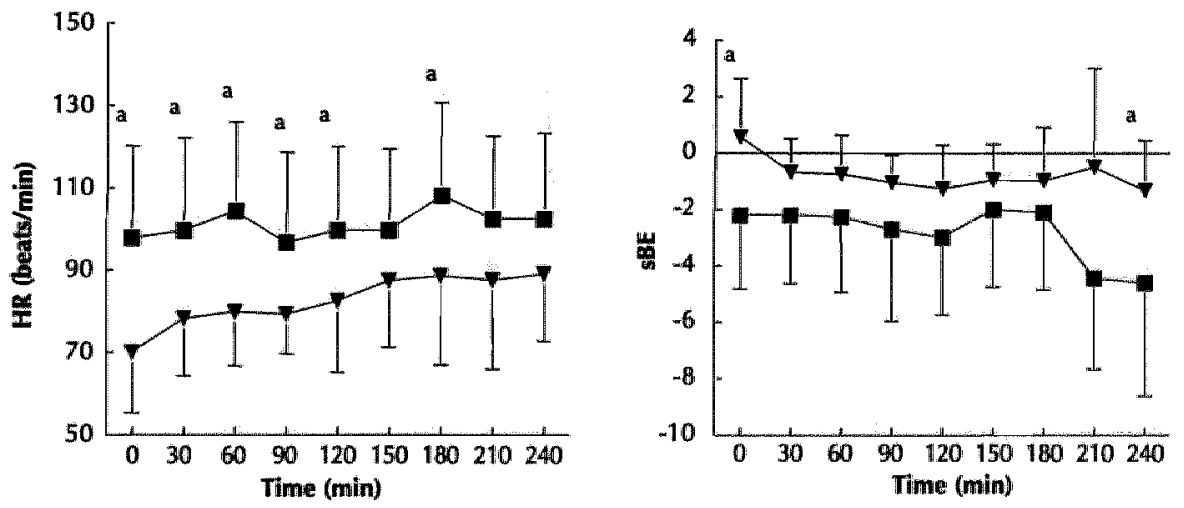

Postoperative course in standand troutinel haemodynamic variables in cardiac surgical patients. Mean arterial pressure (MAP) central venous pressure (CVP), the ratio between arterial oxygen pressure and inspired oxygen pressure $\left(\mathrm{PaO}_{2} / \mathrm{FiO} \mathrm{Z}_{2}\right)_{1}$ and the core-peripheral temperature difference $(\Delta T)$ at admission were not significantly different between the uncomplicated group (- - - and the complicated subgroup (- - The heart rate (HR) and standard base excess. (sBE) were significantly different in the two groups at admission; a $p<0.01$. 
significant differences in MAP and heart rate were found between the patient groups with and without subsequent complications $(p<0.05)$. A significantly higher coretoe temperature difference was found in the complicated group at $1 \mathrm{~h}$ post-ICU admission $(p<0.05)$. Significant differences were also found for arterial standard base deficit (sBE) between the complicated and uncomplicated groups $(p<0.05)$.
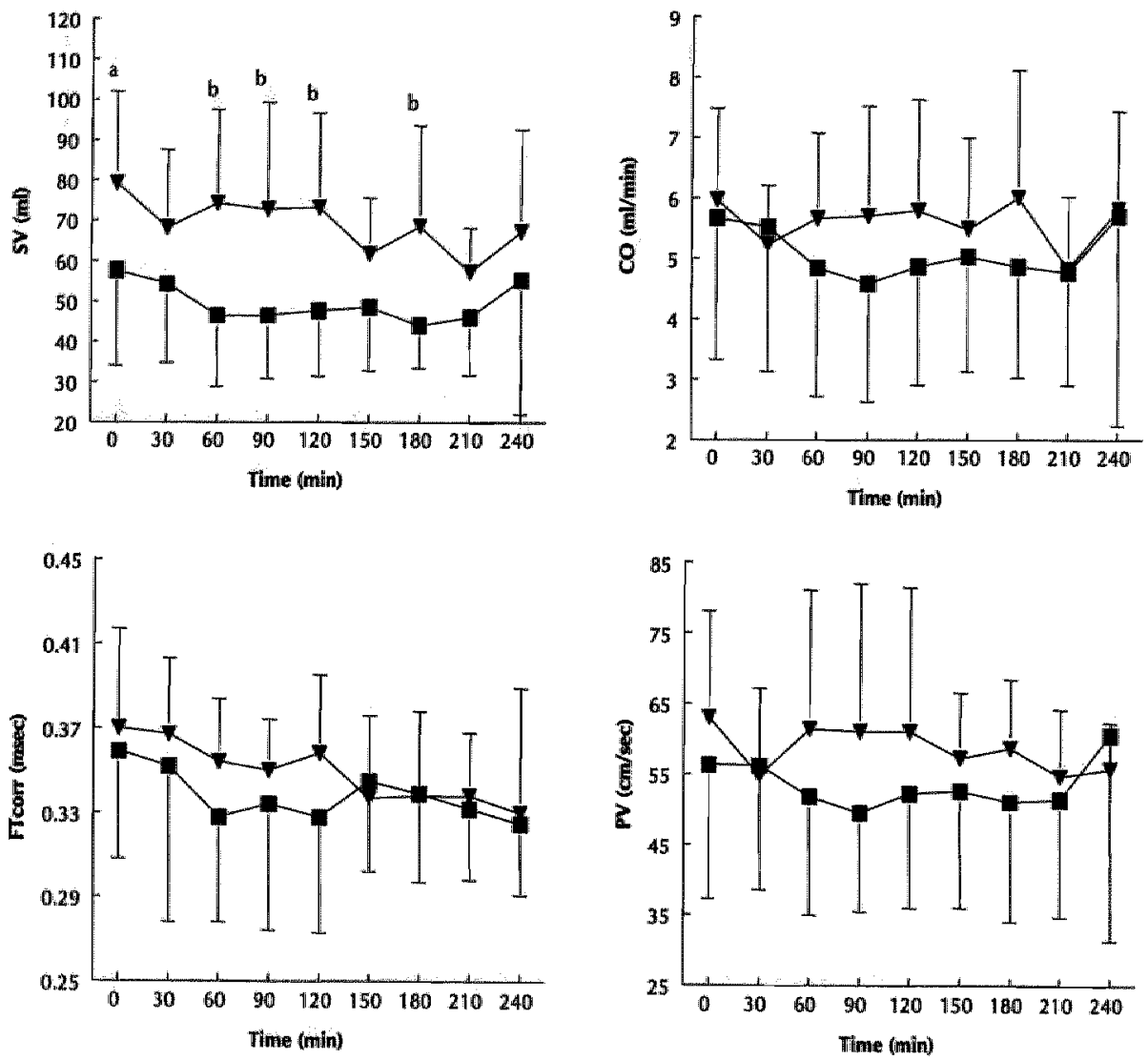

\footnotetext{
Postoperative course in oesophageal Doppler ultrasonographic parameters in cardiac surgical patients. SV (stroke volume) was significiantly higher in the uncomplicated group of patients. CO (cardiac outputt, FTc (heart rate corrected flow time), and PV lpeak velocity) did not show differences between the two subgroups; $(-\nabla-)$, uncomplicated subgroup; $(-)$, complicated subgroup; ${ }^{\mathrm{a}} \mathrm{p}<0.05, \mathrm{~b}<0.01$.
}

Figure 2 


\begin{tabular}{lllll}
\hline Area & SE & $2.5 \%$ & $97.5 \%$ \\
\hline
\end{tabular}

Standard haemodynamic measurements

$\begin{array}{lllll}\text { MAP } & 0.67 & 0.12 & 0.43 & 0.91^{\mathrm{a}} \\ \mathrm{HR} & 0.74 & 0.12 & 0.52 & 0.97^{\mathrm{a}} \\ \mathrm{CVP} & 0.55 & 0.13 & 0.28 & 0.81^{\mathrm{a}} \\ \mathrm{PaO}_{2} / \mathrm{FiO}_{2} & 0.18 & 0.12 & -0.05 & 0.41^{\mathrm{a}} \\ \Delta \mathrm{T} & 0.76 & 0.13 & 0.51 & 1.00^{\mathrm{a}} \\ \mathrm{SBE} & 0.81 & 0.10 & 0.61 & 1.01\end{array}$

Doppler ultrasonographic measurements

\begin{tabular}{lllll} 
CO & 0.61 & 0.13 & 0.36 & $0.85^{\mathrm{a}}$ \\
SV & $\mathbf{0 . 9 3}$ & $\mathbf{0 . 0 7}$ & 0.79 & 1.07 \\
FTC & 0.60 & 0.14 & 0.33 & $0.87^{\mathrm{a}}$ \\
PV & 0.76 & 0.12 & 0.52 & $0.99^{\mathrm{a}}$ \\
\hline
\end{tabular}

Data of variables is from admission data only. The maximal value for the area under the ROC-curve is 1. This represents the best identification of the randamly chosen sick patient hawing the disease compared to a randomly chosen non-diseased patient. The area under the ROC-curve from the stroke wolume was significantly langet than the area under the curves from the standard haemodynamic measurements and the other Doppler derived variables. For an expllanation of the abbreviations see table $2 ; \mathrm{a} p<0,05$.

\section{Doppler ultrasonographic measurements}

Stroke volume (SV), as measured by oesophageal Doppler, was highly predictive for the development of subsequent complications (figure 2). Both on admission and during the subsequent $4 \mathrm{~h}$ postoperative period, SV was significantly lower in patients developing complications $(p<0.05)$. Likewise, SV was significantly lower in the eight patients who fulfilled the criteria for SIRS at $24 \mathrm{hrs}$ postoperatively compared to the 12 patients without SIRS $(p<0.05)$. Because of the significant increase in heart rate in the complicated group, no difference was noted in postoperative cardiac output between the complicated and uncomplicated groups. Table 3 shows the areas under the receiver operator characteristic (ROC) curves for both routine and Doppler-measured haemodynamic variables on admission. SV had a significant larger area under the curve than either the routinely measured haemodynamic variables (except sBE) or other Doppler ultrasonographicmeasured variables, such as peak velocity or corrected flow time. By using a cutoff point of a SV of $<60 \mathrm{ml}$ at ICU admission, the sensitivity for the development of subsequent complications was $89 \%$ and the specificity $82 \%$ (Table 4). The positive 
Table 4 Quantative values of clinicall parameters at admission in predicting morbidity att the optimal cut-off point

\begin{tabular}{llll}
\hline Se $(\%)^{2}$ & Sp $(\%)$ & PPV $(\%$ & NPV $(\%)$ \\
\hline
\end{tabular}

Standard haemodynamic measurements

MAP $(<80 \mathrm{mmHg})$

$H R$ ( $>90$ bpm)

$\Delta T\left(>6.5^{\circ} \mathrm{C}\right)$

SBE $(<-0.5)$

Doppler ultrasonographic measurements

$\mathrm{CO}(<5.0 \mathrm{~V} / \mathrm{min})$

$5 V(<50 \mathrm{ml})$

$\mathrm{FTc}(\mathrm{60.355} \mathrm{msec})$

PV $(<55 \mathrm{~cm} / \mathrm{sec})$

\section{6}

67

50

67

50

89

50

63
64

82

70

73
56

75.75

$50 \quad 70$

$67 \quad 73$
64

75

The combination of the sensitivity, specificity, megative, and positive predictive value of the stroke volume measured at admission is significantly higher than the other variables. Optimal cut-aff points are shown (in parentheses)" $p<0.05$. "Se $=$ sensitivity, $S p=$ specilicity, $\mathrm{PPV}=$ positive-predictive value, $\mathrm{NPV}=$ negative predictive value.

predictive value was $80 \%$ and the negative predictive value was $90 \%$. ICU stay was significantly longer in the patients with a SV at admission $<60 \mathrm{ml}$ compared to those $>60 \mathrm{ml}(3.6 \pm 2.5$ vs. $2.2 \pm 0.6$ days; $p<0.05)$. One patient had a false-negative test result. He had a late $(>4 \mathrm{~h})$, but significant bleed from one of the grafts with profound hypotension and tachycardia. The SV decreased, but not by more than $10 \%$. Following re-operation, this patient did well with no subsequent complications.

The predictive power of measuring a SV $<60 \mathrm{ml}$ at $4 \mathrm{~h}$ and at $24 \mathrm{hrs}$ after admission was less with respect to sensitivity (respectively 62.5 and $50 \%$ ) compared to admission. However, the specificity of this test increased (80\% at 4 hrs and $100 \%$ at 24 hrs postoperatively), respectively.

\section{Interventions}

In four patients, no intervention was needed either for hypotension or for an increasing metabolic acidosis in the first $4 \mathrm{hrs}$. In 16 patients, nine interventions (fluid supplementation, inotropes) were made for hypotension (MAP range; 50 to 63 $\mathrm{mmHg}$ ) and nine (fluid suppletion, vasodilators) for an increasing arterial base deficit (sBE range; -1.9 to -7.1 ) within the first $4 \mathrm{hrs}$. Nine of these 16 patients did not achieve correction of the desired goal within the first $4 \mathrm{hrs}$. Six of these nine non-achievers still 


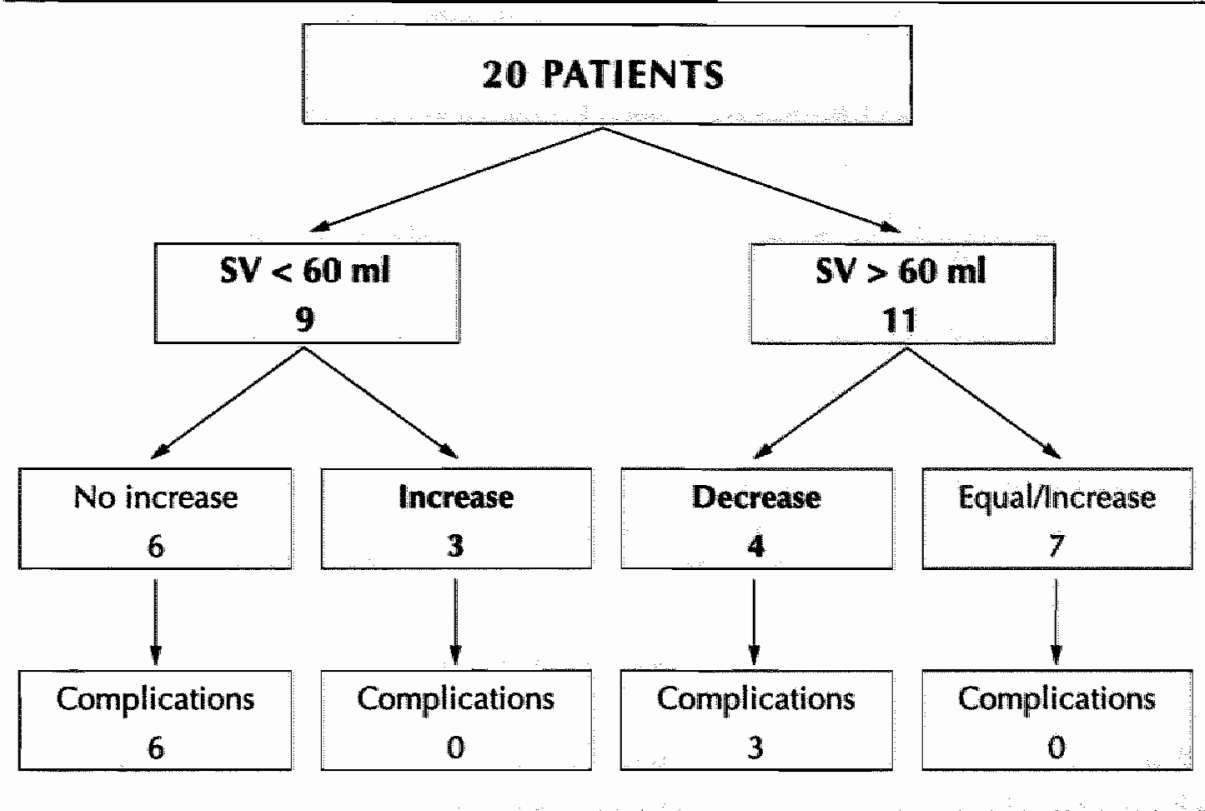

The number of post-operative complications are dependent on the admission stroke volume (SV) and the response of the SV to intensiwe care interventions.

Figure 3

had SIRS at $24 \mathrm{hrs}$ ( $\mathrm{p}<0.03$ compared with goal-achievers). The $24 \mathrm{~h} \mathrm{MOF}$ score was also higher in the non-achievers $(8.0 \pm 3.6$ vs. $4.5 \pm 2.8 ; p<0.05)$. In all patients, in whom the targets were achieved, there was an increase in SV. Of the nine patients with a $\mathrm{SV}<60 \mathrm{ml}$ at admission, six showed no improvement following interventions by $4 \mathrm{~h}$ after admission (Figure 3). All six of these patients developed complications. Of the 11 patients who had a stroke volume higher than $60 \mathrm{ml}$ on admission, four experienced deterioration during the first four hours postoperatively, despite active intervention. Three of these four patients developed complications.

At 24 hrs, no patient was hypotensive but an arterial base deficit (mean $4.8 \pm 2.3$ ) persisted in 5 patients. However, at this time point both SV and cardiac output, as measured by a suprasternally placed Doppler ultrasonographic probe, remained significantly lower $(p<0.01$ ) in patients developing complications (Table 5).

There was a significant difference in the use of vasodilators; the mean dose of nitroglycerine per patients with complications was $5.4 \pm 7.3 \mathrm{mg} / \mathrm{hr}$ compared to $9.3 \pm$ $7.0 \mathrm{mg} / \mathrm{hr}$ in the non-complicated group $(\mathrm{p}<0.001)$. Furthermore, a slight difference in the use of inotropes could be detected ( 4 patients received cardiac dose dopamine in the uncomplicated group versus 6 patients in the complicated group. The mean dose of doparmine was $1.7 \pm 1.4$ in the uncomplicated patient group compared with $2.2 \pm 1.4$ in the complicated group $(p<0.01)$. Epinephrine dosage in the complicated 


\begin{tabular}{lll}
\hline With complications & Without complications & p-value \\
\hline
\end{tabular}

Standard haemodynamic measurements

\begin{tabular}{|c|c|}
\hline 80 & CVP (mmHg) \\
\hline \multirow{3}{*}{$\frac{\frac{9}{9}}{\frac{9}{0}}$} & $\mathrm{PaO}_{2} / \mathrm{FiO}_{2}$ \\
\hline & $\Delta T\left({ }^{\circ} \mathrm{C}\right)$ \\
\hline & $\mathrm{sBE}$ \\
\hline
\end{tabular}

$\begin{array}{lcllll}\mathrm{MAP}(\mathrm{mmH} / \mathrm{g})^{b} & 77.8 & \pm 8.0 & 77.9 & \pm 5.5 & 0.5 \\ \mathrm{HR} \text { (beaths/min) } & 97.5 & \pm 18.0 & 83.3 & \pm 18.9 & 0.06 \\ \mathrm{CVP}(\mathrm{mmHg}) & 13.7 & \pm 4.6 & 14.3 & \pm 7.2 & 0.4 \\ \mathrm{PaO}_{2} / \mathrm{FiO}_{2} & 32.5 & \pm 29.1 & 53.0 & \pm 5.4 & 0.3 \\ \Delta \mathrm{T}\left({ }^{\circ} \mathrm{C}\right) & - & & 12.0 & \pm 1.3 & -\mathrm{c} \\ \mathrm{sBE} & -1.6 & \pm 2.9 & -1.8 & \pm 2.6 & 0.5\end{array}$

Doppler uttrasonographic measurements

$\begin{array}{lccccl}\text { CO }(\mathrm{l} / \mathrm{min}) & 6.3 & \pm 2.6 & 8.9 & \pm 1.35 & 0.006 \\ \mathrm{SV}(\mathrm{m}) \mathrm{l}) & 67.5 & \pm 33.1 & 111.7 & \pm 33.8 & 0.006 \\ \mathrm{FTC}(\mathrm{msec}) & 0.403 & \pm 0.12 & 0.411 & \pm 0.07 & 0.4 \\ \text { PV (cm/sec) } & 58.0 & \pm 20.9 & 86.6 & \pm 27.8 & 0.01\end{array}$

Scoringsystems

\begin{tabular}{lccccc} 
APACHE- $\|$ score & 20.9 & \pm 10.9 & 15.5 & \pm 8.6 & 0.1 \\
SIRS n (\% of patients) & 7 & $(77.8)$ & 1 & $19.1)$ & 0.001 \\
MOF-score & 8.3 & \pm 2.6 & 2.6 & \pm 2.1 & $<0.0001$ \\
\hline
\end{tabular}

avalues are displayed as mean (tstandard deviation), or as percentages. Comparison between the uncomplicated and complicated groups was done either by Student-t test or chi-square analysis.

The Doppler ultrasonographically measured cardiac oulput, stroke volume and peak velocity were significanty worse after 24 hours postoperatively in the complicated group. The complicated group had a significant higher percentage of patients lulfilling the SIRS criteria and a higher organ failure score.

${ }^{b} \mathrm{MAP}$, mean arterial pressure; $\mathrm{HR}$, heart rate; $\mathrm{CVP}$, central venous pressure; $\mathrm{PaO}_{2} / \mathrm{FiO}_{2}$, quotient of arterial oxygen pressure

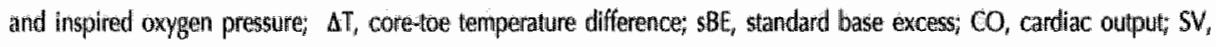
stroke vol ume; FTc, corrected flow time; PV, peak velocity; SIRS, systemic inflammatory response syndrome; MOF, multiple organ failure

Grisufficient patient numbers.

group was $5.8 \pm 3.2 \mu \mathrm{g} / \mathrm{kg} / \mathrm{min}$ compared with $0.0 \mu \mathrm{g} / \mathrm{kg} / \mathrm{min}$ in the uncomplicated group $(p<0.0001)$. At $1 \mathrm{~h}$ after admission, fluid balance was more negative $(-397 \mathrm{ml}$ \pm 133 vs. $-57 \pm 190 \mathrm{ml}$ ) in the group developing complications $(\mathrm{p}=0.042)$. This was due to a lower fluid input in the first group. At $4 \mathrm{~h}$ fluid ballance was $321 \pm 660$ versus $-79 \pm 211 \mathrm{ml}(\mathrm{p}=0.3)$, and at $24 \mathrm{~h}$ fluid balance was $636 \pm 1841$ versus $1968 \pm 167$ $(p=0.07)$ for the uncomplicated and complicated patients, respectively. 


\section{Discussion}

To our knowledge this is the first study that has prospectively monitored postoperative cardiac patients using oesophageal Doppler ultrasonography to predict subsequent complications. Despite apparent haemodynamic stability, the development of subsequent complications in these cardiac surgical patients could be predicted with $89 \%$ sensitivity and $82 \%$ specificity by a low SV $(<60 \mathrm{ml})$ on ICU admission. Cardiac output was not found to be useful as a prognostic marker in these patients. APACHE-H and Parsonnet scores were also poorly predictive, because of the high variance of the scores. Patients in whom further deterioration occurred over the subsequent 4 hrs, which failed to be corrected despite interventions, had, not surprisingly, a greater likelihood of developing problems.

Numerous pre- and intraoperative factors could be responsible for post-operative deterioration. However, for the purpose of this pilot study, that was undertaken to provide enable power calculation for a much larger study, we chose to concentrate solely on the prognostic impact of a low SV and a low cardiac output relative to other routinely measured circulatory markers. We could have selected a specific subset of patients undergoing cardiac surgery, but our aim is to find a tool which offers more widespread applicability. We are impressed at how good a distinction could be drawn with stroke volume despite relative low patient numbers.

This present study supports the end-operation findings of a previous study of 51 cardiac surgical patients by Mythen and Webb (26) in which a low gastric mucosal $\mathrm{pHi}$, a low $\mathrm{SV}$ and a high heart rate relative to those values measured in the uncomplicated patients were highly predictive for the development of post-operative complications. In other words, the subsequent development of morbidity and mortality in this patient population appears, to a large extent, to be predetermined before their ICU admission, even though standard haemodynamic variables such as arterial and central venous pressures, as well as cardiac output fall within acceptable ranges. Non-recognition of hemodynamic deterioration or failure of therapeutic interventions, especially during the first few hours, are likely to be strongly additive factors.

Management of the surgical patient demands early detection of haemodynamic deterioration and prompt correction to prevent complications and organ failure. The studies by Shoemaker et al. (27) and Boyd et al. (28) studies in high-risk surgical patients showed that perioperative hemodynamic optimisation is beneficial if commenced promptly. Their results are in marked contradistinction to the failure of 'rescue therapy' as reported by Hayes et al. (29) and Gattinoni et al. (30) in critically ill patients, of whom a large proportion were included post-operatively. Mythen and Webb, in an interventional trial performed in high-risk, non-septic, cardiac surgical patients as a follow-up to the above-mentioned observational study (26), showed that intraoperative fluid optimisation guided by the esophageal Doppler technique significantly dlecreased morbidity and hospital stay (31). Similar findings were reported by Sinclair et al. (32) using Doppler-guided fluid loading in patients undergoing surgery for fractured neck of the femur. These studies suggest that high-intensity patient care must begin at, or preferably prior to, surgery in 
order to have significant impact upon morbidity and mortality. Otherwise, good intensive care would appear simply to prevent deterioration in good-prognosis patients rather than impact upon the poor-prognosis population.

Even with the relatively small numbers studied, we found a statistically significant difference in SV, but not in cardiac output, for the development of complications. However, contemporary teaching emphasises the recognition and subsequent manipulation of a low cardiac output rather than a low SV. Tachycardia, unless striking, is also seldom commented upon and few authorities recommend fluid loading to an optimal SV rather than an 'optimal' wedge or CVP.

The significant higher incidence of SIRS after $24 \mathrm{hrs}$ and the higher rate of organ failure was found in patients with lower SVs. By contrast, measurement of cardiac output could not indicate which patient would subsequently develop complications. In all patients in whom the set goals were achieved, the $S \mathrm{~V}$ returned to normal values, and a lower frequency of SIRS and organ failure could be observed. Measurement of SV by esophageal Doppler ultrasonography appears to be useful in monitoring post-operative care.

It could be argued that the complication rate seen in our patients is exceptionally high and that, possibly, they were disadvantaged by the non use of a pulmonary artery catheter perioperatively. To give some perspective, our institution has a mortality rate of less than $1 \%$ for uncomplicated elective $\mathrm{CABG}$. Secondly, contemporaneous data from Duke University Medical Center (33), retrospectively analysing 2601 cardiac surgical patients over a three year period (1993-5), found a low overall mortality of $3.5 \%$, yet a complication rate of $37 \%$. One fourth of their patients required hospitalisation for more than 8 days, most manifesting organ dysfunction. Their complication rate, despite commonplace pulmonary artery catheter usage, is strikingly similar to ours and suggests that the low mortality routinely associated with cardiac surgery is not necessarily reflective of morbidity. Thus, in this study, we have shown that oesophageal Doppler ultrasonography appears to be a useful tool for detecting occult hemodynamic deterioration and predicting subsequent problems. Routinely monitored haemodynamic variables were not as sensitive or specific. If these results can be confirmed by a much larger study, it would be interesting to see whether oesöphageal Doppler ultrasonography can be used to guide therapy and improve outcome, either with or without a pulmonary artery catheter (34). 


\section{REFERENCES}

1. Shoemaker $W C_{,}$Wo $C C$, Bishop $M H_{\text {, }}$ et al. Noninvasive physiologic monitoring of high risk surgical patients. Arch Surg 1993; 131: 732-737.

2. Abou-Khalil $B$, Scalea $T M$, Trooskin $S Z$, et al. Hemodynamic responses to shock in young trauma patients: need for invasive monitoring. Crit Care Med 1994; 22(4): 633-639

3. Pittet $D$, Thievent $B$, Wenzel RP, et al. Importance of pre-existing co-morbidities for prognosis of septicemia in critically ill patients. Intensive Care Med 1993; 19(5): 265-272

4. Bone RC. Toward a theory regarding the pathogenesis of the systemic inflammatory response syndrome: What we do and do not kmow about cytokine regulation. Crit Care Med 1996; 24(1): 163-172

5. Deitch EA. Multiple organ faillure: Pathophysiology and potential future therapy. Ann Surg 1992; 216: $117-134$

6. Shah KB, Rao TLK, Laughlin S, et al. A review of pulmonary artery catheterisation in 6,245 patients. Anaesthesiology 1984; $61: 271$.

7. Lehman U, Amrstrong VW, Schutz $E$, et al. Monoethylglycine-xylide as an early predictor of posttraumatic multiple organ failure. Ther Drug Monit 1995; 17:125-132.

8. Putterman CE. The Swan-Ganz catheter: a decade of hemodynamic monitoring. I Crit Care 1989: $4: 127$

9. Rosenwasse $\mathrm{RH}$, Jallo $\|_{*}$, Gretch $\mathrm{CC}_{t}$ et all, Complications of Swan-Ganz catheterization for hemodynamic monitoring in patients. with subarachnoid hemorrhage. Neurosurgery 1995; 37(5): $872-875$.

10. Bull DA, Neumayer LA, Hunter GC, et al. Improved sterille technique diminishes the incidence of positive line cultures in cardiowascular incidence. I Surg Res 1992; 52(2): 106-110.

11. Connors AF, Speroff $T$, Dawson NV, et all. The effectiveness of right heart catheterization in the initial care of critically ill patient. IAMA 1996;276:889-897

12. Wong $\mathrm{DH}$, Tremper $\mathrm{KK}$, Stemmer $\mathrm{EA}$, et all. Noninvasive cardiac output: simultaneous comparison of two different methods with thermodilution. Anesthesiology 1990; 72: 784-790.

13. Wong $\mathrm{DH}_{3}$ Onishi $\mathrm{R}_{*}$ Tremper $\mathrm{KK}_{\text {, }}$ et al. Thoracic bioimpedance and Doppler cardiac output measurement: learning curve and interobserver reproducibility. Crit Care Med 1989; 17(11): 1194-1198.

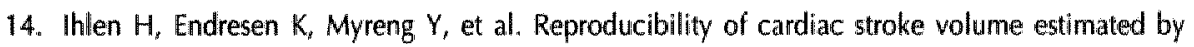
Doppler echocardiography. Am J Cardiol 1987; 59: 975-978.

15. Singer $M$, Allen $M$, Webb $A R$, et al. Effects of alterations in left ventricular filling, contractility, and systemic vascular resistance on the ascending blood velocity waveform of normal subjects. Crit Care Med 1991: 19(9): 1138-1145.

16. Mark NB, Steinbrook RA, Gugino LD, et all. Continuous noninvasive monitoring of cardiac output with esophageal Doppler ultrasound during cardiac surgery. Anesth Analg 1986; 65: 1013-1020.

17. Nashef $S A M$, Carey $F_{n}$ Silcock $M M$, et al. Risk stratification for open theart surgery: trial of the Parsonnet system in a British hospital. Brit Med J 1992; 305: 1066-1067.

18. Parsonnet $V$, Dean D, Bernstein A.D. A method of uniform stratification of risk for evaluating the results of surgery in acquired adult heart disease. Circulation 1989; 79 (suppl 1): 1-3-1-12.

19. Bone RC, Balk RA, Cerra FB, et al. ACCP/SCCM Consensus Conference. Definitions for sepsis and organ failure and guidelines for the use of innovative therapies in sepsis. Chest 1992; 101(6): 1644-1655. 
20. Woods $\mathrm{I}_{*}$ Wilkins $\mathrm{RG}_{n}$ Edwards JD, et al. Danger of using core/peripheral temperature gradient as a guide to therapy in shock. Crit Care Med 1987; 15(9); 850-852.

21. Singer $M$, Beninett $D$. Hemodynamic monitoring using aartic Doppler. In: Vincent $\mathbb{L}$ (ed). Yearbook of Intensive Care and Emergency Medicine. 1994, pp 417-429.

22. Goris J. Multiple-organ failure: Cenieralised autodestructive inflammation. Arch Surg 1985; 21 : 1190

23. Hanley $J A$, McNeil $B]$. The meaning and use of the area under a receiver operating characteristic (ROC) curve. Radiology $1982 ; 143(1): 29-36$.

24. Hanley $\mid A$, McNeil $B]$. A method of comparing the areas under receiver operating characteristic curves derived from the same cases. Radiology 1983; 148: 839-843.

25. Dwyer AJ . In pursuit of a piece of the ROC. Radiology 1997; 202:621-625.

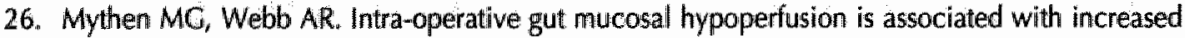
post-operative complications and cost. Intensive Care Med 1994; 20: 99-104.

27. Shoemaker WC, Appel $\mathrm{PL}_{s} \mathrm{Kram} \mathrm{HB}$, et al. Prospective trial of supranormal values of survivors as therapeutic goals in high risk patients. Chest, 1988; 94 : 1176-1186.

28. Boyd $\mathrm{O}$, Grounds RM, Bennet $\mathrm{ED}$. A randomized trial of the effect of deliberate perioperative increase of oxygen delivery on mortality in high-risk surgical patients. JAMA 1993; 270: 2699-2707.

29. Hayes $M A$, Timmins $A C$, Yau $E H S_{x}$ et al: Oxygen transport patterns in patients with sepsis syndrome or septic shock: influence of treatment and relationship to outcome. Crit Care Med 1997; 25(6): 926-936.

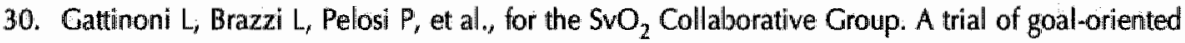
hemodynamic therapy in critically ill patients. New Eng J Med 1996; 333: 1052-32.

31. Mythen MG, Webb AR. Prospective, randomized study in patients undergoing elective cardiac surgery. Arch Surg 1995; 130: 423-429

32. Sinclait $S_{n}$ James $S_{n}$, Singer $M$. Intraoperative intravascular volume optimalisation and lenght of stay after repair of proxinal femure fracture: randomised controlled trial. Brit Med I 1997; 315: 909-912.

33. Atwell DM, Welsby I, White WD, King SA, Mythen MG. Postoperative complications following cardiac surgery with candiopulmonary bypass. Crit Care 1997; 1 (Suppl. 1): p57 (Abstract).

34. Pulmonary Artery Catheter Consensus Conference Participants. Pulmonary Artery Catheter Consensus Conference: Consensus Statement. Crit Care Med 1997; 25(6): 910-925. 


\section{ABSTRACT}

Background: Major abdominal surgery carries a high risk of serious postoperative complications and mortality. Previous studies in high-risk surgery have suggested that both mortality and serious complications can be markedly reduced by therapy aimed at maintaining increased cardiac output and oxygen delivery periand postoperatively. We have tested in a randomised, controlled, double-blind nulticenter trial the hypothesis that dopexamine, when given to fluid resuscitated patients starting before the operation and continued for $24 \mathrm{hrs}$ after surgery reduces postoperative martality and morbidity in high-risk patients undergoing major abdominal surgery.

Methods, 412 patients received either placebo $(n=140)$, dopexamine at 0.5 $\mu \mathrm{g} / \mathrm{kg} / \mathrm{min}(\mathrm{n}=135)$, or dopexamine $2.0 \mu \mathrm{g} / \mathrm{kg} / \min (n=137)$ after preoperative hemodynamic stabilisation. The primary outcome variable was mortality at 28 days. Analysis was by intention to treat.

Results. Dopexamine had no effect on mortality (at 28 days $13 \%, 7 \%$, and $15 \%$ for the group receiving placebo, dopexamine at $0.5 \mu \mathrm{g} / \mathrm{kg} / \mathrm{min}$ and dopexamine at $2.0 \mu \mathrm{g} / \mathrm{kg} / \mathrm{min}$, respectively), despite the expected, dose-dependent haemodynamic responses. No effect was observed either on the occurence of organ dysfunction, duration of intensive care, or length of hospital stay.

Discussion: We conclude that preoperative stabilisation of haemodynamics with fluids and increasing the cardiac output and oxygen delivery further by dopexamine peri- and postoperatively does not improve the outcome after major abdominal surgery as compared to fluids alone. Based on posthoc subgroup analysis and stratification according to the number of risk factors, we suggest that the concept should be further tested in patients at higher risk of complications or undergoing emergency surgery. 


\section{INTRODUCTION}

A minority of patients undergoing major surgery has a distinctly increased risk of serious postoperative complications and mortality. Since the care of these patients requires a disproportionate amount of healthcare resources, several attempts have been made to detect the patients at risk and to reduce the risk of postoperative morbidity and mortality by improved peri- and postoperative care (1-9). Despite the inherent multifactorial origin of postoperative complications, compromised physiologic reserves in combination with extensive surgery seem to be a hallmark of a high complication rate $(5,10,11)$. Major surgery increases the metabolic demands. The inability to respond by increasing cardiac output and oxygen delivery early during the peri- and postoperative course has been proposed as one of the mechanism predisposing to complications. This concept has been supported by observations that maintaining increased oxygen delivery by infusion of fluids and inotropic drugs leads to a dramatic reduction of major postoperative complications and mortality $(2,4)$. Shoemaker et al. proposed a set of simple clinical criteria to recognize the patients at risk (2). In a randomised, controlled study, the mortality of high risk patients recognized by these criteria could be reduced from $33 \%$ to $4 \%$ by increasing oxygen delivery and consumption by fluids and various vasoactive agents to the median levels of survivors of a previous descriptive series. Using a similar approach but dopexamine as the inotropic drug, Boyd et al. and Wilson et al. also demonstrated a major reduction of postoperative morbidity and mortality in high-risk surgical patients $(4,9)$. Dopexamine is a relatively new inotropic and vasodilating agent, with predominantly $\beta$-adrenergic activity. The pharmacologic properties of the drug and the promising results of Boyd et al. (4) and by Wilson et al. (9) make it an attrative alternative for perioperative haemodynamic support.

Improving the outcome of a specific high-risk surgical patient population by relatively simple means would be highly desirable from a purely clinical standpoint and also considering appropriate resource allocation. Both the actual rate of postoperative complications and the importance of different risk factors and prophylactic interventions may vary highly between different clinical settings. We, therefore, tested the concept of improving the postoperative outcome by perioperative haemodynamic stabilisation in a multinational, multicenter study. The aim of this prospective, randomised, controlled, double-blind study was to test the hypothesis that dopexamine, when given to fluid-resuscitated patients preoperatively and continued for 24 hrs after surgery, reduces postoperative mortality and morbidity in high-risk patients undergoing major abdominal surgery. 


\section{PATIENTS AND METHODS}

Thirteen hospitals from six European countries participated in the study. The protocol was approved by the appropriate Ethic Committees, and a written, informed consent was obtained from the patient, or when appropriate, from the family. A total of 433 patients were randomised. Of these, 412 patients received the study drug, and underwent surgery. These 412 patients were included in the intentionto-treat analysis. The 21 randomised patients were excluded because the surgery was cancelled and no study drug was given. No patients were excluded because of protocol violation. Patients meeting at least one of the high risk criteria as defined by Shoemaker et. al. (2) and scheduled to undergo major abdominal surgery with an expected duration of at least $1.5 \mathrm{hrs}$ were included (Table 1). The underlying diagnoses and actual operative procedures are given in Table 2 . The flow diagram of the study protocoll is illustrated in Figure 1. Briefly, after consent, patients fulfilling the entry criteria were admitted to intensive care unit (ICU) for preoperative stabilisation and study drug administration. With the aid of an arterial and a pulmonary artery catheter and blood assays, fluids, blood products and supplementary oxygen were given to obtain a cardiac output of at least $2.5 \mathrm{l} / \mathrm{min} / \mathrm{m}^{2}$, mean arterial blood pressure of $70 \mathrm{mmHg}$, pulmonary antery occlusion pressure of $10 \mathrm{mmHg}$, haemoglobin of $100 \mathrm{~g} /$, and arterial haemoglobin oxygen saturation of $94 \%$. After stabilisation, the study drug (either placebo, or dopexamine at $0.5 \mu \mathrm{g} / \mathrm{kg} / \mathrm{min}$ or $2.0 \mu \mathrm{g} / \mathrm{kg} / \mathrm{min}$ ) was started. The drugs were provided in identical vials, recognised by the patient number and in advance randomisation in blocks of six, and given to the investigators by the hospital pharmacy, who were also blinded to the actual drug given to the patient. The infusion rate of each drug was based on the body weight of the patient.

The study drug irfusion was started a minimum of $2 \mathrm{hrs}$ and a maximum of $12 \mathrm{hrs}$ before the surgery. The full infusion rate was titrated in two steps over 30 mins. In case of tachycardia (an increase in heart rate of $\geq 30 \%$ above baseline or a rate of $>130$ beats/min) during the preoperative phase, the dose was halved, and if the tachycardia persisted despite this for $>30$ mins, the study drug was stopped. During the periand postoperative phase, the clinical relevance of tachycardia and stopping the drug was determined by the clinician in charge of the patient. The overall management of haemodynamics during the operation and postoperatively was done by the clinician in charge of the patient. The drug was infused for $24 \mathrm{hrs}$ from the start of the operation. The study drug was titrated down at the end of infusion in two steps, with a 30-min interval between the two steps. The haemodynamics were followed up for another 6-12 hrs after stopping the infusion. The time points for collection of haemodynamic data were: at admission, after stabilisation, end of study drug titration, start of surgery, end of surgery, 2 hrs, 6hrs, and 24hrs after surgery and 6-12hrs after stopping the study drug infusion. If there was no clinical indication for further intensive care, the patients were then discharged to normal surgical ward, and their course followed up until hospital discharge or death. The severity of illness was assessed during the first $24 \mathrm{hrs}$ after surgery using the Acute Physiology and Chronic Health Evaluation (APACHE) II score (12). The presence of organ dysfunction was scored (13) 24hrs and 30-36hrs after surgery, and on days $3,4-6,7,14,21$ and 28 after starting the study. 


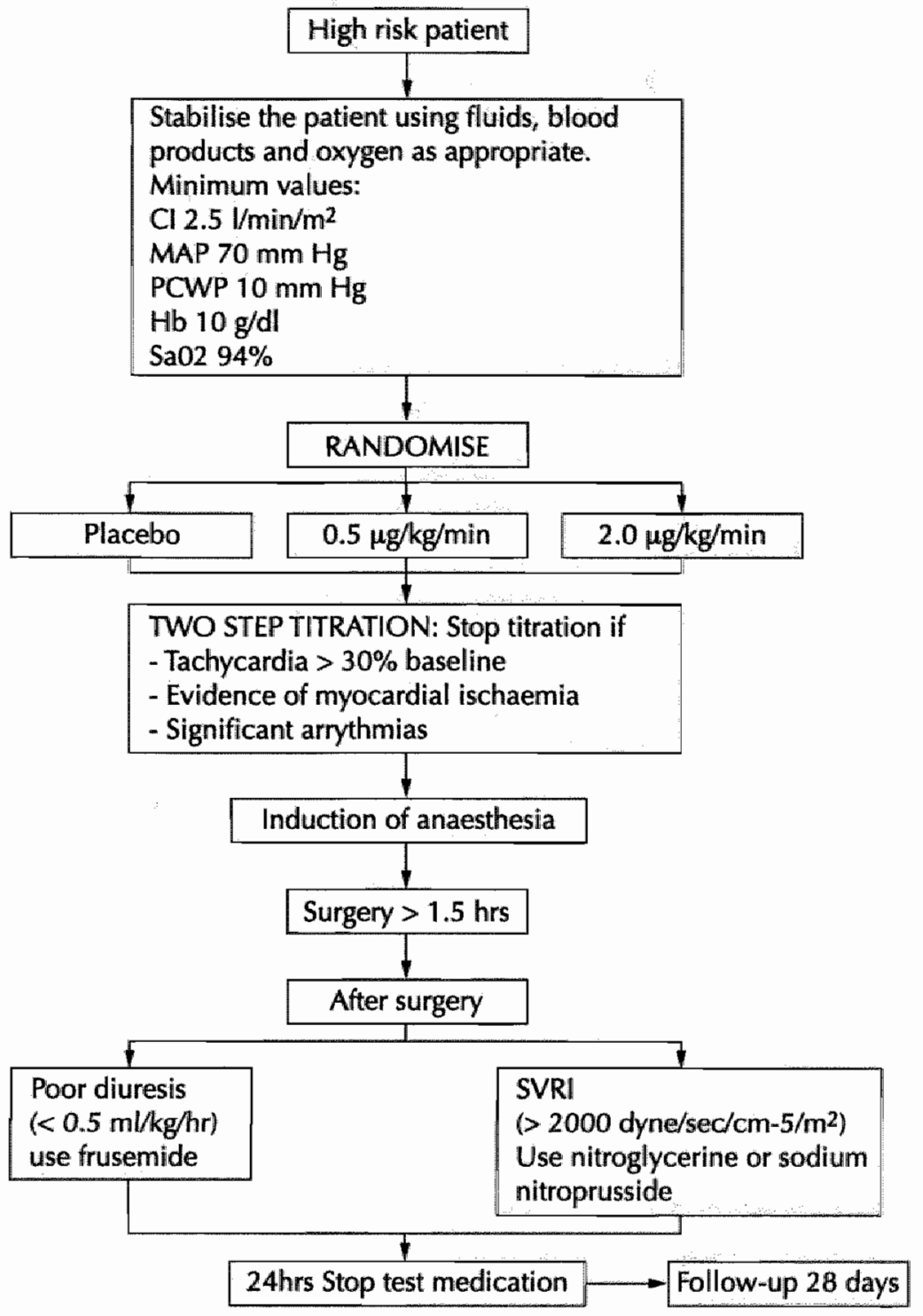


The primary outcome variable of the study was mortality at 28 days. The difference between the treatment groups was evaluated using survival analysis by the Cox's proportional hazards model. The sample size calculation assumed a 28-days mortality of $17 \%$ in the placebo group. With 135 evaluable patients in each study group (405 in total) and a significance level of $5 \%$ for between-group differences, a reduction of mortality from $17 \%$ to $6.5 \%$ would be detected with a power of $90 \%$. Nominal or binary data was analysed using the $X^{2}$-test or Fisher's exact test. Ordered categorical data were analysed by the Cochran-Mantel-Haenszel test with modified ridit scores. Where appropriate, the last observation carried forward principle was used to replace missing data. Data fulfilling the assumptions of normality were analysed using general linear models (analysis of variance). Time series analysis of haemodynamic data were performed using the calculation of the area under the curve according to the trapezoidal rule. A significance level of $5 \%$ was used. Data are given as mean (SD), unless indicated otherwise.

A post-hoc analysis for further hypothesis generation was added to the analytical plan after unblinding the data. An independent expert safety committee classified the patients into urgent and elective admissions based on blinded listings on surgical procedures and the high-risk criteria. Selected efficacy tables were analysed on these subgroups for descriptive purposes and hypothesis generation.

\section{RESULTS}

The treatment groups were well matched for the demographics, underlying diagnoses, operative procedures, and the presence of high-risk criteria (Table 1,2). The most common indication for surgery was carcinoma of the digestive organs and the most common surgical procedures included colonic and small bowel surgery, and oesophageal/gastric surgery. The majority of patients had only one high-risk criterion, and the most common risk factor was major ablative surgery for carcinoma. As expected, dopexamine resulted in dose-dependent increase in cardiac index and oxygen delivery $(p<.05)$, and decreased oxygen extraction $(p<0.01)$, whereas there was no difference between the groups in oxygen consumption (Figure 2a-d). The haemodynamic and oxygen transport differences were more prominent before and during the surgery.

The APACHE || scores during the first $24 \mathrm{hrs}$ after surgery were similar in the treatment groups: $12(6)$ in the placebo group, 11 (5) in the group receiving dopexamine at $0.5 \mu \mathrm{g} / \mathrm{kg} / \mathrm{min}$, and 12 (4) in the group receiving dopexamine at $2.0 \mu \mathrm{g} / \mathrm{kg} / \mathrm{min}$. No difference in the postoperative outcome was observed between the treatment groups (Figure 3, table 3). The mortality at day 28 (primary outcome variable) was $13 \%$ in the placebo group, $7 \%$ in the group receiving dopexamine 


\begin{tabular}{|c|c|c|c|c|}
\hline Criteria & Placebo & 0.5 & 2.0 & Overall \\
\hline Number of patients & $(n=144)$ & $(n=143)$ & $(n=146)$ & $(n=433)$ \\
\hline 1. Previous severe cardiorespiratory illness & $62(15 \%)$ & $21(15 \%)$ & $21(16 \%)$ & $20(15 \%)$ \\
\hline $\begin{array}{l}\text { 2. Extensive ablative surgery planned for } \\
\text { carcinoma or prolonged surgery of > } \\
\text { Bhrs }\end{array}$ & $111(79 \%)$ & $117(87 \%)$ & $111(81 \%)$ & $339(82 \%)$ \\
\hline 3. Severe mulliple trauma & $7(5 \%)$ & $5(4 \%)$ & $4(3 \%)$ & $55(13 \%)$ \\
\hline 4. Massive acute blood loss & $3(2 \%)$ & $0(0 \%)$ & $1(1 \%)$ & $4(1 \%)$ \\
\hline $\begin{array}{l}\text { 5. Age }>70 \text { years and evidence of limited } \\
\text { chronic physiological reserve in one or } \\
\text { more vital organs }\end{array}$ & $26(19 \%)$ & $23(17 \% !$ & $27(20 \%)$ & $76(18 \%)$ \\
\hline 6. Shock & $8(6 \%)$ & $0(0 \%)$ & $2(2 \%)$ & $10(2 \%)$ \\
\hline 7. Septicaemia/septic shock & $18(13 \%)$ & $7(5 \%)$ & $12(9 \%)$ & $37(9 \%)$ \\
\hline 8. Respiratory failure & $10 \quad(7 \%)$ & $1(1 \%)$ & $3(2 \%)$ & $14(3 \%)$ \\
\hline $\begin{array}{l}\text { 9. Acute abdominal catastrophe with } \\
\text { haemodynamic instability }\end{array}$ & $19(14 \%)$ & $9(7 \%)$ & $14(10 \%)$ & $42(10 \%)$ \\
\hline 10. Acute renal failure & $3 \quad(2 \%)$ & $0(0 \%)$ & $1(1 \%)$ & $4(1 \%)$ \\
\hline 11. Acute hepatic failure & $5(4 \%)$ & $7(5 \%)$ & $7(5 \%)$ & $19(5 \%)$ \\
\hline $\begin{array}{l}\text { 12. Late state vascular disease involving } \\
\text { aortic disease }\end{array}$ & $0 \quad(0 \%)$ & $3(2 \%)$ & $1(1 \%)$ & $4(1 \%)$ \\
\hline 13. Severe nutritional problems & $29(21 \%)$ & $24(18 \%)$ & $25(118 \%$ & $78(19 \%)$ \\
\hline Mean number of Shoemaker criteria & 1.86 & 1.61 & 1.66 & \\
\hline Number of patients with 1 high risk criterion & 66 & 82 & 81 & \\
\hline Number of patients with $\geq 2$ high risk criteria & 78. & 61 & 65 & \\
\hline Number of patients with $\geq 3$ high risk criteria & 25 & 22 & 24 & \\
\hline
\end{tabular}

at $0.5 \mu \mathrm{g} / \mathrm{kg} / \mathrm{min}$, and $15 \%$ in the group receiving dopexamine at $2.0 \mu \mathrm{g} / \mathrm{kg} / \mathrm{min}$ $(p=0.13)$. The overall mortality had the same pattern (table 3). The number of patients developing organ system dysfunction during the study was similar in the three groups (Table 4).

Serious complications were observed in 175 patients and there were no apparent differences between the treatment groups, except for higher incidence of arrythmias in the group receiving dopexamine at $2.0 \mu \mathrm{g} / \mathrm{kg} / \mathrm{min}(\mathrm{p}<0.05 ;$ Table 5). There were no differences between the groups in the duration of intensive care or hospital stay (Table 6). 
Table 2 Demographics

\begin{tabular}{lccc}
\hline Treatment Group & Placebo & $0.5 \mu g / \mathrm{kg} / \mathrm{min}$ & $2.0 \mu \mathrm{\mu g} / \mathrm{kg} / \mathrm{min}$ \\
\hline Variable, mean (SD) & $n=144$ & $\mathrm{n}=143$ & $\mathrm{n}=145$ \\
Age, years & $62.5(13.5)$ & $612(14.5)$ & $63.8(12.2)$ \\
Gender M/F & $83 / 61$ & $89 / 54$ & $87 / 59$ \\
Height, $\mathrm{cm}$ & $168(10)$ & $169(9)$ & $168(11)$ \\
Weight, $\mathrm{kg}$ & $70(15)$ & $70(13)$ & $70(14)$
\end{tabular}

Diagnosis ${ }^{\text {(n }}$ (n of patients)

Carcinoma stomach

$\begin{array}{rrr}24 & 25 & 30 \\ 22 & 18 & 19 \\ 16 & 13 & 23 \\ 14 & 20 & 7 \\ 7 & 12 & 7 \\ 7 & 7 & 6 \\ 10 & 8 & 2 \\ 9 & 3 & 5 \\ 4 & 2 & 7 \\ 1 & 6 & 2 \\ 1 & 2 & 5 \\ 1 & 2 & 5 \\ 1 & 3 & 5 \\ 46 & 34 & 30\end{array}$

Operative procedureal

Oesophagealitotal gastrectomy $\quad 35 \quad 33 \quad 48$

Hepatic 13

139

Small bowel 13

$13 \quad 12$

Large bowel

$46 \quad 45 \quad 35$

Pancreatic

20

$23 \quad 24$

Miscellaneous $5^{b}$

13

8

9

apatients may have more than one crileria

Wephrectomy/splenectomy/laparotomy/drainage/prostatectomy/resection of digestive carcinoma 

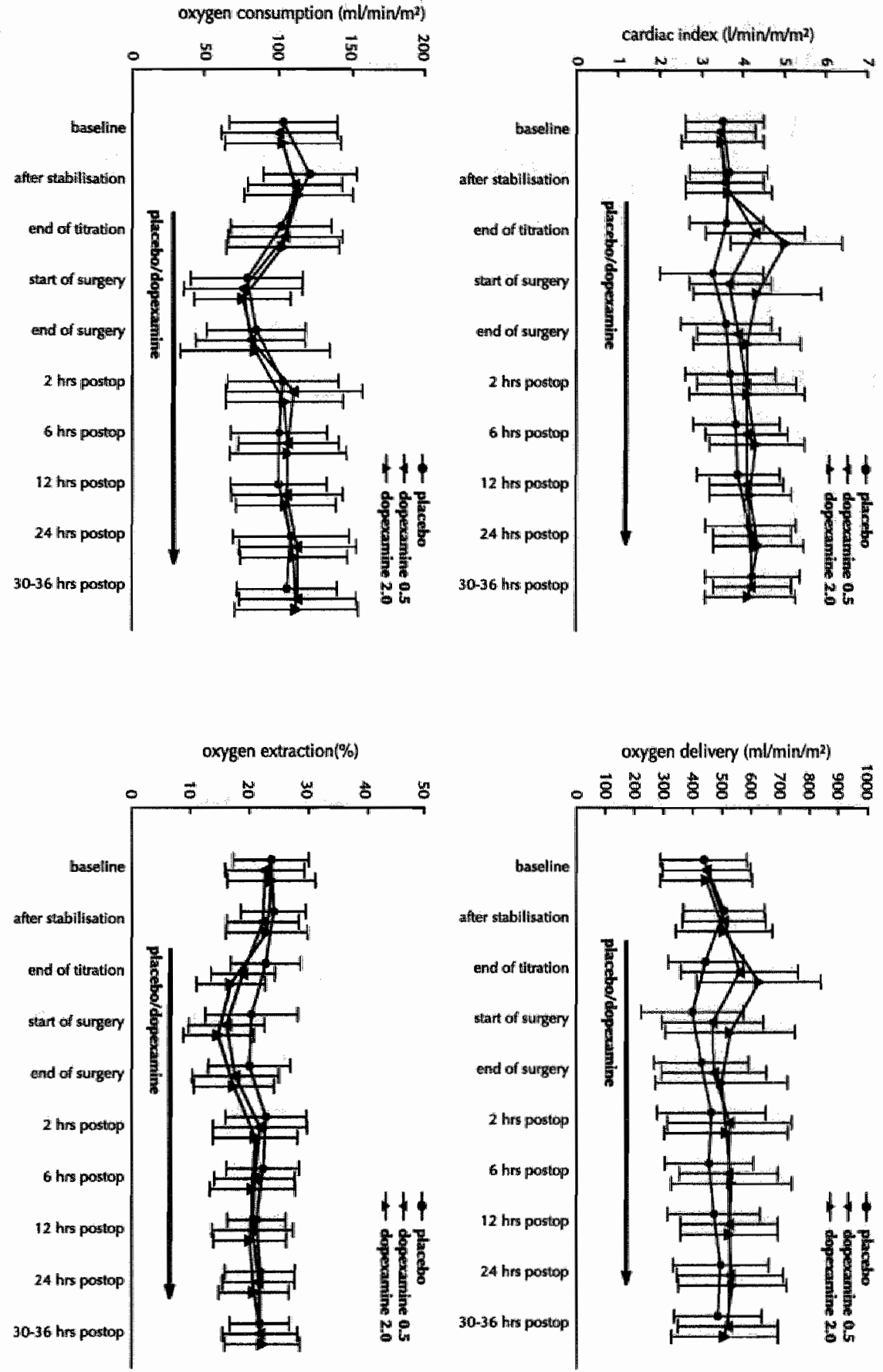


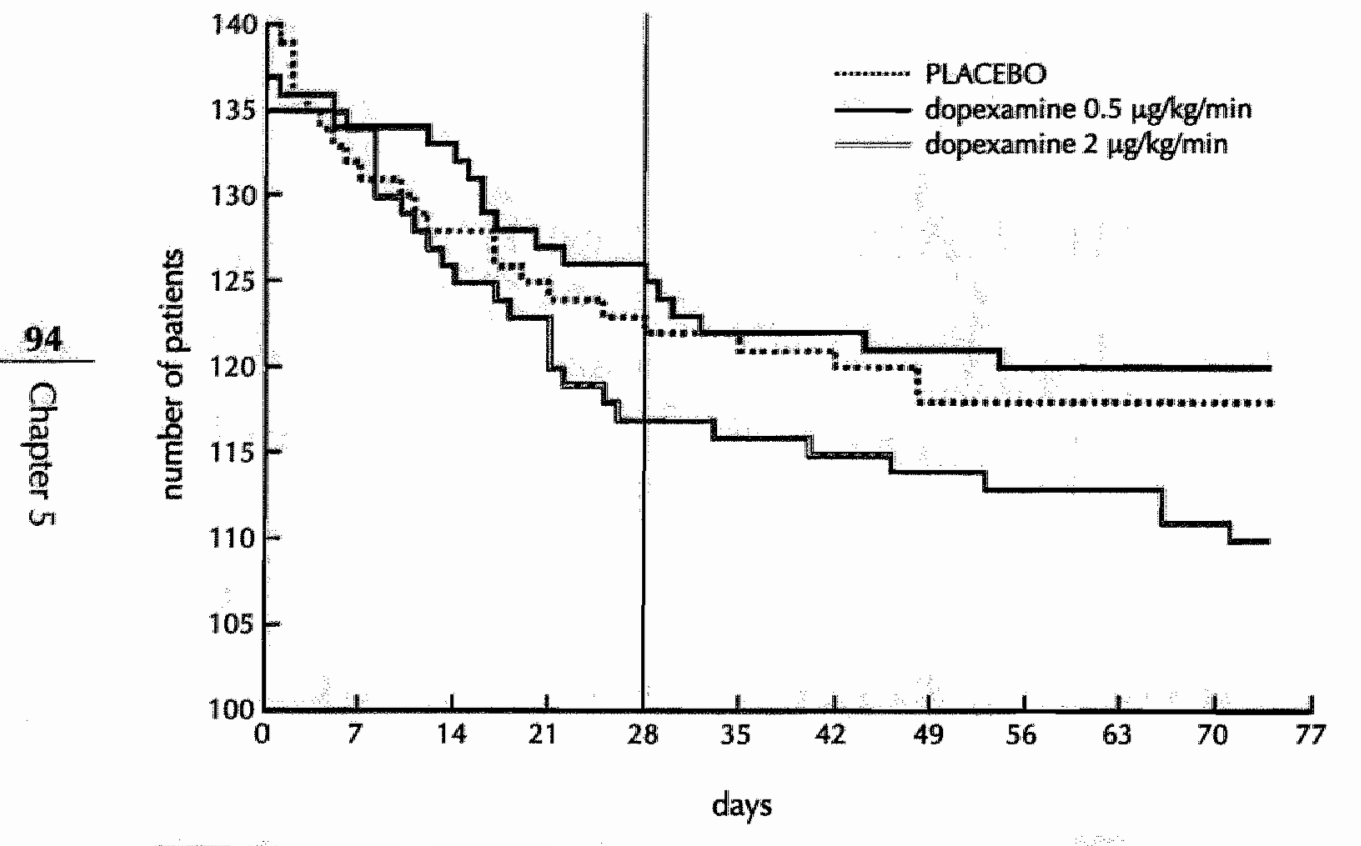

Figure 3 Survival

\section{Table 3 Mortality}

Mortality rate on day 28 and overall mortality stratified by the number of high risk criteria (2)

\begin{tabular}{|c|c|c|c|}
\hline & $\begin{array}{l}\text { Placebo } \\
(n=140)\end{array}$ & $\begin{array}{c}0,5 \mu g / k g / m i n \\
(n=135)\end{array}$ & $\begin{array}{c}2,0 \mu \mathrm{g} / \mathrm{kg} / \mathrm{min} \\
(\mathrm{n}=137)\end{array}$ \\
\hline Day 28 mortality & $18 \quad(13 \%)$ & $10 \quad(7 \%)$ & $20(15 \%)$ \\
\hline Overall mortality & $22 \quad(16 \%)$ & $15 \quad(11 \%)$ & $27 \quad(20 \%)$ \\
\hline 1 high risk criterion & $2 \quad(3 \%)$ & $4 \quad(5 \%)$ & $3 \quad(4 \%)$ \\
\hline$\geq 2$ high risk criteria & $16 \quad(21 \%)$ & $6(10 \%)$ & $17 \quad(27 \%)$ \\
\hline$\geq 3$ high risk criteria & $7 \quad 28 \%\}$ & $3 \quad(14 \%)$ & $7 \quad(29 \%)$ \\
\hline $\begin{array}{l}\text { Other risk factors than extensive } \\
\text { cancer surgery alone }\end{array}$ & $16 \quad(19 \%)$ & $6 \quad\langle 9 \%$ & $17(24 \%)$ \\
\hline
\end{tabular}


Table 4 Organ system dysfunctions

Number of patients with an ongan system dysfunction (13) at any time during the study

\begin{tabular}{|c|c|c|c|}
\hline Organ system dysfunction & $\begin{array}{l}\text { Placebo } \\
(n=140)\end{array}$ & $\begin{array}{c}0.5 \mathrm{Hg} / \mathrm{kg} / \mathrm{min} \\
(\mathrm{n}=135)\end{array}$ & $\begin{array}{c}2.0 \mu \mathrm{g} / \mathrm{kg} / \mathrm{min} \\
(\mathrm{n}=137)\end{array}$ \\
\hline Pulmonary & $43(31 \%)$ & $41 \quad(30 \%)$ & $48 \quad(35 \%)$ \\
\hline Cardiac & $30(21 \%)$ & $29(21 \%)$ & $37 \quad(27 \%)$ \\
\hline Renal & $26(19 \%)$ & $24(18 \%)$ & $29(21 \%)$ \\
\hline Hepatic & $102(73 \%)$ & $101(75 \%)$ & $103(75 \%)$ \\
\hline Haematologic & $18(13 \%)$ & $15 \quad(11 \%)$ & $18(13 \%)$ \\
\hline Gastrointestinal & $87 \quad(62 \%)$ & $67(50 \%)$ & $85 \quad(62 \%)$ \\
\hline Central nervous system & $19(14 \%)$ & $12 \quad(9 \%)$ & $13 \quad(9 \%)$ \\
\hline
\end{tabular}

Table 5 Serious adverse events.

\begin{tabular}{lccc}
\hline & $\begin{array}{c}\text { Placebo } \\
(\mathrm{n}=140)\end{array}$ & $\begin{array}{c}0.5 \mu \mathrm{g} / \mathrm{kg} / \mathrm{min} \\
(\mathrm{n}=135)\end{array}$ & $\begin{array}{c}2.0 \mu \mathrm{\mu g} / \mathrm{kg} / \mathrm{min} \\
(\mathrm{n}=137)\end{array}$ \\
\hline Anastomotic leakage or insufficiency & 14 & 6 & 8 \\
Abscess & 9 & 7 & 8 \\
Peritonitis & 5 & 4 & 3 \\
Cardiac failure & 8 & 3 & 9 \\
Circulatory failure & 6 & 6 & 7 \\
Cardiac arrest & 2 & 5 & 6 \\
Arrythmias & 9 & 12 & 21 \\
Myocardial infarct or ischaemia & 2 & 5 & 4 \\
Pneumonia & 7 & 9 & 12 \\
Sepsis & 14 & 12 & 17 \\
Hemorrhage & 10 & 10 & 14 \\
Reoperation or other surgery & 27 & 21 & 26 \\
Any serious adverse event & 60 & 52 & 63 \\
\hline
\end{tabular}

In the post-hoc subgroup analysis, 51 patients were classified as requiring urgent surgery $(21,12$ and 18 patients in the placebo, dopexamine $0.5 \mu \mathrm{g} / \mathrm{kg} / \mathrm{min}$, and dopexamine $2.0 \mu \mathrm{g} / \mathrm{kg} / \mathrm{min}$ group, respectively). The mortality at 28 days was $29 \%$ (6 patients) in the placebo group, $0 \%$ in the dopexamine $0.5 \mu \mathrm{g} / \mathrm{kg} / \mathrm{min}$ group, and $11 \%$ (2 patients) in the dopexamine $2.0 \mu \mathrm{g} / \mathrm{kg} / \mathrm{min}$ group. 


\begin{tabular}{|c|c|c|c|c|c|c|}
\hline & \multicolumn{2}{|c|}{$\begin{array}{l}\text { Placebo } \\
(n=140)\end{array}$} & \multicolumn{2}{|c|}{$\begin{array}{c}\text { Dopexamine } \\
0.5 \mu g / \mathrm{kg} / \mathrm{min} \\
(\mathrm{n}=135)\end{array}$} & \multicolumn{2}{|c|}{$\begin{array}{c}\text { Dopexamine } \\
2.0 \mu g / k g / m i n \\
(n=137)\end{array}$} \\
\hline Survivors & 6 & $(2-7)$ & 5 & $(1-5)$ & 6 & $(1-6)$ \\
\hline Non-survivors & 6 & $(1-9)$ & 10 & $(5-15)$ & 9 & $(3-15)$ \\
\hline \multicolumn{7}{|c|}{ Hospital stay days: mean (interquartile range) } \\
\hline Survivors & 24 & $(12-34)$ & 21 & $(10-22)$ & 27 & $(11-29)$ \\
\hline Non-survivors & 10 & $(2-16)$ & 15 & $(13-18)$ & 12 & $(7-19)$ \\
\hline
\end{tabular}

\section{DISCUSSION}

Risk of serious complications after surgery is perceived to be low in general. Both the clinical impression and accumulating data support the concept that there are identifiable subgroups of patients undergoing major surgery who are at high risk of developing serious postoperative complications. Mortality can approach $30 \%$, and the care of these patients consumes a disproportionate share of resources $(2,4)$. Several recent studies have suggested that improved peri- and postoperative haemodynamic management can substantially improve the outcome of high risk surgery. The successful therapeutic approaches have included fluid loading either alone or together with inotropic agents to achieve predefined haemodynamic targets $(2,4,9,14)$.

We tested the therapeutic approach of preoperative stabilisation and maintenance of increased peri- and postoperative blood flow and oxygen delivery with fluid loading and dopexamine in patients undergoing major abdominal surgery and fulfilling the previously described criteria of high risk. The main finding of this study was that dopexamine had no effect on postoperative outcome of high-risk patients undergoing major abdominal surgery compared with identical pre- and perioperative haemodynamic management of patients without dopexamine. If anything, a trend towards lower mortality in the low dose dopexamine group can be speculated.

The concept of beneficial effects of increased peri- and postoperative oxygen delivery is based on both pathophysiologic and clinical reasoning. Surgery increases the metabolic demands and an inability to respond to this by increasing cardiac output and thereby oxygen delivery predisposes the tissues to inadequate perfusion and subsequent organ dysfunction (14). The relevance of impaired physiologic reserves has been shown as an increased postoperative morbidity and mortality in patients with a low aerobic threshold in preoperative exercise testing (11). Most importantly, 
postoperative mortality has decreased from around $30 \%$ in controls to below $10 \%$ in patients, whose oxygen delivery was deliberately increased perioperatively by the use of fluids and inotropic drugs in two previous randomised, controlled singlecentre studies $(2,4)$. In contrast, increasing oxygen delivery in patients after acute admission to the ICU has not improved the outcome or has even worsened the mortality $(15,16)$. Accordingly, maintenance of increased blood flow and oxygen delivery is more likely to be beneficial when used in a preventive fashion rather than after vital organ dysfunction has already been established.

Shoemaker et al., Boyd et al. and Wilson et al. observed reductions of postoperative mortality from around $30 \%$ in controls to below $10 \%$ in patients, whose oxygen delivery was increased by the use of fluids and inotropic drugs $(2,4,9)$. The mortality of the control group in the present study was considerably lower, $13 \%$, and the treatment with dopexamine had no effect on the outcome. It is possible that the favorable results obtained in previous trials may be centerspecific and confined to centers with high mortality in the placebo/control group.

The previous studies with dramatic improvements in outcome after high-risk surgery in response to deliberate increases in oxygen delivery have included heterogenous groups of patients with a wide variety of surgical procedures and high risk factors. The potential differences in the relative importance of the different: high risk factors or type of surgery have not been addressed. Most of the patients included in this study were undergoing elective surgery and had the major ablative surgery for cancer as the most common high risk factor. The treatment groups were well matched for the presence of different risk factors and type of surgical procedures, and all underwent extensive surgery. There is no reason to assume that some underlying between-group dissimilarities would have contributed to lack of difference between the treatments.

There are some important differences between this study and the studies of Shoemaker et al. and Boyd et al. $(2,4)$, suggesting that differences in patient populations may have contributed to the different results. The mortality of the control group in this study was lower than expected based on the previous studies. As compared with the present study, the studies of Shoemaker et al. and Boyd et al. had more patients undergoing emergency surgery and the mean number of high risk criteria was higher. The relatively simple clinical criteria of high risk proposed by Shoemaker et al have not been formally evaluated to determine the relative weight of each of the criteria of increased risk. Nevertheless, they have been used with success in the previous studies of improving the outcome after high-risk surgery. Also in this study, mortality increased when the number of highrisk criteria present increased (Table 3). The mortality of patients with only one high risk criterion was very low and almost identical in the treatment groups. The trend of improved outcome in the low-dose dopexamine group can be entirely attributed to the patients with at least two high-risk criteria. Furthermore, the post hoc subgroup analysis supports the view that patients undergoing non elective surgery may be more likely to benefit from improved haemodynamic management. Another important difference between our study and the study by Boyd et al. (4) is that we used fixed doses of dopexamine in addition to fluids instead of titration to 
predefined haemodynamic end points. Nevertheless, the haemodynamic responses were very similar and, therefore, unlikely to explain the different results. In addition, the dosage of the study drug was limited because of safety considerations in $30 \%$ and $55 \%$ of the patients in the groups prescribed dopexamine $0.5 \mu \mathrm{g} / \mathrm{kg} /$ min and dopexamine $2.0 \mu \mathrm{g} / \mathrm{kg} / \mathrm{min}$, respectively. Accordinlgy, it is unlikely that using higher doses of the study drug to reach higher levels of oxygen delivery would have changed the results.

The main limitation of our study is the high number of patients with only one criterion of high risk (most undergoing elective surgery) and the associated low mortality, which may have masked potentiall benefits of adding dopexamine to preoperative stabilisation with fluids. This low mortality could not be predicted from the previous trials. It is, therefore, possible that the nonsignificant trend toward improved outcome in the low-dose dopexamine group represents the insufficient power of the study. It is also possible that some subgroup of patients may benefit from further circulatory support. So far no simple criteria for selecting such patients are available. Our results suggest that the relative weight of different combinations of high-risk criteria should be defined to improve the selection of patients for future trials.

In conclusion, we have shown that preoperative stabilisation of haemodynamics with fluids and increasing the blood flow and oxygen delivery further by dopexamine peri- and postoperatively does not improve the outcome after major abdominal surgery compared with fluids alone in patients fulfilling at least one of relatively broad high-risk criteria. Based on post-hoc analysis of the data, we propose that this therapeutic concept should be further tested in patients at higher risk of postoperative complications, such as patients with more than one high-risk criterion or high-risk patients undergoing nonelective surgery.

\section{Grant support}

This study was financed by Speywood Pharmaceuticals Ltd., England. 


\section{REFERENCES}

1. Berlauk JF, Abrams JH, Gilmour IJ, $O^{\prime}$ Connor $S R$, Knighton DR, Cerra FB. Preoperative. optimization of cardiovascular hemodynamics improves outcome in peripheral vascular surgery. Ann Surg 1991;214:289-297.

2. Shoemaker WC, Appel PL, Kram HB, Waxman K, Lee T-S. Prospective trial of supranormal values of survivors as therapeutic goals in high-risk surgical patients. Chest 1988:94:1176-86.

3. Copeland CP, Jones D, Walters M. POSSUM: a scoring systems for surgical audit. B J Surg 1991;78:355-360.

4. Boyd $O$, Grounds RM, Bennett ED. A randomized clinical trial of the effect of deliberate perioperative increase of oxygen delivery on mortality in high-risk surgical patients. JAMA 1993;270:2699-2707.

5. Shoemaker WC, Appel PL, Kram HB. Hemodynamic and oxygen transport responses in survivors and non survivors of high risk surgery. Crit Care Med 1993;21:977-990.

6. Arvidsson S, Ouchterlony J, Nilsson S, Sjöstedt L, Svärdsudd K. The Gothenburg study of perioperative risk. I. Peroperative findings, postoperative complications. Acta Anaesthesiol Scand 1994;38:679-690.

7. Arvidsson $S$, Ouchterlony I, Sjöstedt $L$, Svärdsudd K. Predicting postoperative adverse events. Clinical efficiency of four general classification systems. Acta Anaesthesiol Scand 1996;40:783-791.

8. Browner WS, Li J, Mangano DT. In-hospital and long-term mortality in male veterans following moncardiac surgery. JAMA 1992;268:228-232.

9. Wilson J, Woods I, Fawcett I, et al. Reducing the risk of major elective surgery: randomised controlled trial of preoperative optimisation of oxygen delivery. BMJ 1999;318:1099-1103.

10. Holden DA, Rice TW, Stelmach K, Meeker DP. Exercise testing, 6-min walk, and stair climb in the evaluation of patients at high risk for pulmonary resection. Chest 1992;102:1774-1779.

11. Older R, Smith R, Courtney P, Hone R. Preoperative evaluation of cardiac failure and ischemia in elderly patients by cardiopulmonary exercise testing. Chest 1993;104:701-704.

12. Knaus WA, Draper EA, Wagner DP, Zimmerman JE. APACHE If severity of disease classification system. Crit Care Med 1985;194:818-829

13. Roumen RM, Schers T], de Boer HH, Goris RJ. Scoring systerns for predicting outcome in acute hemorhagic necrotizing pancreatitis. Eur \Surg 1992,158:167\%171.

14. Shoemaker $\mathrm{WC}_{r}$ Appell $\mathrm{Pl}_{\mathrm{w}_{r}}$ Kram HB. Role of oxygen debt in the development of organ failure sepsis, and death in high-risk surgical patients. Chest 1992;102:208-15.

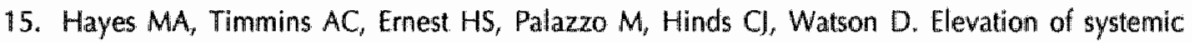
oxygen delivery in the treatment of critically ill patients. N Engl J Med 1994;330:1717-1722.

16. Gattinoni $\mathrm{L}$, Brazzi $\mathrm{L}_{\mathrm{r}}$ Pelosi $\mathrm{P}$, et al. Trial of goal-oriented hemodynamic therapy in critically ill patients. N Eng| | Med 1995;333:1025-1032. 


\section{ABSTRACT}

Background Although meta-analyses of randomised trials have shown that haemodynamic optimisation reduces montality in peri-operative patients, the influence of trail quality on the effectiveness of haemodynamic optimisation has not been rigorously evaluated.

Methods: A systematically literature review was conducted to assess the methodological quality of all individual trials using haemodynamic optimisation in peri-operative patients and in patients with sepsis and organ failure and its relation to the reported effects on mortality. An a priori division was made between the studies including peri-operative patients and studies on patients having organ failure and sepsis. Data on population, intervention, outcome and methodological quality were abstracted by two reviewers. The methodological quality was assessed using a scoring system (range 0-16) based on allocation and concealment, patient selection, end-point of intervention, extent of follow-up, comparability at baseline, cointerventions and crossovers.

Results: The mean (SD) quality of the studies was 9.1(1.8). Haemodynamic optimisation decreased mortality rate (relative risk (RR $0.45 ; 95 \% \mathrm{Cl} 0.34-0.61$ ) in peri-operative patients (17 studies). In contrast, in patients with sepsis and organ failure (10 studies) no difference in mortality rate was identified (RR $0.95 ; 95 \% \mathrm{Cl}$ $0.82-1.09$ ). When all studies combined reduction in mortality was significant (RR $0.84 ; 95 \% \mathrm{Cl} 0.77-0.92$ ). However, in all trials combined with a quality score $\geq$ 10 , no effect on mortality could be found, in contrast to studies with a score $<10$. This could not be found in high-risk surgery. In the absence of blinding no effect on mortality was present in peri-operative studies.

Conclusion: Although the quality of trials using peri-operative interventions aimed at deliberately aiming at an optimised haemodynamic profile is only moderate, a reduced mortality was found in high-risk surgical patients. Further large scale, multicentre studies are required before widespread optimisation can be recommended. 


\section{INTRODUCTION}

It has been shown that in critically ill patients an impaired cardiovascular function plays a role in the development of organ failure. Understanding which underlying mechanism was responsible for this dysfunction has changed over the past 10 years. Previously, in the treatment of surgical patients, correction of disturbed haemodynamics to normal values in the peri-operative phase was considered standard care. However, clinical signs of hypovolaemia are non-specific and nonsensitive (1). Moreover, since mean values of commonly used parameters, such as central venous pressure and pulmonary artery occlusion pressure are similar between survivors and non-survivors, correcting these parameters to normal values is questionable (2). The same was true for critically ill patients treated on the intensive care unit for sepsis (1).

The report of Shoemaker et al. changed the view on the haemodynamic treatment of the critically ill patient (3). In this report, the observation was made that 'normal' values are 'abnormal' in post-operative, trauma, and critically ill patients. Compared to non-surviving patients, surviving trauma patients had above normal oxygen delivery and oxygen consumption values. These 'supra-normal' values may represent an ability of these patients to respond adequately to the 'stress' of the trauma.

There are now a considerable number of controlled, randomised, clinical studies that have investigated the role of increasing oxygen delivery to the tissues to supranormal levels. Heyland et al. published a review in 1996 evaluating studies including patients using supranormal oxygen delivery as the goal of treatment (4). This review, including in total 1291 patients, indicated that timing of intervention may influence outcome. In high-risk surgical patients, early optimisation techniques may positively influence outcome in contrast to late intervention in patients with septic shock or multiple organ failure (4). Previously, we have emphasized the importance of separating the results from trials using early or late optimisation techniques $(5,6)$. Patho-physiologically it is known that autoregulation of the microcirculation in peri-operative and trauma patients remains intact, and the main problem is hypovolaemia or pre-existent cardiac impairment (7). In contrast, in sepsis, autoregulation is disturbed and a state of 'vasoplegia' exists. This may explain the difference in results between the two patient categories. However, this subject has remained controversial. Two recent meta-analyses by Boyd et al. and Kern et al. seem to confirm the importance of this difference between early and late intervention studies $(8,9)$.

In total a considerable number of studies have been published, totaling more than 3,000 patients. However, the quantity of studies and patients does not necessarily reflect the quality of studies. Incorporating studies, which have an inadequate approach to controlled trial design and execution, may introduce bias in the overall effect of the intervention. Unfortunately, previous meta-analyses did not use such a quality assessment in their overall outcome analysis.

Therefore, this review analysed the relationship between the methodological quality of the individual trials and the reported effects on mortality in both perioperative patients (including post-traumatic patients) and patients with sepsis and organ failure. 


\section{MATERIALS AND METHODS}

\section{Study identification}

Three methods were used to retrieve information for this review $(10,11)$. First, MEDLINE and EMBASE databases were searched for the years 1980 to 2002 , using the following mesh headings: "oxygen consumption or haemodynamics or dobutamine or fluid therapy', exploding with 'randomised controlled trials' (publication type) and 'intensive care, critical care or intensive care unit or surgery or peri-operative care'. The second method used was to search personal files and communications in order to find additional citations and to search Current Contents for recently published studies. Thirdly, the reference lists of the articles and previous meta-analyses found with the above mentioned methods were searched for additional articles and the authors of the individual trials were contacted if necessary.

\section{Study selection}

The articles, which were found using this search method were classified into original articles, reviews and others (e.g. letters). Studies were selected when representing a randomised controlled trial, using fluid and/or additional vaso-active therapy in order to optimise or maximize the haemodynamic condition of the patients (end-points: oxygen delivery, cardiac index, oxygen consumption, mixed venous oxygen saturation, and/or stroke volume). Moreover, the included studies should have been performed either in an adult intensive care unit population or an adult surgical population. Studies with zero mortality in both treatment arms were not exclucled from the meta-analysis.

Three studies were omitted from the analysis after careful review of the methodology: The study by Garrison et al. was a case-control study, in the study by Blow et al. no randomisation took place, and in the study by Fleming et al. a pseudo-randomisation was performed (37-39).

\section{Methodologic quality assessment}

A methodological scoring system (Table 1) was used to give a relative assessment of the quality of the primary selected studies (12). The scoring system was based upon the system proposed by Juni et al. (13) and used before by Heyland et al. (4). The scores for the individual studies were compared between two independent observers (M.P. and G.R.) and in case of disagreement a third (noninvolved) person decided on the score of the study. Since not all studies aimed at the reduction of mortality as primary end-point a division was made in scoring between studies aiming primarily at reducing mortality ( 2 points) and having 
a reduction in mortality as a secondary end-point (1 point). All these studies did report mortality rates. The presence of cross-over was defined as a patient achieving the haemodynamic goals of the opposite group, from the group to which the patient was allocated (e.g. patient in the control group achieving the oxygen delivery goal defined for the treatment group without additional treatment). In total, the maximum score for study quality was 16. Studies were considered highquality studies when the total score was 10 points or more. In addition several subset analyses were performed on some individual components of trial quality (see below).

Methods

\begin{tabular}{|c|c|c|c|}
\hline Randomisation & Not randomised & & Randomised \\
\hline Blinding & Not blinded & & Double blinded \\
\hline Analysis & Other & & Intention-to-treat \\
\hline End-point morlality & No end-point & Secondary end-point & Primary end-point \\
\hline \multicolumn{4}{|l|}{ Population } \\
\hline Patient selection & $\begin{array}{l}\text { Selected patients or } \\
\text { unclear }\end{array}$ & $\begin{array}{l}\text { Consecutive eligible: } \\
\text { patients }\end{array}$ & \\
\hline Comparability at baseline & No or unclear & Yes & \\
\hline Extent of follow-up & Incomplete & Complete & \\
\hline \multicolumn{4}{|l|}{ Intervention } \\
\hline Treatment protocol & Unclear & Reproducible & Well described and equal \\
\hline Cointerventions & Not described & $\begin{array}{l}\text { Described, but not equal } \\
\text { or unclear }\end{array}$ & \\
\hline Crossovers & Not described & $>10 \%$ & $<10 \%$ \\
\hline
\end{tabular}

\section{Statistical analysis}

A statistical meta-analysïs was performed using Review Manager 4.1.10. The primary outcome was the overall mortality rate reported at 28 to 30 days. The relative risk ratios for the separate studies and the overall relative risk-ratio with $95 \%$ confidence intervals (Cl) were calculated according to the method of Mantel 
and Haenszel. To assess the between-studly heterogeneity, the method according to Dersimonian and Laird was used (14). If significant heterogeneity was not found, a fixed-effects model was used to calculate pooled relative risk and $95 \% \mathrm{Cls}$. Data were primarily analysed for the results from the peri-operative and the 'sepsis' studies as proposed in the initial hypothesis and secondly analysed with all studies combined. The two patient groups (peri-operative patients and patients with sepsis and organ failure) were separated using the inclusion criteria from the original studies.

106 Several subset analyses were performed. A subset analyses were planned and conducted to investigate the effects if the following methodologic quality criteria: quality scoring, presence of mortality as end-point, blinding, and crossover.

Moreover, a subset analysis was performed including the studies using the original criteria as proposed by Shoemaker et al. (i.e. cardiac index (CI) $>4.5 \mathrm{l} / \mathrm{min} / \mathrm{m}^{2}$, oxygen delivery $\left(\mathrm{DO}_{2}\right)>600 \mathrm{ml} / \mathrm{min} / \mathrm{m}^{2}$, or oxygen consumption $\left(\mathrm{VO}_{2}\right)>170 \mathrm{ml} /$ $\left.\mathrm{min} / \mathrm{m}^{2}\right)(3,19-30,40-42)$. The other studies used a variety of therapeutic goals, among which $\mathrm{SvO}_{2}(17,29,31,32)$, left-ventricle stroke work index (30), stroke volume $(31,32)$, or lower cardiac index values than $4.5 \mathrm{l} / \mathrm{min} / \mathrm{m}^{2}(15,16,18,36)$. For this subset analysis, the study by Gattinoni et al. was divided into a two data sets (29). One included the patients, who had the $\mathrm{Cl}$ as goal of treatment. This data set was included in the studies using the original criteria as proposed by Shoemaker et al. (3). The patients who had the $\mathrm{SvO}_{2}$ as goal of treatment were included into the other study subset.

\section{RESULTS}

\section{Study inclusion and allocation}

After initial screening and subsequent more detailed evaluation of retrieved randomised trial reports, 30 canditate trials were identified. A total of 27 studies were included in the analysis, randomising a total of 3,515 patients.

Of the 27 included trials 17 involved surgical or trauma patients haemodynamically optimised peri-operatively and 10 trials involved patients with sepsis and/or organ failure. The median number of patients who underwent randomisation was 125 (range 30-762). Duration of follow-up up to 28 or 30 days could be specified in all trials.

\section{Peri-operative and trauma studies}

There were 1957 patients enrolled in the studies optimising patients peri-operatively and directly post-trauma. The overall odds ratio for mortality with haemodynamic 


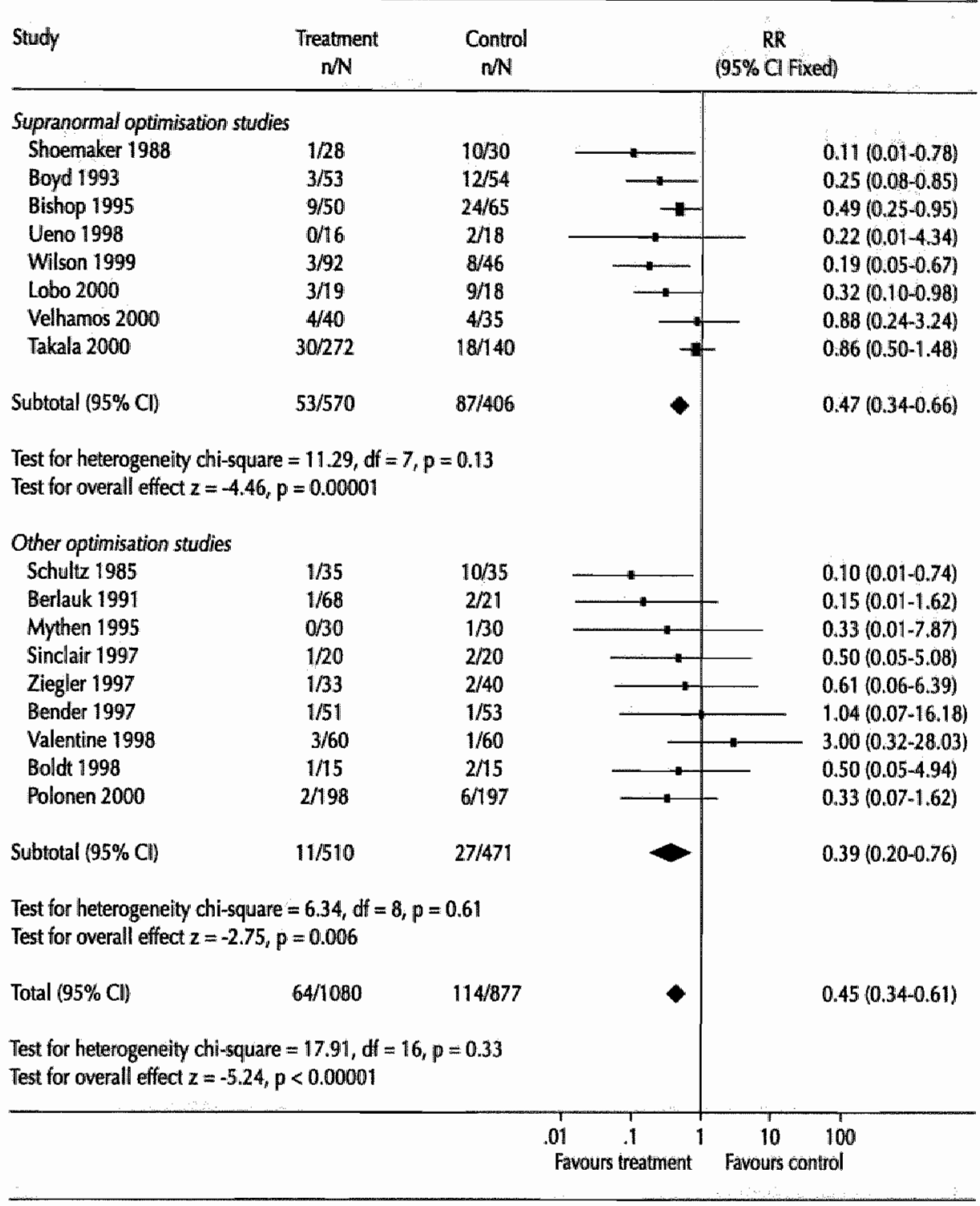

The relative risk for individual trials in studies including patients peri-operatively is shown as boxes scaled according to weigting by using the inverse variance method. Error bars indicate $95 \%$ confidence intervals. (Cl). Favours treatment denotes flavours using haemodlynamic optimisalion strategies. Fixed-effects model was used when heterogeneily analysis was not significant. The pooled relative risk estimates are shown as diamonds that span the $95 \% \mathrm{Cl}, \mathrm{n}=$ number of deceased pattients in the treatment or control arm, $\mathrm{N}=$ total number of patients in treatment or control arm, $\mathrm{RR}$ = relative risk ratio. The studies were divided into twro subsett;" studies using the Shoemaker criteria for oxygen deliwery $\left(\mathrm{DO}_{2}\right)$ and studies using other haemodynamic optimisation criteria. Total RR with the $95 \% \mathrm{Cl}$ were also depicted.

\section{Figure 1}




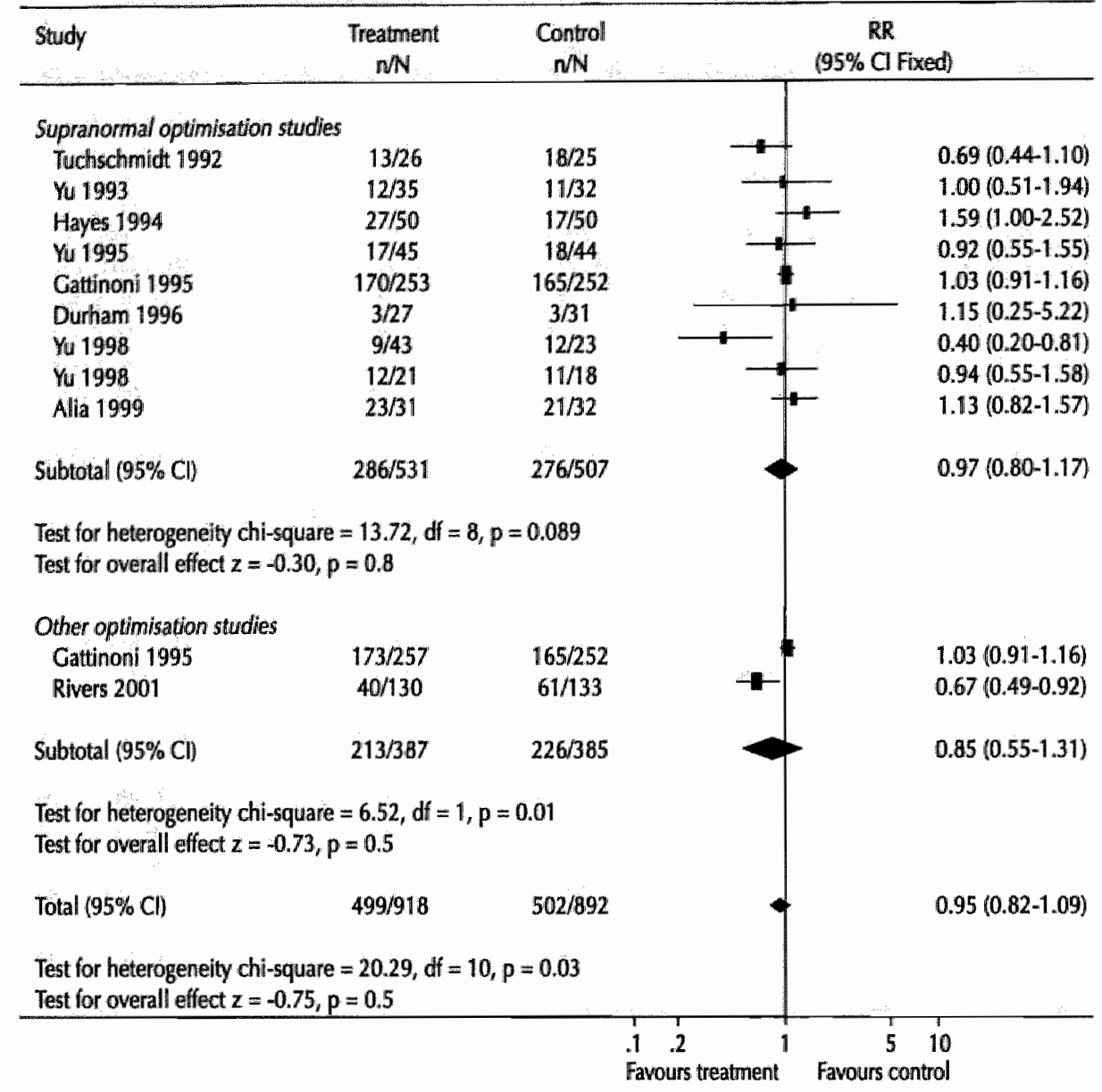

The relative risk for individual trials in studies including patients with sepsis and/or organ failure is shown as boxes scaled according to weighting by using the inverse variance method. Error bars indicate $95 \%$ confidence intervals (C). Favours treatninent denotes favours using haemodynamic optimisation strategies. A random-effects model was used when heterogeneity analysis was signilicant. The pooled relative risk estimates are shown as diamonds that span the $95 \% \mathrm{Cl}$. in = number of deceased patients in the treatment or control $a r m, N=$ total number of patients in treatment or control arm, $R R=$ relative risk ratio The studies were divided into two subsets: studies using the Shoernaker criteria for oxygen delivery $\left(\mathrm{DO}_{2}\right)$ and studies using other haemodynamic optimisation criteria. Total RR with the $95 \% \mathrm{Cl}$ were also depicted.

\section{Figure 2}

optimisation at an early stage is $0.40(95 \% \mathrm{Cl} 0.29-0.56)$ with a relative risk ratio of $0.45(95 \% \mathrm{Cl} 0.34-0.61)$ (Figure 1). These studies were homogenous on statistical grounds $\left(X^{2}=17.9\right.$, d.f. $\left.=16, p=0.3\right)$. When using an optimisation protocol 14 patients $(95 \% \mathrm{Cl} 10-23)$ have to be treated to save 1 life. 
Of the 17 studies included, 8 show a significantly greater survival in the optimised patients, the other studies did not show a significant difference in survival. The absolute risk reduction was $7.1 \%(95 \% \mathrm{Cl} 4.4-9.7 \%)$. The number of patients which should be included into a single study to be able to find this difference is 500 , assuming a mortality rate of $15 \%$ in the control group.

\section{Studies using septic/organ failure patients}

The overall odds ratio for the 1558 enrolled patients with septic shock/organ failure is $0.85(95 \% \mathrm{Cl} 0.58-1.25)$ with a relative risk ratio of $0.95(95 \% \mathrm{Cl} 0.82-1.09$; randomeffect model) (Figure 2). The random-effect model was chosen since the studies were heterogenous on clinical and statistical grounds $\left(X^{2}=20.0, d . f_{2}=9, p=0.02\right)$. Of the 10 included studies 3 studies showed either a tendency to or a significantly increased mortality in the treated patients. Two studies showed an improved survival.

\section{Summary of combined studies}

The odds ratio for all studies combined is $0.58(95 \% \mathrm{Cl} 0.42-0.80)$ with a relative risk of $0.72(95 \% \mathrm{Cl} 0.58-0.90$; random-effects model). Test of heterogeneity for all studies combined showed a significant level $\left(\chi^{2}=60.6\right.$, d.f. $\left.=26, p=0.0001\right)$. The absolute risk reduction is $1.6 \%(95 \% \mathrm{Cl} 1.5-4.6 \%)$ with a NNT of $65(95 \% \mathrm{Cl} 22-67)$.

\section{Quality assessment and subset-analysis}

The mean score for the methodology quality assessment in the included studies was 9.1 (range 5-13), 57\% of a maximum score of 16 . The mean quality score for the peri-operative studies was not different from the score of the studies in septic/ organ failure patients $(9.1$ versus $9.1 ; p=0.9$ ).

The correlation between the score and the odds ratio was not significant $(r=0.28$; $p=0.27)$ in high-risk surgical patients. The correlation between the assessment score and the odds ratio was $0.28(p=0.4)$ for studies including patients with sepsis and organ failure.

\section{Quality assessment score}

Studies (all trials combined) with a high quality assessment (score $>10$ ) had a non-significant relative risk ratio (RR $0.79(95 \% \mathrm{Cl} 0.59-1.06)$ ). The studies with a lower quality assessment score had a significant effect on mortality (RR 0.58 (95\% Cl 0.46-0.72)) (Table 2). However, no differences were found between the relative risk ratios in the studies including patients peri-operatively (RR $0.24(95 \%$ $\mathrm{Cl}=0.12-0.47)$ versus ( $\mathrm{RR} 0.51(95 \% \mathrm{Cl}=0.31-0.86$ for trial quality $<10$ points versus trial quality $\geq 10$ points). 
Table 2 Subset analyses of pooled relative risk of death

Trial categony
Relative risk $(95 \% \mathrm{Cl})$

0.47

0.39

1.00

0.85

0.77

0.69

0.79

0.58

0.24

0.51

0.74

0.36

0.47

0.36

0.64

0.75

0.61

0.32

0.50

0.82

0.25

0.55
$(0.34-0.66)$

$(0.20-0.76)$

(0.90-1.11)

(0.55-1.31)

$(0.61-0.97)$

(0.45-1.05

(0.59-1.06)

$(0.46-0.72)$

(0.12-0.47)

(0.31-0.86)

$(0.59-0.93)$

$(0.13-0.99)$

(0.35-0.65)

$(0.13-0.99)$

(0.40-1.03)

10.60-0.93

(0.36-1.04)

(0.20-0.50)

(0.29-0.86)

$(0.67-1.01)$

$(0.14-0.45)$

$(0.36-0.85)$ p-value for

heterogeneity

All trials, no crossower

Peri-operative trials, crossover

Peri-operative trials, no crossover

Fixed-effects model was used when heterogeneity analysis was not significant. $\mathrm{Cl}=\mathrm{cardiac}$ index, $\mathrm{DO}_{2}=0 \mathrm{xygen}$ delivery, $\mathrm{VO}_{2}=$ oxygen consumption

\section{Mortality end-point}

Relative risk ratios could be calculated for 25 of the $27(93 \%)$ included studies. Of the combined studies which had mortality as primary end-goal the effect on mortality tended to be lower than the effect on mortality in the remaining studies, although both subsets had a significant reduction in mortality (Table 2). This was also found in the trials including peri-operative and trauma patients.

\section{Blinding}

Only three studies $(12 \%)$ randomised patients with adequate blinding. In these studies, all including patients peri-operatively the significant effect on mortality was similar compared to a significant reduction in the studies with inadequate blinding (Table 2). 


\section{Cross-over}

In the studies in which cross-over between the treatment arms were adequately controlled, treatment effect was not significantly improved compared to the studies with significant cross-over, in which a significant improvement was found Table 4). In the studies optimising peri-operatively and directly post-traumatically both the studies with and without significant cross-over had a significant lower mortality when haemodynamic optimisation strategies were used.

\section{Supranormal oxygen delivery as goal of treatment}

In the peri-operative studies the subgroup analysis including individual studies $(3,17-19,27,36-38)$ using the original criteria as proposed by Shoemaker (including 1053 patients) a relative risk ratio of $0.46(0.35-0.62)$ was found (Table 2). In these studies 5 patients $(95 \% \mathrm{Cl} 4-7)$ needed to be treated to save 1 life. The quality control score of this subgroup was 9.1. Studies using treatment goals other than supranormal oxygen dellivery in peri-operative patients had a similar effect on mortality; relative risk ratio $0.39(0.20-0.76)$.

In the studies including patients with sepsis and organ failure neither the use of supranormal oxygen delivery $(20-26,40)$ nor other specified treatment goals showed a reduction in mortality; relative risk ratios were $1.00(95 \% \mathrm{Cl} 0.90-1.11)$ and 0.85 (95\% Cl 0.55-1.31), respectively (Table 2).

For all studies combined, only including patients optimised using criteria proposed by Shoemaker $(n=2091)$ the relative risk ratio was $0.73(95 \% \mathrm{Cl} 0.58-0.93)$. This significant effect was not found in the patient group not using the supranormal oxygen delivery as end-goal; relative risk ratio 0.69 (95\% Cl 0.45-1.05) (Table 2).

\section{Discussion}

This meta-analysis, in which we conducted a systematic search, selection, and quality assessment of the literature, suggests that haemodynamic optimisation techniques when used in peri-operative and trauma patients can improve survival. Overall, patients with sepsis and overt organ failure do not benefit from this method. All studies combined suggest that haemodynamic optimisation improves mortallity, but is mainly based upon the results of the peri-operative studies. Since our findings may have important implications in clinincal surgical practice, we also related our results to the quality of the individual trials, which, however, was moderate and seemed to have influenced the outcome of the individual trials.

The use of haemodynamic optimisation as a therapy to improve outcome is controversial. The regimen was originally designed to optimise the haemodynamic status in high-risk surgical patients. The initial studies found an improved outcome. A large number of studies including different patient populations and optimisation techniques were conducted subsequently. In a considerable number of these 
studies no improved outcome was observed $(21,23,29)$. Moreover, in one study mortality increased in the optimised patients (27). The meta-analysis by Heyland et al. reporting the first seven studies published at that time, showed overall no benefit from maximizing oxygen delivery aimed to improve outcome (4).

It was suggested, in the discussion of the meta-analyses by Heyland et al., that the outcome results from the studies with late interventions should be separated from the results of the studies with early interventions (4). Recent publications have shown a pathophysiological basis for this difference. Early in the disease process of the systemic inflammatory response syndrome peripheral defects in oxygen delivery, based on decreased flow, hypoxia or hypovolaemia can be prevented or overcome. In contrast, persistent defects in oxygen delivery to the tissues during decreased fllow or hypovolaemia may alter vascular and cellular metabolism. These defects in cellular oxygenation become irreversible due to mitochondrial damage and when occurring in the endothelium lead to vascular hyporeactivity or 'vasoplegia'. This results in an impaired perfusion and organ failure. Moreover, organ function is less likely to recover due to the relative insensitivity of patients with multiple organ failure to the optimisation techniques $(7,43,44)$. The study by Rivers et al., in which early optimisation of the haemodynamics led to a reduced mortality, even in patients with early septic shock, emphasizes this point (31). Indeed, two recent meta-analyses confirmed that improvement of outcome can be achieved in patients optimised before organ failure has occurred. In the study by Boyd et al. early intervention studies showed an improved survival compared to the late intervention studies (8). The meta-analysis by Kern and Shoemaker made a division between early optimisation studies ( 8 to $12 \mathrm{~h}$ post-operatively or before organ failure developed) versus late optimisation studies (after organ failure developed) (9). They also found a significant reduction in mortality in the early optimisation studies. However, since vigorous attempts should be made to minimise bias in performing randomised controlled trials, a meta-analysis should include these confounders into the analysis. Both meta-analyses, however, did not present a formal quality assessment $(8,9)$. Our findings suggest that the methodological quality of all studies combined may have significantly influenced the effectiveness of the haemodynamic optimisation strategies. Studies with a quality score $>10$ failed to show an improved survival. Moreover, the studies complying to important individual quality factors, such as blinding and control of crossover, failed to show significant improvement in mortality. On the other hand no significant correlation coefficient was present between quality score and outcome in both high-risk surgical patients and patients with sepsis and organ failure. The use of a single quality score may influence this outcome. Juni et al. suggested that relevant methodological aspects should be assessed separately and that their influence on effect sizes should be taken into account (13). Most of the items mentioned by Juni et al. were included into the scoring system used by Heyland et al. (4) and were used in our meta-analysis. In addition, mortality as end-point was included as separate quality factor, since not all relevant trials were designed and powered to a reduced mortality. This quality factor did not influence the outcome of the meta-analysis. Moroever, the individual quality factors seemed 
to have less relevance on outcome in studies including peri-operative and posttraumatic patients.

Several studies have been published using haemodynamic optimisation strategies, but not using the specific goals as defined by Shoemaker et al. (3). These studies were not included in the meta-analysis by Kern et al. (9), which may have introduced selection-bias. In our meta-analysis these studies were included, since we wanted to analyse whether attainment to the criteria as defined by Shoemaker et al. (3) had an additional beneficial or detrimental influence on mortality over using other goals of haemodynamic optimisation. We conclude that in perioperative patients the use of the criteria defined by Shoemaker et al. (3) did not reduce mortality further. It has been suggested that in these other studies only normalisation of the haemodynamics is obtained without real optimisation of haemodynamic profiles. However, for example, in the study by Mythen et al. (34) and Sinclair et al. (35) haemodynamic optimisation was achieved using optimal stroke volume as goal of therapy. Apparently, the exact goal for obtaining supranormal haemodynamics is less important, than the fact that peri-operative patients should be hsemodynamically optimised. The importance of titrate haemodynamic therapeutic interventions to the individual needs of a patient has been emphasised before (45).

In conclusion, the results of this meta-analysis suggest that haemodynamic optimisation reduces mortality in high-risk surgical and traumatised patients, although all patients seemed to benefit from haemodynamic optimisation strategies. However, concerns remain with respect to the influence of the methodological quality of the individual trials. Further large scale, multicenter, blinded studlies are necessary. 


\section{REFERENCES}

1. Wo CC, Shoemaker WC, Appel PL, Bishop MH, Kram HB, Hardin E. Unreliability of blood pressure and heart rate for evaluation of circulatory stability in emergency resuscitation and critical illness. Crit Care Med 1993; 21:218-223.

2. Bishop MH, Shoemaker WC, Appel PL, et al. Relationship between supranormal circulatory values, time delays, and oulcome in severely traumatized patients. Crit Care Med 1993;2111):56-63.

3. Shoemaker WC, Appel PL, Kram HB, Waxman MD, Lee T-S. Prospective trial of supranormal values of survivors as therapeutic goals in high-risk surgical patients. Chest 1988,94(6):1176-1186.

4. Heyland DK, Cook DJ, King D, Kernerman P, Brun-Buisson C. Maximizing axygen delivery in critically ill patients: a methodological appraisal of the evidence. Crit Care Med 1996;24(3):517-524.

5. Poeze M, Greve IWM, Ramsay G. Oxygen delivery in septic shock. Chest 1999;1 16(4):1145 (lietterl.

6. Poeze $M$, Greve JWM, Ramsay $G$. Coal-oriented haemodynamic therapy: a plea for a closer look at using peri-operative oxygen transport optimisation. Intensive Care Med 2000;26:635-637.

7. Sibbald WI, Fox G, Martin C. Abnormalities of vascular reactivity in the sepsis syndrome. Chest 1991;100(3):155S-159S.

8. Boyd $O_{r}$ Hayes $M$. The oxygen trail: the goal. Br Med Bull 1999;55(1):125-139.

9. Kern JW, Shoemaker WC. Meta-analysis of hemodynamic optimisation in high-risk patients. Crit Care Med 2002,30:1686-1692.

10. de Bie RA. Methodology of systematic reviews: an introduction. Phys Ther Rev 1996;1:47-51.

11. Boissel JP. Standards for reporting clinical trials. 1993; 28:195-197.

12. Chalmers TC, Smith $H$, Blackburn B, et al. A method for assessing the quality of a randomized control trial. Control.Clin. Trials 1981;2(1):31-49.

13. Juni $P$, Witschi $A, B$ loch $R$, Egger $M$. the hazards od scoring the quality of clinical trials for metaanalysis. JAMA 1999;282:1054-1060.

14. DerSimonian R, Laird N. Meta-analysis in clinical trials. Control Clin Trials 1986;7(3):177-188*

15. Berlauk $\Downarrow F_{r}$ Abrams JH, Gilmour IJ, $O^{\prime}$ Connor $S R$, Knighton DR, Cerra FB. Preoperative optimization of cardiovascular hemodynamics improves outcome in peripheral vascular surgery. A prospective, randomized clinical trial. Ann Surg 1991;214(3):289-297.

16. Bender IS $S_{r}$ mith-Meek MA, Jones CE. Routine pulmonary artery catheterization does not reduce morbidity and mortality of elective vascular surgery: results of a prospective, randomized trial. Ann:Surg 1997;226(3):229-236.

17. Ziegler DW, Wright $\| G$, Choban PS, Flancbaum L. A prospective randomized trial of preoperative "optimization" of cardiac function in patients undergoing elective peripheral vascular surgery. Surgery 1997; 122(3):584-592.

18. Valentine RJ, Duke ML, Inman MH, et al. Effectiveness of pulmonary artery catheters in aortic surgery: a randomized trial. J Vasc. Surg 1998;27(2);203-211.

19. Ueno $S$, Tanabe $G_{t}$ Yamada $H$, et al. Response of patients with cirrhosis who have undergone partial hepatectomy to treatment aimed at achieving supranormal oxygen delivery and consumption. Surgery 1998:123(3):278-286.

20. Wilson I Woods I, Fawcett J, et al. Reducing the risk of major elective surgery: randomised controlled trial of preoperative optimisation of oxygen delivery. BMJ 1999;318(7191):10991103.

21. Velimahos $C$, , Demetrilades $D$, Shoemaker $W C$, et al. Endpoints of resuscitation of critically injured patients: normal or supranormal? A prospective randomized trial. Ann Surg 2000;232(3):409-418. 
22. Takala J, Meier Hellmann $A$, Eddleston $J M_{\text {, }}$ et al. for the European Multicenter Study Group on Dopexamine in Major Abdominal Surgery. Effect of dopexamine on outcome after major abdominal surgery: a prospective, randomized, controlled multi-center study. Crit Care Med 1998;28(10):3417-3423.

23. Tuchschmidt J, Fried J Astiz M, Rackow E. Elevation of cardiac output and oxygen delivery improves outcome in septic shock. Chest 1992;102(1):216-220.

24. Yu $M$, Levy MM, Smith $P$, Takiguchi SA, Miyasaki A, Myers SA. Effect of maximizing oxygen delivery on morbidity and mortality rates in critically ill patients: a prospective, randomized, controlled study. Crit Care Med 1993;21(6):830-838.

25. Yu $M_{*}$ Takanishi $D, M y e r s ~ S A$, et al. Frequency of mortality and myocardial infarction during maximizing oxygen delivery: a prospective, randomized trial. Crit Care Med 1995;23(6):1025-1032.

26. Yu M, Burchell S, Hasaniya NW, Takanishi DM, Myers SA, Takiguchi SA. Relationship of mortality to increasing oxygen delivery in patients $>$ or $=50$ years of age: a prospective, randomized trial. Crit Care Med 1998;26(6):1011-1019.

27. Hayes MA, Timmins AC, Yau EHS, Palazzo M, Hinds C, Watson D. Elevation of systemic oxygen delivery in the treatment of critically ill patients. New Eng J Med 1994,330(24):1777 7-1722.

28. Alia I, Esteban A, Gordo $F$, et al. A randomized and controlled trial of the effect of treatment aimed at maximizing oxygen delivery in patients with severe sepsis or septic shock. Chest 1999;115(2):453-461.

29. Gattinoni $L$, Brazzi L, Pelosi $P$, et al., for the $\mathrm{SvO}_{2}$ Collaborative Group. A trial of goal-oriented hemodynamic therapy in critically ill patients. New Eng I Med 1995;333(16):1025-1032.

30. Lobo SM, Salgado PF, Castillo VG, et al. Effects of maximizing oxygen delivery on morbidity and mortality in high-risk surgical patients. Crit Care Med 2000;28(10):3396-3404.

31. Rivers E, Nguyen B, Havstad $S_{v}$ et al., Early Goal-Directed Therapy Collaborative Group. Early goal-directed therapy in the treatment of severe sepsis and septic shock. New Eng J Med 2001;345(19):1368-1377.

32. Polonen $P$, Ruokonen $E$, Hippelainen $M$, Poyhonen $M$, Takala J. A prospective, randomized study of goal-oriented hemodynamic therapy in cardiac surgical patients. Anesth Analg 2000;90(5):1052-1059.

33. Schultz RI, Whitfield GF, LaMura IJ, Raciti A, Krishnamurthy S. The role of physiologic monitoring in patients with fractures of the hip. I Trauma 1985;25(4):309-316.

34. Mythen MG, Webb AR. Perioperative plasma volume expansion reduces the incidence of gut mucosal hypoperfusion during cardiac surgery. Arch Surg 1995;130(4):423-429.

35. Sinclair $S$, James $S$, Singer $M$. Intraoperative intravascular volume optimisation and length of hospital stay after repair of proximal femoral fracture: randomised controlled trial. BMI 1997;315(7113):909-912.

36. Boldt J. Papsdorf $M$, Piper S, Padberg W. Hempelmann G. Influence of dopexamine hydrochloride on haemodynamics and regulators of circulation in patients undergoing major abdominal surgery. Acta Anaesthesiol Scand 1998;42(8):9411.947.

37. Garrison RN, Wilson MA, Matheson PJ, Spain DA. Preoperative saline loading improves outcome after elective, noncardiac surgical procedures. Am Surg 1996;62(3):223-231.

38. Blow O, Magliore L, Claridge JA, Butler K, Young J5. The golden hour and the silver day: detection and correction of occult hypoperfusion within 24 hours improves outcome from major trauma. J Trauma 1999;47(5):964-969. 
39. Fleming $A$, Bishop $M_{f}$ Sthoemaker $W_{s}$ et al. Prospective trial of supranormal values as goals of resuscitation in severe trauma. Arch Surg 1992;127:1175-1181.

40. Boyd $O$, Grounds RM, Beninett ED. A randomized clinical triall of the effect of deliberate perioperative increase of oxygen delivery on mortality in high- risk surgical patients. JAMA 1993;270(22):2699-2707.

41. Bishop $\mathrm{MH}_{r}$ Shoemaker $\mathrm{WC}_{\mathrm{r}}$ Appel $\mathrm{PL}$, et al. Prospective, randomized trial of survivor values. of cardiac index, oxygen delivery, and oxygen consumption as resuscitation endpoints in severe trauma. I Trauma 1995;38(5):780-787.

42. Durham RM, Neunaber K, Mazuski IE, Shapiro MJ, Baue AE. The use of axygen consumption and delivery as endpoints for resuscitation in critically ill patients. I Trauma 1996;41(1):32-39.

43. Hollenberg SM, Cuninion RE. Endothelial and vascular smooth muscle function in sepsis. / Crit Care 1994;9(4):262-280.

44. Abidd $O$, Akca $S_{x}$ Haji-Michael $P$, Wincent H-L. Strong vasopressor support may be futile in the intensive care unit patient with multiple organ failure. Crit Care Med 2000;28(4):947-949.

45. Dabrowski GP, Steinberg SM, Ferrara II, Flint LM. A critical assessment of endpoints of shock resuscitation. Surg Clin North Am 2002;80:825-844. 


\section{CHAPTER 7}

Moniloring global volume-related

haemodynamic or regional parameters after initial resuscitation: which is a better predictor of outcome in critically ill septic patients? 


\section{ABSTRACT}

Objective. Regional parameters of organ dysfunction are thought to be better monitoring parameters than global pressure-related haemodynamic parameters. Whether a difference exists between regional or global volume-related parameters in critically ill patients before and after resuscitation is unknown.

Design. Prospective dinical study.

Seting. University-affilated mixed Intensive care unit.

Patients. 28 critically ill patients were prospectively stratified into groups +/hypotension.

Interventions. Using standardised resuscitation haemodynamic optimisation was targeted at mean arterial pressure (MAP), heart rate (HR), wedge pressure (PCWP), cardiac output $(\mathrm{CO})$, systemic vascular resistance, and urine output. Primary outcome variable was in-hospital mortality.

Measurements and results: During resuscitation global volume-related haemodynamic parameters were measured simultaneously and compared with regional parameters. At admission MAP, central venous pressure (CVP), gastric intramucosal $\mathrm{pH}$ ( $\mathrm{pHi}$ ), clearance of indocyanine green (ICG) from blood (CBI) and plasma (CPI), arterial $\mathrm{pH}$, and left ventricular stroke work index were significantly different between patients with and without hypotension, although no parameter was superior as predictor of outcome. Resuscitation increased MAP, CVP, oxygen dellivery, and right-ventricular ejection fraction significantly. After stabilisation significant differences persisted between the two groups with

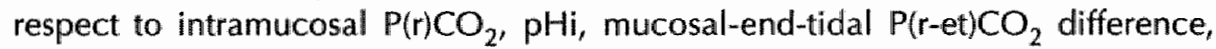
$\mathrm{CBI}, \mathrm{CPI}$, plasma disappearance rate $(\mathrm{PDR})$, and right ventricular end-diastolic volume (RVEDVI). Patients who subsequently died had a significant lower $\mathrm{pHi}$, higher $\mathrm{PrCO}_{2}, \mathrm{Pr}$-etCO $\mathrm{C}_{2}$, and lower CBI, CPl, PDR, and RVEDVI, of which the pHi, Pr-etCO $\mathrm{CO}_{2}$, and $\mathrm{CBI}$ were the most important predictors of outcome.

Conclusions: Initial resuscitation of critically ill patients with shock does not require monitoring of regionall parameters. However, after stabilisation regional parameters were the best predictors of outcome. Further resuscitation efforts in this phase should be aimed at influencing these regional parameters. 
The ability to recognise the features of shock is crucial in the management of critically ill patients. In trauma patients failure to recognise the presence or the extent of shock has been identified as an important contributory factor leading to a considerable number of 'preventable' deaths (1). During therapy of shock the standard resuscitation is aimed at correcting global pressure-related parameters. However, correcting these parameters produces a varying outcome in critically ill patients. There are several possible reasons for this difference in outcome. The recognition of shock can be hampered by the compensatory capacities of the patients. A patient may be in shock despite a normal heart rate and blood pressure. Moreover, the therapy used to treat shock can normalise parameters by which shock is assessed clinically, although a defective tissue oxygenation may still exist (2-4). Global pressure-related parameters may be too inaccurate to detect these abnormalities.

Therefore, there is a need for other indicators of tissue oxygenation in patients with shock, which are more sensitive and specific than the global pressure-related parameters. Two approaches have been introduced, which may be able to detect occult hypovolaemia.

It has been suggested that measuring regional parameters of splanchnic perfusion is a better predictor of the presence of uncompensated shock than markers of global perfusion $(5,6)$. Splanchnic perfusion seems to play an important role during shock and resuscitation. Recent studies suggest the presence of a disturbed splanchnic circulation in apparently compensated shock (7-9). Critically ill patients with a persistent inadequate splanchnic perfusion are at increased risk of developing multiple organ failure during their intensive care stay $(10,11)$.

Another possibility is measuring the global volume-related parameters (such as extravascular lung water volume or intrathoracic blood volume) instead of pressure-related parameters. A considerable amount of studies indicate the lack of relation between the degree of hypovolaemia and the pressure-related parameters $(12,13)$. Instead, the use of volume-related global haemodynamic parameters is thought to indicate the presence of hypovolemia (14-16).

Keeping these observations in mind, we carried out a prospective study in which critically ill septic patients with or without overt clinical shock underwent a standardised resuscitation in order to normalise the global pressure-related haemodynamics. The response of both the global volume-related haemodynamic and the regional parameters to this resuscitation were evaluated with respect to the predictive value for mortality. Our hypothesis was that resuscitation on the intensive care unit could be aimed at the global pressure-related parameters, but after normalisation optimal resuscitation can only be achieved using regional parameters. 


\section{PATIENTS AND METHODS}

\section{Study design}

This study was conducted as a stratified prospective evaluation of the predictive value of global pressure- and volume-related haemodynamic and regional parameters of splanchnic perfusion during the intensive care resuscitation of critically ill septic patients. The in-hospital mortality was used as primary end-point of this study.

In the study protocol (Figure 1) it was prospectively defined that the effects of the resuscitation should be analysed after pre-stratifying the study group into subgroups with and without hypotension at admission to the ICU, since this was thought to alter the haemodynamic supportive efforts considerably. The presence of hypotension was defined as a systolic blood pressure $<90 \mathrm{mmHg}$ or a sustained drop of $\approx 40 \mathrm{mmHg}$ from known (chronic) baseline values.

Patients fulfilling the inclusion criteria were included during a time-period of 2 years from a mixed ICU in a university hospital. The Institutional Review Board of the hospital approved this study. Written informed consent was obtained from the next of kin of each patient, since all patients were ventilated and sedated at the time of inclusion to the study.

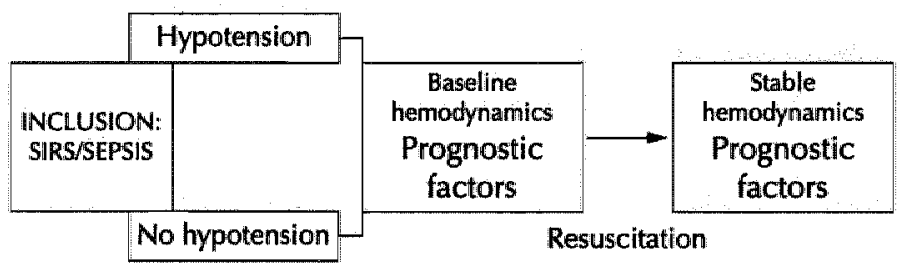

Schenatic depiction of the prospective study comparing global haemodynamic and hepatosplanchnic parameters. After indusion patients were a priori divided into a subgroup with and a subgroup without sepsis-induced hypotension. At admission the predictive value of the haemodynamic parameters were assessed. Subsequently, patients were resuscitated according to global pressure-related hamomodynamic parameters. After stabilisation of these parameters was acquired, predlictive value of these haemodynamic parameters were assessed again.

Figure 1 Illustration of the study

\section{Study patients}

Critically ill patients with sepsis as defined by the ACCP/SCCM consensus conference criteria were included (17). In the presence of clinically suspected or proven infection patients were defined as having sepsis. A clinical suspicion of infection was defined as the presence of abnormalities on chest $\mathrm{X}$-ray consistent 
with pulmonary infection, peritonitis confirmed at surgery or clinical examination, or Gram stain positive for infection. All patients were ventilated. Patients were included when APACHE-II score $>12$.

Patients with sepsis-induced hypotension or severe sepsis who were expected to succumb within the first $24 \mathrm{hrs}$ were not included. Also, patients on dialysis were excluded from study participation.

\section{Measurements of systemic and regional parameters}

After inclusion patients received a 3F thermistor-tipped fibre optic catheter (PV 2024, Pulsion Medical Systems, Munich, Germany) via a $4 \mathrm{~F}$ introducing sheath (Arrow, Reading, USA) in the femoral artery placed into the descending aorta. After this the catheter was connected to a bedside monitor (COLD Z-021, Pulsion Medical Systems, Munich, Germany). Moreover, a pulmonary artery thermodilution catheter (7.5F, Baxter, USA) was introduced and also attached to the COLD bedside monitor. A gastric tonometry catheter (14F, Datex Ohmeda, Finland) was introduced for measurement of intramucosal $\mathrm{PrCO}_{2}$ using the gas-automated capnograph (Tonocap TC-200, Datex-Ohmeda, Finland). Correct placement of these catheters was determined by radiographic investigations. The gastric mucosal $\mathrm{pH}$ (pHi) was calculated using the standard Henderson-Hasselbach formula (18). At admission to the ICU several parameters were obtained in order to evaluate disturbances in haemodynamics and organ function. These parameters could be divided into four groups.

The routine global haemodynamic parameters: using central arterial and a pulmonary artery flotation catheter the mean arterial pressure (MAP), heart rate (HR), pulmonary capillary wedge pressure (PCWP), and cardiac index (Cl) were measured. Mixed venous and arterial blood gasses were used to measure lactate and to calculate the oxygen delivery $\left(\mathrm{DO}_{2}\right)$ and oxygen consumption $\left(\mathrm{VO}_{2}\right)$ and were drawn simultaneously with the thermodilution measurement. From the global haemodynamic parameters the systemic vascular resistance index (SVRI) was calculated. Urine output was measured using a Foley urinary catheter.

The double dilution measurements were performed using a $10 \mathrm{ml}$ cold bolus infusion of $1 \mathrm{mg} / \mathrm{kg}$ indocyanine green (ICG) in saline. The principle of these measurements is based upon the distribution of an indicator with the greatest volume available as has been described in detail in (18). The following parameters were determined: right heart- and right ventricular end-diastolic volume index (RHEDVI and RVEDVI), right ventricular ejection fraction, end-systolic volume, and stroke work index (RVEF, RVESVI, RVSWI), left heart end-diastolic volume index (LHEDVI), global end-diastolic volume index (GEDVI), extra vascular lung water index (EVLWI), intrathoracic blood volume index, total blood volume and pulmonary blood volume index (ITBVI, TBVI, and PBVI).

The indocyanine green (ICG)-clearance measurements ICG blood clearance (CBI), ICG plasma clearance $(\mathrm{CPI})$, and plasma disappearance rate of dye (PDR) were performed using the bolus infusion method of ICG. The PDR rate is determined from the linear elimination curve of ICG through the liver. The $C B I$ and $C P I$ are 
calculated by multiplying the PDR with the TBV (and (1-haematocrit) for the CPI), The gastric tonometry measurements $\mathrm{PrCO}_{2}$, mucosal-end tidal $\mathrm{pCO}_{2}$-gap ( $\mathrm{Pr}$-etCO $\mathrm{CO}_{2}$-gap), and mucosal-arterial $\mathrm{pCO}_{2}$ gap $\left(\mathrm{Pr}^{-\mathrm{aCO}} \mathrm{CO}_{2}\right.$ gap) were measured with a 10-minute interval using the gas-automated capnography (Tonocap, Datex-Ohmeda). The $\mathrm{pCO}_{2}$ and $\mathrm{pH}$ values from the blood gases were corrected for the central blood temperature measurements, using the formulas as provided by the manufacturer (ABL 100, Radiometer, Kopenhagen).

\section{Resuscitation protocol}

Resuscitation was aimed at improving global (pressure-related) haemodynamics as judged by the intensivist on call: in general, circulatory support was titrated to increase the MAP $>70 \mathrm{mmHg}$, systemic vascular resistance $>1,100 \mathrm{dyn} / \mathrm{s} / \mathrm{cm}^{5} / \mathrm{m}^{2}$, $\mathrm{Cl}>2.5 \mathrm{l} / \mathrm{min} / \mathrm{m}^{2}$ and urine output $>0.5 \mathrm{ml} / \mathrm{kg} / \mathrm{h}$. If these criteria were not met and PCWP $<18 \mathrm{mmHg}$, a fluid challenge of $500 \mathrm{cc}$ or $1000 \mathrm{cc}$ (depending on blood pressure) saline $0.9 \%$ and/or Gelofusine (Braun, Melsungen, Germany) was given. If this caused an increase in $\mathrm{Cl}$ and/or MAP $>10 \%$ with the PCWP remaining below $18 \mathrm{mmHg}$, another fluid challenge could be given. If the increase in $\mathrm{Cl}$ or MAP was $<10 \%$, then fluid challenging was stopped, regardless of PCWP. After fluid challenging, dobutamine and/or noradrenaline $(19,20)$ were given guided by the effects on $\mathrm{Cl}$ and MAP, aiming at a $\mathrm{Cl}>2.5 \mathrm{l} / \mathrm{min} / \mathrm{m}^{2}$ and a MAP $>70 \mathrm{mmHg}$. If $P C W P$ was $>18 \mathrm{mmHg}$ dobutamine was given primarily. Whenever hematocrit was $<0.3$, a transfusion of 2 units of packed red cells was allowed. The intensivists were blinded for the results of the regional tonometric and ICG-dilution parameters.

A stable haemodynamic status was defined by the intensivist using the following guidelines; MAP > $70 \mathrm{mmHg}$, or a stable blood pressure (variation in MAP < $10 \mathrm{mmHg}, H R<30 \%$ ) during two hours without the necessity of increasing any vasopressor or inotropic therapy or without the need of fluid administration in order to treat a decrease in MAP $>10 \mathrm{mmHg}$ or a change in heart rate $>30 \%$. This definition was used to divide the study period into two periods: a time-period from admission and a time-period after stabilisation. Although the periods before and after stabilisation were seen as two distinct time-periods, in practice the same goals for resuscitation were used as a continuum in the primary and secondary phase. After stabilisation was achieved the above-mentioned measurements were repeated.

None of the patients were enterally fed during the study period. $\mathrm{H}_{2}$-blockers were given routinely, using ranitidine. Antibiotics were given blindly or directed at idlentified organisms.

\section{Follow-up}

Patients' follow-up was continued during the remainder of the ICU admission. After discharge from the ICU, patients were monitored for 28 days or until final hospital discharge, whichever was longer, for morbidity and mortality. 


\section{Statistics}

Data were analysed using the SPSS (version 7.5) software program. Data from patient characteristics were assessed using Student t-test and chi-square test. Admission variables were assessed using one-way ANOVA. Data during the resuscitation period were compared using the multiple-way ANOVA test, which produced three $p$-values for each analysis: the first $p$-value $\left(P_{T}\right)$ indicates for all patients combined whether during the resucitation-period changes were found, the second $p$-value $\left(P_{G}\right)$ indicates whether overall variables were significant between the groups with and without hypotension analysed and the third $\mathrm{p}$-value $\left(\mathrm{P}_{\mathrm{TxC}}\right)$ indicates whether during this time-period significantly different changes were noted between the two groups analysed (multiple-way interactions).

To detect the most important predictors of mortality, haemodynamic variables were analysed using a logistic non-parametric multiple regression model (backward conditional). The validity of the different tests was evaluated by using a method for comparing areas under the receiver operator characteristic (ROC) curves, as previously described in detail $(21,22)$. AUCs were calculated for the parameters at admission and after stabilisation. For the parameters after stabilisation the odds ratio were calculated using the optimal cut-off point derived from the ROC-curves. The optimal cut-off value for predicting mortality was calculated as that point with the greatest combined sensitivity and specificity. For all analyses a $p$-value $<0.05$ was considered statistically significant.

\section{RESULTS}

\section{Patients}

In total 28 patients with severe sepsis or septic shock were included into the analysis and monitored prospectively. The presence of infection was suspected in 17 patients. The mean APACHE-II score at admission was 17. The median time between the start of the symptoms and admission to the ICU was $18 \mathrm{hrs}$. Of the 28 patients, 12 patients were admitted in shack (Table 1). There were no differences between the two groups with respect to age, gender, duration of complaints before inclusion, number of patients with sepsis, and APACHE - 11 score. MOF-score after $24 \mathrm{hrs}$ was significantly higher in patients with hypotension at admission. Mortality was significantly higher in the patients with $(75.0 \%)$ than without $(31.3 \%)$ shock $(p=0.03)$. 
Table 1 Patient demographics

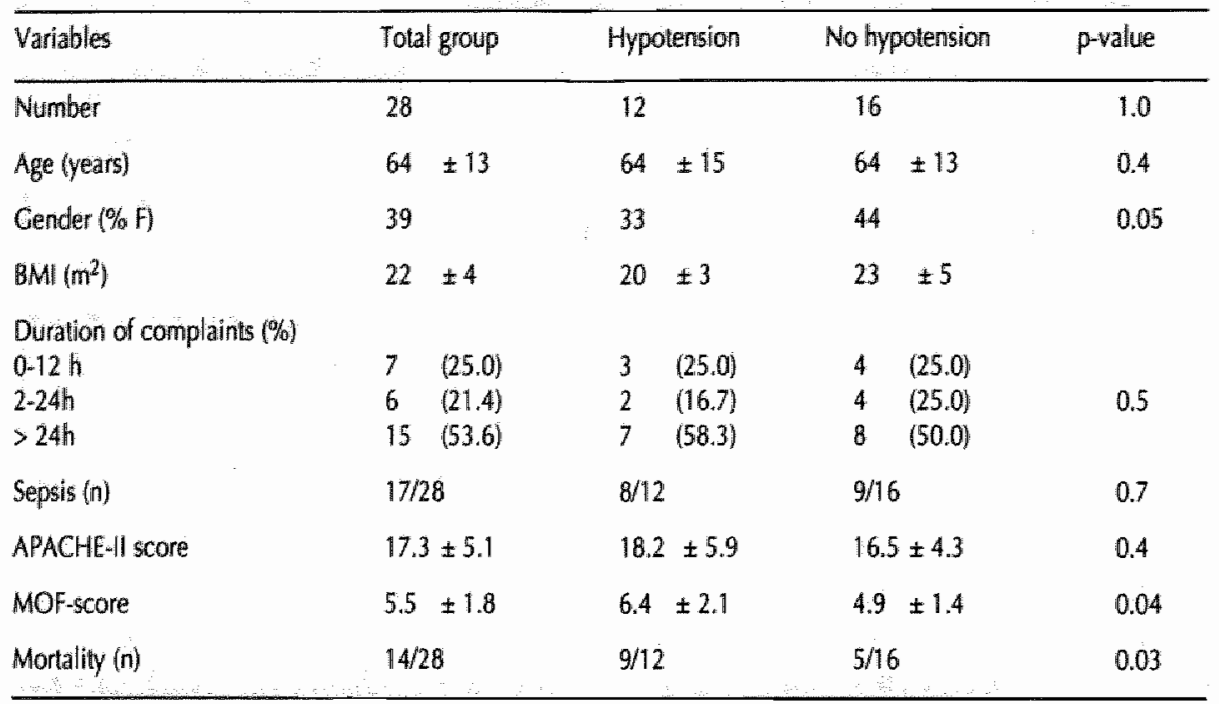

Data are presented as mean \pm standard deviation or as percentages. The p-value indicates the significance level for the comparison between patients with and without hypotension at admission. \% $F$, percentage fermales; BMil; body mass index; MOF, multiple organ failure.

Table 2 Global and regional haemodynamic changes during resuscitation in patients with and without hypotension

\begin{tabular}{|c|c|c|c|c|c|c|c|c|}
\hline \multicolumn{2}{|l|}{ Parameters } & \multicolumn{2}{|c|}{ At admission } & \multicolumn{2}{|c|}{ After stabilisation } & \multicolumn{3}{|c|}{ p-values } \\
\hline \multicolumn{9}{|c|}{ Global pressure-related parameters } \\
\hline \multirow[t]{2}{*}{ MAP } & hypotension & 64 & \pm 5 & 76 & \pm 9 & 0.001 & $<0.0001$ & 0.03 \\
\hline & no hypolension & 81 & \pm 11 & 84 & \pm 13 & & & \\
\hline \multirow[t]{2}{*}{ CVP } & hypotension & 12.2 & \pm 3.9 & 15.6 & \pm 4.0 & 0.04 & $<0.0001$ & 0.03 \\
\hline & no hypotension & 10.5 & \pm 3.7 & 10.5 & \pm 3.6 & & & \\
\hline \multirow[t]{2}{*}{ DO21 } & hypotension & 545 & \pm 111 & 672 & \pm 238 & 0.04 & 0.1 & 0.4 \\
\hline & no hypotension & 648 & \pm 195 & 698 & \pm 254 & & & \\
\hline \multirow[t]{2}{*}{ SVRI } & hypotension & 1070 & \pm 444 & 988 & \pm 233 & 0.7 & 0.001 & 0.6 \\
\hline & no hypotension & 1355 & \pm 456 & 1370 & \pm 609 & & & \\
\hline \multirow[t]{2}{*}{ Lactate } & |rypotension & 2.1 & $\pm 1,9$ & 2.0 & \pm 1.7 & 0.2 & 0,003 & 0.5 \\
\hline & no hypotension & 1.5 & \pm 1.1 & 1.0 & \pm 0.5 & & & \\
\hline \multirow[t]{2}{*}{ Base deficit } & hypotension & -5.4 & \pm 4.3 & $-6,4$ & \pm 6.3 & 0.3 & 0.004 & 0.9 \\
\hline & no hypotension & -1.7 & \pm 7.3 & -3.0 & \pm 6.1 & & & \\
\hline
\end{tabular}




\begin{tabular}{|c|c|c|c|c|c|c|c|c|}
\hline \multirow[t]{2}{*}{ Parameters } & & \multicolumn{2}{|c|}{ At admission } & \multicolumn{2}{|c|}{ After stabilisation } & \multicolumn{3}{|c|}{ p-walues } \\
\hline & & & & & & $P_{T}$ & $P_{G}$ & $P_{T \times C}$ \\
\hline \multicolumn{9}{|c|}{ Clobal volume-related parameters } \\
\hline \multirow[t]{2}{*}{ TBMI } & hypotension & 3312 & \pm 857 & 3464 & \pm 747 & 0.8 & 0.009 & 0.3 \\
\hline & no hypotension & 4032 & \pm 1031 & 3801 & \pm 929 & & & \\
\hline \multirow[t]{2}{*}{ RHEDVI } & hypotension & 385 & \pm 114 & 401 & \pm 171 & 0.5 & 0.02 & 0.3 \\
\hline & no hypotension & 521 & \pm 223 & 448 & \pm 194 & & & \\
\hline \multirow[t]{2}{*}{ RVEDVI } & hypotension & 163 & \pm 7 & 158 & \pm 48 & 0.3 & 0.02 & 0.5 \\
\hline & no hypotension & 194 & \pm 61 & 176 & \pm 33 & & & \\
\hline \multirow[t]{2}{*}{ RVSWI } & hypotension & 8.7 & \pm 3.6 & 9.3 & \pm 4.3 & 0.2 & 0.009 & 0.5 \\
\hline & mo hypotension & 10.2 & \pm 3.9 & 11.9 & \pm 4.0 & & & \\
\hline \multirow[t]{2}{*}{ LWSWI } & hypotension & 28.0 & \pm 12.3 & 36.6 & \pm 13.0 & 0.06 & $<0.0001$ & 0.4 \\
\hline & no hypotemsion & 42.3 & \pm 15.6 & 45.4 & \pm 17.7 & & & \\
\hline \multirow[t]{2}{*}{ RVEF } & hypotension & 33.7 & \pm 8.4 & 41.3 & \pm 7.0 & 0.03 & 0.01 & 0.7 \\
\hline & no hypotension & 33.5 & \pm 6.7 & $43 ; 9$ & \pm 8.1 & & & \\
\hline \multicolumn{9}{|c|}{ Regional parameters } \\
\hline \multirow[t]{2}{*}{$\mathrm{pHi}$} & hypotension & 7.25 & \pm 0.12 & 7.21 & \pm 0.17 & 0.5 & 0.005 & 0.3 \\
\hline & no hypotension & 7.36 & \pm 0.25 & 7.31 & \pm 0.19 & & & \\
\hline \multirow[t]{2}{*}{$\mathrm{PrCO} 2$} & hypotension & 6.6 & \pm 2.5 & 7.3 & \pm 1.7 & 0.3 & 0.03 & 0.5 \\
\hline & no hypotension & 5.9 & \pm 1.5 & 6.1 & \pm 1.3 & & & \\
\hline \multirow[t]{2}{*}{ PretcO2 } & hypotension & 2.5 & \pm 0.4 & 3.4 & \pm 0.7 & 0.4 & 0.02 & 0.2 \\
\hline & no hypotension & 2.2 & \pm 0.2 & 1.9 & \pm 0.1 & & & \\
\hline \multirow[t]{2}{*}{ CBI } & hypotension & 464 & \pm 310 & 407 & \pm 221 & 0.4 & $<0.0001$ & 0.9 \\
\hline & no hypotension & 862 & \pm 408 & 795 & \pm 343 & & & \\
\hline \multirow[t]{2}{*}{$\mathrm{CPI}$} & hypotension & 338 & \pm 240 & 328 & \pm 193 & 0.6 & $<0.0001$ & 0.7 \\
\hline & no hypotension & 598 & \pm 305 & 542 & \pm 244 & & & \\
\hline \multirow[t]{2}{*}{ PDR } & hypotensian & 14.6 & \pm 8.8 & 11.8 & \pm 6.4 & 0.4 & $<0.0001$ & 0.5 \\
\hline & no hypotension & 20.5 & \pm 9.4 & 20.2 & \pm 7.8 & & & \\
\hline \multirow[t]{2}{*}{ Urine ouput } & hypotension & 0.7 & \pm 0.9 & 1.0 & \pm 1.0 & 0.08 & 0.001 & 0.3 \\
\hline & no hypotension & 1.6 & \pm 1.3 & 2.8 & \pm 1.9 & & & \\
\hline
\end{tabular}

Data are presented as mean \pm standand deviation. MAP, mean arterial pressure (mmHig); CVP, central venous presssure

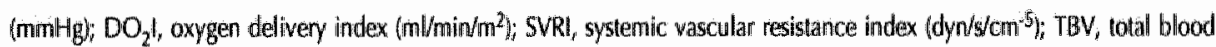
volume; RHEDV, right-heart end-diastolic volume; RVEDV, right ventricle end-diastolic volume; RVSW, right ventricle stroke wark (g/m/m² ); LVSW, lefi-wentricle stroke work (g/m/m² $\left(\mathrm{kPa} ; \mathrm{Pr}_{\mathrm{ret}} \mathrm{CO}_{2}\right.$, difference between regional and end-tidal $\left.\mathrm{PCO}_{2} ; \mathrm{CB}\right)$, indocyanine green blood clearance $\left(\mathrm{ml} / \mathrm{min} / \mathrm{m}^{2}\right) ; \mathrm{CPI}$, indocyanine plasma clearance $\left(m / / m i n d m^{2}\right)_{;}$; POR indocyanine plasma disappearance rate (\%). Comparison using multiple-way ANOVA; $\mathbb{P}_{\mathrm{T}}=p$-value for time-effect, $\mathbb{P}_{\mathrm{G}_{\mathrm{F}}}=p$-value for group effect, $\mathrm{P}_{\mathrm{T}_{\mathrm{C}}}=\mathrm{p}$-value for time-group interaction.

\section{Admission variables}

At admission to the ICU before resuscitation was initiated patients with hypotension had, by definition a significantly lower MAP (Table 2) ( $p<0.0001$ ). A lower LVSWI was also present at admission in hypotensive patients (Table 2) $(p=0.007)$. Moreover, in these patients a lower $\mathrm{CBI}(\mathrm{p}=0.009)$ and $\mathrm{CPI}$ 
$(p=0.02)$ were found (Table 2). The gastric pHi was significantly lower in the patients with hypotension $(p=0.04$ ). Finally, urine production was significantly lower in patients admitted with shock $(p=0.04)$. Apparently, both parameters of global perfusion and regional function were different between the two patient groups. None of the global or regional parameters at admission were more important in predicting mortality than another parameter on ROC-curve analysis (Table 3), although gastric $\mathrm{pHi}$ and $\mathrm{CP}$ t tended to be more important predictors of mortality on logistic regression analysis: $(p=0.06$ and $p=0.09$ for $\mathrm{pHi}$ and $\mathrm{CPI}$, respectively).

Table 3 Area under the receiver operator characteristic curves

\begin{tabular}{|c|c|c|c|c|c|}
\hline \multirow{2}{*}{$\begin{array}{l}\text { Parameter } \\
\mathrm{CBI}\end{array}$} & \multicolumn{2}{|c|}{$\begin{array}{l}\text { Admission } \\
\text { AUC }\end{array}$} & \multicolumn{2}{|c|}{$\begin{array}{l}\text { After stabilisation } \\
\qquad \text { AUC }\end{array}$} & Odds ratio ( $95 \% \mathrm{Cl})$ \\
\hline & 0.69 & \pm 0.08 & 0.70 & \pm 0.08 & $3.9(1.1-13.8)$ \\
\hline MAP & 0.55 & \pm 0.05 & 0.57 & $\pm 0.06^{b c}$ & $0.8(0.3-1.8)$ \\
\hline $\mathrm{pH}$ & 0.65 & \pm 0.05 & 0.75 & $\pm 0.05^{a}$ & $4.8(1.5-14.6)$ \\
\hline $\mathrm{PrCO}_{2}$ & 0.56 & \pm 0.05 & 0.65 & $\pm 0.06^{b}$ & $2.0(0.9-4.5)$ \\
\hline Pretco & 0.61 & \pm 0.06 & 0.75 & $\pm 0.07^{a}$ & $3.0(1.4-6.3)$ \\
\hline $\mathrm{P}_{\mathrm{N}-\mathrm{aCO}}$ & 0.59 & \pm 0.06 & 0.67 & \pm 0.09 & $1.3(0.6-3)$. \\
\hline CVP & 0.57 & \pm 0.06 & 0.56 & $\pm 0.08^{b c d}$ & $0.8 \quad(0.2-2.9)$ \\
\hline LVSWI & 0.62 & \pm 0.06 & 0.60 & $\pm 0.08^{b c}$ & $1.8(0.6-5.6)$ \\
\hline Arteriall $\mathrm{pH}$ & 0.63 & \pm 0.06 & 0.63 & $\pm 0.08^{b c}$ & $2.1(0.7-6.2)$ \\
\hline
\end{tabular}

Area under the receiver operator characteristc curve (AUC) \pm standand error. For legends see table 2-3. Data indicate the differences in AUC at admission and after stabilization. The maximal value for the AUC is 1 . No differences in AUC were present at adrnission. The AUC of pHi and $\mathrm{PP}$-etCO 2 were significantly larger after stabilisation compared to admission values, a $p<0.05$ for time-point after stabilisation versus time-point at admission;

b $\mathrm{p}<0.05$ for comparison of $\mathrm{pH}$ il or $\mathrm{PretCO}_{2}$ verus marked variables; $\mathrm{p}_{\mathrm{p}}<0.05$ for $\mathrm{Pr}_{\mathrm{r} \text { etCO }}$ versus marked variables: s $<0.05$ for $\mathrm{CBI}$ versus marked varialibles. The odds ratios represent the increased risk of death for an optimal cut-off point of the test defined by analysis of the ROC-curves.

\section{Changes during resuscitation}

Prior to the protocolised resuscitation (prior to admission to the ICU) patients received a mean of 4.4 I of saline, and 3.3 I of Gelofusine, with no significant difference in volume infused between the hypotension and no hypotension group. This amount of fluids was given during the admission days prior to ICU admission (median 2.0 (range 0-95) days).

During this resuscitation phase MAP, CVP, $D_{2}$, and RVEF were increased significantly when all patients were taken together $(p<0.0001, p=0.04, p=0.04$, 


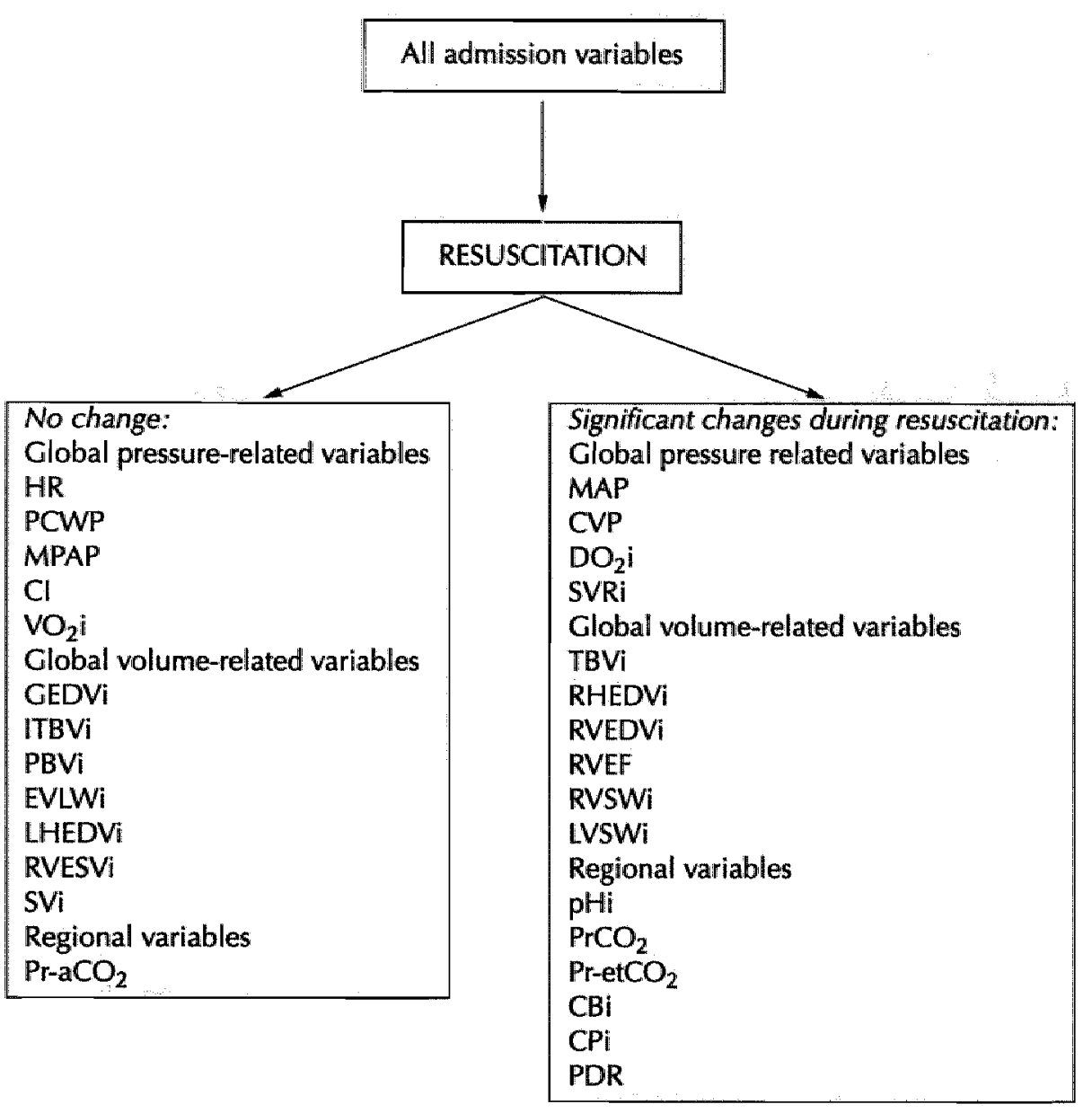

Schematic depiction of effects of ICU resusciation in patients with severe sepsis or septic shock on global pressurea and volume-related haemodynamic and regional parameters. A division is made between parameters found to be sigmificanily changed during resuscitation or between patients with and without shock (right panel) and parameters found to be similar during the study period (left panell).

Figure 2

and 0.03 for MAP, CVP, DO, and RVEF, respectively). Resuscitation only tended to increase LVSWI $\mathrm{Cl}$, and urine ouput ( $p=0.06,0.08$, and 0.08 , respectively), all other variables remained similar (Figure 2 and Table 2).

Significant differences existed during this whole time-period between patients with and without hypotension with respect to MAP, CVP, SVRI, $\mathrm{PrCO}_{2}, \mathrm{Pr}$-etCO, $\mathrm{pHi}$, lactate, base deficit, PaCO ${ }_{2}$, RHEDVI, RVEDVI, RVSWI, LVSWI, TBVI, CBI- and CPI-dye, and PDR-dye (Table 2). 
However, during the resuscitation efforts only the MAP and CVP were increased significantly more in patients with hypotension than in patients without hypotension (both $p=0.03$ ). All other parameters did not change significantly comparing the response in patients with and without hypotension (Table 2).

\section{Haemodynamic parameters after stabilisation as predictors of mortality}

After stabilisation was achieved the global haemodynamic and regional parameters were compared between surviving and non-surviving patients. After stabilisation a significantly higher $\mathrm{PrCO}_{2}, \mathrm{Pr}$-etCO $\mathrm{CO}_{2}$ and a significantly lower $\mathrm{CBI}, \mathrm{CPI}, \mathrm{PDR}$, and pHi were present in patients who died during their hospital stay (Table 4).

Table 4 Global haemodynamic and regilonal parameters after stabilisation in surviving and non-surviving patients

\begin{tabular}{lccccc}
\hline Parameters & \multicolumn{2}{c}{ Surviving } & Non-surviving & p-value \\
\hline $\mathrm{pHi}$ & 7.31 & \pm 0.07 & 7.24 & \pm 0.09 & 0.03 \\
$\mathrm{Pr}_{\mathrm{retCO}}$ & 2.1 & \pm 0.7 & 3.1 & \pm 0.5 & 0.04 \\
$\mathrm{PrCO}_{2}$ & 6.0 & \pm 0.8 & 7.1 & \pm 1.5 & 0.04 \\
$\mathrm{CB}$ & 838 & \pm 295 & 572 & \pm 26. & 0.02 \\
$\mathrm{CPI}$ & 587 & \pm 224 & 411 & \pm 168 & 0.02 \\
$\mathrm{PDR}$ & 21.1 & \pm 7.1 & 14.6 & \pm 5.0 & 0.01 \\
\hline
\end{tabular}

Data are presented as meam \pm standard deviation. For legends see table 2-3. Only parameters which showed a significant difference were displayed, Compaison between parameters after stabilisation was achieved between survivors and non-sunwivors.

The variables, which remained significantly different between surviving and non-surviving patients after the stabilisation period, were subsequently used as independent variables in a logistic regression analysis with mortality as dependent parameter and areas under the ROC-curves were calculated. The most important predictors were $\mathrm{CBI}$, gastric intramucosal $\mathrm{pHi}$, mucosal-end-tidal $\mathrm{PCO}_{2}$ gap, and the $\mathrm{PrCO}_{2}(\mathrm{CB}): \mathrm{B}=-0.002, \mathrm{SE}=0.0007, \mathrm{p}=0.01 ; \mathrm{pHi}: \mathrm{B}=-10.9$, $\mathrm{SE}=4.6, \mathrm{p}=0.02 ; \mathrm{PrCO}_{2}: \mathrm{B}=1.5, \mathrm{SE}=0.7, \mathrm{p}=0.03 ; \mathrm{Pr}_{\mathrm{etCO}}: \mathrm{B}=1.5, \mathrm{SE}=0.7$, $\mathrm{p}=0.02$; constant: $\mathrm{B}=83.9, \mathrm{SE}=35.1$ ). The AUCs of the gastric $\mathrm{pHi}$ and $\mathrm{Pr}$-etCO were significantly higher after stabilisation than at admission and were significantly higher than the AUC of MAP, $\mathrm{PrCO}_{2}, \mathrm{CVP}$, LVSWI, and arterial pH (Table 3). 


\section{Discussion}

The main findings of this study were: 1) No superior predictor of outcome could be identified at admission, although both regional parameters and global haemodynamic parameters were different between patients with and without shock and in both groups the mean values were abnormal compared to standard normal values. 2) After stabilisation regional parameters were the most important predictors of mortality. 3) Resuscitation aimed at improving global haemodynamics did improve global pressure-related and oxygen transport parameters, but not volume-related global haemodynamic and regional parameters. Previously, only a few studies have compared the predictive power of gastric tonometric variables with systemic haemodynamic variables. Bams et al. determined the predictive value of gastric pHi in cardiac surgical patients (23). In contrast to our study they found the mean arterial pressure and mean pulmonary arterial pressure more predictive of mortality than gastric pHi or $\mathrm{PrCO}_{2}$ when measured at admission and after 12 $h$. The difference between our study and this study may be related to the patient population selected. The presence of extra-splanchnic perfusion abnormalities, such as decreased bicarbonate content, may be another explanation for the differences. However, this cannot explain the predictive power of the $\mathrm{PrCO}_{2}$ and the $\mathrm{Pr}$-etCO in our study. Perhaps more important is the fact that in our study gastric tonometric variables became predictive after stabilisation was achieved, usually more than 12 $\mathrm{h}$ after admission. Maynard et al. confirmed this result by finding a high predictive value for the gastric pHi only after 3 days (24). On the other hand, Lorente et al. (25) found that the $\mathrm{Pr}-\mathrm{aCO}$ gap was an independent predictor of outcome in severe burn patrients only at admission and not after $12 \mathrm{~h}$ of resuscitation. Gastric $\mathrm{PrCO}_{2}$ and systemic lactate were both good predictors of outcome in patients with severe sepsis in another study (26). Accordingly, Ivatury et al. indicated that gastric $\mathrm{pHi}$ was the best predictor of outcome in severely traumatised patients with organ failure (27). Thus, several studies confirmed the importance of gastric intramucosal tonometry in predicting outcome in critically ill patients.

The importance of volume-related global haemodynamic parameters in the assessment of intravascular volume status has been emphasized before. Numerous studies have indicated that intravascular volumes assessed by thermo-dye dilution technique give more accurate information concerning the preload dependency of cardiac output than pressure-related parameters (15,28-32). In addition, our study investigated the predictive power of the global volume-related parameters in patients with septic shock. We found that volume-related haemodynamic parameters were superior to pressure-related parameters in differentiating between survivors and non-survivors. Previously, Jaarsma et al. found that the predictive power of LVSWI using echocardiography was higher than that of PCWP in patients with acute myocardial infarction (33). In our study resuscitation increased RVEF both in patients with and without hypotension, indicating that in all patients resuscitation was incomplete. It is interesting to see that the extravascular lung water tended to decrease during resuscitation. This decrease in EVLW may indicate improved fluid balance or may be due to the increased application of PEEP ventilation (34). 
Intensive care resuscitation is ailmed at improwing the global perfustion. Shoemaker et al. described that surviving patients achieved higher oxygen transport and perfusion variables during their stay on the intensive care unit (2). In addition Moore et al. found that in critically ill trauma patients the inability to improve oxygen transport during resuscitation aimed at achieving supranormal oxygen delivery was related to the development of multiple organ failure (1). However, it remained difficult to identify the individual patient who was prone to develop multiple organ failure or die on the intensive care. Rhodes et al. described a dobutamine-stress test, which can identify non-survivors (35). Creteur et al. found that this stress test identifies occult hypovolaemia in the splanchnic area (36). In addition, in our study, patients with shock at admission had obvious abnormalities in both global, gastric tonometric and ICG-clearance variables, whereas after stabilisation abnormalities in the hepatosplanchnic parameters still existed.

There are a number of remarks, which must be made when interpreting these observations. The resuscitation principles used in this study were based on standard practice on our $\mathrm{ICU}$ and the intensivist on duty judged whether the resuscitation led to stabilisation of the global haemodynamics. Although this practise led to improvement of global haemodynamic parameters no improvement in, and even a worsening of, the reglional parameters could be noted. It may be questioned whether the resuscitation used was adequate and whether other methods of resuscitation should have been attempted. However, our aim was to study the effects of normally used resuscitation techniques in an intensive care unit. The tendency of the global volume-related parameters stroke volume and RVEF to improve and the significant improvement in oxygen delivery during this resuscitation indicate that the resuscitation led to improvement of the global haemodynamics. The question of which resuscitation techniques should be applied in patients with septic shock remains difficult to answer. The use of 'supranormal' haemodynamic goals in patients with septic shock and organ failure has been shown to have no influence on outcome $(37,38)$ and even increased mortality in some studies (39). On the other hand, in a recent trial the application of these supranormal goals early in the course of septic shock resulted in a reduced mortality (40).

Moreover, the number of patients in this prospective study is relatively small and this may introduce, together with the multiple comparisons, type II erorrs. This study should be regarded as an initial study for the design of a larger trial comparing the different monitoring modalitites. However, even with the relatively small numbers studied, we found important differences in the predictive capability between global haemodynamic and regional parameters.

In conclusion, this study showed that during standard intensive care resuscitation in patients with septic shock, parameters of splanchnic function are better predictors of outcome than global pressure- or volume-related parameters. Before resuscitation is started no superior predictor of outcome could be identified. 


\section{REFERENCES}

1. Moore FA, Haenel JB, Moore $\mathrm{EE}_{2}$, Whitehill TA. Incommensurate oxygen consumption in response to maximal oxygen availability predicts postinjury multiple organ failure. I Trauma $1992 ; 33(1): 58-65$.

2. Bishop $M H$, Shoemaker $W C_{r}$ et al. Relationship between supranormal circulatory values, time delays, and outcome in severely traumatized patients. Crit Care Med 1993,21(1):56-63.

3. Dabrowski GP, Steinberg SM, Ferrara II, Flint LM. A critical assessment of endpoints of shock resuscitation. Surg Clin North Am 2002;80(3):825-44.

4. Wo CC, Shoemaker WC, Appel PL, Bishop MH, Kram HB, Hardin E. Unreliability of blood pressure and heart rate for evaluation of circulatory stability in emergency resuscitation and critical illness. Crit Care Med 1993;21:218-23.

5. Brinkmann $A$, Calzia $E$, Träger $K$, Radermacher $P$. Monitoring the hepato-splanchnic regiona in the critically ill patient. Measurement techniques and clinical relevance. Intensive Care Med 1998;24:542-56.

6. Santoso IT, Wisner DH, Battistella FD, Owings IT. Comparison of gastric mucosal pH and clinical judgement in critically ill patients. Eur / Surg 1998;164:521-6.

7. Oud $L$, Kruse JA. Progressive gastric intramucosal acidosis follows resuscitation from hemorrhagic shock. Shock 1996;6(1):61-5.

8. Poeze M, Takala J, Greve JWM, Ramsay G. Pre-operative tonometry is predictive for mortality and morbidity in high-risk surgical patients. Intensive Care Med $2000 ; 26(9): 1272-81$.

9. Edouard AR, Dergremont A-C, Duranteau ], Pussard E, Berdeaux A, Samï K. Heterogeneous regional vascular responses to simulated transient hypovolemia in man. Intensive Care Med 1994;20:414-20.

10. Kirton $\mathrm{O}$, Windsor $\mathrm{J}$, Wedderburn $\mathrm{R}$, et al. Failure of splanchnic resuscitation in the acutely injured trauma patient correlates with multiple organ system failure and length of stay in the ICU. Chest 1998;113(4):1064-169.

11. Poeze M, Ramsay G, Buurman WA, Greve JWM, Dentener M, Takala I. Increased hepatosplanchnic inflammation precedes the development of organ dysfunction after elective high-risk surgery. Shock 2002;17(6):451-458.

12. Singer $M$, Bennett D. Pitfalls of pulmonary artery catheterization highlighted by Doppler ultrasound. Crit Care Med 1989;17(10):1060-1.

13. Godje $O$, Peyerl $M_{r}$ Seebauer T, Lamm $P$, Mair $H$, Reichart B. Central venous pressure, pulmonary capillary wedge pressure and intrathoracic blood volumes as preload indicators in cardiac surgery patients. Eur \& Cardiothorac Surg 1998;13(5):533-9.

14. Chang MC, Meredith IW. Cardiac preload, splanchnic perfusion, and their relationship during resuscitation in trauma patients. I Trauma 1997;42(4):577-82.

15. Lichtwarck-Aschoff $M$, Beale R, Pfeiffer U. Central wenous pressure, pullmonary artery occlusion pressure, intrathoracic blood volume, and right ventricular end-diastolic volume as indicators of cardiac preload. I Crit Care 1996:11(4):180-8.

16. Holm C, Melcer B, Horbrand F, Worl H, von-Donnersmarck GH, Muhlbauer W. Intrathoracic blood volume as an end point in resuscitation of the severely burned: an observational study of 24 patients. J Trauma 2000;48(4):728-34.

17. Bone $R C$, Balk RA, Cerra $F B$, et al. Definitions for sepsis and organ failure and guidelines for the use of innovative therapies in sepsis. Chest 1992;101(6):1644-1655. 
18. Gutierrez $\mathrm{G}$, Palizas $\mathrm{F}$, Doglio $\mathrm{G}$, et al. Castric intramucosal $\mathrm{pH}$ as a therapeutic index of tissue oxygenation in critically ill. Lancet 1992;339(1):195-9.

19. Reilly PM, Bulkley CB. Vasoactive mediators and splanchnic perfusion. Crit Care Med $1993 ; 210$ Suppl):\$55-\$68

20. Poeze $M$, Greve JWM, Ramsay $G$. Is splanchnic perfusion a critical problem in sepsis? In: Baue $A E_{i}$ Berlot $C_{i}$ Gullo $A$, Vincent $J-L$ (eds). Siepsis and organ dysfunction. From basics to clinical approach. First edition. Milano, Springer-Verlag, 1999, pp.169-81.

21. Hanley JA, MclNeil Bj. A method for comparing the areas under receiver operating characteristic curves derived from the same cases. Radiology 1983;148:839-43.

22. Hanley $I A, M c N e i l l B$. The meaning and use of the area under a receiver operating characteristic (ROC) cunve. Radiology 1982;143:29-36.

23. Bams JL, Mariani MA, Groeneveld AB. Predicting outcome after cardiac surgery: comparison of global haemodynamic and tonometric variables. Br J Anaesth 2000;82(1):33-7.

24. Maynard ND, Bihari $D$, Beale $R$, et all. Assessment of splanchnic oxygenation by gastric tonometry in patients with acute circulatory failure. JAMA 1993;270(10):1203-10.

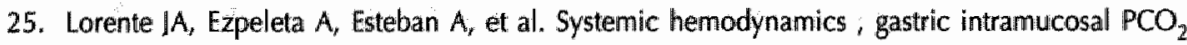
changes, and outcome in critically ill burn patients. Crit Care Med 2000;28(6):1728-1735.

26. Friedman $G$, Berlot $G$, Kahn $R$, Vincent $J-L$. Combined measurements of blood lactate concentrations and gastric intramucosal $\mathrm{pH}$ in patients with severe sepsis. Crit Care Med $1995 ; 23(7): 1184-93$.

27. Ivatury RR, Simon RJ, Havriliak D, Garcia C, Greenberg J, Stahl WM. Gastric mucosal pH and oxygen delivery and oxygen consumption as indices in the assessment of adequacy of resuscitation after trauma: a prospective randomised study. I Trauma 1995;39(1):128-136.

28. Brock $H$, Gabriel $C$, Bibl D, Necek S. Monitoring intravascular volumes for postoperative volume therapy. Eur \& Anaesthesiol 2002;19(4):288-94.

29. Bindels $A J$, van der Hoeven JG, Graafland AD, Koning J, Meinders AE. Relationships between volume and pressure measurements and stroke volume in critically ill patients. Crit Care 2000;4(3): $193-9$.

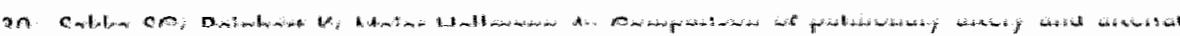
thermodilution cardiac output in critically ill patients. Intensive Care Med 1999;25(8):843-6.

31. Kisch $H$, Leucht $S$ Lichtwarck-Aschoff $M$, Pfeiffer UJ. Accuracy and reproducibility of the measurement of actively circulating blood volume with an integrated fiberoptic monitoring system. Crit Care Med 1995;23(5):885-93.

32. Lichtwarck-Aschoff $M$, Zeravik J, Pfeiffer UJ. Intrathoracic blood volume accurately reflects circulatory volume status in critically ill patients with mechanical ventilation. Intensive Care Med 1992;18:142-7.

33. Jaarsma W, Visser CA, Eenige van MI, Verheugt FW, Kupper $A$, Roos JP. Predictive value of two-dimensional echocardiographic and hemodynamic measurements on admission with acute myocardial infarction. I Am Soc Echocardiogr 1998;1(3):187-93.

34. Mitchell JP, Schuller D, Calandrino FS, Schuster DP. Improved outcome based on fluid management in critically ill patients requiring pulmonary artery catheterisation. Am J Resp Crit Care Med 1992:145:990 998.

35. Rhodes A, Malagon I, Lamb Fl, et al. Failure to increase oxygen consumption is a predictor of mortality in septic patients. Intensive Care Med 1996;\$274 
36. Creteur J. De-Backer D, Vincent $1-4$ A dobutamine test can disclose hepatosplanchnic hypoperfusion in septic patients. Am J Respir:Crit Care Med 1999,160(3):839-45.

37. Poeze $M$, Greve JWM, Ramsay G. Oxygen delivery in septic shock. Chest 1999;116(4): 1145 [letter]

38. Boyd O, Hayes M. The oxygen trail: the goal, Br Med Bull 1999,55(1)125-39.

39. Hayes MA, Timmins AC, Yau EHS, Pallazzo M, Hinds C, Watson D, Elevation of systemic oxygen delivery in the treatment of critically ill patients. New Eng I Med 1994;330(24):1717-22.

40. Rivers E, Nguyen B, Havstad S, et al. and Early Goal-Directed Therapy Collaborative Group: Early goal-directed therapy in the treatment of severe sepsis and septic shock New Eng J Med $2001 ; 345(19): 1368-77$. 
CHAPTER 8

D-lactate as an early marker

of intestinal ischaemin after

ruptured abdominal aneurysm

Published in

Br J Surg, 1998; 85(9):1221-1224. 


\section{ABSTRACT}

Objective: Patients with acutely ruptured abdominal aortic aneurysm (ruptured AAA) are at risk of developing colonic ischaemia after surgery. It is difficult to diagnose this ischaemia at an early stage. D-lactate is produced by intestinal bacteria after ischaemia. L-lactate is released in increased amounts during hypoxia by anaerobic metabolism. This study investigated both variables as a marker for intestinal ischaemia in patients with a ruptured AAA.

136 Design Retrospective, observational study.

Setting: Intensive care unit in an university-affiliated hospital.

Patients: Patients operated upon with ruptured AAA, elective AAA, and patients with pneumonia admitted to the intensive care unit and healthy controls.

Interventions: No planned interventions.

Measurements: Twenty-four patients with ruptured AAA were divided retrospectively into two groups with and without ischaemic complications, as verified by colonoscopy. Blood had been taken on admission to the intensive care unit. Median time to colonoscopy was 9 days after surgery. As controls, four patients with pneumonia, six healthy subjects, five patients with an elective AAA, and six patients with sepsis and acute tubular necrosis were included.

Main results. D-lactate level on admission was significantly increased in patients with colonic ischaemia after ruptured AAA compared with the level in patients without ischaemia $(p<0.05)$, patients with sepsis $(p<0.001)$, those with pneumonia and healthy subjects $(p<0.01)$. L-lactate concentration was similar in the group with intestinal complications and in patients without colonic ischaemia; however, L-lactate levels were higher in patients with pneumonia and sepsis than in healthy subjects $(p<0.05)$.

Conclusions: On admission to the ICU, D-lactate, but not L-lactate levels, may predict later colonic ischaemia following repair of a ruptured AAA. 


\section{INTRODUCTION}

After repair of a ruptured abdominal aortic aneurysm (AAA), patients are at risk of developing colonic ischaemic, due to the preceding hypoperfusion of the intestine as a result of the systemic shock and aortic clamping. Furthermore, loss of flow to the colon via the inferior mesenteric artery, and/or loss of flow via one of both internal iliac arteries may contribute. The overall complication rate following repair of a ruptured AAA averages about $50 \%(1)$. Gastro-intestinal and systemic problems encountered range from diarrhoea $(43 \%)(2)$, to ischaemic colitis $(27 \%)$ $(3,4)$, bowel necrosis $(2-35 \%)(5)$ or overt sepsis with organ failure $(20 \%)(6)$. The mortality rate associated with these complications may be as high as $75 \%$ (7) up to $90 \%$ in patients with transmural involvement of the colonic wall (8). Early surgical intervention in the acute phase and improved pre- and postoperative care reduces this complication rate (9).

The diagnosis of colonic ischaemia after aneurysm repair is difficult, because of the non-specific clinical signs in the early phase. In most patients, colonic ischaemia is recognised only when the patient has extensive colonic necrosis or sepsis (10).

Colonoscopy is the best method for diagnosing colonic ischaemia, but this technique is invasive and is usually performed only in patients with overt signs of bowel ischaemia (11). The measurement of gut intramucosal $\mathrm{pH}$ may be useful, but the cost of monitoring all patients with a tonometry-catheter is relatively high $(2,12)$. A serum marker of colonic ischaemia may be useful, but at present no marker has been found that is sensitive and specific enough. Lactate has been studied in the diagnosis of acute abdominal disease (13). L-lactate is produced by all cells as a product of glycolysis in a balanced equilibrium during adequate oxygen supply (14). During hypoxia or flow arrest, lactate is produced in excess and is released into the bloodstream. However, the lactate produced by the gut is largely extracted by the liver (15). Raised L-lactate levels are, therefore, related to inadequate tissue perfusion, but the measurement may be limited by several confounding factors.

The stereo-isomer D-lactate is produced only by bacteria as a normal product of bacterial fermentation (16). The exact mechanism by which D-lactate enters the bloodstream is unknown, but baseline levels in healthy subjects are very low. Under certain conditions, however, the number of bacteria can increase rapidly correlating with an increased level of D-lactate. This has been described in patients with bacterial overgrowth due to infection (16), short bowel syndrome (17), and to mesenteric infarction causing colonic ischaemia (18).

It is not known whether acute surgical intervention for ruptured abdominal aneurysm causes a rise in serum D-lactate, and whether plasma levels are diagnostic for colonic ischaemia.

In this study patients with colonic ischaemia after repair of a ruptured AAA were compared with patients who had the same surgical procedure without this complication. It was hypothesized that the measurement of D-lactate concentration immediately after operation on arrival in the intensive care unit (ICU) may be a better marker for colonic ischaemic complications than L-lactate. 


\section{PATIENTS AND METHODS}

The patients were selected from prospectively acquired database of all those admitted to the ICU from January 1990 to March 1994. Eighty-two patients were admitted to the ICU after surgery for AAA. Of these, 24 patients with ruptured AAA were selected. The patients were included if sufficient clinical data were available and if plasma samples had been taken on ICU admission. Several patient groups were selected as controls. First, six healthy subjects were used for base-line measurement of D-lactate. As a control group for critically ill patients without colonic ischaemia, six patients admitted for pneumonia or who developed a ventilatory associated pneumonia, in need of ventilatory support were used. A further group consisted of five patients undergoing elective AAA repair. The majority of patients following elective AAA are not admitted to the ICU in this institution; these selected patients were therefore at increased risk of developing complications. Finally, a group of six patients with abdominal septic shock and acute tubular necrosis who did not undergo aneurysm surgery was selected, because of the frequent relation between this condition and colonic ischaemia after a ruptured AAA (6). All patients with aneurysms were compared haemodynamically, in relation to the presence of presurgical shock (mean arterial pressure (MAP) $<70 \mathrm{mmHg}$ ), the duration of aortic clamping, the lowest MAP during surgery and the estimated blood loss during the procedure.

Patients with ischaemic colitis, confirmed by colonoscopy, were allocated retrospectively in the ischaemic group. Colonic ischaemic changes were divided into three categories, as described by Hagihara et al. (19).

D-lactate concentration was determined from serum collected after operation on admission to the $\| \mathrm{CU}$. The sample was mixed with ethylenediamine tetraacetic acid (EDTA) centrifuged at $4{ }^{\circ} \mathrm{C}$ at $1500 \mathrm{rpm}$ for 20 minutes, and $4 \mathrm{ml}$ of plasma was stored at $-70{ }^{\circ} \mathrm{C}$ until measurement. After thawing plasma was deproteinized with perchloric acid $(1 / 1$ vol), mixed thoroughly, and kept on ice for 30 minutes after which the supernatant solution was removed. This was mixed with a nicotamide adenine inucleotide (NAD) ${ }^{+}$-glycine-hydrazine solution to form pyruvatehydrazon with reduced $\mathrm{NAD}(\mathrm{NADH})$ and $\mathrm{H}^{+}$, at a $\mathrm{pH}$ of less then 9.0. This reaction was initiated by adding D-lactate dehydrogenase (D-LDH). The perchloric acid was not mixed at the time of collection, as described before, but at time of measurement after storage (20). Both techniques yielded similar D-lactate levels (standard error of differences 0.01 ) (20).

For quantification of L-lactate in serum, the same technique was used as described above, but with replacement of the D-lactate standard and D-LDH by L-lactate and $\mathrm{L}$-LDH. D-lactate levels of $<0.1 \mathrm{mmol} / \mathrm{l}$ and L-lactate levels of $<2.0 \mathrm{mmol} / \mathrm{l}$ were considered to be normal.

Data are presented as mean (SD) or percentages. Student's t-test or chi-square test were used to determine statistical differences between groups. Sensitivity and specificity were calculated according to standard methods. A p-value of less than 0.05 was considered statistically significant. 


\section{RESULTS}

\section{Patient demographics}

In total, 24 patients with ruptured AAA were assessed. Ischaemic collitis was diagnosed in 11 of these patients. A large proportion (7/11) of the patients with acute aneurysm repair followed by intestinal complications had significant complications from dysfunction of other organ systems, for example acute tubular necrosis or sepsis. Of these patients, six (55\%) died, one from rebleeding and five from late organ failure with sepsis. The demographic details of the control groups with pneumonia, elective aneurysm repair and sepsis were similar.

Aortic clamping time was longer in the group with a ruptured AAA with ischaemic complications than in the patients with an elective AAA and without ischaemic complications $(p<0.05)$. Also, the three aneurysm subgroups differed with regard to peri-operative haemodynamic state (table 1).

Table 1 Comparison of patient demographics between patients with acute or ellective aneurysm repair

\begin{tabular}{|c|c|c|c|c|c|c|}
\hline 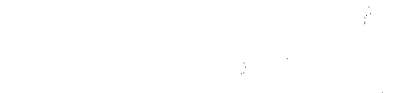 & \multicolumn{2}{|c|}{ ruptured AAA } & \multicolumn{2}{|c|}{$\begin{array}{l}\text { ruptured AAA } \\
+ \text { ischaemia }\end{array}$} & \multicolumn{2}{|c|}{ elective AAA } \\
\hline Number & \multicolumn{2}{|c|}{13} & \multicolumn{2}{|c|}{11} & \multicolumn{2}{|c|}{5} \\
\hline Age (years) & 71.2 & $(8.5)$ & 71.1 & $(11.7)$ & 61.6 & $(13.4)$ \\
\hline $\operatorname{Sex}(M / F)$ & \multicolumn{2}{|c|}{$11 / 2$} & \multicolumn{2}{|c|}{$11 / 0$} & \multicolumn{2}{|c|}{$5 / 0$} \\
\hline Pre-surgical shock $(\%)$ & $5 / 10$ & $(50)$ & $8 / 11$ & (73) & $0 / 4$ & $(0)^{\mathrm{a}}$ \\
\hline Lowest MAP during surgery (mimHg) & 67.4 & $(42.0)$ & 61.1 & $(13.0)$ & 74.5 & $(19.2)$ \\
\hline Duration of shock ( $\mathrm{min}$ ) & 71 & $(58)$ & 82 & (45) & 26 & $(10)^{A}$ \\
\hline Blood loss (l) & 7.4 & $(6.5)^{\mathrm{a}}$ & 5.3 & (6.4) & 1.4 & $(0.9)$ \\
\hline Clamping time & $68: 9$ & $(43.8)$ & 108.9 & $(52.2)^{\mathrm{a}}$ & 55.4 & $(33.7)$ \\
\hline Survival $(\%)$ & $7 / 13$ & (54) & $5 / 11$ & (45) & $5 / 51$ & 100 \\
\hline
\end{tabular}

All groups were comparable with respect to age, sex, the lowest mean arterial pressure (MAP) during surgery, and survivall. Figures are presented as mean (standard deviation) or as absoluie number (percentages), $\mathrm{p}<0.05$ for pre-surgical shock; elective AAA vs, ruptured AAA + ischaemia and ruptured $A A A$, duration of shock during surgery; elective AAA vs, ruptured $A A A+$ ischaemia and ruptured AAA, blood loss; ruptured AAA ws. elective AAA, and clamping time; ruptured AAA + ischaemia ws. ruptured AAA and elective AAA. 


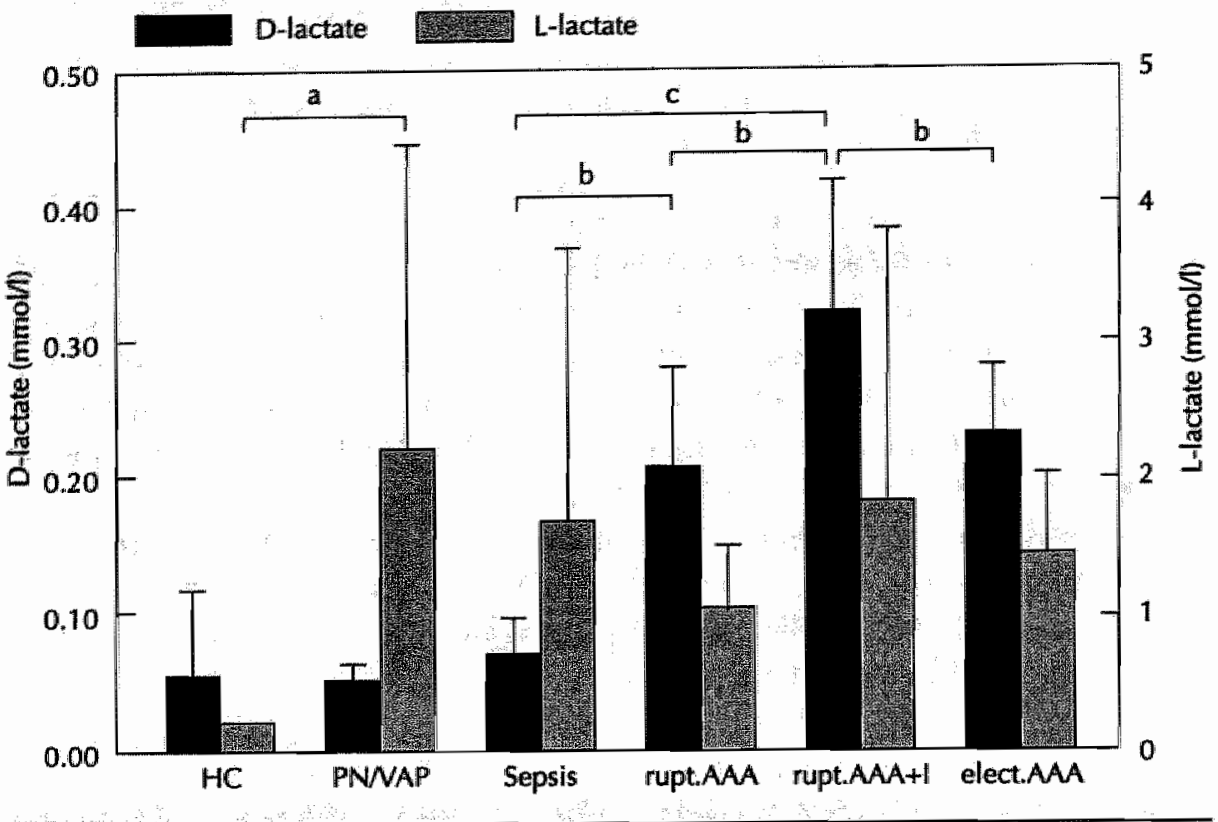

D. Lactate and $\mathrm{L}$-lactale levels for the different groups. Vallues are mean $(\mathrm{SD}), \mathrm{HC}=$ healthy controls: PNWAP = pneumomia/ ventilatory associated pneumonia; Sepsis = septic shock and acute fubular necrosis; rupt. $A A A=$ npptured aneurysm of abdominal aorta" $A A A=$ elective aneurysm of abdominal aota; $+1=$ with colonic ischalemia; ${ }^{a} p<0.05, b p<0.01, " c p<0.001$.

Figure 1

\section{D-lactate and L-lactate levels}

The mean time from aortic clamping until blood sampling was 6.3 (range 3-12) $\mathrm{h}$ in the ruptured AAA group. The median duration from the blood sampling time until colonoscopy was 9 (range 2-16) days. Mean (SD) D-lactate and L-lactate levels are shown in figure 1. D-lactate levels were significantly higher in patients after ruptured AAA with colonic ischaemic complications compared with levels in patients with no such complications $(p<0.01)$ and after elective AAA $(p<0.01)$. Mean D-lactate levels in healthy subjects, patients with pneumonia, and in those with septic shock with acute tubular necrosis were not raised above baseline. No differences in L-lactate level between patients with pneumonia or septic shock and those with and without colonic ischaemia could be found. L-lactate levels in healthy controls, however, were significantly lower than those in patients with pneumonia and with septic shock and acute tubular necrosis $(p<0.05)$.

If a cut-off point of the D-lactate concentration of greater than $0.20 \mathrm{mmol} / \mathrm{l}$ is taken in patients with ruptured AAA, then the sensitivity for prediction of ischaemic colitis was $82 \%(9 / 11)$ with a specificity of $77 \%(10 / 13)$, with a negative predictive 
value and positive predictive value of $75 \%$ and $83 \%$, respectively. One patient had a low D-lactate $(0.16 \mathrm{mmol} / \mathrm{l})$ on admission to the ICU, 3hrs after surgery. The next day the level of $D$-lactate was already 0.45 and remained high. On day 10 after surgery ischaemic colitis grade 3 was diagnosed on endoscopy.

\section{DisCussion}

This study was undertaken in order to investigate the value of measuring D-lactate or L-lactate concentration in serum after acute aneurysm repair in prediction of colonic ischaemia. D-lactate is produced by several bacteria commonly found in the gut, such as Escherichia coli. It is probably not metabolized by the liver. Serum D-lactate levels in the peripheral blood may closely represent the concentrations in the portal vein. In a previous study in rats, Murray et al., showed increased levels of D-lactate in peripheral blood samples $2 \mathrm{hrs}$ after ligation of the superior mesenteric artery (21). In this study D-lactate concentration was raised at a mean of $6 \mathrm{hrs}$ after the start of aortic clamping in patients who later (median 9 days) developed ischaemic colitis. The increased D-lactate levels may be due to an increased intestinal permeability $(22,23)$. One of the earliest manifestations of splanchnic ischaemia is an increase in intestinal permeability, during either the hypoperfusion phase or during the reperfusion phase of aneurysm repair (24). Bacterial translocation occurs in both experimental and clinical conditions of intestinal hypoperfusion $(22,23)$, and has been related to the development of postoperative complications and organ failure $(25,26)$.

Another explanation for the increased D-lactate levels may be bacterial overgrowth, which can occur for several reasons, such as short bowel syndrome $(27,28)$. It has been shown that following colonic ischaemia the number of $E$. coli can increase rapidly (29), resulting in increased plasma D-lactate levels (30).

Normal D-lactate levels in patients with septic shock and acute tubular necrosis indicates that a period of complete ischaemia combined with systemic haemodynamic deterioration may be necessary to induce D-lactate release from the intestinal bacteria in the early postoperative phase. This may be supported by the finding of increased D-lactate levels in patients with mesenteric occlusion in the study by Murray et al., while patients with other causes for acute abdominal pain had normal levels (18).

In this study the sensitivity and specificity of the levels of D-lactate in predicting ischaemic complications were 82 and $77 \%$, respectively. Murray et al. found a sensitivity and a specificity of 90 and $87 \%$, respectively, for the levels of D-lactate in patients with mesenteric occlusion (18). This difference may be due to the retrospective selection of the patients in the present study. In patients with colonic ischaemia after ruptured AAA, only those with the most severe colonic ischaemia were included, because colonoscopy was performed only after clinical suspicion. 
Therefore, $\mathrm{D}$-lactate levels in patients without colonic ischaemia may have been higher, because those with mild ischaemic changes were also included in this subgroup. Irrespective of this possible bias, D-lactate seems to be a good marker to identify severe colonic ischaemia at an early stage.

The value of L-lactate in the assessment of adequacy of tissue perfusion and oxygenation is limited by several confounding variables, such as liver function, acute changes in acid-base balance, and interorgan exchange of lactate (31). Despite this, measurement of blood lactate levels provides a marker of metabolism and tissue perfusion (15). L-lactate levels were increased in patients with ruptured AAA compared to those in healthy subjects, and tended to be higher in patients with intestinal complications after ruptured AAA repair. However, L-lactate concentration was also higher in patients with pneumonia or septic shock than in healthy subjects, probably indicating the presence of tissue hypoxia in organs other than those of the gastro-intestinal tract. L-lactate levels were higher in patients with pneumonia than after an elective aneurysm repair. Since L-lactate, when produced in the intestinal tract, can be metabolised rapidly by the liver, this first pass effect may have caused the above mentioned difference. L-lactate produced in other tissues enters the systemic circulation directly.

The results of this study indicate that D-lactate may be a more specific marker for colonic ischaemia after surgery for a ruptured aortic aneurysm than L-lactate, which has a raised concentration in other critical illnesses. A prospective study is therefore justified to investigate the full value of determining $D$-lactate levels in patients at risk of colonic ischaemia following repair of a ruptured abdominal aneurysm. 


\section{REFERENCES}

1. Perler BA. The management of patients with ruptured abdaminal aortic aneurysms, Adv Surg 1997;30:21-38.

2. Soong CV, Blair PHB, Halliday MI, et al. Bowel ischemia and organ impairment in elective abdominal aortic aneurysm repair. Br / Surg 1994;81:965-968.

3. Bast TJ, van der Biezen J, Scherpenisse J, Eikelboom BC. Ischemic disease of the colon and rectum after surgery for abdominal aortic aneurysm: a prospective study of the incidence and risk factors. Eur J Vasc Surg 1990;4:253-257.

4. Maupin GE, Rimar SD, Villalba M. Ischemic colitis following abdominal aortic reconstruction for ruptured aneurysm. A 10-year experience. Am Surg 1989;55:378-380.

5. Ruckert RF, Buchmann P. ischaemische Colon- und Rectumnekrosen nach Resektion der Aorta abdominalis. Chirurg 1982;53:556-562.

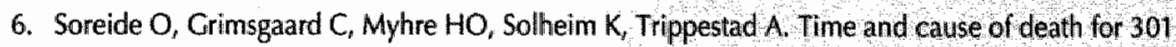
patients operated on for abdominal aortic aneurysms. Age Ageing 1982,11 256-260.

7. Zelenock $\mathrm{GB}_{\nu}$ Strodel $\mathrm{WE}, \mathrm{Knol} J \mathrm{~A}_{\nu}$ et al. A prospective study of clinically and endoscopically documented colonic ischemia in 100 patients undergoing aortic reconstructive surgery with aggressive colonic and direct pelvic revascularization, compared with historic controls, Surgery 1989;106:771-779.

8. Young JR, Humphries AW, deWolfe VG, Lefevre FA. Complications of aortic surgery. II. Intestinal ischemia. Arch Surg 1963;86:65-73.

9. Ernst CB. Prevention of intestinal ischemia following abdominal aortic reconstruction. Surgery 1983;93:102-106.

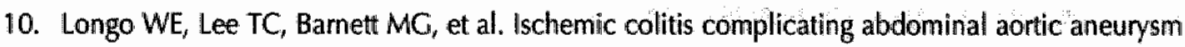
surgery in the US veteran. I Surg Res 1996;60:351-354,

11. Scherpenisse J, van Hees $P A$. The endoscopic spectrum of colonic mucosal injury following aortic aneurysm resection. Endoscopy 1989;21:174-176.

12. Nielsen VG, Tan S, Baird MS, MCCammon AT, Parks DA. Gastric intramucosal pH and multiple organ injury: impact of ischemia-reperfusion and xanthine oxidase. Crit Care Med 1996;24: 1339-1344.

13. Lange $H_{*}$ Jaeckel $R$. Usefulness of plasma lactate concentration in the diagnosis of acute abdominal disease. Eur I Surg 1994;160:381-384.

14. Brooks CA. Lactate production under fully aerobic conditions: The lactate shuttle during rest and exercise. Fed Proc 1986;45:2924-2929.

15. Takala J, Uusaro A, Parwiainen I, Ruokonen E. Lactate metabolism and regionall lactate exchange after cardiac surgery. New Horiz 1996;4:483-491.

16. Smith $S M$, Eng HIK, Buccini F. Use of D-lactic acid measurement in the diagnosis of bacterial infections. J Infect Dis 1986;154:658-664.

17. Oh MS, Phelps KR, Traube M, Barbosa-Saldivar JL, Boxhill C, Carroll HJ. D-lactic acidosis in a man with the short-bowel syndrome. New Eng J Med 1979;301:249-252.

18. Murray M), Gonze MD, Nowak LR, Cobb CF. Serum D(-)-lactate levels as an aid to diagnosing acute intestinal ischemia. Am I Surg 1994;167:575-578.

19. Hagihara PF, Ernst CB, Griffen WO. Incidence of ischemic colitis following abdominal aortic recontruction. Surg Gynecol Obstet 1979;149:571-573. 
20. Brandt $R B$, Siegel $S A$, Waters $M G$, Bloch $M H$. Spectrophotometric assay for $D-(-)$-lactate in plasma. Anal Biochem 1980;102:39-46.

21. Murray MJ Barbiose 11 , Cobb CF. Serum D(-H)hactate levels as a predictor of acute intestinal ischemia in a rat model. I Surg Res 1993;54:507-509.

22. Deitch EA, Morrison J Berg R, Specian RD. Effect of hemorrhagic shock on bacterial translocation, intestinal morphology, and intestinal permeability in conventional and antibioticdecontaminated rats. Crit Care Med 1990;18:529-536.

23. Sinclair $\mathrm{DG}_{2}$ Houldsworth PE, Keogh B, Pepper J, Evans TW. Gastrointestinal permeability following cardiopulmonary bypass: a randomised study comparing the effects of dopamine and dopexamine. Intensive Care Med 1997;23:310-316.

24. Ohri SK, Somasundaram S, Koak Y, et al. The effect of intestinal hypoperfusion on intestinal absorption and permeability during cardiopulmonary bypass. Gastroenterology 1994;106: 318-323.

25. Mythen $M G$, Webb $A R$. Perioperative plasma volume expansion reduces the incidence of gut mucosal hypoperfusion during cardiac surgery. Arch Surg 1995;130:423-429.

26. Lemaire LCM., van Lanschot JJB, Stoutenbeek $\mathrm{CP}$, van Deventer $\mathrm{S} \mathrm{H}$, Wells $\mathrm{CL}$; Gouma DJ. Bacterial translocation in multiple organ failure: cause or epiphenomenon still unproven. $\mathrm{Br}$ J Surg 1997;84:1340-1350.

27. Bongaerts $G$, Tolboom J, Naber $T$, Bakkeren J, Severijnen $R$, Willems H. D-lactic acidemia and aciduria in pediatric and adult patients with short bowel syndrome. Clin Chem 1995;41: 107-110.

28. Hove H, Mortensen PB. Colonic lactate metabolism and D-lactic acidosis. Dig Dis Sci 1995;40: 320-330.

29. Sykes PA, Boulter $\mathrm{KH}_{\text {, }}$ Schofield PF. The microflora of the obstructed bowel. Br I Surg 1976;63: 1966-1982.

30. Ouriel K, Fiore WM, Geary JE. Detection of occult colonic ischemia during aortic procedures: use of an intraoperative photoplethysmographic technique. I Vasc Sung 1988;7*5-9.

31. Hotchkiss RS, Karl IE. Reevaluation of the role of cellular hypoxia and bioenergetic faillure in sepsis. JAMA 1992;267:1503-1510. 


\section{CHAPTER 9}

Gastric $\mathrm{PgCO}_{2}$ and $\mathrm{Pg}_{3}-\mathrm{CO}_{2}$ gap are related

to D-lactale and not to L-lactate levels

in patients with septic shock

\section{Published in}

Intensive Care Med 2003; 29(2): 2081-2085 


\section{ABSTRACT}

Background: Intestinal ischaemia causes an increase in lactate production and gastric intramucosal carbon dioxide partial pressure $\left(\mathrm{PgCO}_{2}\right)$. However, no linear relationship between systemic L-lactate levels and gastric tonometry during intestinal ischaemia has been found, probably since L-lactate is rapidly cleared from the circulation by the liver. In contrast, the rate of D-lactate clearance from the circulation by the liver is considerably lower than that of L-lactate, 146 and D-lactate may therefore be more closely related to measurements of gastric tonometry than L-lactate values.

Design and setting: Prospective, observational study in a university-affiliated mixed intensive care unit.

Subjects. Twenty critically ill patients with septic shock.

Measurements and results. During the first $24 \mathrm{~h}$ of admission to the intensive care unit at least two blood samples were taken for $\mathrm{D}$ - and L-lactate measurements and arterial blood gases, simultaneously, gastric $\mathrm{PgCO}_{2}$ was measured using capnographic tonometry. The intramucosal-arterial $\mathrm{PCO}_{2}$ gap was calculated using gastric intramucosal $\mathrm{PgCO}_{2}$ and arterial $\mathrm{PCO}_{2}$ from arterial blood. D-lactate was significantly correlated to $\mathrm{PgCO}_{2}$ values and to the mucosal-arterial $\mathrm{PCO}_{2}$ gap. There was no relationship between $L-$ lactate and $\mathrm{PgCO}_{2}$ or the mucosal-arterial $\mathrm{PCO}_{2}$ gap. D-lactate and L-lactate values were significantly correlated.

Conclusions: During sepsis intestinal production of D-lactate is related to gastric intramucosal $\mathrm{PCO}_{2}$. No such relationship was found between L-lactate values and $\mathrm{PgCO}_{2}$. 


\section{INTRODUCTION}

Measurement of D-lactate has been suggested to be a better marker of splanchnic hypoperfusion than its isomer L-lactate. D-lactic acidosis in patients was originally described during short bowel syndrome $(1,2)$. Acidosis is caused by the production of D-lactic acid by bacterial fermentation in the gut lumen and its subsequent absorption into the blood (3). Although D-lactate dehydrogenase is present in certain lower animal species, in humans only L-lactate dehydrogenase is present. Therefore D-lactate cannot be metabolised in humans by this route and is thought to be metabolised more slowly than L-lactate (4).

During intestinal ischaemia the resident microbial flora of the intestine multiplies rapidly and soon overgrows the ischaemic segment (3). This bacterial proliferation occurs in conjunction with disruption of the mucosal layer of the gut. Both phenomena are well known features of gastro-intestinal dysfunction during septic shock. Both experimental and clinical studies have shown that acute intestinal ischaemia causes a rise in D-lactate levels in the systemic circulation $(3,4)$. Increased D-lactate levels were also found in experimental burn and acute pancreatitis models where levels of endotoxin correlated well with D-lactate levels (5).

All cells produce L-lactate as a product of glycolysis in a balanced equilibrium during inadequate oxygen supply (6). During hypoperfusion in the gut lactate is produced in excess and is released into the bloodstream. Thus both L-lactate and D-lactate are produced during ischaemia in increased amounts. However, D-lactate metabolism by the liver is considerably slower than that of L-lactate in the liver (7). D-lactate may therefore reflect the intestinal perfusion more closely than L-lactate.

This study was undertaken to evaluate the relationship of D-lactate and L-lactate with other measurements of splanchnic oxygenation. In this study gastric tonometry was used as a marker of splanchnic oxygenation. The value of gastric tonometry to detect splanchnic mucosal hypoperfusion has been demonstrated in previous studies $(8)$. Therefore, the hypothesis was that the levels of D-lactate are more closely related to the gastric intramucosal $\mathrm{PCO}_{2}$ than the L-lactate values.

\section{PATIENTS AND METHODS}

This prospective observational study was conducted in critically ill patients with septic shock. The institutional review board of the hospital approved the study. Written informed consent was obtained from the next of kin of each patient, since all patients were ventilated and sedated at the time of inclusion to the study. Critically ill patients with either systemic inflammatory response syndrome or sepsis as defined by the ACCP/SCCM consensus conference criteria were included (9). In the presence of clinically suspected or confirmed infection patients were defined as having sepsis. 
Patients with septic shock or severe systemüc inflammatory response syndrome accompanied by shock who were expected to succumb within the first 24 hrs were excluded, since we wanted to perform repeated measurements. Patients on dialysis were excluded, as were patients with septic shock based upon acute intestinal ischaemia were excluded.

During the first $24 \mathrm{hrs}$ (at least two) blood samples were taken from a catheter in the radial artery one at admission to the $\mathrm{CU}$ and one after $24 \mathrm{hrs}$. The collected sample for D-lactate determination was mixed with EDTA and immediately deproteinised with perchloric acid $\left(1 / 1\right.$ vol), centrifuged at $4^{\circ} \mathrm{C}$ at $1500 \mathrm{rpm}$ for $20 \mathrm{~min}$, after which the supernatant was removed and stored at $-70^{\circ} \mathrm{C}$ untill measurement.

After thawing, plasma was mixed with a nicotamide adenine dinucleotide (NAD)+-glycine-hydrazine solution to form pyruvate hydrazon with reduced NAD $(\mathrm{NADH})$ and $\mathrm{H}+$, at a $\mathrm{pH}$ of less then 9.0 . This reaction was initiated by adding $\mathrm{D}$-lactate dehydrogenase (LDH). The protein-free plasma was assayed for $\mathbb{D}$-lactate concentration by a previously described spectrophotometric method (10).

For quaritification of L-lactate in the serum the same technique was used as described above, however with replacement of the D-lactate standard and D-LDH by L-lactate and L-LDH. D-lactate levels lower than $0.11 \mathrm{mmol} / \mathrm{l}$ and L-lactate levels lower than $2.0 \mathrm{mmol} / \mathrm{l}$ were considered to be normal. Moreover, $\mathrm{Pg}-\mathrm{aCO}$ values above 1.0 were considered abnormal.

After inclusion patien's received a gastric tonometry catheter (14F, DatexOhmeda, Finland) for measurement of intramucosal $\mathrm{PgCO}_{2}$ and tonometryrelated measurements using the gas-automated capnograph (Tonocap TC-200, Datex-Ohmeda). Correct placement of these catheters was determined by radiographic investigations.

The gastric tonometry measurements $\left(\mathrm{PgCO}_{2}\right)$ were performed (simultaneously with the blood sampling) in triplicate at 5 -min intervals using the gas-automated capnography (Tonocap, Datex-Ohmeda). The $\mathrm{PaCO}_{2}$ values taken at the end of the triplicate measurement, were corrected for the central blood temperature measurements using the formulas as provided by the manufacturer (ABL 100 , Radiometer, Copenhagen). Gastric $\mathrm{PgCO}_{2}$ were measured after a calibration period of at least 15 min in order to reduce the dead-space effect of the balloon. Acid suppression using $\mathrm{H}_{2}$-blockers were given routinely using ranitidine. The gastric $\mathrm{Pg}_{\mathrm{aCO}}$ gap was calculated by subtracting $\mathrm{PaCO}_{2}$ from the gastric $\mathrm{PgCO}_{2}$.

For statistical analysis the SPSS software package (version 7.5) was used. Changes in mean values over time and between survivors and non-survivors were analysed using multivariate analysis of variance with within-factor time (repeated measures) and as between-factor survivor. The $\propto$ error values are reported in table 1, in which $\mathrm{P}_{\mathrm{T}}$ indicates the significance of repeated measures over time, $\mathrm{P}_{\mathrm{T} \times \mathrm{S}}$ the interaction of repeated measures and survivor status, and $P_{S}$ the effect of survival. Correlations between variablles were calculated using Pearson's correlation coefficient. The individual changes in D-lactate and the $\mathrm{Pg}-\mathrm{aCO}_{2}$ gap were compared to the mean and $95 \%$ confidence interval of the quadratic regression analysis. Statistical significance was set at $p<0.05$. 


\section{RESULTS}

In total, 20 patients with septic shock were assessed. The mean APACHE-II score was 18.5 (SE 2.4) with an expected mortality rate of $33 \%$. Survival in this patient group was $60 \%$. Mean D-lactate and L-lactate levels were $0.14(0.11)$ and 1.6 (1.4), respectively. The changes in all parameters during the study period in survivors and non-survivors are displayed in table 1.

Table 1

\begin{tabular}{|c|c|c|c|c|c|c|c|c|}
\hline Paramele & & \multicolumn{2}{|c|}{ Admission } & \multicolumn{2}{|c|}{ 24hrs. } & \multicolumn{3}{|c|}{ p-value } \\
\hline \multirow[t]{2}{*}{ MAP } & survivors & 78 & \pm 13 & 86 & \pm 16 & 0.01 & 0.03 & 0.6 \\
\hline & non-surviwors & 68 & \pm 6 & 81 & \pm 11 & & & \\
\hline \multirow[t]{2}{*}{$H R$} & survivors & 99 & \pm 16 & 98 & \pm 22 & 0.3 & 0.7 & 0.7 \\
\hline & non-5urviwors & 105 & \pm 17 & 96 & \pm 17 & & & \\
\hline \multirow[t]{2}{*}{$\mathrm{DO}_{2}$} & survivars & 660 & \pm 174 & 769 & \pm 236 & 0.07 & 0.3 & 1.0 \\
\hline & non-surviwors & 569 & \pm 172 & 654 & \pm 261 & & & \\
\hline \multirow[t]{2}{*}{$\mathrm{VO}_{2}$} & survivars & 120 & \pm 39 & 140 & \pm 44 & 0.06 & 0.2 & 0.5 \\
\hline & non-survivors & 165 & \pm 93 & 161 & \pm 70 & & & \\
\hline \multirow[t]{2}{*}{ SVRi } & survivors & 1423 & \pm 493 & 1129 & \pm 473 & 0.001 & 0.7 & 0.6 \\
\hline & non-5urvivors & $\# 293$ & \pm 647 & 1004 & \pm 244 & & & \\
\hline \multirow[t]{2}{*}{$\mathrm{PgCO}_{2}$} & surviwors & 4.0 & \pm 1.6 & 6.7 & \pm 2.9 & 0.007 & 0.2 & 0.7 \\
\hline & non-sunvivors & 5.3 & \pm 2.4 & 4.8 & \pm 1.0 & & & \\
\hline \multirow[t]{2}{*}{$\mathrm{Pg}_{-\mathrm{aCO}}$} & surviwors & 0.9 & \pm 1.2 & 1.5 & \pm 0.8 & 0.03 & 0.3 & 0.7 \\
\hline & non-survivors & 1.2 & \pm 0.5 & 1.6 & \pm 1.7 & & & \\
\hline \multirow[t]{2}{*}{ D-lactale } & survivors & 0.09 & \pm 0.11 & 0.11 & \pm 0.11 & 0.001 & 0.7 & 0.4 \\
\hline & non-survivors & 0.11 & \pm 0.14 & 0.24 & \pm 0.12 & & & \\
\hline \multirow[t]{2}{*}{ L-lactate } & Surwiwors & 1.0 & \pm 0.5 & 0.9 & \pm 0.5 & 0.01 & 0.6 & 0.8 \\
\hline & non-survivors & 2.3 & \pm 2.1 & 2.0 & \pm 1.9 & & & \\
\hline
\end{tabular}



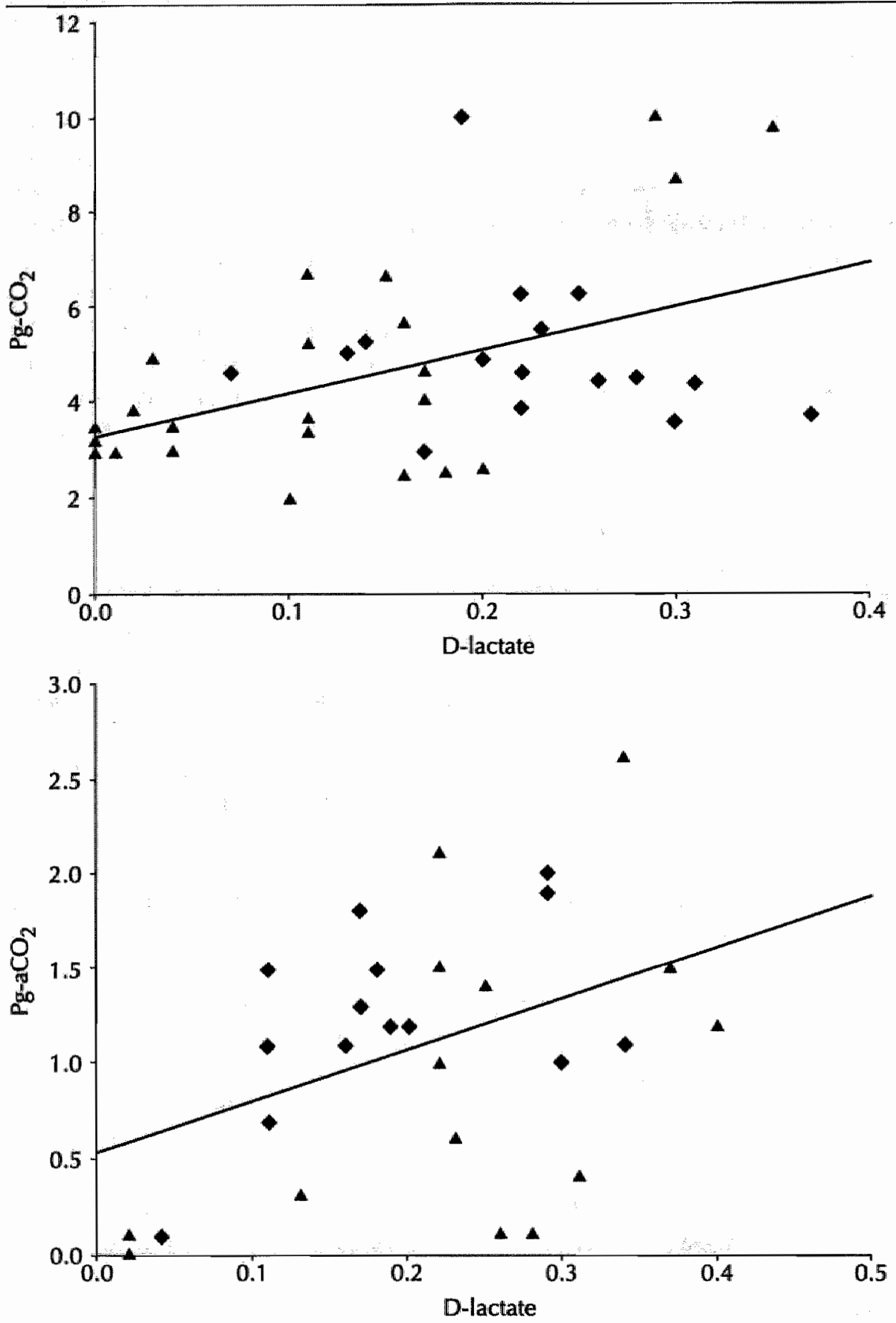

Correlation plots of the D-lactate concentration plotted against gastric intramucosal $\mathrm{PgCO}_{2}$ (abowe; $r=0.45, \mathrm{p}<0.0001$ ) and gastric $\mathrm{Pg}_{\mathrm{g}-\mathrm{aCO}}$ (below, $\mathrm{r}=0.44, \mathrm{p}=0.01$ ) for individual surviving (triangles) and non-surviving patients (diamonds) with septic shock. D-lactate concentration is in mmol/i, $\mathrm{PgCO}_{2}$ in $\mathrm{kPa}$.

Figure 1 Correlation between $\mathrm{D}$-lactate concentration and gastric intramucosal $\mathrm{PgCO}_{2}$ and $\mathrm{Pg}-\mathrm{aCO}_{2}$ in patients with septic shock 


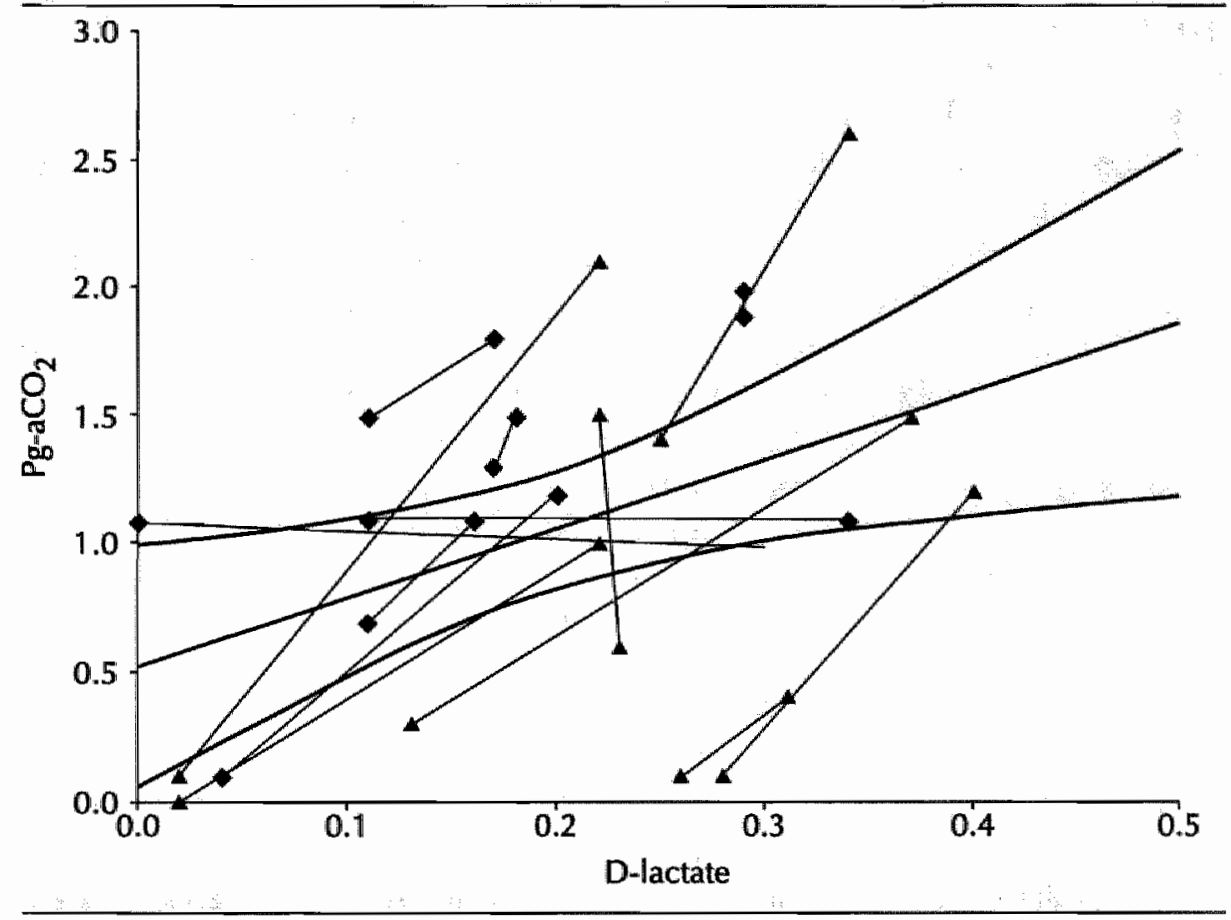

Correlation plot between D-lactate concentration and gastric $\mathrm{Pg}-\mathrm{aCO}$ gap showing individual changes in both parameters in patients with shock during the first $24 \mathrm{~h}$ after admission to the ICU. Lines Mean $\$ 1-95 \%$ confidence intervall of the quifdratic regression cunes, representing mean slope with the $95 \%$ confidence interval variation in the changes for individual patients. In 10 of 15 patients the slope of the changes were in the $95 \%$ area of the correlation coefficient.

Figure 2 Individual changes in D-lactate concentration and gastric intramucosal $\mathrm{Pg}_{\mathrm{g}} \mathrm{aCO}$ gap in patients with septic shock during $24 \mathrm{~h}$ period

D-llactate was significantly correlated to $\mathrm{PgCO}_{2}(r=0.45, \mathrm{p}<0.0001$; Figure 1) and to gastric $\mathrm{Pg}^{-a C O}(r=0.43, p=0.01)$. There was no significant relationship between L-lactate and $\mathrm{PgCO}_{2}(r=0.04, p=0.6)$ or the $\mathrm{Pg}-\mathrm{aCO}$, gap $(r=-0.09$, $p=0.6)$. D-lactate and L-lactate values were significantly intercorrelated $(r=0.53$, $p<0.0001$ ). No relationship was found between $D$-lactate levels and markers of global perfusion and oxygenation (mean arterial pressure, cardiac output, oxygen delivery, systemic vascular resistance) (data not shown). Fifteen of the 26 samples $(58 \%)$ of abnormal D-lactate values $(>0.11)$ were found in non-survivors, in contrast to one of $14(7 \%)$ in normal D-lactate samples $(p=0.02)$. These figures were $62 \%$ and $15 \%$ for $\mathrm{Pg}-\mathrm{aCO}$ gap (normal $\leq 1.0 ; \mathrm{p}=0.004$ ).

Figure 2 presents the changes over time in the relationship of D-lactate values to the $\mathrm{Pg}-\mathrm{aCO} \mathrm{CO}_{2}$ gap. For this the first and last samples from each patient were chosen. In 10 of 15 patients the slope of the changes were within the $95 \%$ confidence interval. 


\section{Discussion}

The main finding of this study was a significant correlation between the levels of D-lactate and the gastric $\mathrm{PgCO}_{2}$ and $\mathrm{Pg}-\mathrm{aCO}_{2}$ values while no such correlation was found between gastric $\mathrm{PrCO}_{2}, \mathrm{Pg}-\mathrm{aCO}$, and L-lactate levels. Disturbances in splanchnic $\mathrm{CO}_{2}$ values are an important determinant of outcome in critically ill patients (11-13). Systemic levels of L-lactate have been used as a marker for splanchnic oxygenation since L-lactate, as D-lactate, is produced in increased amounts during intestinal ischaemia. However, the interpretation is difficult, since the systemic concentration of L-lactate produced by the intestines is influenced by several factors, such as the rapid clearance of L-lactate by the liver. In contrast, uptake of D-lactate by the liver is probably slower than L-lactate and may therefore be more closely related to the level of intestinal ischaemia. This study was undertaken to investigate the relationship between the levels of D-lactate and the intramucosal $\mathrm{CO}_{2}$ values in septic shock.

There are a number of remarks which must be made when interpreting these observations. The site of D-lactic acid production is likely to be the colon since the colonic bacteria of humans metabolise carbohydrates that have not been metabolised in the small intestine (2). On the other hand intramucosal tonometry was performed in the stomach. During sepsis the heterogeneity of flow is a well-known phenomenon. However, the presence of a significant correlation between D-lactate and the measurement of the gastric tonometry may suggest that in general there is a reduced oxygen utilisation in the overall gut, despite regional differences.

Although both L-lactate and D-lactate are produced during splanchnic dysoxia (i.e. inadequate oxygen supply for the oxygen demand in the tissues, leading to an increased $\mathrm{CO}_{2}$ accumulation), only the $\mathrm{D}$-isomer is related to splanchnic luminal $\mathrm{CO}_{2}$ values as measured by tonometry. This is probably caused by the rapid metabolism of L-lactate in the liver (14). However, since no samples from the portal vein were taken, it was impossible to determine whether the L-lactate production in these patients is mainly from the hepatosplanchnic area. Several studies have reported a relationship between changes in portal vein lactate levels and the intramucosal pHi during splanchnic ischaemia (15). Other studies, however, have found no such relationship (16), although it has been suggested that changes in intramucosal $\mathrm{pHi}$ or $\mathrm{PrCO}_{2}$ occur at an earlier stage than release of lactate from the gut (7). Another possible explanation for the correlation between D-lactate and L-lactate levels is a general reduction in oxygenation both in the global and hepatosplanchnic circulation. This implies a relationship between D-lactate levels and parameters of global oxygenation. No relationship was found in this study between values of D-lactate and parameters of global perfusion.

When D-lactic acidosis was first described, it was thought that humans could not metabolise D-lactate. It is now clear that D-lactate can indeed be metabolised by humans although less rapidly so than L-lactate. In humans the enzyme D-LDH is lacking, but it has been hypothesized that d-2-hydroxy-acid-dehydrogenase, a mitochondrial enzyme converts D-lactate to pyruvate (17). Whether this is equally 
effective as $L$-lactate metabolism needs to be determined further. However, several studies have found a relatioship between increased systemic D-lactate levels, in comparison to L-lactate levels, and ischaemia-reperfusion of the gut or intestinal ischaemic vascular complications $(4,18)$.

The importance of a static correlation between $\mathrm{D}$-lactate and $\mathrm{PgCO}_{2}$ values may be questioned. Indeed, from a pathophysiological point of view, the relationship of changes in the $\mathrm{Pg}-\mathrm{aCO}_{2}$ gap and D-lactate in individual patients is more relevant than a correlation in a pooled set of data. Our data suggest that there is not only a positive correlation between the $\mathrm{Pg}-\mathrm{aCO}_{2}$ gap and D-lactate values, but that the positive correlation can be found in the majority of patients. This may suggest that changes in mucosal $\mathrm{CO}_{2}$ production are a determining factor for the production of D-lactate. The relationship of the abnormal values with survival also indicates the importance of these findings.

From the data presented, the observation can be made that a considerable number of patients have normal $\mathrm{Pg}-\mathrm{aCO}_{2}$ gap values with above-normal D-lactate values. This suggests that preserved macrocirculatory perfusion in the gut does not systematically protect against mucosal dysoxia, as sepsis can induce microcirculatory impairment. A study by Tugtekin et al. (19) observed that $50 \%$ of the microcirculation should be abnormally perfused, as assessed by intravital microscopy, to observe increase in the $\mathrm{Pg}-\mathrm{aCO}_{2}$ gap. This may in part explain why some patients had increased D-lactate levels related to normal $\mathrm{Pg}-\mathrm{aCO}_{2}$ values.

In conclusion, we report for the first time that D-lactate levels are increased in septic shock patients. Moreover, these D-lactate values were correlated with gastric intramucosal $\mathrm{PgCO}_{2}$ and mucosal-arterial $\mathrm{PCO}_{2}$ values. D-lactate seems pathophysiologically to be a better marker of disturbed splanchnic luminal $\mathrm{CO}_{2}$ production than L-lactate in septic patients. For the detection of non-corrected splanchnic disturbances in oxygenation during resuscitation of septic patients D-lactate, instead of L-lactate, seems to be important. 


\section{REFERENCES}

1. Oh MS, Phelps $\mathrm{KR}_{s}$ Traube M, Barbosa-Saldivar $\mathrm{L}$, Boxhill $\mathrm{C}$, Carroll HI D-lactic acidosis in a man with the short-bowel syndrome. New Eng I Med 1979;301:249-52.

2. Hove $H$, Mortensen PB. Colonic lactate metabolism and D-lactic acidosis. Dig Dis Sci 1995;40: 320-30.

3. Murray M), Barbose II, Cobb CF. Serum D(-)-lactate levels as a predictor of acute intestinal ischemia in a rat model. I Surg Res 1993;54:507-9.

4. Murray M), Conze MD, Nowak LR, Cobb CF. Serim D(-)-lactate levels as an aid to diagnosing acute intestinal ischemia. Am J Surg 1994; 167:575-8.

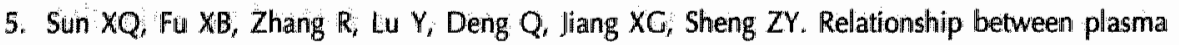
$\mathrm{D}(-)$-lactate and intestinal damage after severe injuries in rats. World / Gastroenterol $2001 ; 7$ : $555-8$.

6. Brooks $C A$ Lactate production under fully aerobic conditionis: The lactate shuttle during rest and exercise. Fed Proc 1986:45:2924-9.

7. Takala I, Uusaro A, Parviainen I, Ruokonen E. Lactate metabolism and regional lactate exchange after cardliac surgery. New Horiz 1996;4:483-91.

8. Elizalde Jl, Hernandez $C_{\text {, }}$ llach J, Monton C, Bordas JM, Pique JM, Torres A. Gastric intramucosal acidosis in mechanically ventilated patients: role of mucosal blood flow. Crit Care Med 1998;26:827-32.

9. Bone RC, Balk RA, Cerra FB, Dellinger RPh, Fein AM, Knaus WA, Schein RA, Sibbald W]. Definitions for sepsis and organ failure and guidlelines for the use of innovative therapies in sepsis. Chest 1992; 101:1644-55.

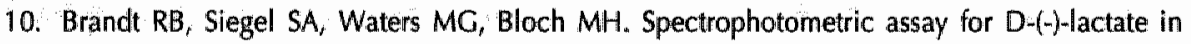
plasma. Anal Biochem 1980;102:39-46.

11. Trinder $T \mathrm{~J}$, Lavery $\mathrm{GG}$, Fee $\mathrm{IP}$, Lowry KG Low gastric intramucosal $\mathrm{pH}$ : incidence and significance in intensive care patients. Anaesth Intensive Care 1995;23:315-21.

12. Poeze $M$; Takala J, Greve JWM, Ramsay $G$. Pre-operative tonometry is predictive for mortality and morbidity in high-risk surgical patients. Intensive Care Med 2000;26:1272-81.

13. Gomersall $C D$, Joynt $G M$, Ho KM, Young RJ, Buckley TA, Oh TE. Gastric tonometry and prediction of outcome in the critically ill. Arterial to intramucosal $\mathrm{pH}$ gradient and carbon dioxide gradient. Anaesthesia 1997;52:619-23.

14. Smith SM, Eng HK, Buccini F. Use of D-lactic acid measurement in the diagnosis of bacterial infections. IInfect Dis 1986;154:658-64.

15. Heino A, Hartikainen J, Merasto ME, Alhava E, Takala J. Systemic and regional $\mathrm{pCO}_{2}$ gradients as markers of intestinal ischaemia. Intensive Care Med 1998;24:599-604.

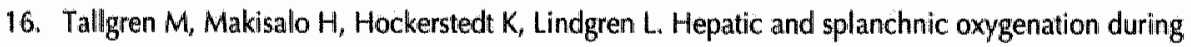
liver transplantation. Crit Care Med 1999;27:2383-8.

17. Uribarri $\mathrm{H}_{\text {, }} \mathrm{OhMS}$, Carroll HI. D-lactic acidosis. A review of clinical presentation, biochemical features, and pathophysiological mechanisms. Medicine (Baltimore) 1998;77:73-82.

18. Poeze $M$, Froon AHM, Greve JWM, Ramsay G. D-llactate as an early marker of intestinal ischaemia after ruptured abdominal aneurysm repair. Br J Surg 1998;85:1221-4.

19. Tugtekin IF, Radermacher $P$, Theisen $M$, Matejovic $M$, Stehr $A$, Ploner $F$, Matura $K_{r}$ Ince $C$, Georgieff $M$, Traeger $K$. Increased ileal-mucosal-arterial $P C O_{2}$ gap is associated with impaired villus microcirculation in endotoxic pigs. Intensive Care Med 2001;27:757-66. 


\section{CHAPTER 10}

Pre-operalive tonometry is predictive

for mortality and morbidity

in high-risk surgical patients

Published in

Intensive Care Med, 26(9):2000;1272-1281 


\section{ABSTRACT}

Objective. To determine whether a) pre-operative measurement of gastric intramucosal $\mathrm{pHi}$ is predictive for mortality and morbidity in high-risk surgical patients and b) peri-operative improvement of global oxygen delivery $\left(\mathrm{DO}_{2}\right)$ with fluids and dopexamine leads to increased gastric $\mathrm{pHi}$ and either improved global perfusion or improved splanchnic perfusion is related to the prevention of multiple organ failure (MOF).

156 Design. Retrospective analysis of a double-blind, placebo controlled, randomised study.

Setting: General intensive care units from 14 hospitals.

Patients. 286 high-risk surgical patients.

Interventions: Swan-Ganz and tonometer catheter placement; patients were stabilised pre-operatively using fluids, blood and/or oxygen to preset goals before receiving placebo or two doses of dopexamine $(0.5$ or $2.0 \mu \mathrm{g} / \mathrm{kg} / \mathrm{min})$ perioperatively.

Measurements and results: Haemodynamic assessment (including $\mathrm{DO}_{2}$ and oxygen consumption $\left(\mathrm{VO}_{2}\right)$ ) was performed togheter with measurement of gastric mucosal pHi pre-operatively and directly, 2, 6, 12, 24, and 36hrs post-operatively. Patients were divided pre-operatively into two subgroups based on the optimal cut-off value for mortality of the first $\mathrm{pHi}$ measurement after induction of anaesthesia as calculated by a receiver operator characteristic curve analysis - low pHi group $(<7.35)$ and normal $\mathrm{pHi}(\geq 7.35$ ). Mortality in low $\mathrm{pHi}$ was higher than in the normal $\mathrm{pHi}$ group ( 16.8 vs. $2.3 \%$; $=0.0001)$. In the normal $p H i$ group dopexamine, which was given prior to the first $\mathrm{pHi}$ measurement, had no effect on $\mathrm{pHi}$, while $\mathrm{DO}_{2}$ increased significantly. In this group MOF-score and number of patients with MOF remained similar between the treatment subgroups. In the low pHi group gastric $\mathrm{pHi}$ increased significantly during dopexamine infusion $(p=0.008)$, despite the lack of an increase in $\mathrm{DO}_{2}$ and $\mathrm{VO}_{2}$. In this group the MOF-score and the number of patients developing MOF decreased significantly using dopexamine $(p=0.04)$. In both groups bicarbonate levels remained similar between the treatment subgroups.

Conclusions: In high-risk surgical patients pre-operative measurement of $\mathrm{pHi}$ was predictive for mortality. The peri-operative response of $\mathrm{pHi}$ to dopexamine seemed to be dependent on pre-operative gastric $\mathrm{pHi}$. 


\section{INTRODUCTION}

Multiple organ failure (MOF) is the major cause of late death and serious morbidity following surgery $(1,2)$. High-risk surgical patients are especially prone to the development of multiple organ failure (3).

One of the current hypotheses for cellular and organ dysfunction is based on the presence of unrecognised hypovolaemia, leading to regional dysoxia, which could be the main pathophysiological disturbance leading to MOF (4). A normal compensatory mechanism to hypovolaemia is vasoconstriction; particulary in the skin and gut. Shoemaker et al. found that in critically ill surgical patients increasing the oxygen delivery $\left(\mathrm{DO}_{2}\right)$ peri-operatively to values obtained in surviving surgical patients decreased the organ-failure related mortality (3). This has been confirmed by others in postoperative patients, as well as in patients after trauma, whereas patients with sepsis had no benefit from achieving supranormal values for oxygen transport (5-9). Moreover, global hypovolaemia is a potent cause of gut mucosal hypoperfusion and the latter seems to be related to the development of MOF (10). Correction of the gastric mucosal acidosis has been shown to reduce the incidence of organ failure in postoperative patients (11). Thus, aiming peri-operatively at supranormal values for both global and regional perfusion parameters would be expected to reduce the incidence of postoperative organ failure.

In two trials using dopexamine and plasma volume expansion as a means of achieving supranormal values of $\mathrm{DO}_{2}$ and oxygen consumption $\left(\mathrm{VO}_{2}\right)$ Boyd et al. and Wilson et al. reduced the mortality in postoperative patients $(6,12-15)$. However, Uusaro showed that after cardiac surgery splanchnic perfusion may remain inadequate despite correction of global haemodynamics (16).

Clearly global and regional haemodynamics do not always change in parallel $(16,17)$. Thus, improving global perfusion does not necessarily lead to improvement of regional perfusion. Our hypothesis was that improving global oxygen transport is not necessarily associated with improvement in the gastric mucosal $\mathrm{pHi}$. The database from the European multicenter study on dopexamine was used to test this hypothesis (18). We report an analysis of peri-operative changes in regional and global perfusion in high-risk surgical patients in whom dopexamine, in addition to plasma volume expansion, was used to optimise the peri-operative haemodynamic status.

\section{PATIENTS AND METHODS}

Institutional ethics committee approval from all participating centres was obtained and patients or close relatives gave informed consent for enrolment in the study. The study has been conducted according to the principles esthablished in Helsinki. Patients undergoing non-vascular, abdominal surgery of an expected duration of 
greater than or equal to $1.5 \mathrm{~h}$, and fulfilling one or more of the high-risk criteria devised by Shoemaker (19) were randomised to one of three treatment groups. A total of 412 patients were included across 14 centres during a 20 -month period from November 1994 to June 1996. The data for this analysis were obtained from the European multicenter study on dopexamine in high-risk abdominal surgical patients (18).

\section{Peri-operative treatment and assessment}

Patients identified as 'high-risk' were admitted preoperatively to the intensive care unit (ICU). At this time patients received a pulmonary artery catheter and an intraarterial catheter. During stabilisation fluids, blood products and/or oxygen were given if patients had a wedge pressure less than $10 \mathrm{mmHg}$, a cardiac index than $2.5 \mathrm{l} / \mathrm{min} / \mathrm{m}^{2}$, mean arterial pressure less than $70 \mathrm{mmHg}$, a haemoglobin concentration of less than $10 \mathrm{~g} / \mathrm{dl}$ or arterial oxygen saturation $\left(\mathrm{SaO}_{2}\right)$ less than $94 \%$. After stabilisation, patients were randomised in a blinded fashion to placebo or one of two doses of dopexamine $(0.5$ or $2.0 \mu \mathrm{g} / \mathrm{kg} / \mathrm{min})$.

In all centers, patients received a nasogastric tonometry tube, unless contraindicated, after induction of anaesthesia and saline installed in the balloon for at least half an hour. The salline was removed after this equilibration time and analysed on a blood gas analyser system. The variability of the different blood gas analysers among the centers was assessed by testing different standard saline samples with known $\mathrm{PCO}_{2}$. Standardised $\mathrm{PCO}_{2}$ variability was not significantly different among the centres. The mean coefficient of variation among the different centers was $1.57 \%$. The measurement of $\mathrm{pHi}$ was used as an observational tool only and treatment was not directly aimed at changing the intramucosal $\mathrm{pH}$. The use of acid suppression therapy was not protocolised in the study.

The dopexamine infusion was maintained during surgery and for the following 24hrs. Fluids were given throughout this period as required to maintain filling pressures, as defined by the above-mentioned criteria. In addition to haemodynamic measurements, gastric intramucosal $\mathrm{pHi}$ was determined at regular intervals (pre-operatively, directly post-operatively, $2 \mathrm{~h}, 6,12,24$ and 30 to $36 \mathrm{hrs}$ post-operatively). The dopexamine treatment was started in advance of the first pHi measurement. The actual median time between the start of the stabilisation and the first pre-operative pHil measurement was $3 \mathrm{~h} 33 \mathrm{~min}$. The median time between the start of the stabilisation and the first post-operative pHi measurment was $5 \mathrm{~h} 33 \mathrm{~min}$. After this time-point the $2 \mathrm{~h}$ post-operative time-point was determined after a median time of $2 \mathrm{~h}$. For the $6,12,24$ and $30-36 \mathrm{hrs}$ post-operative time-point these figures were $6 \mathrm{~h}, 11 \mathrm{~h} 55 \mathrm{~min}, 23 \mathrm{~h} 45 \mathrm{~min}$, and $35 \mathrm{~h} 35 \mathrm{~min}$, respectively. 


\section{Patient follow-up}

Post-operatively patients were readmitted to the ICU. The same goals as set preoperatively were used post-operatively in order to optimise the haemodynamic condition of the patient. Any necessary treatment was permitted. The Acute Physiology and Chronic Health Evaluation (APACHE)-Il and MOF-scores (modified from Goris et al.) were calculated (20). Patients having a MOF-score above 4 were defined as having multiple organ failure. At $24 \mathrm{~h}$ the infusion rate of dopexamine or placebo was stepwise down-titrated. After 30-36 h patients could be discharged from the ICU should their condition no longer require intensive care management. After discharge from the ICU, patients were monitored for 28 days or until final hospital discharge for morbidity and mortality. Organ failure was assessed by the MOF-score on day 2-6, days 13,20 and 27.

\section{Patient sub-analysis}

A retrospective analysis was performed on those patients in whom a pre-operative (after induction of anaesthesia) gastric pHi could be obtained ( $n=286$ ). Patients were divided into two subgroups, based upon the optimal cut-off point value as analysed by a analysis of the receiver operator characteristic ( $R O C$ ) curve (see statistics and results section) - 'normal $\mathrm{pHi}^{\prime}$ and 'low pHi'. A pHi below 7.35 was considered in this population to be the optimal indication of a significant mucosal acidosis. Previously, $\mathrm{pHi}$ values below this level have been demonstrated to be related with an increased risk of MOF and mortality (21-23).

\section{Statistical analysis}

Statistical analysis was performed testing using SPSS version 7.5. Data are presented as mean \pm standard deviation, or as absolute numbers with percentages and compared using Student's t-test or by chi-square test.

The predictive values of the $\mathrm{pHi}$ measurements for mortality obtained pre-operatively and during the total peri-operative period, were plotted using ROC curves and compared using a statistical method previously described $(24,25)$. The areas under the ROC curve represent the probability that a randomly chosen individual deceased patient is correctly assessed for having a greater risk of death than a randomly chosen non-deceased individual patient. Thus, this gives an indication of the prognostic power in individual patients. The optimal cut-off value for the pre-operative value for mortality was calculated from the ROC curve analysis as that point with the greatest combined sensitivity and specificity. This cut-off value was used to divide the population into two subgroups (26). The distribution of patients in the low and normal $\mathrm{pHi}$ group among the different participating centers was compared using a chi-square test.

Since dopexamine was started prior to the first $\mathrm{pHi}$ measurement, pre-operative $\mathrm{pHi}$ values were first compared among the different treatment groups, using one- 
way ANOVA with Bonferoni post hoc analysis. Secondly, global and regional haemodynamic data and MOF-scores between treatment groups, with the low and normal $\mathrm{pHi}$ group analysed seperately, were compared by a multiple-way analysis of variance (ANOVA), with one within-factor: time (repeated measures) and one between-factor: treatment with dopexamine (including three levels). Where appropriate, the last observation carried forward principle was used to replace missing data. The mean percentage of missing values was $6.2 \%$ (SD 3.4\%). Error values of the multiple-way ANOVA are reported in table 2 and 3 as time-effect, to indicate the significance of the within-subject analysis and treatment effect (see also figure 2 and 3) to indicate the significance of the interaction between repeated measures times dopexamine treatment. The Bonferoni post hoc analysis was performed to determine which particular point in time differed significantly between the three treatment groups and the significance at a level of 0.05 is reported as an asterix in the tables and figures.

The combined and independent effects of the haemodynamic variables, which were significant on chi-square or multiple-way ANOVA analysis, were analysed using a logistic non-parametric multiple regression model (backward conditional). A p-value $<0.05$ was considered statistically significant.

\section{RESULTS}

\section{Overall clinical data}

Of the 412 patients in the overall study a total of 286 patients, in whom preoperative gastric tonometry was measured, were included in the analysis. The remaining patients had no gastric tonometer placed (due to gastro/esophageal surgery, $n=98)$ or the pre-operative measurement was not performed $(n=28)$. Overall outcome between the patients included in the substudy and the patients excluded was comparable.

\section{Optimal gastric intramucosal pHi cut-off value}

The area under the ROC curve for the pre-operative measurement of pHi was 0.79 (Figure 1). The area under the ROC-curve for all time-points combined was $0.69(\mathrm{SE}=0.013 ; \mathrm{Cl} 2.5-97.5 \%=0.67-0.72)$, and significantly lower than the area under the ROC-curve for the pre-operative measurement $(p=0.04)$. The optimal cut-off point for the pre-operatively measured $\mathrm{pHi}$ was 7.35 with a sensitivity of $86.4 \%$ and a specificity of $63.6 \%$. 
Table 1 Patient demographics

\begin{tabular}{|c|c|c|c|}
\hline Variables & Normal pHi group & Low pHi group & pevalue \\
\hline Number & 172 & 113 & \\
\hline Age (years) & $62 \pm 14$ & $63 \pm 14$ & 0.6 \\
\hline Gender ( $\%$ male $)$ & 54.7 & 58.4 & 0.5 \\
\hline Height $(\mathrm{cm})$ & $167 \pm 9$ & $168 \pm 11$ & 0.4 \\
\hline Weight: (kg) & $69 \pm 13$ & $72 \pm 16$ & 0.2 \\
\hline \multicolumn{4}{|l|}{ Diagnosis $(n)^{a}$} \\
\hline Carcinoma & 96 & 56 & \\
\hline Malignant neoplasm & 17 & 12 & \\
\hline Secondary malignancy & 15 & 3 & \\
\hline Benign neoplasm & 4 & 2 & \\
\hline Perilonitis & 8 & 11 & \\
\hline Pancreatitis & 7 & 5 & \\
\hline Enteritis/colitis & 16 & 11 & \\
\hline Cholecystitis/cholangitis & 1 & 2 & \\
\hline Duodenal ulcer & 3 & 3 & \\
\hline Other diagnoses & 13 & 14 & 0.2 \\
\hline \multicolumn{4}{|l|}{ Procedures $(n)^{\mathrm{a}}$} \\
\hline Resection intestine & 94 & 77 & \\
\hline Splenectomy & 3 & 1 & \\
\hline Biliary tract procedure & 5 & 6 & \\
\hline Hepatectomy & 24 & 7 & \\
\hline Pancreasresection & 35 & 19 & \\
\hline Uninary tract procedure & 3 & 4 & \\
\hline Genital tract procedure & 4 & 3 & \\
\hline Other procedures & 21 & 21 & 0.6 \\
\hline Acute/elective & 36172 & $28 / 113$ & 0.4 \\
\hline
\end{tabular}

apatients may have more than one diagnosis or procedure performed. Values are shown as mean \pm standard deviation. 


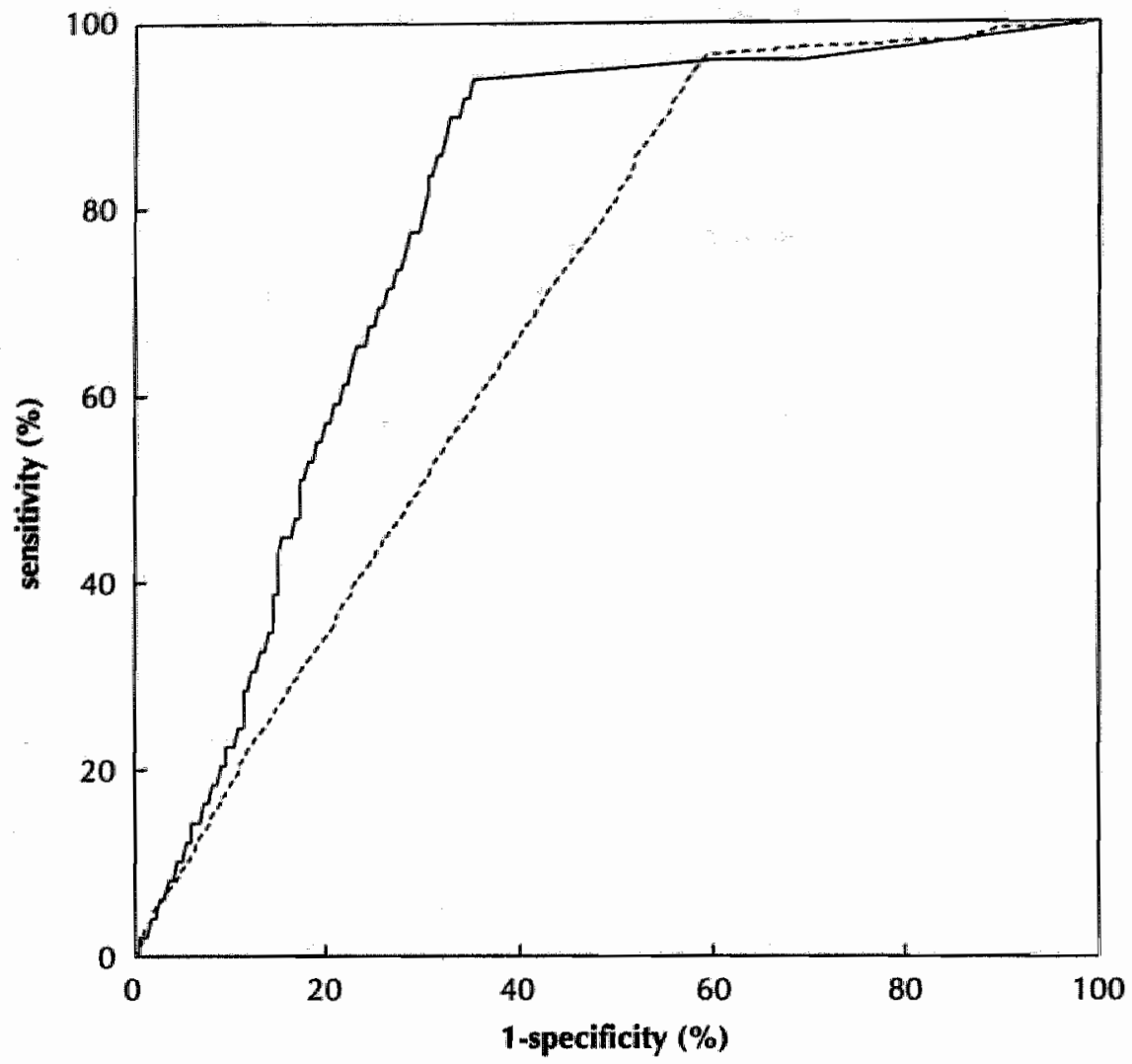

The maximal value for the area under the ROC-curve is 1 . This represents the best identification of the randomly chosen sick patient having the disease compared to a randomly chosen non-diseased patient. The area under the ROC-curve from the presoperative $\mathrm{pH}$ (-_- ) was signilicantly larger than the area under the curve from the peri-operatively (---) measured pH Hi $(p=0.04)$.

Figure 1 Predictive value of pre-operative and peri-operative pHi as assessed by receiver operator characteristic (ROC) curves

The low pHi group (113 patients, with a pre-operative gastric pHi mean of 7.26 (SD 0.08)) had a significantly higher mortality than the normal pHi group (172 patients, with a mean pHi of 7.47 (SD 0.10)) (16.8 vs. $2.3 \%$; $\mathrm{p}=0.0001)$. The mortality rates for the different $\mathrm{pHi}$ values are depicted in Figure 2 . The mortality rates for pre-operatively measured $\mathrm{pHi}$ showed a sudden increase in the group of patients with values below 7.35 compared to those with pre-operative pHi levels above 7.35 . 


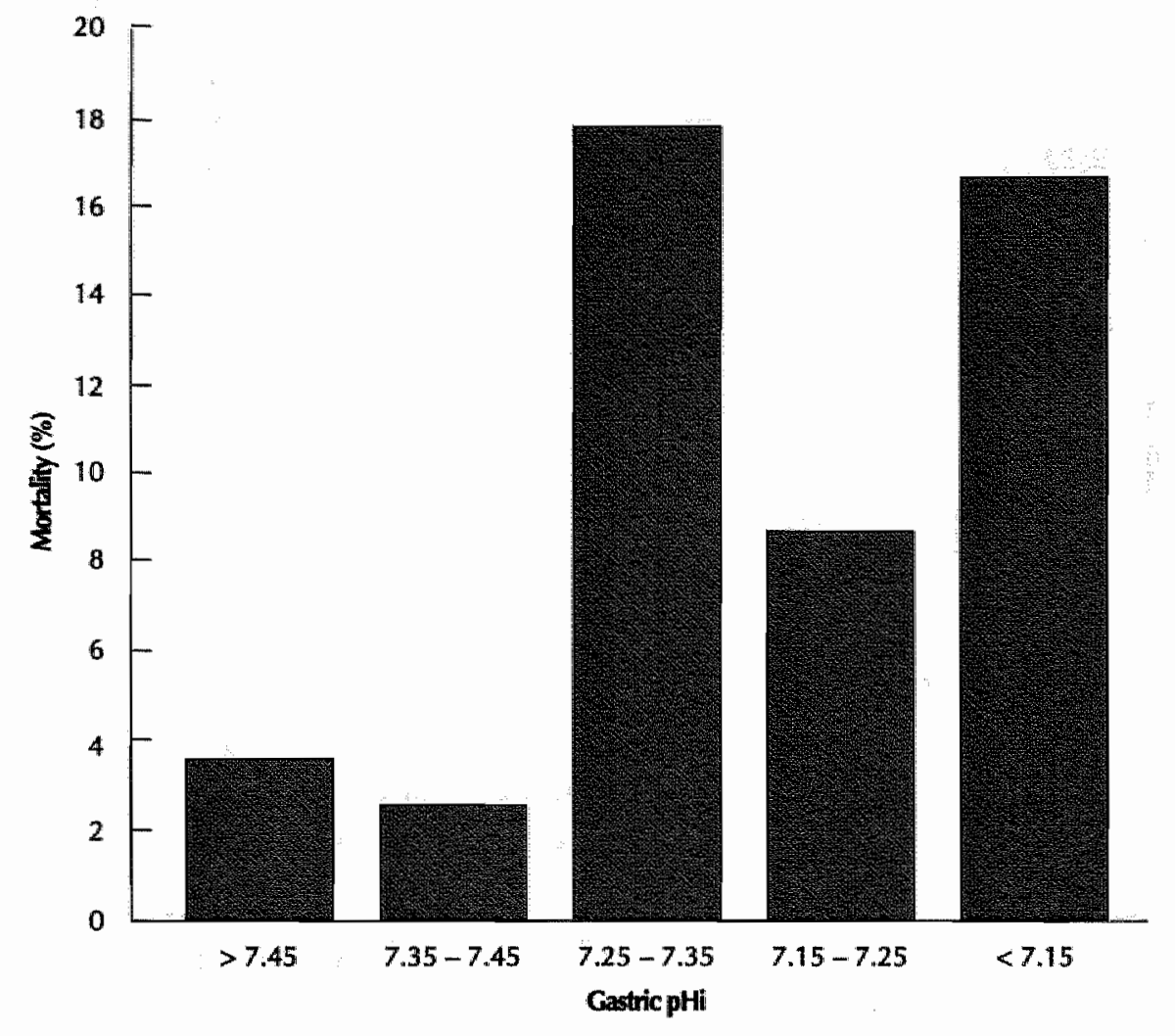

Data are presented as percentages. Mortality associated with the pre-operatively pHi showed a biphasic patten. $p=0.0001$, mortality for patients with a pre-operative $\mathrm{pH} i<7.35$ versus mortality for patients with a pre-operative $\mathrm{pHi} Z$ 7.35 by Chi-square test.

Figure 2 Mortality figures by gastric pHi

\section{Clinical data in low and normal pHi group}

There were no differences with respect to age, diagnosis, and operation type between the low and normal pHli groups. Table 1 shows the demographics of patients in these two subgroups. The distribution of patients in the low and normal pHli group among the different participating centers was not different $(p=0.3)$. The distribution of preoperative $\mathrm{pHi}$ values was similar in all participating centers.

In the group with a low pHi pre-operatively 39,37 , and 37 patients were randomised to receive placebo, 0.5 and $2.0 \mu \mathrm{g} / \mathrm{kg} / \mathrm{min}$ dopexamine, respectively. In the normal $\mathrm{pHi}$ group, 58 patients were randomised to receive placebo, 61 patients were randomised to receive $0.5 \mu \mathrm{g} / \mathrm{kg} / \mathrm{min}$ dopexamine, and 53 to receive $2.0 \mu \mathrm{g} / \mathrm{kg} / \mathrm{min}$ dopexamine. 


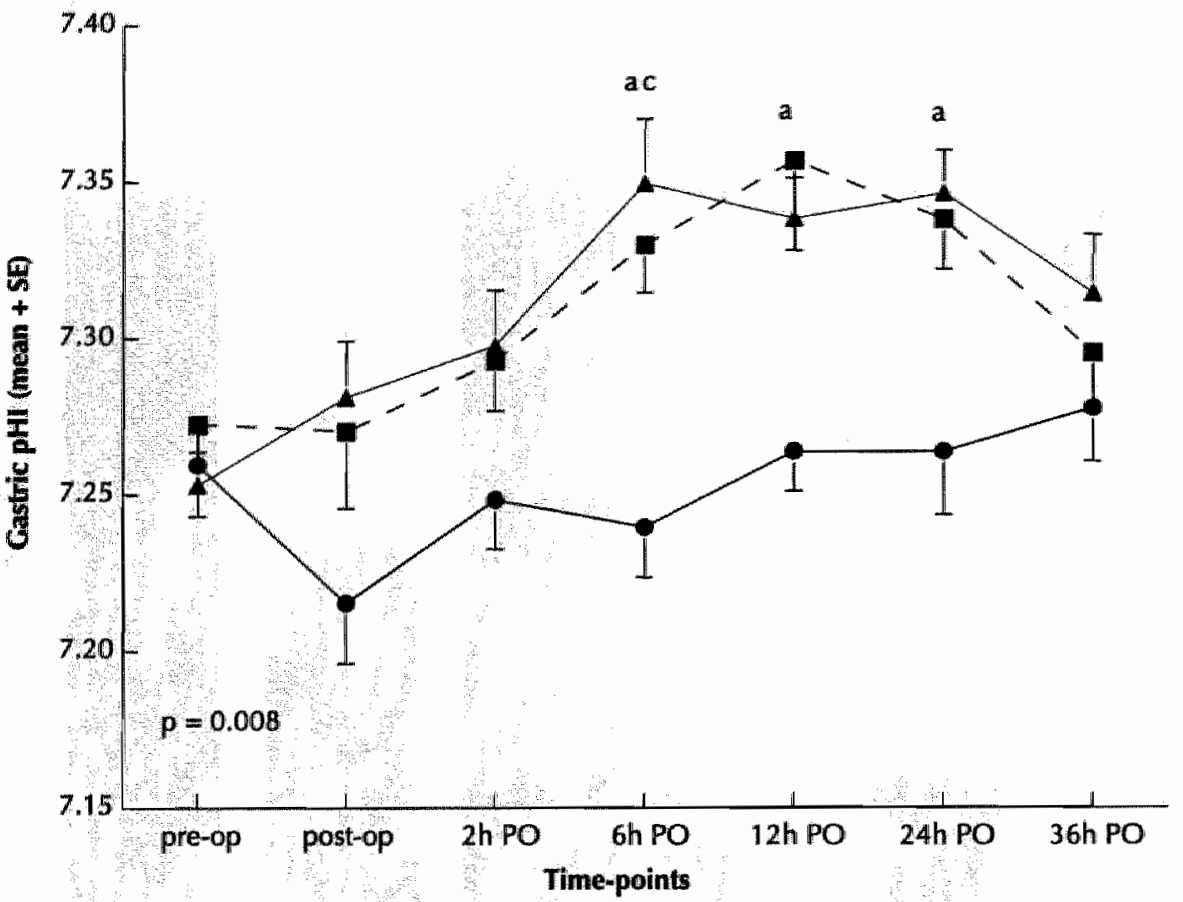

Data are presented as mean \pm standard error. Data were compared using repeated measures ANOVA (p-value indicales the interaction between the repeated lime-point $x$ dopexamine treatment; treatment-effect). Bonferroni correction was carried out

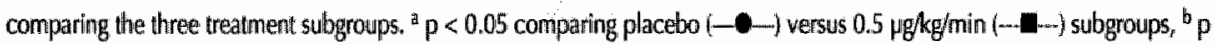
$<0.05$ comparing 0.5 and $2.0(\cdots-\cdots \cdot) \mu g / k g / m i n$ subgroups, ${ }^{c} p<0.05$ comparing placebo versus $2.0 \mu g / k g / m i n$ subgroups.

Figure 3 Peri-operative gastric pHi in patients with low pre-operative pHi treated with dopexamine

Although $\mathrm{H}_{2}$-blockade was not routinely used in the study, the majority of patients received acid suppression therapy during surgery. The number of patients receiving acid suppression therapy was equally distributed among the normal and the low pHi group $(91 / 172$ versus $65 / 113, p=0.44)$. Moreover, the mean pre-operative $\mathrm{pHi}$ value was similar in the patients with and without acid suppression therapy (7.384 (SD 0.139) versus 7.402 (SD 0.137 ), $p=0.31$ ).

\section{Changes in pHi during dopexamine infusion}

The first $\mathrm{pHi}$ measurement, performed after induction of anaesthesia, showed no differences between the treatment groups (placebo 7.398 (SD 0.157), $0.5 \mu \mathrm{g} / \mathrm{kg} / \mathrm{min}$ 7.390 (SD 0.117), $2.0 \mu \mathrm{g} / \mathrm{kg} / \mathrm{min} 7.390$ (SD 0.140); $p=0.27$, Bonferoni-analysis, $p=1.0$ ) 


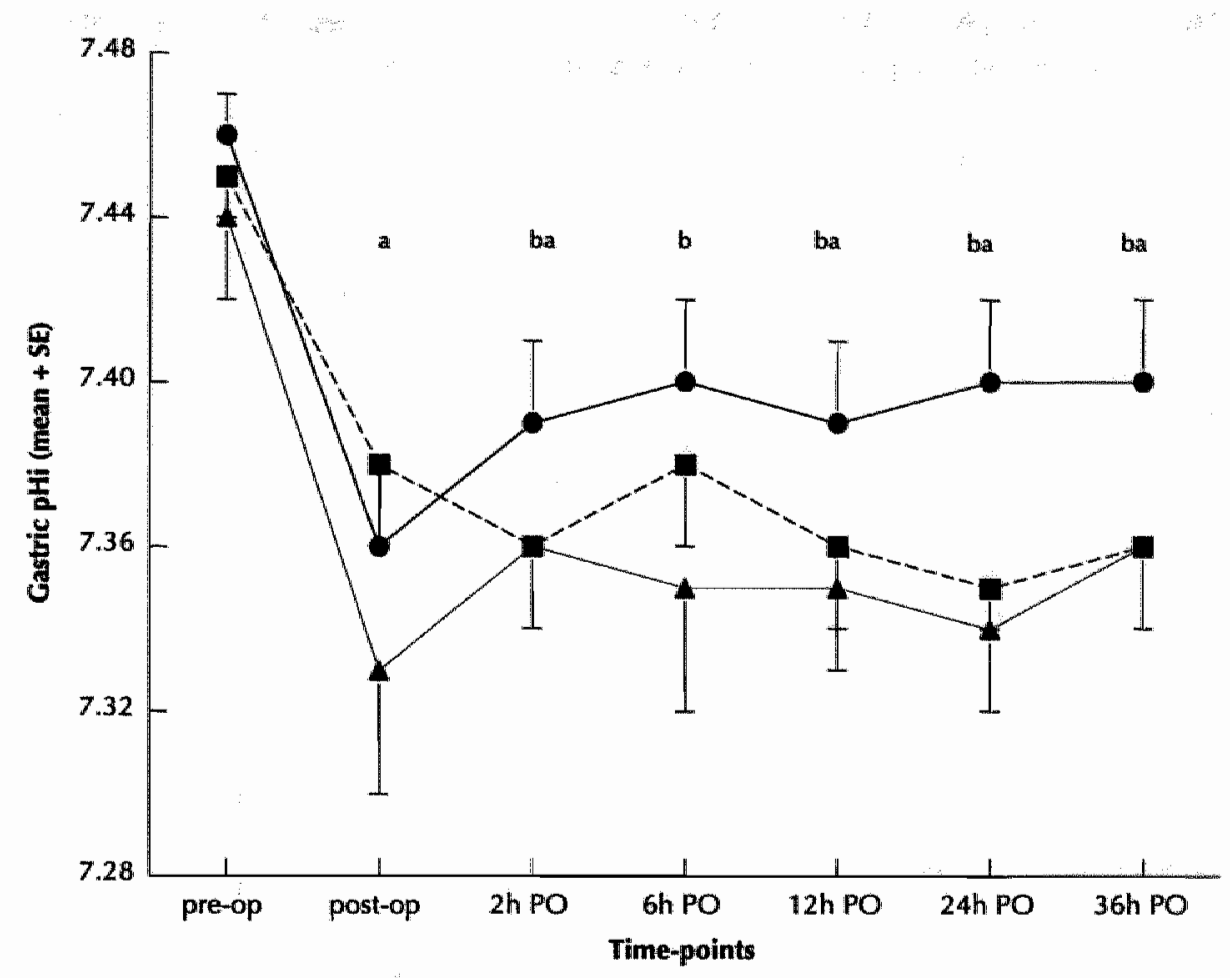

Data are presented as mean \pm standard error. Data were compared using repeated measures ANOVA ( $p$-value indicates the interaction between the repeated time-point $x$ dopexamine treatment; treatment-effect). Bonferroni correction was carried out to compare the three treatment subgroups. ${ }^{\text {a }} p<0.05$ comparing placebo $(-\rightarrow)$ versus $2.0 \mu \mathrm{g} / \mathrm{kg} / \mathrm{min}(\cdots . \mathrm{A} \cdot \cdots)$ subgroups; $0.5 \mathrm{Hg} / \mathrm{kg} / \mathrm{min}(-\cdots)$

Figure 4 Peri-operative gastric pHi in patients with mormal pre-operative pHi treated with dopexamine

In the total group of patients with a low pHi pre-operatively, the pHi increased steadily following surgery, but remained below 7.35. However, the $\mathrm{pHi}$ in the placebo treated patients in the low $\mathrm{pHi}$ group did not change significantly post-operatively compared to the pre-operative value $(p=0.13)$. In $84 \%$ of the placebo treated patients in the low $\mathrm{pHi}$ group, the $\mathrm{pHi}$ decreased below 7.35 during at least one time-point. These percentages were 64 and $65 \%$ for patients in the 0.5 and $2.0 \mu \mathrm{g} / \mathrm{kg} / \mathrm{min}$ dopexamine group, respectively.

In the normal pHi group, gastric pHi dropped significantly $(\mathrm{p}=0.005)$ in the placebo treated patients during surgery and remained stable thereafter. Despite the fall between pre-operative and post-operative values, the mean postoperative values remained within normal limits (> 7.35). However, $41 \%$ of the placebo-treated patients in the normal $\mathrm{pHi}$ group had a temporary drop of $\mathrm{pHi}$ below 7.35 during at 
Table 2 Peri-operative changes in global hemodynamic perfusion during treatment with dopexamine in patients with a normal $\mathrm{pHi}$ pre-operatively

\begin{tabular}{|c|c|c|c|c|c|c|c|c|c|c|c|c|c|}
\hline \multicolumn{2}{|c|}{ Subgroups } & \multicolumn{2}{|c|}{ Pre-dopex } & \multicolumn{2}{|c|}{ Preop } & \multicolumn{2}{|c|}{ Post-op (PO) } & \multicolumn{2}{|c|}{$24 \mathrm{hPO}$} & \multicolumn{2}{|c|}{36 ค PO } & \multirow[t]{2}{*}{$P_{1}$} & \multirow[t]{2}{*}{$p_{2}$} \\
\hline \multirow[t]{3}{*}{$\mathrm{DO}_{2}$} & plac & 474 & \pm 20 & 456 & $\pm 20^{a b b}$ & 381 & $\pm 20^{\mathrm{a}}$ & 449 & $\pm 2 q^{a b}$ & 464 & \pm 22 & & \\
\hline & 0.5 & 522 & \pm 20 & 583 & \pm 25 & 476 & \pm 22 & 527 & \pm 21 & 523 & \pm 22 & \multirow[t]{2}{*}{0.006} & \multirow[t]{2}{*}{0.05} \\
\hline & 2.0 & 521 & \pm 29 & 656 & \pm 28 & 543 & \pm 36 & 580 & \pm 26 & 566 & \pm 23 & & \\
\hline
\end{tabular}

$\begin{array}{cccccccccc}\text { VO}_{2} \text { plac } & 107.5 \pm 6.4 & 98.3 \pm 7.6 & 76.1 \pm 4.6 & 97.5 \pm 5.4 & 82.6 \pm 5.7 & & \\ & 0.5 & 114.8 \pm 6.7 & 97.6 \pm 9.0 & 77.1 \pm 4.6 & 112.4 \pm 5.3 & 103.1 \pm 5.7 & 0.25 & 0.61 \\ & 2.0 & 104.3 \pm 7.9 & 92.5 \pm 7.6 & 83.7 \pm 6.8 & 112.7 \pm 5.5 & 91.7 \pm 9.0 & & \\ & & & & & & & & \\ \mathrm{HCO}_{3} \text { plac } & - & 24.9 \pm 0.4 & 22.5 \pm 0.4 & 25.7 \pm 0.4 & 30.4 \pm 4.1 & & \\ & 0.5 & - & 24.0 \pm 0.3 & 24.7 \pm 2.7 & 24.8 \pm 0.4 & 25.5 \pm 0.4 & 0.61 & 0.16 \\ & 2.0 & - & 23.8 \pm 0.4 & 22.7 \pm 0.5 & 24.7 \pm 0.4 & 25.3 \pm 0.4 & & \\ & & & & & & & & \\ \text { Lact. plac } & 1.2 \pm 0.1 & 0.9 \pm 0.1 \mathrm{1c} & 1.1 \pm 0.1 \mathrm{c} & 1.4 \pm 0.1 & 1.1 \pm 0.1 & 0.25 & 0.59 \\ & 0.5 & 1.4 \pm 0.2 & 0.9 \pm 0.1 & 1.0 \pm 0.1 & 1.8 \pm 0.2 & 1.3 \pm 0.2 & & \\ 2.0 & 1.1 \pm 0.1 & 1.2 \pm 0.1 & 1.6 \pm 0.1 & 1.8 \pm 0.2 & 1.2 \pm 0.1 & & \end{array}$

Values are shown as mean \pm standard errar. $\mathrm{DO}_{2}$, oxygen delivery $\left(\mathrm{m} / \mathrm{min} / \mathrm{m}^{2}\right)_{;} \mathrm{VO}_{2}$ oxygen consumption $\left(\mathrm{m} / \mathrm{min} / \mathrm{m}^{2}\right)_{\text {; }}$ $\mathrm{HCO}_{3}$ anterial bacarbonate; Lact, arterial lactate; Plac, placebo; 0.5 and 2.0 denote 0.5 and 2.0 dopexamine, respectively. Data were analysed using multiple-way ANOVA for all time-points, $p_{1}$ indicates $p$-values of repeated time-measures $p_{2}$ indicates the p-value of the interaction by multiple-way ANOVA between repeated measures $x$ dopexamine treatment.

${ }^{a} p<0.05$ comparing placebo versus $0.5 \mu \mathrm{g} / \mathrm{kg} / \mathrm{min}$ subgroups, ${ }^{\mathrm{b}} \mathrm{p}<0.05$ comparing placebo versus $2.0 \mu \mathrm{g} / \mathrm{kg} / \mathrm{min}$ subgroups, ${ }^{c} p<0.05$ comparing 0.5 and $2.0 \mu \mathrm{g} / \mathrm{kg} / \mathrm{min}$ subgroups.

least one time-point. These percentages were 45 and $49 \%$ for patients in the 0.5 and $2.0 \mu \mathrm{g} / \mathrm{kg} / \mathrm{min}$ dopexamine group, respectively.

In the low pHi group, the addition of dopexamine resulted in a significant increase in the post-operative $\mathrm{pHi}$, when compared to the placebo group $(p=0.04$ for timerelated influences and $p=0.008$ for time $x$ treatement related influences by multipleway ANOVA, see statistics). In addition, gastric pHi reached normal values in patients receiving dopexamine, while this was not the case in placebo treated patients (Figure 3). When after 24hrs dopexamine was stopped a significant decrease in gastric $\mathrm{pHi}$ from a mean of 7.34 to 7.30 was found when combining both treatment groups $(p=0.03)$.

The increase in gastric $\mathrm{pHi}$ associated with the use of dopexamine was not seen in the normal $\mathrm{pHi}$ group. Post-operatively, $\mathrm{pHi}$ remained similar in patients receiving dopexamine when compared to placebo (treatment-effect, $p=0.07$ ) (Figure 4). 
Table 3 Peri-operative changes in global hemodynamic perfusion during treatment with dopexamine in patients with a low pHi pre-operatively.

\begin{tabular}{|c|c|c|c|c|c|c|c|c|c|c|c|c|c|}
\hline \multicolumn{2}{|c|}{ Subgroups } & \multicolumn{2}{|c|}{ Pre-dopex } & \multicolumn{2}{|c|}{ Pre-op } & \multicolumn{2}{|c|}{ Post-op (PO) } & \multicolumn{2}{|c|}{$24 \mathrm{hPO}$} & \multicolumn{2}{|c|}{ 36h PO } & \multirow{2}{*}{$\begin{array}{c}p_{1} \\
0.007\end{array}$} & \multirow{2}{*}{$\begin{array}{c}p_{2} \\
0.25\end{array}$} \\
\hline $\mathrm{DO}_{2}$ & plac & 518 & \pm 19 & 458 & $\pm 23^{c}$ & 421 & \pm 28 & 546 & \pm 25 & 559 & \pm 26 & & \\
\hline & 0.5 & 518 & \pm 28 & 514 & \pm 30 & 499 & \pm 35 & 536 & \pm 31 & 536 & \pm 30 & & \\
\hline & 2.0 & 477 & \pm 34 & 590 & \pm 34 & 541 & \pm 37 & 534 & \pm 27 & 572 & \pm 37 & & \\
\hline \multirow[t]{3}{*}{$\mathrm{VO}_{2}$} & plac & 119.6 & \pm 7.9 & 89. & \pm 7.2 & 79.5 & $\pm 6.4^{c}$ & 121.0 & \pm 7.2 & 90.2 & \pm 8.5 & 0.006 & 0.99 \\
\hline & 0.5 & 118.7 & \pm 8.5 & 80. & \pm 4.8 & 86.3 & \pm 8.3 & 106.6 & \pm 6.6 & 105.8 & \pm 7.8 & & \\
\hline & 2.0 & 107.2 & \pm 8.2 & 92. & \pm 8.4 & 95.7 & \pm 6.8 & 105.7 & 6.0 & 98.2 & \pm 9.9 & & \\
\hline \multicolumn{2}{|c|}{$\mathrm{HCO}_{3}$ plac } & \multicolumn{2}{|c|}{. } & 29.5 & \pm 6.8 & \multicolumn{2}{|c|}{$20.9 \pm 0.6$} & \multicolumn{2}{|c|}{$22.4 \pm 0.7$} & 22.6 & $\pm 0.8^{c}$ & \multirow[t]{3}{*}{0.51} & \multirow[t]{3}{*}{0.52} \\
\hline & 0.5 & & . & 22. & \pm 0.5 & 21.4 & \pm 0.7 & 24.0 & \pm 0.6 & 24,7 & \pm 0.6 & & \\
\hline & 2.0 & & . & 32.1 & \pm 9.2 & 21.4 & \pm 0.4 & 24.4 & \pm 0.8 & 25.5 & \pm 0.7 & & \\
\hline \multirow[t]{3}{*}{ Lact. } & plac & \multicolumn{2}{|c|}{$1.0 \pm 0.1$} & 0.5 & $\pm 0.1^{b c}$ & \multicolumn{2}{|c|}{$1.2 \pm 0.1 \mathrm{bc}$} & 1.8 & \pm 0.2 & 1.1 & \pm 0.1 & \multirow[t]{2}{*}{0.19} & \multirow[t]{2}{*}{0.02} \\
\hline & 0.5 & \multicolumn{2}{|c|}{$0.9 \pm 0.1$} & 0.5 & \pm 0.1 & \multicolumn{2}{|c|}{0.1} & 1.4 & \pm 0.2 & \multicolumn{2}{|c|}{$1.2 \pm 0.2$} & & \\
\hline & 2.0 & \multicolumn{2}{|c|}{$1.2 \pm 0.1$} & 1.2 & \pm 0.1 & \multicolumn{2}{|c|}{$1.6 \pm 0.2$} & 1.6 & \pm 0.2 & \multicolumn{2}{|c|}{$1.2 \pm 0.2$} & & \\
\hline
\end{tabular}

Values are shown as mean \pm standard error. $\mathrm{DO}_{2}$ oxygen delivery $\left(\mathrm{ml} / \mathrm{min} / \mathrm{m}^{2}\right) ; \mathrm{VO}_{2}$ oxygen consumption $\left(\mathrm{m} / \mathrm{min} / \mathrm{m}^{2}\right)$; $\mathrm{HCO}_{3}$, arterial bacarbonater Lact, arterial lactate; Plac, placebo; 0.5 and 2.0 denote 0.5 and 2.0 dopexamine, respectively. Data were analysed using multiple-way ANOVA for all time-points. $p_{1}$ indicates $p$-values of repeated time-measures $p_{2}$ indicates the $p$-value of the interaction by multiple-way ANOVA between repeated measures $x$ dopexamine treatment. ${ }^{a} \mathrm{p}<0.05$ comparing placebo versus $0.5 \mu \mathrm{g} / \mathrm{kg} / \mathrm{min}$ subgroups, ${ }_{i} \mathrm{p}<0.05$ comparing 0.5 and $2.0 \mu \mathrm{g} / \mathrm{kg} / \mathrm{min}$ subgroups, $c_{p}<0.05$ comparing placebo versus $2.0 \mu \mathrm{g} / \mathrm{kg} / \mathrm{min}$ subgroups.

\section{Global oxygen transport}

Pre-operative haemodynamics prior to dopexamine infusion were similar between the two subgroups, with respect to the MAP, wedge-pressure, $\mathrm{DO}_{2}, \mathrm{VO}_{2}$, mixed venous oxygen saturation, actual bicarbonate, and lactate levels.

Global oxygen transport data for the normal $\mathrm{pHi}$ and low $\mathrm{pHi}$ group are shown in tables 2 and 3, respectively. In the normal pHi group the oxygen delivery was increased in the patients receiving dopexamine (treatment-effect, $p=0.05$ for $\mathrm{DO}_{2}$ ) (Table 2). In the low pHi group, the $\mathrm{DO}_{2}$ and $\mathrm{VO}_{2}$ were not altered by dopexamine (treatment-effect, $\mathrm{p}=0.3$ and treatment-effect, $\mathrm{p}=1.0$ for $\mathrm{DO}_{2}$ and $\mathrm{VO}_{2}$, respectively) (Table 3 ).

In the low $\mathrm{pHi}$ group dopexamine increased the arterial lactate (treatmenteffect $=0.02$ ), whereas the arterial lactate levels remained similar between the three treatment groups in the normal $\mathrm{pHi}$ group.

In order to detect differences in the systemic acid-base status as confounder 
of the gastric $\mathrm{pHi}$ measurement, the arterial actual bicarbonate levels were compared between the treatment subgroups in the low and normal pHi group. No significant changes in arterial bicarbonate levels were seen over time (timeeffect, $p=0.7$ and 0.5 ), nor did treatment with dopexamine alter the arterial bicarbonate levels (treatment-effect, $\mathrm{p}=0.2$ and 0.3 for normal and low $\mathrm{pHi}$ groups, respectively).

The total volume of crystalloids and colloids infused in the patients during the perioperative period was also equal in the low and normal pHi groups, as was use of other inotropes than dopexamine during the surgical procedure (data not shown).

\section{Mortality}

Logistic regression analysis, including $\mathrm{pHi}_{,} \mathrm{DO}_{2}$ and treatment as variables in the equation, showed that both $\mathrm{pHi}$ and global oxygen delivery were predictive for the occurrence of mortality for all patients combined. The measurement of $\mathrm{pHi}$ when obtained pre-operatively was the most important determinant of mortality ( $\mathrm{pHi}$ : $\mathrm{B}=-3.97, \mathrm{SE}=1.73, \mathrm{p}=0.02 ; \mathrm{DO}_{2}: \mathrm{B}=-0.003, \mathrm{SE}=0.002, \mathrm{p}=0.04$, Constant: $B=28.29, S E=12.75, p=0.03$ ).

Survival was not improved by adding dopexamine in the subgroup with a low pHi. Day 28 mortality in patients with a low pHi receiving placebo treatment was $20.5 \%(8 / 39)$, whereas mortality was $10.8 \%(4 / 37)$ and $18.9 \%(7 / 37)$ in patients with 0.5 and $2.0 \mu \mathrm{g} / \mathrm{kg} / \mathrm{min}$, respectively $(p=0.5)$. Mortality in the different subgroups in patients with a normal pHi was $3.6 \%(2 / 56), 1.6 \%(1 / 61)$, and $1.9 \%(1 / 53)$ for the placebo, 0.5 and $2.0 \mu \mathrm{g} / \mathrm{kg} / \mathrm{min}$ treated patients, respectively $(p=0.1)$. The adjusted odds ratio for mortality of the low $\mathrm{pHi}$ group versus normal $\mathrm{pHi}$ group is 8.5 , with an unadjusted odds ratio of 7.0 for the placebo treated patients when comparing low and normal pHi groups and 7.3 and 12.1 for the 0.5 and $2.0 \mu \mathrm{g} / \mathrm{kg} / \mathrm{min}$ treated patients, respectively (mean: 8.8 ).

\section{Morbidity}

The MOF-score during the 27 day follow-up period in the low pHi placebo treated patients was higher when compared with the normal pHi group (treatment-effect, $p=0.001$ ). The mean MOF-score after $24 \mathrm{~h}$ was 2.7 (SD 2.6) in the low $\mathrm{pHi}$ group and 1.6 (SD 1.4) in the normal pHi group $(p=0.02)$. Logistic regression analysis showed that in predicting the occurrence of multiple organ failure, the measurment of pre-operative gastric $\mathrm{pHi}$ was the most important predictor $(\mathrm{pHi}: \mathrm{B}=-4.2, \mathrm{SE}=0.55$, $p<0.0001 ; D_{2}: B=-0.0009, S E=0.0004, p=0.03 ;$ Constant: $B=29.5, S E=4.06$ ) In the low $\mathrm{pHi}$ group the MOF-score in patients with dopexamine was significantly lower during the follow-up period of 27 days compared to patients treated with placebo (time-effect, $p=1.0$ and treatment-effect, $p=0.04$ ). Additionally in the low pHi group, the number of patients with multiple organ failure was significantly higher in the placebo versus dopexamine treated patients (12/39 vs. $3 / 37$ and $2 /$ 
37 for placebo, 0.5 , and $2.0 \mu \mathrm{g} / \mathrm{kg} / \mathrm{min}$, respectively: $\mathrm{p}=0.003$ ). The effect of dopexamine was mainly seen in the cardiovascular, pulmonary, haemotological, and renal components of the MOF score:

MOF-scores in the normal pHi group did not change during treatment with dopexamine (treatment-effect, $p=0.26$ ). The number of patients with multiple organ failure was also similar $(3 / 58,6 / 81$, and $10 / 53$ for placebo, 0.5 , and $2.0 \mathrm{gg} /$ $\mathrm{kg} / \mathrm{min}$, respectively: $\mathrm{p}=0.07$ ).

\section{Discussion}

Reports in the literature concerning the use of supranormal values of global $\mathrm{DO}_{2}$ as goals for haemodynamic management have produced varying results. $A$ tentative conclusion can be made that trauma and high-risk peri-operative patients may benefit from such an approach, whereas septic ICU patients, in general, do not $(5-9,27)$. Recent studies indicated that not only global perfusion determines outcome $(11,28)$. Within the ICU, there is current interest in focussing on regional, rather than global, perfusion parameters. This study represents an evaluation of the relationship between regional and global perfusion in a group of high-risk surgical patients undergoing haemodynamic optimisation, with or without the addition of dopexamine.

In this analysis, a low pHi pre-operatively was associated with significantly higher mortality and morbidity compared to a normal $\mathrm{pHi}$. The relation between decreased $\mathrm{pHi}$ level and increased frequency of postoperative death and complications has been found previously $(10,21,22)$, but to our knowledge this is the first study which found this association when the gastric $\mathrm{pHi}$ was determined prior to the surgical procedure. Apparently, high-risk surgical patients have signs of splanchnic intramucosal acidosis prior to the surgical intervention. The measurement of intramucosal $\mathrm{PCO}_{2}$, from which the pHi is calculated, gives an indirect meāsurement of the adequacy of the mucosal or splanchnic blood flow. The $\mathrm{pHi}$ value represents a composite value which reflects several factors. Besides mucosal perfusion, systemic acid-base status, and changes in metabolism influence the final $\mathrm{pHi}$ measurement. It is likely that changes in perfusion or volume status contribute to the pre-operative intramucosal acidosis. Patients undergoing elective major abdominal surgery are starved for at least $10 \mathrm{~h}$ to reduce the risk of aspiration. Moreover, the diseases for which the patients are operated upon often caused weight loss, nausea or vomiting. All these factors can contribute to an increased risk of mucosal hypoperfusion and acidosis in the patient prior to surgery. In addition, previous data indicated that an intramucosal acidosis was associated with decreased gastric perfusion (29). Unfortunately, we did not record more information on the systemic acid-base status to indicated that the changes in $\mathrm{pHi}$ were based on differences in the regional $\mathrm{PCO}_{2}$ and not the arterial $\mathrm{pH}$, as indicated by the Henderson-Hasselbach equation. However, the lack of differences in the actual bicarbonate levels does suggest this. 
The cult-off point of a pHi of 7.35 or lower as marker for the presence of intramucosal acidosis has not been validated extensively. However, both in this study and in previous studies this was able to identify a high-risk surgical population (21-23,30). The cut-off point in this study was based upon a ROC-curve analysis. The area under the $\mathrm{ROC}$ curve of 0.79 indicated good predictive power in the individual patient. In this study the cut-off point of 7.35 showed a clear distinctive low mortality $(<3 \%$ ) in patients with a pHi above 7.35 and a markedly higher mortality $(>16 \%$ in patients with a pHi below 7.35 . Moreover, this study also indicated that the low pHil value was associated with an increased risk on MOF. Both mortality and the development of MOF were best predicted using the measurement of gastric $\mathrm{pHi}$, when assessed using a logistic regression analysis. The measurement of the gastric intramucosal pHi, therefore, seems to be an adequate predictor of mortality in high-risk surgical patients when measured prior to surgery. Although the patients in this study were already receiving dopexamine or placebo at the time of the first $\mathrm{pHi}$ measurement, the pre-operative $\mathrm{pHi}$ values were not different between placebo- and dopexamine-treated patients. However, the changes in $\mathrm{pHi}$ were strikingly different in the various treatment schedules in the post-operative period. In the low pHi group, both dopexamine treated groups showed a significant increase in $\mathrm{pHi}$ post-operatively when compared with the placebo group. Moreover, the decrease in pHi after cessation of the dopexamine infusion may indicate a rebound phenomenon, although an exact explanation for this finding is difficult to give.

Although many studies have been published before using dopexamine, only two other studies investigated the effects of dopexamine on gastric pHi during major abdominal surgery. Müller et al. showed a decreased tissue $\mathrm{PCO}_{2}$ in the small intestine during low-dose dopexamine $(0.5 \mu \mathrm{g} / \mathrm{kg} / \mathrm{min})$ infusion, although the gastric pHi did not change significantly (31). This latter observation may be related to the relatively short intra-operative infusion of dopexamine during 60 minutes. The effects of dopexamine in our study were found after $6 \mathrm{hrs}$. The study by Byers et $\mathrm{al}_{\text {, }}$ including 30 patients out of the database of the main dopexamine study, displayed no differences in gastric pHi, although a significant decrease in the inflammatory response in the gastric mucosa was found during dopexamine infusion (32). Two other studies in patients undergoing major abdominal surgery using dopexamine by Boyd et al. and Wilson et al. did not include measurements of gastric intramucosal pHi $(6,15)$.

The other studies using dopexamine included patients with severe septic shock yielding varying results of the effects if dopexamine $(33,34)$. Gastric pHi decreased during dopexamine infusion in patients with septic shock, despite an increase in total splachnic blood flow (33). The causes for this dissociation between total splanchnic blood flow and gastric pHi are multifactorial and may be related to the presence of heterogenous or inadequate blood flow within the hepatosplanchnic area during sepsis or may be related to the Haldane effect (35). It is also possible that the concommittant use of other vasoactive medications and sepsis-related changes in vasomotor autoregulation may contribute to these findings $(27,36)$.

After cadiac surgery dopexamine improved gastric pHi in one study and decreased 
it, despite an increase in total splanchnic blood flow, in another. It is possible that the effects of dopexamine can also be modified by the type of surgery $(37-40)$. In contrast to the low pHi group, in the normal pHi group no effect of the dopexamine treatment could be demonstrated, with mean $\mathrm{pH}$ Hi values remaining higher than 7.35. Both groups received fluids for correction of (global) hypowolaemia. We hypothesised that the low pHi group represent patients who were hypovolaemic prior to fluid loading, but in whom correction of systemic hypovolaemia did not result in restoration of splanchnic flow. Edouardo showed that transient normotensive hypovolaemia induces a splanchnic vasoconstriction that is sustained after normalising blood (41). The further interpretation of the data presented is made difficult by the fact that the protocol did not include pHi measurements prior to fluid loading and the start of dopexamine treatment.

The group in which dopexamine appeared to show a beneficial effect on outcome was in the low pHi group. The mean pHi values in the low pHi group did not return to normal levels in the placebo-treated patients, whereas after $6-12 \mathrm{~h}$ a normalisation was achieved in the dopexamine treated patients. In these patients MOF-scores and the occurrence of MOF were significantly lower compared to the placebo-treated patients, although mortality was not altered by dopexamine.

The change in $\mathrm{pHil}$ and MOF-score was not associated with a change in global $\mathrm{DO}_{2}$, a finding again supported by previous studies. In the study by Bach et al. dopexamine also improved splanchnic perfusion without a concomittant rise in $\mathrm{DO}_{2}$ (38). In a study by Ivatury et al. patients following trauma were actively resuscitated to either a $\mathrm{DO}_{2}$ of $>600 \mathrm{ml} / \mathrm{kg} / \mathrm{m}^{2}$ or a pHi of $>7.3(27)$. In this study improvement of $\mathrm{DO}_{2}$ without improved splanchnic perfusion was associated with a high mortality.

In the normal $\mathrm{pHi}$ group, in which dopexamine had no beneficial effect on $\mathrm{pHi}$, there was also no difference in MOF-scores between the dopexamine and placebo treated patients.

In conclusion, in this large study population of patients undergoing high-risk surgical procedures, pre-operative measurement of gastric mucosal pHi is a good predictor of postoperative morbidity and mortality. The peri-operative response of $\mathrm{pHi}$ to dopexamine seemed to be dependent on pre-operative gastric $\mathrm{pHi}$. In the low pHi group, an association between the effect of dopexamine on $\mathrm{pHi}$ and the effect on multiple organ failure was observed, independently of changes in global $\mathrm{DO}_{2}$. In light of the results of the published studies sofar and the retrospective nature of this analysis, routine treatment of a low pre-operative gastric pHi by dopexamine cannot be recommended before the beneficial effects of such a treatment on gastric oxygenation have been demonstrated in a prospective, randomised clinical trial. 


\section{REFERENCES}

1. Knaus WA, Oraper EA, Wagner DP, Zimmerman JE. Prognosis in acute organ-system failure. Ann Surg 1985;202: 685-693.

2. Moore FA, Haenel IB, Moore EE, Whitehill TA. Incommensurate oxygen consumption in response to maximal oxygen availability predicts postinjury multiple organ failure. I Trauma 1992,33: 58-65.

3. Shoemaker WC, Appel PL, Kram HB, Waxman MD, Lee T-S. Prospective trial of supranormal values of survivors as therapeutic goals in high-risk surgical patients. Chest 1988:94:1176-1186.

4. Connett $\mathrm{RJ}$, Honig CR, Gayeski TE), Brooks GA. Defining hypoxia: a systems view of $\mathrm{VO}_{2}$, glycolysis, energetics, and intracellular $\mathrm{PO}_{2}$. I Appl Physiol 1990;68:833-842.

5. Berlauk JF, Abrams JH, Gilmour II, $O^{\prime}$ Connor SR, Knighton DR, Cerra FB. Preoperative optimization of cardiovascular hemodynamics improves outcome in peripheral vascular surgery. A prospective, randomized clinical trial. Ann Surg 1991;214: 289-297.

6. Boyd $\mathrm{O}$, Grounds RM, Bennett ED A randomized clinical trial of the effect of deliberate perioperative increase of oxygen delivery on mortality in high-risk surgical patients. IAMA 1993;270: 2699-2707.

7. Bishop $M H$, Shoemaker WC, Appel $\mathrm{PL}_{\text {, Meade }}$, Ordog Gl, Wasserberger I, Wo C-f, Rimle DA, Kram HB, Umali R, Kennedy F, Shuleshko I, Stephen CM, Shori SK, Thadepalli HD. Prospective, randomized trial of survivor values of cardiac index, oxygen delivery, and oxygen consumption as resuscitation endpoints in severe trauma. I Trauma 1995;38:780-787.

B. Fleming $A$, Bishop $M$, Shoemaker W, Appel P, Sufficool W, Kuvhenguwha A, Kennedy $F$, Wo C.J. Prospective trial of supranormal values as goals of resuscitation in severe trauma. Arch Surg 1992;127:1175-1181.

9. Hayes MA, Timmins AC, Yau EHS, Palazzo M, Watson D, Hinds CJ. Oxygen transport patterns in patients with sepsis syndrome or septic shock: influence of treatment and relationship to outcome. Crit Care Med 1997;25: 926-936.

10. Fiddian Green RG. Associations between intramucosal acidosis in the gut and organ failure. Crit Care Med 1993;21:S103-5107.

11. Mythen MG, Webb AR.Perioperative plasma wolume expansion reduces the incidence of gut mucosal hypoperfusion during cardiac surgery. Arch Surg 1995;130:423-429.

12. Cain SM, Curtis SE. Systemic and regional oxygen uptake and delivery and lactate flux in endotoxic dogs infused with dopexamine. Crit Care Med 1991; 19:1552-1560.

13. Bredle DL, Cain SM. Systemic and muscle oxygen uptake/delivery after dopexamine infusion in endotoxic dogs. Crit Care Med 1991;19:198-204.

14. Smithies $M N$, Yee $T H$, Jackson $L$, Beale $R$, Bihari D. Protecting the gut and the liver in the critically ill: effects of dopexamine. Crit Care Med 1994;22:789-795.

15. abWilson J, Woods 1, Fawcett ]. Whall R, Dibb W, Morris C, McManus E. Reducing the risk of major elective surgery: randomised controlled trial of preoperative optimisation of oxygen delivery، BMI 1999;318:1099-1103.

16. abUusaro A, Ruokonen E, Takala J. Splanchnic oxygenation transport after cardiac surgery: evidence for inadequate tissue perfusion after stabilisation of hemodynamics. Intensive Care Med 1995;614:1-8.

17. Cohen IL, Lumb PD. Monitoring tissue oxygen perfusion: global or regional. Intens Crit Care Digest 1991;10:56-59. 


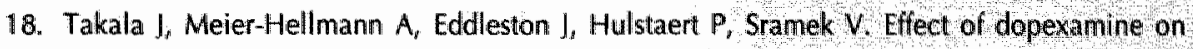
outcome after major abdominal surgery. A prospective, randomized, controlled multicenter study. Crit Care Med 2000 lin press].

19. Bland RD, Shoemaker WC, Shabot MM. Physiologic monitoring goals for the critically ill patient. Surg Gynecol Obstet 1978147:833-841.

20. Goris RI, te Boekhorst TP, Nuytinck IK, Gimbrere JS Multiple-organ failure Generalized autodestructive inflammation? Arch Surg 1985;120:1109-1115.

21. Kirton $O C_{,}$Windsor J, Wedderburn $R$, Hudson-Civetta I, Shatz DV, Mataragas NR, Civetta:M, Failure of splanchnic resuscitation in the acutely injured trauma patient correlates with multiple organ system failure and length of stay in the ICU. Chest 1998;11311064-1069.

22. Maynard ND, Taylor PR, Mason RC, Bihari DJ. Gastric intramucosal pH predicts outcome after surgery for ruptured abdominal aortic aneurysm. Eur J Vasc Endovasc Surg 1996;11 201-206.

23. Gutierrez G, Palizas F, Doglio G, Wainsztein N, Gallesio A, Pacin I, Dubin A, Schiavi E, lorge M, Pusajo I. Gastric intramucosal $\mathrm{pH}$ as a therapeutic index of tissue oxygenation in citically ill patients. Lancet 1992;339:195-199.

24. Hanley $\rfloor A$, MCNeil $B$ ). The meaning and use of the area under the a recelver operating: characteristic (ROC) curve. Radiology 1982;143:29-36.

25. Hanley JA, McNeil BJ. A method for comparing the areas under receiver operating characteristic curves derived from the same cases. Radiology 1983;148:839-843.

26. Poeze M, Ramsay $G$, Greve JWM, Singer $M$. Prediction of postoperative cardiac surgical morbidity and organ failure within 4 hours of ICU admission using esophageal Doppler ultrasonography. Crit Care Med 1999;127:1288-1294.

27. Poeze $M$, Greve M, Ramsay M. Oxygen delivery and septic shock [letter]. Chest 1999;116:1145.

28. Ivatury RR, Simon RI, Islam SI, Fueg A, Rohman M, Stahl WM. A prospective randomized study of end points of resuscitation after major trauma: global oxygen transport indices versus organ-specific gastric mucosal pH. I Am Coll Surg 1996;183:145-154.

29. Elizalde II, Hernandez C, Llach J, Manton C, Bordas JM, Pique IM, Torres A. Gastric intramucosal acidosis in mechanically ventilated patients: role of mucosal blood flow. Crit Care Med $1998 ; 26: 827-832$.

30. Chang $M C$, Meredith JW. Cardiac preload, splanchnic perfusion, and their relationship during resuscitation in trauma patients. I Trauma 1997;42:577-582.

31. Müller M, Boldt J, Schincller $E$, Sticher J, Kelm $C$, Roth $S$, Hempelmann G. Eiffects of low-dose dopexamine on splanchnic oxygenation dduring major albdominal surgery. Crit Care Med 1999;27:2389-2393.

32. Byers RJ, Eddleston IM, Pearson RC, Bigley G, McMahon RFT. Dopexamine reduces the incidence of acute inflammation in the gut mucosa following abdominal surgery in high risk patients. Crit Care Med 1999;27:1787-1793.

33. Meier-Hellmann A, Bredle DL, Spechit $M_{r}$ Hannemann L, Reinhart K. Dopexamine increases splanchnic blood flow but decreases gastric mucosal $\mathrm{pH}$ in severe septic patients treated with dobutamine. Crit Care Med 1999;27:2166-2171.

34. Temmesfelld-Wollbrück B, Szalay $A$, Mayer $K$, Olschewski $H$, Seeger $W$, Crimminger $F$. Abnormalities of gastric mucosal oxygenation in septic shock: partial responsiveness to dopexamine. Am J Resp Crit Care Med 1998;157:1586-1592.

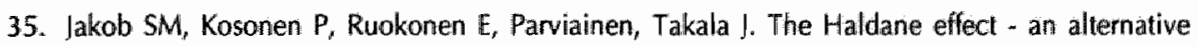
explanation for increasing gastric mucosal $\mathrm{PCO}_{2}$ gradients? Br J Amaesth 1999;83:740-746. 
36. Poeze M, Greve JWM, Ramsay $G$. Coal-oriented haemodynamic therapy: a plea for a closer llook at using peri-operative oxygen transport optimisation. Intensive Care Med 2000 lin press].

37. Uusaro $\mathrm{A}$, Ruokonen $\mathrm{E}$, Takala f. Gastric mucosal $\mathrm{pH}$ does not reflect changes in splanchnic blood flow after cardiac surgery. Br) Anaesth 1995;74:149-154.

38. Bach $F$, Sillomon $M$, Grundman $U$, Sturner $I$, graeter $T$, Larsen $R$. Effects of dopexamine on splanchnic perfusion in cardiac surgery. Anaesthesist 1999;48: 713-717.

39. Johnston WE, Zwischenberger $1 B$. Improving splanchnic perfusion during cardiopulmonary bypass [editorial]. Anesthesiology 2000,92:305-307.

40. Hellebrekers LI, Liard IF, Woodson LC, Lin CY, Deyo DJ, Uchida T, Jahnston WE. Regional autoregulatory responses during infusion of vasoconstrictor agents in conscious dogs. Am J Physiol 1990,259:H1270-H1277.

41. Edouard AR, Dergremont A-C, Duranteau J, Pussard E, Berdeaux A, Samii K. Heterogeneous regional vascular responses to simulated transient hypovolemia in man. Intensive.Care Med $1994 ; 20: 414,420$. 


\section{CHAPTER 11}

Increased hepatosplanchnic inflammation precedes the development of organ

dysfunction after elective high-risk surgery 


\section{ABSTRACT}

Objective. This study investigated the relationship of the hepatosplanchnic production and uptake of inflammatory mediators, hepatosplanchnic perfusion, and outcome during major abdominal surgery to evaluate the hypothesis that regional production of inflammatory mediators precedes the development of hepatic dysfunction.

Design: Retrospective analysis of data and blood samples collected during a randomised controlled clinical trial:

Setting, 24 -bed university-afilliated intensive care unit.

Patients. High-risk surgical patients undergoing major abdominal surgery.

Interventions: none.

Measurements: Patients were divided into a subgroup that developed hepatic dysfunction $(\mathrm{HD}+1)$ postoperatively and a subgroup without hepatic dysfunction (HD-). Hepatic vein and arterial plasma levels of IL-6, IL-8, s-E-selectin, s-ICAM-1, and the TNF-receptors 55 and 75 were measured, and the flux was calculated by multiplying the difference in hepatic vein minus arterial levels of the mediators by the hepatosplanchnic flow. Systemic (thermodilution) and total hepatosplanchnic blood flow (using indocyanine green (ICG)-dilution method) and gastric intramucosal $\mathrm{pH}$ (pHi) were assessed pre-operatively, 4, 24 and $36 \mathrm{hrs}$ post-operatively.

Results. Of a total of 26 patients, six patients developed hepatic dysfunction after their abdominal surgery (mean 6 days postoperatively). The number of sepsisrelated deaths and post-operative days on the ventilator were significantly higher in this group. A higher production of IL-8, TNF-receptor-75 and 55 in the hepatosplanchnic area in the $\mathrm{HD}+$ subgroups was found, which preceeded the development of organ dysfunction ( $p=0.04, p=0.02$, and $p=0.02$, respectively). Moreover, the uptake of s-ICAM-1 was significantly increased in this subgroup. Furthermore, total hepatosplanchnic blood flow was significantly higher and $\mathrm{pHi}$ was significantly lower in the $\mathrm{HD}+$ group, whereas global haemodynamic data were similar in the two subgroups.

Conclusion: The development of post-operative organ dysfunction is preceded by an increased regional inflammatory response, indicated by an increased soluble TNF-receptor shedding and IL-8 production from the hepatosplanchnic area together with an increased uptake of s-ICAM-1. Moroever, an increased total hepatosplanchnic blood flow with intramucosal acidosis was associated with this regional inflammatory response. 


\section{INTRODUCTION}

An increased inflammatory response may contribute to the development of organ failure in critically ill patients (1). After surgery, increased systemic levels of acute phase cytokines and mediators of neutrophil adhesion and activation were associated with the development of multiple organ failure (MOF) both in experimental and clinical studies (2-6). This increase in systemic levels is thought to result from an increased and exagerated local inflammatory response in the tissues (7). Animal studies suggest that the hepatosplanchnic area may be an important site of production of the inflammatory mediators in response to a surgical trauma (8), in patients with established MOF regional production of inflammatory mediators has been observed (9). Alternatively, a decreased clearance from the circulation by the liver may result in increased systemic levels of inflammatory mediators.

Alteration in hepatosplanchnic perfusion is considered to be an important factor in the development of post-operative organ failure. Indeed, signs of inadequate perfusion have been observed in patients with an increased risk of post-operative organ failure and may contribute to postoperative organ dysfunction both directly and by enhancing the inflammatory response $(10,11)$. Although the relationship between the regional perfusion disturbances and the inflammatory response is incompletely understood, inadequate splanchnic tissue perfusion has been associated with increased systemic levels of inflammatory mediators (12).

Therefore, we hypothesized that the blood levels of cytokines and the hepatosplanchnic kinetics of inflammatory mediators during and in the early stages after surgery may be different in patients developing subsequent organ dysfunction compared with patients with an uncomplicated course. To test this hypothesis, we evaluated whether there is a relationship between the activation of the inflammatory response in the hepatosplanchnic area and the development of hepatic dysfunction during the following post-operative period. We also evaluated whether changes in the regional blood flow and oxygen transport response were related to the changes in hepatosplanchnic kinetics of the inflammatory mediators.

\section{PATIENTS AND METHODS}

\section{Patient selection}

Patients were all entered in a multicenter trial studying the effects of haemodynamic optimisation with fluids and dopexamine (13). In 1 center 26 patients received a hepatic venous catheter in addition to the study protocol and these patients formed the study group. Institutional ethics committee approval was obtained and patients or close relatives gave informed consent for enrolment in 
the study. Patients undergoing non-vascular, abdominal surgery and fulfilling one or more of the high-risk criteria of Bland et al. (14) were included in the study population. Patients were enrolled if the operation was expected to last for more than 1.5hrs. A total of 26 patients were included.

\section{Peri-operative treatment and assessment}

On admission to the intensive care unit (ICU) patients received a pulmonary artery occlusion catheter and an intra-arterial catheter. Cardiac output was measured by thermodilution from the average of triplicate injections of $5 \mathrm{ml}$ of cold $0.9 \% \mathrm{NaCl}$. Arterial blood gas and arterial and mixed venous oxygen saturation levels were measured simultaneously with the cardiac output measurements.

During pre-operative stabilisation fluids, blood and/or oxygen were given if patients had a wedge pressure less than $10 \mathrm{mmHg}$, a cardiac index less than $2.5 \mathrm{l} / \mathrm{min} / \mathrm{m}^{2}$, mean arterial pressure less than $70 \mathrm{mmHg}$, a haemoglobin concentration of less than $10 \mathrm{~g} / \mathrm{dl}$ or a $\mathrm{SaO}_{2}$ less than $94 \%$. Fluids were given throughout the peri-operative period as required to maintain the above mentioned goals. At any time a vasopressor agent or inotropes could be used when fluids alone were not sufficient. In the randomised trial from which these patients were selected, dopexamine was added at a fixed dose of either $0.5 \mu \mathrm{g} / \mathrm{kg} / \mathrm{min}$ or $2.0 \mu \mathrm{g} / \mathrm{kg} / \mathrm{min}$ (13). At the start of surgery, directly after surgery, and 2, 6, 12, 24 and 36hrs after surgery the following parameters were recorded: systolic and diastolic arterial and pulmonary artery pressures, heart rate, right arterial pressure, arterial and mixed venous oxygen saturation, core temperature, pulmonary wedge pressure, mean cardiac output, haemoglobin, lactate and creatinine concentrations. Furthermore, from the start of surgery until 36hrs postoperatively, gastric intramucosal $\mathrm{pH}(\mathrm{pHi})$ was determined at the above mentioned intervals using a gastrointestinal tonometer as described previously (15). Renal function was assessed by creatinine clearance and urinary flow rate from $0-24 \mathrm{hrs}$ and $24-36 \mathrm{hrs}$ post-operatively: Moreover, total hepatosplanchnic blood flow was measured by indocyanine green (ICG) dye dilution, measured at stabilization, 4, 24, and 36hrs postoperatively (16). For this purpose catheters were placed into the femoral artery and the right hepatic vein. ICG was infused with a priming dose of $12 \mathrm{mg}$ followed by a constant infusion rate of $1.1 \mathrm{mg} / \mathrm{min}$ for 30 mins. At 20, 25, and 30 mins after the first dose, blood samples were drawn from the femoral artery and the hepatic vein for measurement of ICG concentrations. The first $\mathrm{pHi}$ measurement was performed after the induction of anaesthesia.

\section{Patient follow-up}

Post-operatively, patients were readmitted to the ICU. The same goals set preoperatively were used postoperatively in order to optimise the haemodynamic condition of the patient. The Acute Physiologic And Chronic Health Evaluation (APACHE) II- and MOF-scores (modified from Goris et al.(17)) were calculated 
from data obtained after $24 \mathrm{~h}$-stay on the ICU. From 30hrs after surgery onwards, patients could be discharged from the ICU whenever their condition was thought suitable. After discharge from the ICU, patients were monitored for 27 days for morbidity and mortality. At day 2 to 6 , day 13,20 and 27 organ failure was assessed by the MOF-score and clinical data.

Hepatic dysfunction ( $\mathrm{HD}+$ ) using the MOF-score as defined by Goris et al. was defined as a bilirubin level $>34 \mu \mathrm{mol} / \mathrm{l}$ and SGOT (AST) $>70 \mathrm{U} / \mathrm{l}$ for at least three days $(9,17)$. Treatment with dopexamine or placebo was equally distributed among patients in the HD+ and HD- group (placebo 3 vs. 8; 0.52 vs. $8 ; 2.0 \mu \mathrm{g} / \mathrm{kg} / \mathrm{min} 1$ vs. 4).

\section{Cytokine analysis}

Plasma samples for cytokine and adhesion molecule measurement were obtained from the hepatic vein and radial artery before surgery and 4,24 and 36hrs after surgery in tubes containing EDTA, which were immediately put on ice. Plasma was prepared at $4{ }^{\circ} \mathrm{C}$ by centrifugation at $2,200 \mathrm{~g}$ for 5 mins. Samples were stored at $70{ }^{\circ} \mathrm{C}$ until analysed.

Levels of inflammatory mediators were determined as previously described (18-21). In short, interleukin-6 was caught by monoclonal antibody (Mab) 5E1 (developed in our laboratory) and detected by biotin-labelled polyclonal rabbit anti-human IL-6 IgG. The standard used was recombinant human (rh)lL-6 (provided by Dr. Sebald, Wuerzberg, Germany). IL- 8 was detected by biotin labelled rabbit antihuman IL-8 after capture by the Mab HM.7. In the IL-8 ELISA rhIL-8 (provided by Dr. Lindley, Sandoz, Vienna, Austria) was used as standard. Soluble ICAM-1 and soluble E-selectin were captured using Mabs HM.2 (anti-sICAM-1), and ENA-1 (anti-E-selectin) and detected with biotinylated Mab HM.1 and biotin-labeled Mab ENA2, respectively $(20,22)$ (developed in our laboratory). The standards used were rh-ICAM-1 and rh-sE-selectin, respectively.

For measurement of sTNF-R55 and sTNF-R75, mAbs MR1-1 and MR2-2 were used for coating, respectively (provided by Dr. M. Brockhaus, F. Hoffmann-La Roche, Basel, Switzerland). Specific biotin-labeled polyclonal rabbit anti s-TNF-R IgG were used as detector reagents (23). The standards used were rh s-TNF-R55 and s-TNF-R75. Detection limits of IL-6, IL-8, ICAM-, E-selectin, R75 and R55 were $10 \mathrm{pg} / \mathrm{ml}, 10,400,1(\mathrm{ng} / \mathrm{ml}), 100$, and $100 \mathrm{pg} / \mathrm{ml}$, respectively.

The fluxes of the cytokines over the hepatosplanchnic area were calculated by multiplying the difference in hepatic vein minus arterial levels of the mediators by the hepatosplanchnic flow.

\section{Statistics}

Data from patient characteristics comparing both subgroups are presented as mean (SEM) or as percentages and were assessed using Student t-test and chisquare test, respectively. 
The absolute cytokine levels and fluxes are presented as mean with standard error. The hepatosplanchnic fluxes were calculated by multiplying the difference in hepatic vein minus arterial blood levels of the inflammatory mediators by the ICGmeasured flow. The cytakine levels and fluxes and haemodynamic data between the subgroups were compared using multiple-way ANOVA for correction of timerelated influences with the dopexamine treatment as covariate. Error values of the multiple way ANOVA are reported as $\mathrm{p1}$, to indicate the significance of the within-subject (time-related) analysis and $\mathrm{p} 2$ to indicate the significance of the interaction between repeated measures $x$ presence of hepatic organ dysfunction (between-subject analysis). The treatment with dopexamine was used as covariate in this analysis. In case this analysis was significant, individual time-points were analysed using the Mann-Whitney $U$ test. The cytokine fluxes were compared to zero (i.e. measurement of significant flux over the hepatosplanchnic area) using the one-sample Komolgorov-Smirnov test. A p-value below 0.05 was considered statistically significant. Correlations were calculated with Pearson coefficients between gastric $\mathrm{pHi}$ and hepatic vein minus $(-)$ arterial cytokine levels for both patients with uncomplicated and complicated surgical procedures.

\section{RESULTS}

\section{Patients}

In total, 26 high-risk surgical patients were included into the analysis. All patients underwent elective surgical procedures, and none of the patients were clinically septic during the study period. Of these, 22 patients underwent elective highrisk surgery for a gastro-intestinal or pancreatic carcinoma. Six patients of the 22 patients with a carcinoma were found to have metastases at the time of surgery. Of these six patients 2 patients were in the group with hepatic dysfunction. One patient was operated upon because of diverticulitis coli, one had ulcerative colitis, and one patient was operated upon for an enterocutaneous fistula. One patient was operated upon because of an acute appendicitis, but this patient was haemodynamically stable and did not have or develop multiple organ or hepatic dysfunction.

Mean duration of the surgical procedures was $197 \pm 105$ minutes. APACHE-score at $24 \mathrm{hrs}$ post-operatively for all patients was $10.1 \pm 5.0$. Mean MOF-score after 24 hrs was $1.2 \pm 1.3$ for all patients.

Patients were divided into 2 groups with $(\mathrm{HD}+)$ or without (HD-) post-operative hepatic dysfunction. Pre-operatively, liver function was within normal limits in all patients. After a mean post-operative period of 6 days, thepatic dysfunction developed in six patients (Table 1). Patients in both subgroups were comparable with 
Table 1 Patient demographics in patients who did and did not develop hepatic organ dysfunction

\begin{tabular}{lll}
\hline Variables & $\begin{array}{l}\text { Hepatic }(\mathrm{HO}+) \\
\text { dysfunction }\end{array}$ & $\begin{array}{l}\text { No hepatic (HD-) } \\
\text { dysfunction }\end{array}$ \\
\hline
\end{tabular}

Number

6

20

\begin{tabular}{|c|c|c|c|c|c|}
\hline Age (years) & 64.1 & \pm 159 & 61.5 & \pm 9.1 & 0.3 \\
\hline Cender (M/F) & $2 / 4$ & & $8 / 12$ & & 0.8 \\
\hline Weight (kg) & 68.0 & \pm 12.2 & 79.5 & \pm 22.1 & 0.1 \\
\hline \multicolumn{6}{|l|}{ Surgical procedure } \\
\hline Upper Gl procedure (\%) & 33 & & 25 & & \\
\hline Lower GI procedure (\%) & 67 & & 75 & & 0.6 \\
\hline Number of patients with metastases & 3 & & 3 & & 0.1 \\
\hline Time of surgery (min) & 286 & \pm 150 & 170 & \pm 72 & 0.6 \\
\hline \multicolumn{6}{|l|}{ Blood loss (1) } \\
\hline during surgery & 1.8 & \pm 2.5 & 1.2 & \pm 0.8 & 0.5 \\
\hline post-operatively & 0.8 & \pm 0.4 & 0.5 & \pm 0.4 & 0.6 \\
\hline APACHE-\|l score (at 24 hours) & 10.7 & \pm 6.5 & 10.0 & \pm 4.8 & 0.4 \\
\hline MOF-score (at 24 hours) & 3.0 & \pm 1.0 & 0.7 & \pm 0.8 & 0.001 \\
\hline
\end{tabular}

Hepatic function 24 hours post-operatively

\begin{tabular}{|c|c|c|c|c|c|}
\hline AST $(\|\cup\|)$ & $\$ 39.1$ & \pm 467.9 & 25.4 & \pm 19.3 & $0.0 \%$ \\
\hline ALT $(\| \cup /)$ & 140.0 & \pm 319.7 & 22.7 & $\pm 21,0$ & 0,001 \\
\hline Bilirubin (mmol/l) & 34.0 & \pm 45.7 & 11.1 & \pm 7.2 & 0.001 \\
\hline \multicolumn{6}{|c|}{ Renal function 24 hours post-operatively } \\
\hline Urinary flow rate $(\mathrm{m} / / \mathrm{kg}$ hour) & 3.0 & \pm 0.7 & 2.7 & \pm 1.7 & 0.8 \\
\hline Creatinine clearance (m//min) & 178 & \pm 18 & 174 & $\$ 14$ & 1.0 \\
\hline Ventilatory days (mean) & 2.7 & \pm 1.2 & 1.6 & \pm 0.6 & 0.003 \\
\hline Sepsis-related deaths (n) & 2 & & 0 & & 0.007 \\
\hline
\end{tabular}

AST, ALT, and bilirubin levels are mean levels from all postoperative time-points. Data are presented as inumber, percentages or mean (standard deviation). Upper gastro-intestinal (Gil) surgery included gastrectomy and pancreatodwodenectomy, and lower $\mathrm{Gl}$ surgery included laparotomy, low anterior resection, left hemicolectomy, subtotal and total colectomy, abdominoperineal resection, and explorative laparotomy. Comparison by chi-square or Student-t test. The APACHE-H and MOF scores were determined from the worst data during the first $24 \mathrm{~h}$ postoperatively 
respect to age, gender, weight, APACHE-score at 24hrs, type of surgery, duration of surgery, and blood loss (Table 1). However, significantly more sepsis-related deaths occurred in patients in the HD+ group and the mean number of days post-operatively on the ventilator was significantly higher in these patients. Moreover, the MOF-score $24 \mathrm{hrs}$ post-operatively was significantly higher in the $\mathrm{HD}+$ group than in the HD-group $(p=0.001)$. Since this was a retrospective analysis of patients originally randomised into three different subgroups, the number of patients in the $\mathrm{HD}+$ and $\mathrm{HD}$ - group divided among the three dopexamine treatment groups were compared. Thus, the included patients represent a group of elective high-risk surgical patients of which a subgroup developed organ dysfunction and complications after a 'silent' interval of 6 days.

\section{Cytokine response to surgery}

The arterial levels of the inflammatory mediators were determined to compare the extent of the systemic inflammation in response to the surgical procedure between the two subgroups. During the postoperative phase mean arterial levels of IL-8, s-ICAM-1, s-TNF-R75, and s-TNF-R55 were significantly higher in patients in the HD+ group (Table 2). This indicates that an increased systemic inflammatory response is present in patients that subsequently developed hepatic organ dysfunction. The pre-operative levels of the inflammatory mediators were not different between the two subgroups. The pre-operative arterial levels in the patients who did not develop hepatic organ dysfunction were similar to the mean post-operative levels (data not shown).

The mean hepatic vein minus arterial blood levels were compared to zero (i.e. no production or uptake) for all patients at all time-points, in order to determine whether significant changes in the kinetics of the inflammatory mediators could be detected. The hepatosplanchnic organs showed a significant production (positive flux) of IL-8 and s-TNF-R75 ( $p=0.04$ and $p=0.0001$ ) and a significant uptake (negative flux) of ICAM-1 and IL-6 ( $p=0.005$ and $p=0.0005$ ) (Table 3$)$. These fluxes accounted for a production of approximately $10 \%$ per minute of the total plasma levels.

The hepatosplanchnic fluxes, indicating the overall amount of inflammatory mediators produced or taken up per minute from the hepatosplanchnic area, were compared between patients developing hepatic dysfunction and in patients with an uneventful surgical procedure. The hepatosplanchnic production of the solubleTNF-receptors and IL-8 was increased 2- to 3 -fold post-operatively in patients in the $H D+$ group $(p=0.02, p=0.02$ and $p=0.04$ for s-TNF-R75, s-TNF-R55, and IL-8, respectively by multiple-way $A N O V A$; dopexamine covariate was $p=0.7, p=1.0$, and $\mathrm{p}=0.2$, for $\mathrm{s}-\mathrm{TNF}-\mathrm{R} 75$, s-TNF-R55, and IL-8, respectively) (Figure 1). The s-TNFRs fluxes were similar between the two subgroups, until 4 h post-operatively for the R75 and until 24 h post-operatively for the R55. At 24-36h post-operatively, the 5TNF-Rs production was significantly higher in the $\mathrm{HD}+$ patients compared to the HD- patients $(p=0.006$ and $p=0.007$ for s-TNF- $r 75$ and R55, respectively). After $36 \mathrm{~h}$ the mean hepatic vein - arterial LL-8 production was significantly increased in 
Table 2 Arterial inflammatory mediator levels in patients who did and did not develop hepatic organ dysfunction

\begin{tabular}{|c|c|c|c|c|c|c|c|}
\hline \multicolumn{2}{|c|}{$\begin{array}{l}\text { Mediators - } \\
\text { post-operative levels }\end{array}$} & \multicolumn{2}{|c|}{$\begin{array}{l}\text { Hepatic dysfunction } \\
\qquad(\mathrm{HD}+)\end{array}$} & \multicolumn{2}{|c|}{$\begin{array}{c}\text { No hepatic dysfunction } \\
\text { (HD) }\end{array}$} & $p_{1}$ & $\mathrm{P}_{2}$ \\
\hline $14-6$ & $(\mathrm{pg} / \mathrm{ml})$ & 334 & \pm 21 & 271 & \pm 20 & 0.5 & 0.5 \\
\hline IL-6 & $(\mathrm{pg} / \mathrm{ml})$ & 74.7 & \pm 9.0 & 44.9 & \pm 3.1 & 0.3 & 0.005 \\
\hline S- $\mid C A M-1$ & $(\mathrm{ng} / \mathrm{ml})$ & 47.2 & \pm 4.0 & 38.0 & \pm 2.0 & 0.2 & 0.03 \\
\hline S-E-selectin & $(\mathrm{ng} / \mathrm{ml})$ & 31.8 & \pm 2.5 & 30.0 & \pm 1.7 & 0.2 & 0.8 \\
\hline TNF-R-75 & $(\mathrm{ng} / \mathrm{ml})$ & 3.6 & \pm 0.11 & 2.6 & \pm 0.2 & 0.048 & 0.003 \\
\hline TNF-R-55 & $(\mathrm{ng} / \mathrm{ml})$ & 13 & \pm 0.06 & 0.9 & \pm 0.03 & 0.4 & 0.004 \\
\hline \multicolumn{2}{|c|}{$\begin{array}{l}\text { Mediators - } \\
\text { pre-operative levels }\end{array}$} & \multicolumn{2}{|c|}{$\begin{array}{l}\text { Hepatic dysfunction } \\
\qquad(\mathrm{HD}+)\end{array}$} & \multicolumn{2}{|c|}{$\begin{array}{l}\text { No hepatic dysfunction } \\
\text { (HD) })\end{array}$} & \multicolumn{2}{|c|}{ p-value } \\
\hline$I L-6$ & $(\mathrm{pg} / \mathrm{ml})$ & 335 & \pm 49 & 268 & \pm 65 & 0 & \\
\hline $1 \mathrm{~L}-8$ & $(\mathrm{pg} / \mathrm{ml})$ & 47.8 & \pm 17.1 & 42.0 & \pm 7.5 & 0 & \\
\hline s-|CAM-1 & $(\mathrm{ng} / \mathrm{ml})$ & 36.1 & \pm 4.8 & $39: 3$ & \pm 3.3 & 0 & \\
\hline S-Es-selectin & $(\mathrm{ng} / \mathrm{ml})$ & 36.7 & \pm 5.8 & 29.7 & \pm 3.7 & 0 & \\
\hline TNF-R-75 & $(\mathrm{ng} / \mathrm{mL})$ & 2.7 & \pm 0.3 & 2.0 & \pm 0.2 & & \\
\hline TNF-R-55 & $(\mathrm{ng} / \mathrm{mL})$ & 0.7 & \pm 0.06 & 0.9 & \pm 0.09 & 0 & \\
\hline
\end{tabular}

Data are presented as mean values \pm SEM from all post-operative and pre-operative time-points. $\|-6_{\text {in }}$ interleukin- 6 ; s-ICAM-1, soluble intracellular adhesion molecule-1; s-TNF-r-75, soluble-tumor necrosis factor-receptor-75. Comparison of post-operative levells by multiple-way ANOVA. The $p_{1}$ value indicates the significance of the within-subjecl analysis (timerelated influencel and $p_{2}$ indicates the significance of the interaction between repeated measures $x$ presence of hepatic organ dysifunction. Comparison of pre-operative levels by Mann-Whitney U test

Table 3 Overall hepatic vein minus arterial plasma differences of inflammatory mediators compared to zero

\begin{tabular}{lllll}
\hline Mediators - post-operative levels & \multicolumn{2}{c}{ Plasma levels } & p-walue \\
\hline IL-6 & $(\mathrm{ng} / \mathrm{ml})$ & -0.02 & \pm 0.006 & 0.0005 \\
IL-8 & $(\mathrm{pg} / \mathrm{ml})$ & $2.7 \times 10^{-3}$ & $\pm 1.5 \times 10^{-3}$ & 0.04 \\
s-ICAM-1 & $(\mathrm{ng} / \mathrm{ml})$ & -0.20 & \pm 0.07 & 0.005 \\
s-E-selectin & $(\mathrm{ng} / \mathrm{mll})$ & 0.41 & \pm 0.49 & 0.2 \\
s-TNF-r-75 & $(\mathrm{ng} / \mathrm{ml})$ & 0.21 & \pm 0.03 & $<0.0001$ \\
5-TNF-r-55 & $(\mathrm{ng} / \mathrm{ml})$ & 0.02 & \pm 0.02 & $0 . \|$ \\
\hline
\end{tabular}

Data are presented as mean \pm SEM from all post-operative time-points for all patients combined. The lewels of inflammatory levels were compared to zero using the one-sample Komolgorov-Smimov test. A significant level indicales that the mean level is significantly higher (positive values) or significantly lower than zero (negative values), indicating significant production or uptake from the hepatosplanchnic area. IL-6, interleukin-6; 5-ICAM-1, soluble intracellular adhesion molecule-1; $5-T$ NF-r- $75_{i}$ soluble-tumor necrosis factor-receptor-75. 

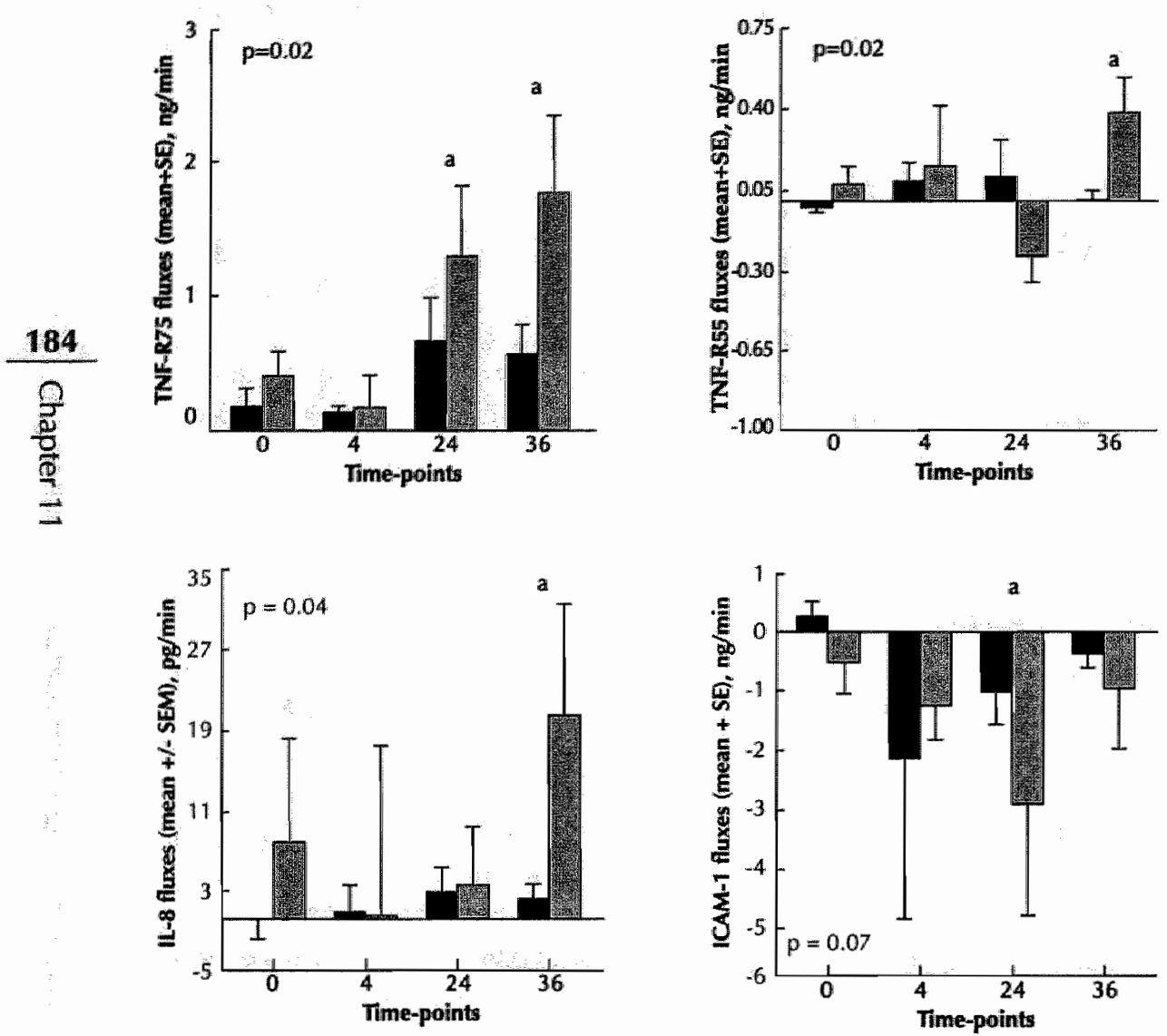

Mean changes in s-TNF-R75, R55, IL-8 and ICAM-1 filuxes over the hepatosplanchnic area are depicted for patients without the development of post-operative hepatic dysiunction (HD), solid bars) and patients with the development of post-operative hepatic dystunction ( $\mathrm{HD}+$, shaded bars). A value above zero (positive net balancel indicates net efflux ar release and a value below zero (negative net ballance) represents net influx or uptake. 0 is pre-operative time-point, 4,24 , and 36 indicates the respective post-operative time-points. The cytokine fluxes between the subgroups were compared using multipleway ANOVA for correction of lime-related influences with the dopexamine treatment as cowariate. Individual time-points were analysed using the Mann-Whitney $U$ test and a indicates $p<0.05$ for this ind ividual time-point.

\section{Figure 1a-d Hepatosplanchnic fluxes of inflammatory mediators in patients who did and did not develop hepatic organ dysfunction}

patients in the HD+ group ( $p=0.005$ ) (Figure 1). In contrast, the hepatosplanchic production or clearance of IL-6, and E-selectin were similar between the two subgroups for all time-points combined (data not shown).

The postoperative s-ICAM-1 uptake in the hepatosplanchnic area tended to be higher in the HD+ group compared to the uptake in the HD-group $(p=0.07)$ (Figure 1 ). 

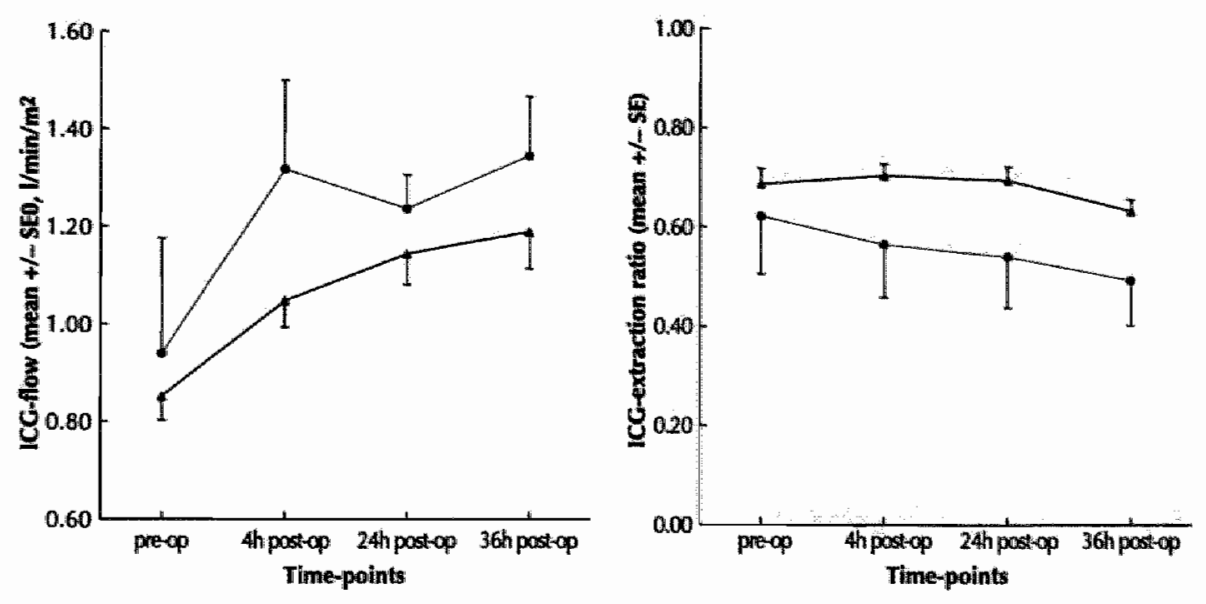

Changes in ICG-extraction $(\mathrm{p}=0.03)$ and ICG-flow $\left(/ / \mathrm{min} / \mathrm{m}^{2}\right)(\mathrm{p}=0.01)$ of the liver are compared between patients withoult the development of post-operative hepatic dysfunction (HD-, - - ) and patients with the development of post-operative hepatic dysfunction (HD+, ……). Data are presented as mean (SE). 'Pre-OP' is pre-operative measurement, 'th post-op' is $4 \mathrm{~h}$ post-operatively.

Figure 2 Peri-operative liver ICG-extraction and ICG-flow in patients who did and did not develop hepatic organ dysfunction

In the HD+ group the increase in hepatic vein- arterial uptake at $24 \mathrm{~h}$ postoperatively compared to pre-operatively was $170 \%(p=0.04)$.

No relationship between the presence of metastases or time of surgery and the magnitude of mediator flux could be found in this study (data not presented).

\section{Haemodynamic response to surgery}

The global haemodynamic course post-operatively was investigated in comparison with the changes in regional perfusion and indicated that no differences could be detected in pre-operative measurements of the global haemodynamic parameters between the two subgroups (data not shown). The heart rate and arterial lactate levels increased to a significantly higher level during surgery and in the postoperative period in the HD+ group $i p=0.01$ and $p=0.001$, respectively (Table 4)). Arterial lactate levels returned to normal levels within $24 \mathrm{hrs}$ after surgery. In patients in the HD- group arterial lactate levels only increased marginally above baseline levels. Blood creatinine levels were similar pre-operatively $(79.5 \pm 12.3$ vs. $71.2 \pm 10.2 \mathrm{mmol} / \mathrm{l}$ ). No differences in post-operative kidney function could be detected between the 2 subgroups (Table 1 ). 


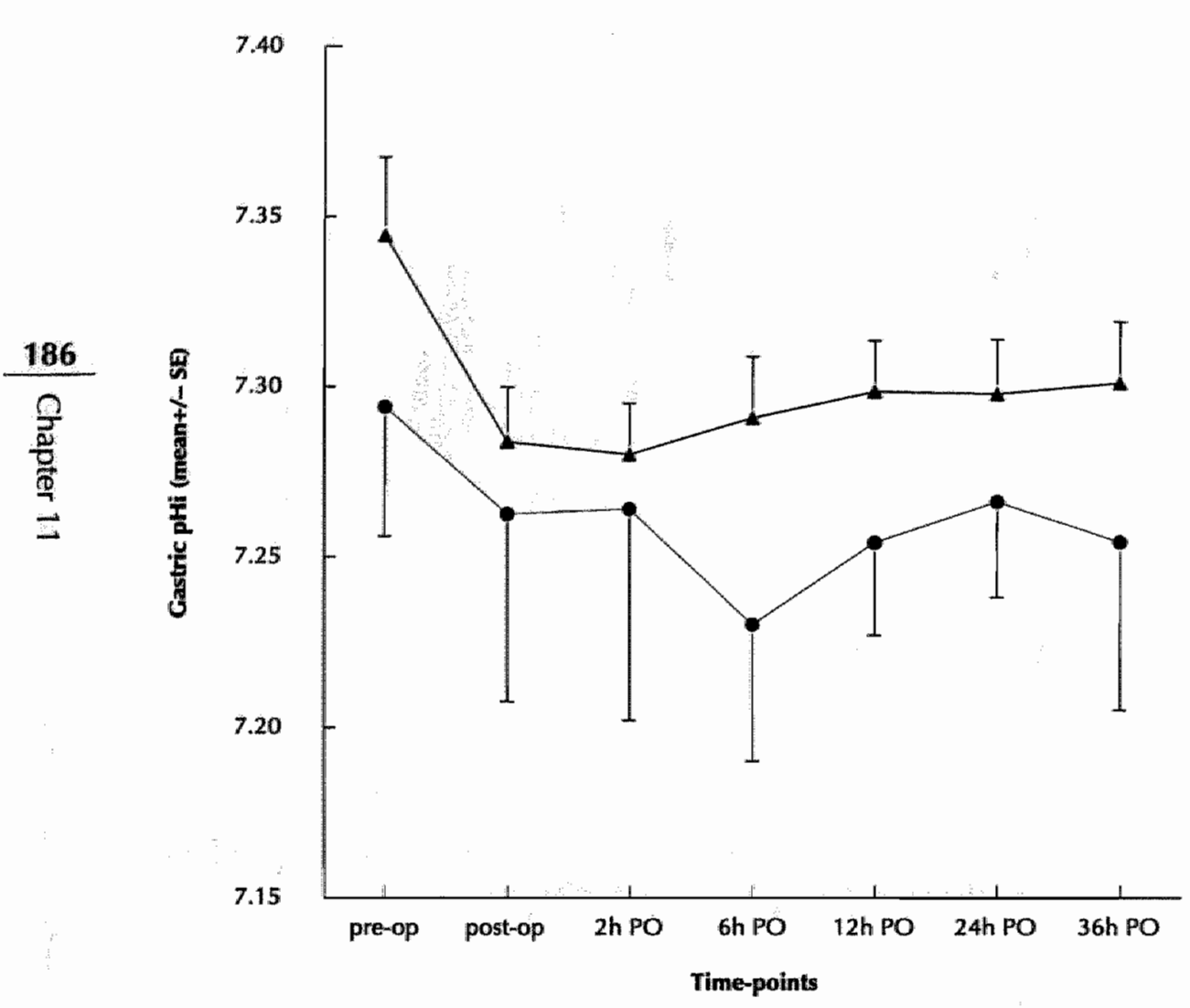

Changes in gastric intramucosal $\mathrm{pHil}(\mathrm{p}=0.04)$ are depicted for patients without the development of post-operative hepatic

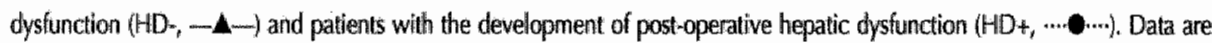
presented as mean (SE). "Pre-op' is pre-operative measurement, 'post-op' is post-operatively, " $2 \mathrm{~h} \mathrm{PO}$ ' is $2 \mathrm{~h}$ post-operatively.

Figure 3 Peri-operative pHi-levels in patients who did and did not develop hepatic organ dysfunction

Regional perfusion parameters were not significantly different pre-operatively in total hepatosplanchnic blood flow and gastric $\mathrm{pHi}(\mathrm{p}=0.1$ and $\mathrm{p}=0.2$, respectively). A significantly higher total hepatosplanchnic blood fllow from four to 36 hrs postoperatively $(p=0.01)$ and a significantly lower hepatic extraction of ICG occurred in the HD+ group $(p=0.03)$ (Figure 2). After 36 h post-operatively, a $20 \%$ reduction in ICG-extraction was observed. This was accompanied by a decrease in pHi to a significantly lower level from six to 36 hrs postoperatively in the HD+ group $(\mathrm{p}=0.04)$ (Figure 3 ), whereas bicarbonate levels and arterial $\mathrm{pH}$ were simitar between the two subgroups $(24.0 \pm 2.9$ vs. $24.0 \pm 2.6, p=0.5)$ and $(7.399 \pm 0.04$ vs. $7.406 \pm 0.03 ; p=0.2)$. In figure 4 the relationship between the hepatic vein minus ( - ) arterial differences for IL-6 and IL-8 are depicted against the pHi values. 

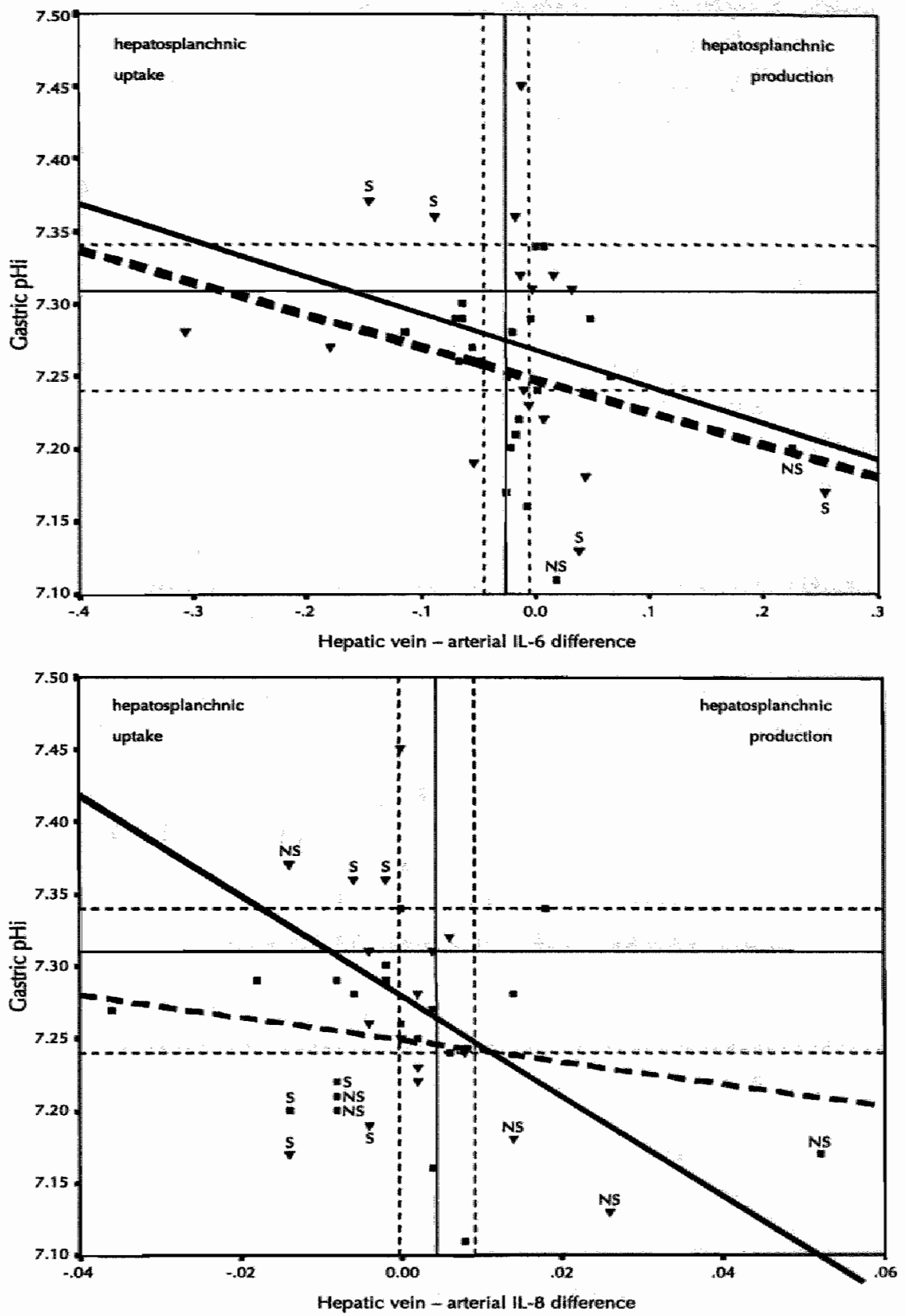

Data represents correlation between hepatic vein - arterial cytokine differences and gastric intramucosal pHi levels for patients with an uneventful sumgical procedure $(--\pi)$, and patients with complicated surgery $(\cdots, / \mathrm{V})$. For all patients zero lL-6 and zero IL-8 (vertical _- line) differences correlate with a intramucosal pHli of 7.290 thorizontal __ line), A significant correlation between IL-6 differences and pHil was found in patients with both complicated and uncomplicated surgery and for It-8 differences and $\mathrm{pH} \mathrm{H}$ in patients with complicated surgery. $5=$ survivor, $\mathrm{NS}=$ non-surviwor.

Figure 4 Relation between gastric intramucosal pHi and hepatic vein - arterial plasma cytokine differences in patients with sepsis 
These data show that at lower $\mathrm{pHi}$ the hepatic vein - arterial difference increases to reach positive values. This means that there is more production in the hepatosplanchnic organs than clearance. In patients with both a complicated and uncomplicated surgical procedure for IL6 the correlation was $-0.40(p=0.03)$ and in patients with complicated surgery for IL-8 the correlation was $-0.40(p=0.04)$.

\section{Discussion}

This study investigated the role of the hepatosplanchnic area and the inflammatory response to surgery, in the development of organ failure. This is to our knowledge the first study in elective high-risk surgical patients that detected production of inflammatory mediators in the hepatosplanchnic area prior to the development of clinical hepatic dysfunction. Organ dysfunction is an important cause for clinical deterioration on the surgical intensive care and is considered the end point of damage caused by the inflammatory response. Moreover, the liver has a central role in supporting the defense systems of the body, and an impending liver dysfunction is an ominous sign in critically ill patients (24). Therefore, the development of post-operative hepatic organ dysfunction was used retrospectively as criterium for dividing the patient population. The criteria for hepatic organ dysfunction in our study were identical to that in the study by Douzinas et al. (9). The clinical consequences of the presence of post-operative hepatic organ dysfunction were emphasized by the increased sepsis-related mortality and prolonged ventilatory need. Previously, Douzinas et al. found an increased cytokine production from the hepatosplanchnic area in patients with established sepsis and MOF (9). Two other clinical studies investigated the portal vein levels (intestinal production) after surgery. In a study by Cabie et al. portal vein levels of TNF were higher than systemic levels after reperfusion during abdominal aortic surgery in man (4). In the study by Maeba et al., portal vein IL-6 levels were from 3 to $12 \mathrm{hrs}$ after the commencement of digestive surgery higher than the systemic levels (25).

In this study, the hepatosplanchnic area appeared to be an important site for the increased and prolonged production of IL-8, s-TNF-R75 and R55 in patients developing hepatic organ dysfunction. Patients with an uncomplicated surgical outcome had a lower, shorter lasting, production of these inflammatory mediators. The levels of the soluble TNF-receptors give an indirect indication of the activitiy of TNF, because soluble TNF-receptors can be constantly found in the circulation during critical illness even when TNF activity is undetectable (26). The soluble TNF-receptors are released in response to production of TNF. This can affect TNF activity not only by competing with the cell -associated TNF receptors, thereby interfering with its binding to cells, but also by stabilizing its structure and preserving the activity of TNF, thus augmenting the effects of TNF (27). Irrespective of the effects of the TNF-receptors on the TNF activity, the increased production 
or release of the TNF-receptors found in our study indicates the presence of a prolonged inflammatory response in the hepatosplanchnic area in patients who subsequently developed post-operative organ dysfunction. A prolonged increase of levels of TNF and its receptors has previously been associated with increased mortality and morbidity $(9,23,28,29)$. The levels of $s-T N F-R s$ found in our study were comparable to levels found in other studies including patients with complicated surgery $(28,30)$.

Previous studies indicated that TNF-receptors are cleared from the circulation by the kidneys (23). Therefore, the urinary flow rate and creatinine clearance were used as markers for kidney function. These markers remained similar in both study groups throughout the post-operative period. Therefore, differences in kidney clearance are not a likely explanation for the difference in plasma levels of the TNF-receptors between the groups.

In addition to our initial finding that an increased production of inflammatory mediators from the hepatosplanchnic area was found in post-operative patients at risk of developing organ dysfunction, a substantial peri-operative uptake of s-ICAM-1 from the circulation by the hepatosplanchnic organs was found in this study. Systemic levels of S-ICAM were significantly higher in patients who developed organ dysfunction compared to patients with uneventful surgery. These levels were comparable with blood levels of s-ICAM in other studies $(31,32)$. An uptake of $5-I C A M-1$ was previously found by Miwa et al. in cardiac tissue, and this uptake was associated with decreased cardiac function (33). It was hypothesized that this was due to entrapment of S-ICAM-1 in the ischaemic phase of coronary spasm as result of the binding of s-ICAM-1 to leukocytes, with subsequent activation and adhesion of the leukocytes to the endothelium and regulation of the synthesis of acute-phase proteins. The increased production of $\mathrm{IL}-8$, preceding the development of the hepatic organ dysfunction, seems to emphasize the importance of leucocyte activation in this process, because IL- 8 is considered an important inducer of neutrophil chemotaxis and activation (34). The increased plasma levels of IL-8 found in our study in patients with hepatic organ dysfunction were comparable to levels found in surviving patients with severe sepsis (35). Although $\| \mathrm{L}-6$ has been shown to be related to IL-8 production during decreased splanchnic perfusion (12), this study failed to show a difference in IL-6 flux over the hepatosplanchnic area between the two subgroups, although the overall hepatic vein minus arterial plasma differences of inflammatory mediators were significantly lower than zero for all patients combined. IL-6 is considered an important stimulator of the acute phase response in the liver (36). However, other studies found the expression of C-reactive protein without the involvement of IL-6 (37) or the mediation of the acute-phase response by IL-8, unrelated to IL-6 (38). Whether in our study the induction of the inflammatory response without a significant flux of IL-6 over the hepatosplanchnic area is due to methodological problems or an uninvolvement of IL-6 remains to be seen.

For the first time an uptake of s-ICAM is described in the hepatosplanchnic area and could be related to the development of hepatic organ failure. The lack of differences in levels of $s$-E-selectin may suggest that this process was not 
accompanied by activation of endothelial cells in these patients.

However, this study has some limitations. The method of evaluating hepatosplanchnic mediator flux used in our study does not allow detection of cytokine production or uptake in the liver or gastro-intestinal organs separately. Such an intrasplanchnic production may be missed unless splanchnic production is higher than the compensatory hepatic uptake. Data concerning the inter-organ flux of cytokines in animal and human subjects are limited. McGuiness et al. found hepatic release of TNF and did not find production of TNF in nonhepatic splanchnic tissues after endotoxin infusion in conscious dogs, although this was infused directly in the portal vein (39). The aim of our study was primarily to examine if it was possible to detect differences in total hepatosplanchnic mediator metabolism at such an early phase after elective surgery, which could be related to outcome. This area has been very little explored in the past and this retrospective pilot study should be regarded as a hypothesis generating study. Several confounding factors may be suggested in the data presented. Firstly the number of patients with metastases seem to be higher in the group developing hepatic dysfunction. Patients operated upon for metastasized malignancies are known to have higher levels of inflammatory mediators (40). However, when comparing the patients with and without metastases, no differences in mediator flux could be found. The same accounted for the time of surgery, which showed a tendency to be longer in the patients developing hepatic dysfunction. No relationship, however, could be found between the time of surgery and the magnitude of the inflammatory response in the hepatosplanchnic area.

A disturbed regional perfusion is another factor that may be related to an increase in the regional inflammatory response and that could contribute to the development of organ failure after surgery. In this study the patients who developed post-operative organ dysfunction had an increased total hepatosplanchnic blood flow from $4 \mathrm{~h}$ post-operatively and a significantly lower gastric $\mathrm{pH}$ from $6 \mathrm{~h}$ post-operatively compared to patients having an uneventful post-operative outcome. This could not be accounted for by changes in the globally measured haemodynamic variables. Indeed, a significant relationship could be demonstrated in the patients with a complicated surgical procedure between the gastric $\mathrm{pHi}$ and $\mathrm{IL}-6$ and $\mathrm{II}-8$ fluxes in the hepatosplanchnic area. Whether this indicates that a causal relationship between splanchnic perfusion and the splanchnic inflammatory response in humans needs to be determined further and should be considered hypothesis generating at this point. Although several experimental studies (41) support this hypothesis, some points from our data need to be clarified. The increase in total hepatosplanchnic blood flow seemed to be in contradiction to the apparent hypoperfusion indicated by the decreased gastric pHi. This has been demonstrated before (42) and may be related to the presence of heterogenous blood flow within the hepatosplanchnic area, with the increase in total hepatosplanchnic flow being a compensatory response. The implications of this finding in our study is not clear. In addition, the intramucosal pHi is also determined by the mucosal cell metabolism and may therefore be influenced by factors such as oxygen consumption, haemoglobin and $\mathrm{pH}$. 
In conclusion, the presence of an inflammatory response in the hepatospllanchnic area seems to play an important role in the development of organ failure after high-risk surgery. Although this is a retrospective study in a small group of patients we believe it is important, since it shows that in a subgroup of elective surgical patients hepatosplanchnic production of inflammatory mediators appeared to predict subsequent hepatic dysfunction. Further studies investigating inter-organ fluxes of cytokines in experimental and clinical settings are required to gain more insight into the exact kinetics of cytokines in the hepatosplanchnic organs and its relation to the development of postoperative complications. 


\section{REFERENCES}

1. van Berge Henegouwen $M I_{t}$ van der Poll $\mathrm{T}$, van Deventer $\$ \mathrm{H}$ : Peritoneal cytokine release after elective gastrointestinal surgery and postoperative complications. Am / Surg 175: 311-316, 1998.

2. Partrick DA, Moore FA, Moore EE, Biffl WL, Sauaia A, Barnett CC]: Jack A. Barney Resident Research Award winner. The inflamimatory profile of interleukin- 6 , interleukin- 8 , and soluble intercellular adhesion molecule 1 in postinjury multiple organ failure. Am J Surg 172: 425-429, 1996.

3. Biffll $W L$, Moore EE, Moore FA, Peterson VM: Interleukin-6 in the injured patient. Marker of injury or mediator of inflammation? Ann Surg 224: 647-664, 1996.

4. Cabie $A_{*}$ Karkas J-C, Fitting $C_{x}$ Laurain $C_{1}$ Cormier J-M, Carlet l, Cavaillon J-M: High levels of TNF-a during abdominal surgery in man. Cytokine 5: 448-453, 1993.

5. Tang Gl, Kuo CD, Yen TC, Kuo HS, Chan KH, Yien HW, Lee TY: Perioperative plasma concentrations of tumor necrosis factor-alpha and interleukin- 6 in infected patients. Crit Care Med 24: 423-428, 1996.

6. Aderka D, Sorkine P, Adu-Adib S, Lev D, Setton A, Cope AP, Wallach D, Klausner JM: Shedding kinetics of soluble tumor necrosis factor (TNF) receptor after systemic TNF leaking during isolated limb perfusion. Relevance to the pathophysiology of septic shock. / Clin Invest 101: 650-659, 1998.

7. Bone RC: Towards a theory regarding the pathogenesis of the systemic inflammatory response syndrome: what we do and do not know about cytokine regulation. Crit Care Med 24: 163-172, 1996.

8. Biffl WL, Moore EE, Moore FA: Gut-derived mediators of multiple organ failure: plateletactivating factor and interleukin-6. Br J Hosp Med 54: 134-138, 1995.

9. Douzinas EE, Tsidemiadou PD, Pitaridis MT, Andrianakis ॥, Bobota-Chloraki A, Katsouyanni Kr Syras $D$, Malagari $K$, Roussos $C$ : The regional production of cytokines and lactate in sepsisrelated multiple organ failure. AmJ Respir Crit Care Med 155: 53-59, 1997.

10. Maynard ND, Bihari D), Dalton RN, Beale R, Smithies MN, Mason RC: Liver function and splanchnic ischemia in critically ill patients. Chest 111: 180-187, 1997.

11. Maynard ND, Bihari DJ, Dalton RN, Smithies MN, RC Mason. Increasing splanchnic blood flow in the critically III. Chest 108: 1646-1654, 1995.

12. Donati A, Battisti D, Rechioni A, Paoletti P, Conti G, Caporelli S, Adrario E, Pelaia P, Pietropaoli $P$ : Predictive value of interleukin-6 (IL-6), interleukin-8 (IL-8) and gastric intramucosal $\mathrm{pH}(\mathrm{pH}-\mathrm{i})$ in major abdominal surgery. Intensive Care Med 24: 3351998.

13. Takala I. Meier-Hellmann A, Eddleston JM, Hulstaert PF, Sramek $V_{j}$ on behalf of the European Multicenter study group on dopexamine in major abdominal surgery. Effect of dopexamine on outcome after major abdominal surgery: a prospective, randomized, controlled multi-center study. Crit Care Med 28: 3417-3423, 1998.

14. Bland RD, Shoemaker WC, Shabot MM: Physiologic monitoring goals for the critically ill patient. Surg Gynecol Obstet 147:833-841, 1978.

15. Doglio GR, Pusajo JF, Egurrola MA, Bonfigli GC, Parra $C_{*}$ Vetere L, Hernandez MS, Fernandez

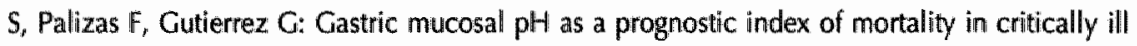
patilents. Crit Care Med 19: 1037-1040, 1991.

16. Uusaro $A$, Ruokonen $E$, Takala J. Estimation of splanchmic blood flow by the Fick principle in man and problems in the use of indocyanine green. Cardiovasc Res 30: 106-112, 1995. 
17. Goris R/, te Boekhorst TP, Nuytinck IK, Gimbrere IS; Mwthle-organ ailure. Ceneralzed autodestructive inflammation? Arch Surg 120.1109.1115, 1985.

18. Dentener MA, Bazil V, von Asmuth EJU, Ceska $\mathrm{M}$, Bumman WA: hovolvement of CO/4 in Hipopolysaccharide-induced tumor necrosis factor-a, $14-6$ and $4-8$ release by twaman monocytes and alveolar macrophages. I Immunol 150: 2885-2891, 1993.

19. Bouma MG, Stad RK, van den Wildenberg FAM, Buurman WA: Differential regulatory effects of adenosine on cytokine release by activated human monocytes. I mmunol 153:4159-4168, 1994 .

20. Bouma $\mathrm{MG}_{\mathrm{g}}$ Laan MP, Dentener MA, Butuman WA. Analysis of soluble adhesion molecules. In: Immunochemistry 2: A practical approach, edited by Johnstone AP, and Tumer MW: Oxford; Oxford Uniwersity Press, 1997, p. 181-196.

21. Leeuwenberg IF, Jeunhomme TM, Butuman WA: Slow release of soluble TNF receptors by monocytes in vitro. I Immunol 152: 4036-4043, 1994.

22. Thornhill MH, Wellicome SM, Mahiouz DL, Lanchbury ISS, Kyan-Aung U, Haskard DO Tumor necrosis factor combines with $\mathrm{LL}-4$ and IFNg to selectively enhance endathelial cell adhesiveness for $T$ cells. The contribution of vascular cell adhesion molecule-1-dependent amd -independent binding mechanisms. I Immunol 146: 592-598, 1991.

23. Froon AHM, Bemelmans MHA, Greve IWM, van der Linden Cl, Butrman WA. Increased plasma levels of soluble tumor necrosis factor receptors in sepsis syndrome: correlation with plasma creatinine. Crit Care Med 22: 803-809, 1994.

24. Hawker F: Liver dysfunction in criticall illness. Anaesth. Intensive, Care 19: 165-181, 1991.

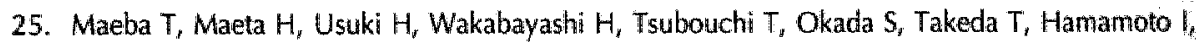
Okano $K$, ishimura $K$ : Increase in portal blood interleukin- 6 soon atter the commencement of digestive surgery. Surg Today 26: 890-894, 1996.

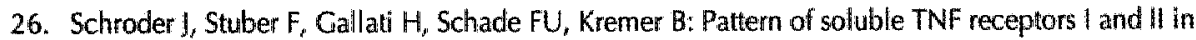
sepsis. Infection 23: 143-148, 1995.

27. Aderka D, Engelmann H, Maor Y, Brakebusch C, Wallach D. Stabilization of the bioactivity of tumor necrosis factor by its soluble receptors. I Exp Med 175: 323-329, 1992.

28. Kimura $F$, Miyazaki $M$, Suwa $T$, Sugiura $T$, Shinoda $T$, Itoh $H$, Ambiru $S$, Shimizu $H$, Nakagawa $K$ : Plasma concentration of cytokine antagonists in patients with infection following liver resection. Br J Surg 85: 1631-1635, 1998.

29. de Beaux $A C$, Goldie AS, Ross JA, Carter DC, Fearon $K C H$. Serum concentrations of inflammatory mediators related to organ failure in patients with acute pancreatitis. Br J Surg B3." 349-353, 1996.

30. Pilz G, Fraunberger P, Appel R, Kreuzer E, Werdan K, Walli A, Seidel D: Early prediction of outcome in score-identified, postcardiac surgical patients at high risk for sepsis, using soluble tumor necrosis factor receptor-55 concentrations. Crit Care Med 24: 596-600, 1996.

31. Law MM, Cryer HG, Abraham E: Elevated levels of soluble ICAM-1 correlate with the development of multiple organ failure in severly injured trauma patients. J Trauma 37: 100-108, 1994.

32. van Deventer SJH, Pajkrt D: Neutrophil adhesion moleculles and MOF, Intensive Care Med 23: $487-488,1997$.

33. Miwa $K$, Igawa $A$, Inoue $H$ : Soluble E-selectim, $I C A M-1$ and VCAM-1 levels in systemic and coronary circulation in patients with variant angina. Cardiovasc Res 36: 37-44, 1997.

34. Botha AJ, Moore FA, Moore EE, Peterson VM, Silliman CC, Groode AW: Sequentiall systemic platelet-activating factor and interleukin 8 primes neutrophils in patients at risk of multiple organ failure. Br J Surg 83: 1407-1412, 1996. 
35. Marty $C$, Misset $B$, Tamion $F_{r}$ Fitting $C$, Carlet $\mathbb{J}$, Cavaillon J-M: Circulating interleukin-8 concentrations in patients with multiple organ failure of septic and nonseptic origin. Crit Care Med 22:673-679, 1994.

36. Castell IV, Andus $T$, Kunz D, Heinrich PC: Inteleukin-6. The major regulator of acute-phase protein syntehesis in man and rat. Ann M Y.Acad.Sci 557: 87-99, 1989.

37. Ku NO, Mortensen RF: The mouse C-reactive protein (CRP) gene is expressed in response to IL-1 but not IL-6. Cytokine 5: 319-326, 1993.

38. Wigmore SI, Fearon KCH, Maingay JP, Lai PBS, Ross JA: Interleukin-8 can mediate acute-phase protein production by isolated human hepatocytes. Am I Physiol 273: E720-E7261997.

39. McGuinness OP, Lacy DB, Ejiolo ل, Bagby GG. Hepatic rellease of tumor necrosis factor in the endotoxin-treated consilous dog. Shock 5 ; 344-348, 1996.

40. Richế $\mathrm{F}$, Dosquet $\mathrm{C}$, Panis $\mathrm{Y}$, Valleur $\mathrm{P}$, Lailsné $\mathrm{M}-\mathrm{J}$, Briard $\mathrm{C}$, Wautier $\mathrm{J}-\mathrm{L}$ : Levels of portal and systemic blood cytokines after colectomy in patients with carcinoma or Crohn's disease. I Am Coll Surg 180: 718-724, 1995.

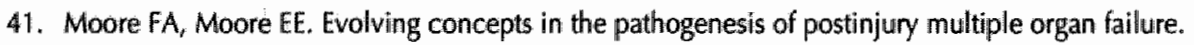
Surg Clin North Am 1995; 75(2):257-277.

42. Uusaro A, Ruokonen $E$, Takala J: Gastric mucosal pH does not reflect changes in splanchnic blood flow after cardiac surgery. Br I Anaesth 74: 149-154, 1995. 


\section{CHAPTER 12}

Prolonged nitric oxide synthase- 2 inhibition during hyperdynamic endoloxaemia in the pig increases mortality and deterionates hepatosplanchnic perfusion and metabolism 


\section{ABSTRACT}

Objective: To investigate the hypothesis that prolonged selective inducible nitric oxide synthase (NOS2) inhibition during hyperdynamic endotoxaemia in the pig deteriorates hepatosplanchnic perfusion and metabolism, and reduces survival.

Design: Randomised, controlled trial.

Setting. University research laboratory.

Subjects: Female pigs (20-25 kg) received $3 \mu \mathrm{g} / \mathrm{kg} / \mathrm{h}$ lipopolysaccharide endotoxin

(LPS) intravenously and saline resuscitation during $24 \mathrm{hrs}, 14$ days after surgical implantation of catheters.

Interventions: Intravenous administration of the selective NOS2 inhibitor amino-ethyl-isothiourea (AE-1TU; $10 \mathrm{mg} / \mathrm{kg} / \mathrm{h}, \mathrm{n}=6)$ or saline $(0.9 \% ; n=7)$ between $4 \mathrm{hrs}$ after starting and $24 \mathrm{hrs}$ after stopping the LPS administration.

Measurements and results: NO production, determined by primed, stable isotope infusion of ${ }^{15} \mathrm{~N}_{2}$-Arginine and ${ }^{13} \mathrm{C}-{ }^{2} \mathrm{H}_{2}$-Citrulline, was significantly reduced during AE-ITU treatment. Similarly, arginine and citrulline appearance rates and arginime de novo synthesis were significantly lower in AE-ITU treated animals. Mortality was significantly higher during AE-ITU treatment. Hepatosplanchnic perfusion was measured using primed-continuous infusion of para-aminohippuric acid across the portal-drained viscera (PDV) and liver and by intramucosal $\mathrm{pCO}_{2}$. PDV flow was significantly increased during AE-ITU treatment, as were hepatic oxygen consumption and extraction during LPS administration. In the 24 hrs following cessation of endotoxin infusion, PDV flow further increased 1.7-fold, whereas that through the hepatic artery declined to $50 \%$. As a result, total blood flow through the liver did not change, but oxygen delivery decreased to $50 \%$. AE-liTU treatment decreased splanchnic intramucosal $\mathrm{pCO}_{2}$ significantly during endotoxin infusion, but this effect disappeared after discontinuing LPS. AE-ITU treatment increased the lactate/pyruvate ratio during and after LPS infusion.

Conclusion: Selective inhibition of NOS2 improves hepatosplanchnic perfusion and metabolism during the initial phase of endotoxaemia. However, prolonged post-endotoxaemic inhibition has detrimental effects on these parameters and is associated with a dramatic reduction in survival. 


\section{INTRODUCTION}

Sepsis and endotoxaemia are characterised by systemic hypotension and vascular hyporeactivity to vasopressor agents (1). Recent developments in the treatment of shock in sepsis indicate an important role for the nitric oxide (NO) (2) that is produced by the cytokine-inducible nitric=oxide synthase-2 isoform (NOS2) $(3,4)$. The increased formation of NO by NOS2 probably contributes to the vascular dilatation that causes hypotension (5). Inhibition of the excess NO formation by inhibiting NOS2 activity was therefore proposed as a treatment for endotoxic shock. However, inhibition of NOS activity in patients suffering from sepsis using the non-specific NOS inhibitor $L-N{ }^{G}$-methylarginine-hydrocholine was associated with an increased mortality rate (6).

Under physiological conditions, the NO that is produced by the NOS1 and NOS3 isoforms regulates vascular tone and blood pressure, blood-flow distribution in organs, and neurotransmission (7). During sepsis, endotoxin reduces NOS1 and NOS3-mediated NO production (8). Furthermore, substantial experimental evidence indicates that inhibition of NOS1 and NOS3 activity during sepsis increases hepatic and splanchnic damage (9). Hypothetically, upregulation of NOS2 during sepsis can compensate for the downregulation of NOS1 and NOS3 with respect to organ perfusion. Untimely and uncontrolled inhibition of NOS during septic shock may, therefore, be responsible for the persistent and uncorrectable splanchnic ischaemia frequently seen in such experiments $(10)$. Accordingly, the use of selective NOS2 inhibitors has been controversial thus far because of adverse effects on hepatic perfusion $(11,12)$. In line with these arguments, we wished to test the hypothesis that selective NOS2 inhibition has

a detrimental effect on hepatosplanchnic perfusion and metabolism, especially during prolonged use. To that end, we developed a sub-acute hypermetabolic animal model that resembled the hyperdynamic septic state seen in patients with sepsis and multiple-organ failure (13). Firstly, endotoxin was administered by infusion rather than as a bolus. Secondly, the inhibition of NOS2 was started $4 \mathrm{~h}$ after the start of the endotoxin infusion, instead of prior to the endotoxin infusion. Finally, the inhibition of NOS2 was continued after discontinuing the endotoxin infusion to simulate the effects of ongoing NO inhibition during the beginning of recovery in the clinical situation.

\section{MATERIALS AND METHODS}

\section{Animals}

Female crossbred pigs (Yorkshire $x$ Dutch Landrace; 20-25 kg) were individually housed, had free access to water and received $1 \mathrm{~kg}$ of regular pig feed $(16 \%$ 
crude protein; Landbouwbelang, Roermond, the Netherlands) daily in the morning, which supported a growth rate of 300 gram per day. The Animal Ethics Committee of Maastricht University approved the study.

\section{Surgical Procedure}

Animals were fasted overnight. After the pigs were premedicated with $10 \mathrm{mg} / \mathrm{kg}$ azoperone (Stresnil, Janssen Pharmaceutica, Besse, Belgium) intramuscularly, anaesthesia was induced with a mixture of $\mathrm{N}_{2} \mathrm{O} / \mathrm{O}_{2}(1: 2$, by voll) and halothane $(0.8 \%)$. After intubation, the pigs were intravenously administered $6.25 \mathrm{mg} / \mathrm{kg}$ lincornycin (A.U.V., Cuyk, The Netherlands) as bactericidal prophylaxis and 12.5 $\mathrm{mg} / \mathrm{kg}$ spectinomycin (A.U.V.) as bacteriostatic prophylaxis. Flunixine $50 \mathrm{mg} / \mathrm{kg}$ (finadyne Schering-Ploegh, Brussels, Belgium) was administered intravenously for postoperative anal|gesia. During surgery, anaesthesia was maintained with a mixture of $\mathrm{N}_{2} \mathrm{O} / \mathrm{O}_{2}$ and halothane. Hydratation was maintained by intravenous administration of Ringer's lactate (Lactetrol, $15 \mathrm{ml} / \mathrm{kg} / \mathrm{h}$, Aesculaap, Boxtel, The Netherlands).

After midline laparotomy, catheters were implanted as reported previously (14). In brief, 7 catheters $(80 \mathrm{~cm}$ long, ID $1.0 \mathrm{~mm}$, OD $1.8 \mathrm{~mm}$; Tygon, Westvaco, Cleveland, $\mathrm{OH}$, USA) were inserted. Catheters for blood sampling were placed in the abdominal aorta, the portall vein and the hepatic vein for metabolic measurements across the portal drained viscera and the splanchnic organs, respectively. Catheters were inserted in the splenic vein and the abdominal aorta for the infusion of para-aminohippuric acid $(\mathrm{PAH})$ to determine plasma flow. For infusion of isotopes and endotoxin, a catheter was implanted in the caval vein above the right renal vein. $A$ thermodilution catheter $(7 \mathrm{~F}$, Baxter Edwards Critical Care, Irvine, CA, USA) was inserted via the femoral vein into the pulmonary artery to monitor cardiac output and pulmonary pressures. In addition, a gastrostomy catheter (I.D. $1.6 \mathrm{~mm}$, O.D. $4.8 \mathrm{~mm}$, Tygon) was placed. All catheters were tunneled through the abdominal wall and skin and sutured to the skin using Vicryl 3-0. To avoid catheter-tip infections and to maintain patency, the catheters were filled with a mixture of $20 \mathrm{mg} / \mathrm{ml}$ gentamycin (A.U.V., Cuyk, the Netherlands) and $225 \mathrm{U} / \mathrm{ml}$ chymotrypsin (Merck, Darmstadt, Germany) in $0.9 \%$ saline. Finally, a jejunostomy was made by transecting the jejunum $20-25 \mathrm{~cm}$ distal to the ligament of Treitz and making an end-to-side anastomosis on the jejunum $10 \mathrm{~cm}$ from the transected end using Prolene 4.0 suture. The transected end was then tunneled through the abdominal wall and skin and sutured to the skin using Prolene 3.0.

\section{Postoperative care}

Postoperative care was standardised as previously described (14). A canvas harness was fitted to each pig to protect the catheters and to allow easy handling of the animal. From the third day onwards, the pigs were fed $1 \mathrm{~kg}$ of food daily. During 
the whole recovery period ( 14 days), the animals remained healthy without signs of infection. In a pilot study four weeks after the operation, the position of the catheter tips was checked under anaesthesia using fluoroscopy.

\section{Experimental protocol}

The animals underwent the experiment (Figure 1) 14 days after surgery. After an overnight fast, the pigs were placed in a movable cage. After taking initial blood samples, all pigs received $3 \mu \mathrm{g} / \mathrm{kg} / \mathrm{h}$ lipopolysaccharide (LPS) from Escherichia coli (055:B5, Sigma Chemicals Co., St. Louis, USA) for 24 hrs via the inferior caval vein catheter. The endotoxin dose used makes this model a zero mortality model (13). Starting 4hrs after the start of the endotoxin infusion and ending $24 \mathrm{hrs}$ after discontinuing the endotoxin infusion, the pigs received either $(\mathrm{n}=6) 10 \mathrm{mg} /$ $\mathrm{kg} / \mathrm{h}$ of the selective NOS2 inhibitor amino-ethyl-isothiourea (AE-ITU, $\mathrm{pH} \mathrm{7.3;}$ Sigma Chemicals $\mathrm{Co}$.) or $(n=7) 0.9 \%$ saline intravenously in a randomised, but non-blinded fashion. All pigs received $0.9 \%$ saline as a fluid support: $30 \mathrm{ml} / \mathrm{kg} / \mathrm{h}$ during the first $8 \mathrm{hrs}$ and $20 \mathrm{ml} / \mathrm{kg} / \mathrm{h}$ during the next $16 \mathrm{hrs}$. During the experimental period, the pigs were kept fasting.

At the end of each experiment, the pig was killed with a lethal dose of thiopental (Nesdonal, Rhône-Poulenc Pharma, Amstelveen, The Netherlands).

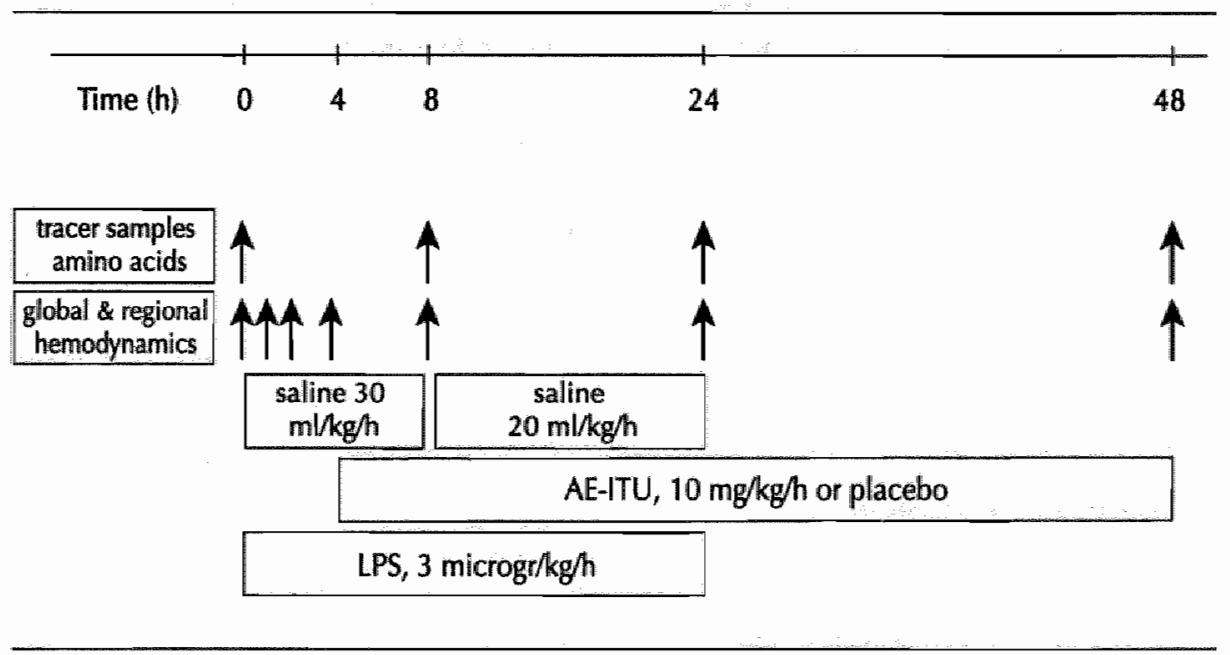

LPS, lipopolysaccharide tendotoxin); AE-ITU, amino-ethyl-isothiouriea

Figure 1 Illustration of experimental design 


\section{Infusion and sampling protocol}

\section{Stable isotopes}

Before the start of the isotope infusions, background blood samples were taken. Two hours after the start of the primed constant infusion of the isotopes through the inferior caval vein catheter, steady state conditions were obtained $(15,16)$. Therefore, blood was sampled $2 \mathrm{hrs}$ after the start of the primed constant infusion of the isotopes. Infusion tracer doses were based on the whole-body tracee appearance (17). L-Iguanidino- $\left.{ }^{15} \mathrm{~N}_{2}\right]$ arginine $\left(\mathrm{N}_{2}\right.$-arginine: infusion: $3.5 \mu \mathrm{mol} / \mathrm{kg} /$ h, prime: $3.2 \mu \mathrm{mol} / \mathrm{kg})$, and $\mathrm{L}$-[ureido- ${ }^{13} \mathrm{C} ; 5,5-{ }^{2} \mathrm{H}_{2}$ ]citrulline $\left(\mathrm{C}_{1}-\mathrm{D}_{2}\right.$-citrulline: infusion: $0.3 \mu \mathrm{mol} / \mathrm{kg} / \mathrm{h}$, prime: $1.5 \mu \mathrm{mo} / \mathrm{kg}$ ) were used (Mass Trace, Woburn, USA). Isotopes were primed-infused, 2 hrs prior to blood sampling $0,8,24$, and $48 \mathrm{hrs}$ after start of endotoxin infusion. Blood was sampled from catheters in the abdominal aorta, the portal vein, and the hepatic vein before isotope infusions were stopped.

\section{PAH}

One hour after the start of the primed-constant PAH (25 mM, A 1422, Sigma Chemicals $\mathrm{CO}$ ) infusion, steady-state conditions for PAH were obtained (14). Therefore, blood was sampled at least $1 \mathrm{~h}$ after the start of the primed-constant infusion of PAH. After an initial bolus of $5 \mathrm{ml}, \mathrm{PAH}$ was infused at a rate of $40 \mathrm{ml} / \mathrm{h}$ through the splenic vein and the aorta catheters.

Meanwhile, a tonometry catheter (14 F, Datex Ohmeda, Finland) was inserted through the jejunostomy for at least $25 \mathrm{~cm}$. A volume of $4.5 \mathrm{ml}$ of $0.9 \%$ saline was instilled into the balloon of the catheter and was allowed to equilibrate for at least $30 \mathrm{~min}$. After equilibration and just prior to the endotoxin infusion, the saline was withdrawn from the balloon and the first $1.5 \mathrm{ml}$ was discarded. The tonometrically-determined $\mathrm{pCO}_{2}\left(\mathrm{PrCO}_{2}\right)$ of the saline was measured simultaneously with the blood gasses. Tonometric measurements were repeated at $0,1,2,4,5,6,8,24$, and $48 \mathrm{hrs}$ after the start of the endotoxin infusion.

Prior to and at $0,4,8,24$, and $48 \mathrm{hrs}$ after starting the endotoxin infusion, triplicate boluses of $5 \mathrm{ml}$ ice-cold saline were injected to estimate the cardiac output with the thermodilution technique, using a cardiac output computer $(9520 \mathrm{~A}$, Baxter Edwards Critical Care, Irvine, CA, USA). At these time points, the mean systemic (MAP) and mean pulmonary arterial pressure (MPAP) were measured in triplicate after zeroing all pressure transducers to the level of the heart. The mean pressures are defined as the (diastolic pressure $\times 2+$ systolic pressure) $/ 3$.

\section{Sample processing}

Promptly after sampling, blood was distributed into pre-chilled, heparinised tubes (Sarstedt, Nümbrecht, Germany) on ice. Hematocrit was determined to calculate plasma flow from blood flow. For blood gas analysis (arterial pH, $\mathrm{pO}_{2}, \mathrm{pCO}_{2}, \mathrm{HCO}_{3}$-, and base excess), $0.2 \mathrm{ml}$ blood was sealed airtight in heparinized $1 \mathrm{ml}$ syringes and 
immediately analysed on an automatic blood gas system (Acid Base Laboratory (ABL3), Radiometer, Copenhagen, Denmark) and corrected for pig haemoglobin and core temperature of the animal. The core temperature of the pig was measured in the central venous blood using the pulmonary antery floatation catheter.

For PAH determination, $300 \mu$ of whole blood were added to $600 \mu /$ of $12 \%(\mathrm{~W} / \mathrm{v})$ trichloroacetic acid, mixed thoroughly, and centrifuged $\left(4^{\circ} \mathrm{C}\right.$ for $5 \mathrm{~min}$ at $\left.8,500 \mathrm{~g}\right)$. For glucose and lactate determinations, $900 \mu$ plasma were added to $90 \mathrm{ml} 50 \%$ trichloroacetic acid solution. For analysis of amino-acid concentrations, plasma was deproteinized by mixing $500 \mu l$ with $20 \mu \mathrm{g}$ dry sulfosalicylic acid. All samples were frozen in liquid nitrogen and stored at $-80^{\circ} \mathrm{C}$ until further analysis.

\section{Biochemical analysis}

PAH was detected spectrophotometrically after deacetylation of the supernatant at $100^{\circ} \mathrm{C}$ for $45 \mathrm{~min}(14)$. Plasma glucose, pyruvate and lactate concentrations were determined by automated HPLC (Pharmacia, Woerden, the Netherlands). Concentrations and enrichments of amino acids were calculated as tracer-to-tracee ratios (TTR) and were determined by a fully automated liquid chromatography-mass spectrometry system (LC-MS, Thermoquest LCQ, Veenendaal, The Netherlands) after pre-column derivatisation with o-phthaldialdehyde (18).

\section{Calculations}

Plasma flow ( $\mathrm{ml} / \mathrm{kg}$ bw/min) was calculated using PAH as an indicator for the dye-dilution technique (14). Regional oxygen delivery and consumption were calculated as the product of regional blood flow, and the arterial and venous oxygen content, respectively. The systemic oxygen delivery was calculated as a product of cardiac index, as measured by thermodilution technique, and arterial oxygen content. Cardiac index is defined as the cardiac output per body weight.

The whole body (WB) production of arginine and citrulline were calculated using formula (1), in which I represents the rate of the tracer infused and $T T R_{A^{\prime}}$ the tracer-to-tracee ratio of $N_{2}$-arginine or $C_{1}-D_{2}$-Citrulline in the arterial plasma (19)

(1) $W B=1 / T T R_{A A}$

Formula (2) was used to calculate the rate of conversion of $N_{2}$-arginine into $N_{1}$-citrulline, representing the whole-body NO synthesis production.

(2) $\mathrm{WB}_{\mathrm{ARG}(2) \mathrm{CIT(1)}}=\mathrm{Q}_{\mathrm{CIT}(3)} \times\left(\mathrm{TTR}_{\mathrm{AACIT}(1)} / \operatorname{TTR}_{\mathrm{AA} \text { ARG(2) }}\right)$

Whole-body de novo synthesis of arginine was calculated using formula (3):

(3) $W_{\text {CIT(3) ARG(3) }}=Q_{A R G} \times\left(T T R_{A A A R G(3)} / T T R_{A A C I T(3)}\right)$ 
The rate of NO synthesis by individual organs ( $\mathrm{Syn} \mathrm{NO}$ ) can be calculated from conversion of $\mathrm{N}_{2}$-arginine to $\mathrm{N}_{1}$-citrulline across that organ. The term $\mathrm{nb} \mathrm{N}_{\mathrm{NI}-\mathrm{CIT}}$ represents the tracer net balance of the $N_{1}$-citrulline isotope in arterial and venous plasma, respectively. Because of possible fractional extraction of citrulline and, therefore, $\mathrm{N}_{1}$-citrulline $\left(\mathrm{FE}_{\mathrm{CIT}}\right)$ by the organ, $\mathrm{nb} \mathrm{N}_{\mathrm{N}-\mathrm{CIT}}$ must be corrected for the loss of $N_{1}$-citrulline tracer across the organ, which is possible by using the $C_{1} D_{2}$-citrulline isotope in the equation (4). The term (1 - FE) represents the fraction of total amino acid that bypasses metabolism in the organ and so appears in the output and is used for correction of the $\mathrm{N}_{1}$-citrulline $\mathrm{nb}$ (5). Organ $\mathrm{Syn}_{\mathrm{NO}}$ is derived from formula (6), in which $T T R_{A A}$ N2-ARG represents the arterial $N_{2}$-arginine enrichment. The TTR of $\mathrm{N}_{2}$-arginine in the arterial plasma free pool was assumed to be the same as the TTR of $\mathrm{N}_{2}$-arginine in the direct precursor pool of $\mathrm{NO}$ synthesis.

(4) $\left.\mathrm{FE}_{\mathrm{CIT}}=\left(T T R_{\mathrm{AA}} \times[\mathrm{A}]-T \mathrm{TR} \mathrm{R}_{\mathrm{V}} \times[\mathrm{V}]\right) / T \mathrm{TR}_{\mathrm{AA}} \times[\mathrm{A}]\right)_{\mathrm{CID}} \mathrm{ClT}$

(5) $\mathrm{nb}_{\mathrm{N1} 1-\mathrm{CIT}}=$ flow $\times\left\{[\mathrm{V}] \times T T R_{\mathrm{V}}-[\mathrm{A}] \times T \mathrm{TR}_{\mathrm{AA}} \times\left(1-\mathrm{FE}_{\mathrm{CIT}}\right)\right\}_{\mathrm{N} 1-\mathrm{CIT}}$

(6) $\mathrm{Syn}_{\mathrm{NO}}=\mathrm{nb}_{\mathrm{N} 1-\mathrm{CIT}} / \mathrm{TT} \mathrm{R}_{\mathrm{AAN} 2-\mathrm{ARG}}$

\section{Statistics}

Results are presented as means \pm SD. Levels of significance were set at $p<0.05$. The data were subjected to multiple-way ANOVA analysis to test the effect of time and to compare group differences during the $48 \mathrm{hrs}$ study period, using time as a within factor time with 4 levels (repeated measures: $t=0,8,24$, and $48 \mathrm{~h}$ ) and as between-factors treatment ( $\mathrm{AE}-\mathrm{ITU}$ or placebo). Error alpha values are reported in tables and figures in which $\mathrm{P}_{\mathrm{T}}$ indicates the significance of repeated measures over time, $P_{T \times C}$ the interaction of repeated measures and treatment group (time $x$ group) and $P_{G}$ the effect of AE-ITU treatment. Survival was compared using a log rank test and the Kaplan-Meier estimate for survival.

\section{RESULTS}

\section{Animals}

In the control animals $(n=7)$ body weight rose from $22.2 \pm 0.9$ to $26.4 \pm 1.4$ $\mathrm{kg}$ during the first $24 \mathrm{~h}$ of the experiment and declined after the cessation of the endotoxin infusion to $25.0 \pm 1.5 \mathrm{~kg}$. These changes in body weight were not significantly different from the changes in body weight of the AE-ITU-treated animals $(n=6)$ : an increase from $21.0 \pm 0.6$ to $28.3 \pm 1.4 \mathrm{~kg}$ at $24 \mathrm{hrs}$ and a decline to $24.4 \pm 1.0 \mathrm{~kg}$ at $48 \mathrm{~h}\left(\mathrm{P}_{\mathrm{G}}=1.0, \mathrm{P}_{\mathrm{T}}<0.0001, \mathrm{P}_{\mathrm{T} \times \mathrm{C}}=0.4\right)$. 

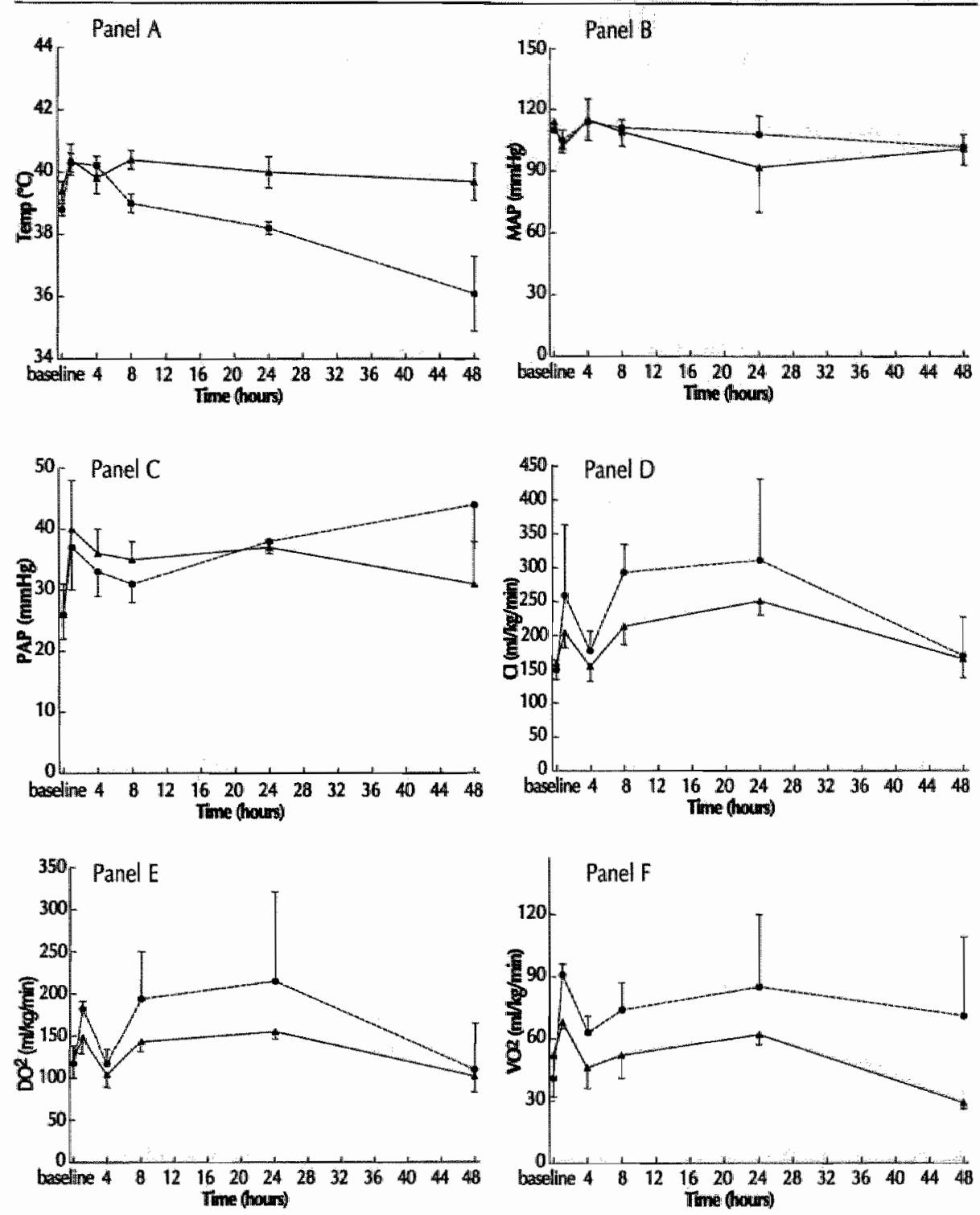

Data are presented as mean \pm standard error. Panel A: change in cone temperature in control (solid lines) and AE-rTU treated (dotted lines) animalsus Statistics by multiple-way ANOVA lsee statistics): $P_{C}<0.0001, P_{Y}<0.0001, P_{T \times G}<0.0001$.

Panel $B$ : change in mean arterial pressure (MAP) in control and $A E-$ ITU treated animals; $P_{G}=0.8, P_{T}=0.6, P_{T X G}=0.8$.

Panel $C$ : change in pulmonary arterial pressure $(\mathrm{PAP}) ; \mathrm{P}_{\mathrm{G}}=0.6, \mathrm{P}_{\mathrm{T}}=0.04, \mathrm{P}_{\mathrm{TXG}}=1.0$.

Panel $D$ : change in cardiac index $(C)$ in control and AE-lTU treated animals; $P_{C O}=0.06, P_{T}=0.06, P_{T \times C}=1.0$.

Panel $E$ : change in global oxygen delivery $\left(\mathrm{DO}_{2}\right)$ in control and $A E-t T U$ treated animals; $P_{C}=0.1, P_{T}=0.04, P_{T \times C}=0.6$.

Panel F: change in global oxygen consumption $\left(\mathrm{NO}_{2}\right)$ in control and $\mathrm{AE}-\mathrm{TT}$ treated animals $\mathrm{P}_{\mathrm{C}}=0.09_{n} \mathrm{P}_{\mathrm{T}}=0.5, \mathrm{P}_{\mathrm{TKC}}=0.6$

Figure 2 Effects of AE-ITU treatment on core temperature and global haemodynamic parameters 
Compared to their own baseline values, the temperature of endotoxin-infused animals was elevated between 4 and $24 \mathrm{hrs.}$. The infusion of AE-ITU significantly decreased the core temperature between 8 and $48 \mathrm{hrs}$ compared to control animals $\left(\mathrm{P}_{\mathrm{TxC}}<0.0001\right)$. At $48 \mathrm{hrs}$, the core temperature in AE-ITU-treated animals (36.1 \pm $1.2^{\circ} \mathrm{C}$ ) was lower than before the start of the experiment (Figure 2, panel A).

\section{Survival}

None of the animals in the control group died in this 'zero-mortality' model of porcine endotoxaemia. However, in the AE-ITU group $50 \%$ of the animals died between 12 and $24 \mathrm{hrs}$ after stopping the endotoxin infusion (Figure $3 ; p=0.04$ on log-rank analysis). Clinically, these pigs suffered from respiratory distress, had a cold and clammy skin, were oliguric or anuric, and had severe generalised edema (weight at $48 \mathrm{hrs}$ in non-survivors is $26.8(2.2)$ versus $23.8(2.0)$.

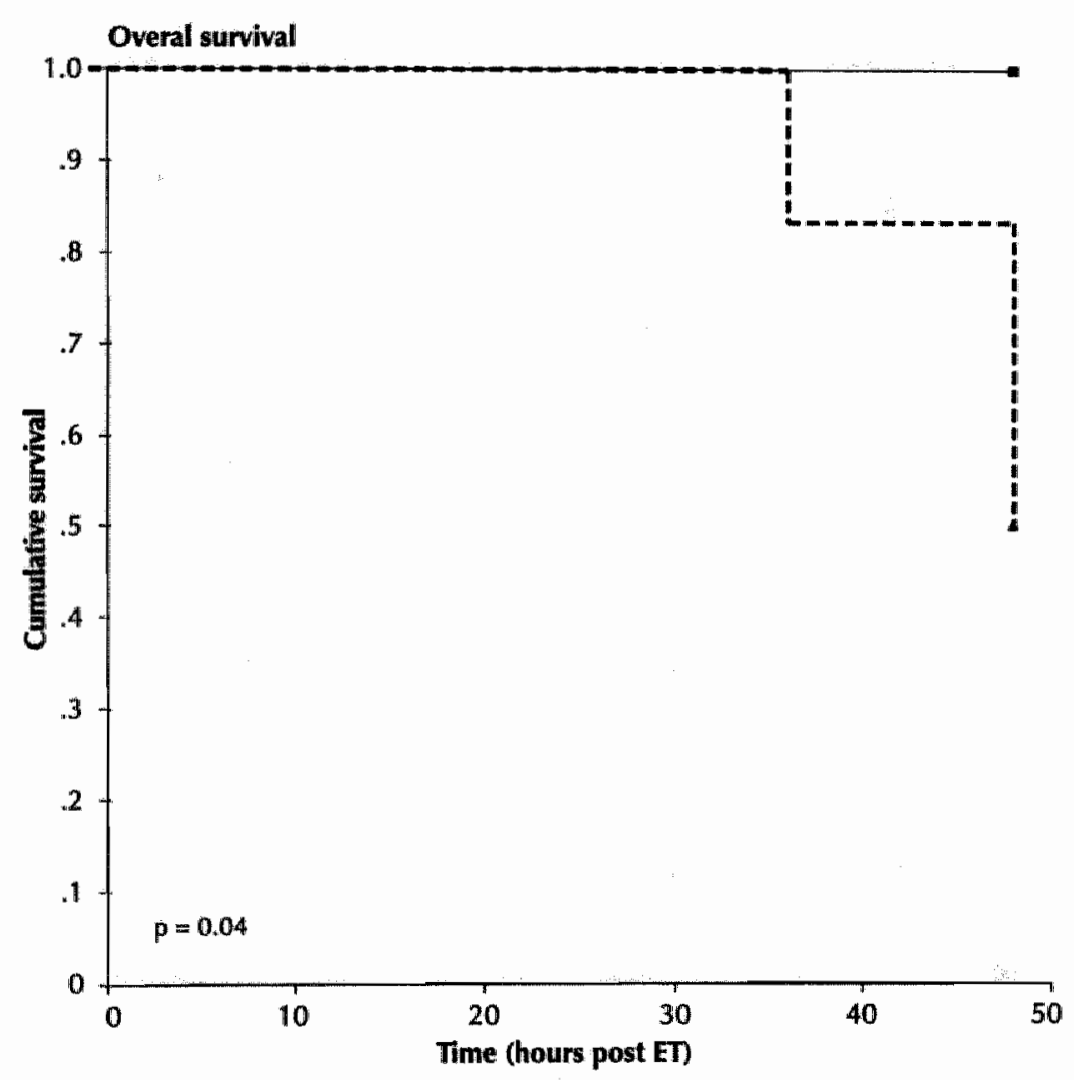

Overall survival curves during $\mathrm{AE}-1 \mathrm{TU}$ (dotted line) and control (solid line) treatment. Log rank analysis showed significant difference in mortality $(p=0.04)$.

Figure 3 Cumulative survival curves during AE-ITU treatment during porcine endotoxaemia 

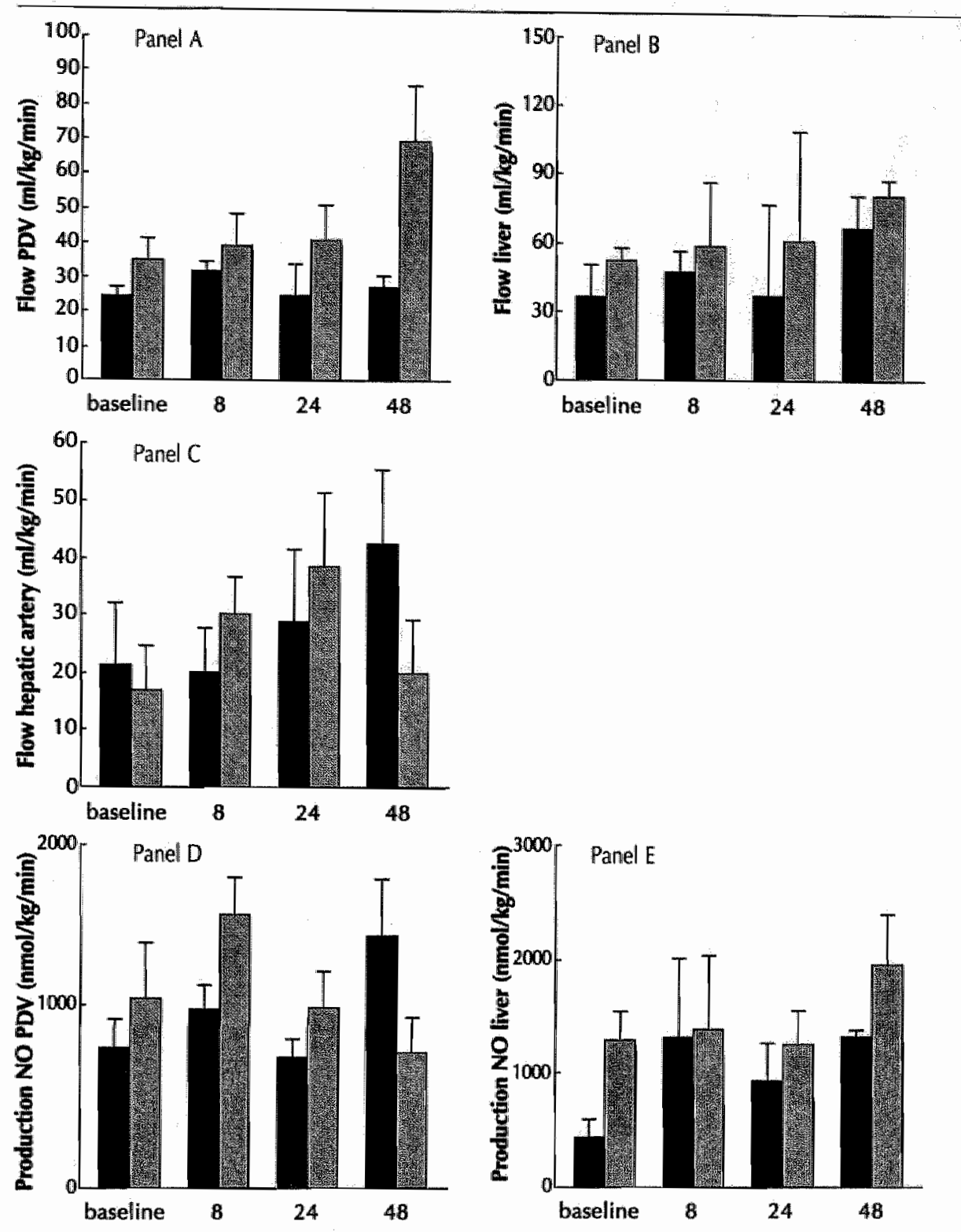

Data are presented as mean \pm standard error. Panel $A$ : change in portal-drained viscera flow (PDV) flow in control (solid bars) and AE-TTU supplemented (shaded bars) animals; statistics by multiple way ANOVA; $\mathrm{P}_{\mathrm{G}}=0.006, \mathrm{P}_{\mathrm{T}}=0.2, \mathrm{P}_{\mathrm{XXG}}=0.046$. Panel $B$ : change in liver flow in control and AE-ITU treated animals; $; P_{C_{i}}=0.3, P_{T}=0.7, P_{T \times G}=0.7$. Panel $C:$ change in hepatic artery flow in control and AE-ITU treated animals; $P_{C}=0.8_{n} P_{T}=0.8_{r}, P_{T X C}=0.7$. Panel $D$ : change in: PDV NO flux in control and AE-TTU treated animals; $P_{G}=0.5, P_{T}=0.07, P_{T W G}=0.05$. Panel E: change in liver NO flux in control and AE-TTu treated animals: $P_{G}=0.2, P_{Y}=0.001, P_{T K C}=0.8$. 


\section{Global haemodynamics}

The changes in MAP upon LPS infusion were not significantly different from baseline at 4, 8, 24, and $48 \mathrm{hrs}$ in control and AE-ITU animals (Figure 2, panel B). In contrast, the MPAP increased significantly over time during the first $24 \mathrm{hrs}$, but this increase was similar in control and AE-ITU animals $\left(\mathrm{P}_{\mathrm{T} \times \mathrm{C}}=1.0\right.$ ) (Figure 2, panel $\mathrm{C}$ ). CardiaC index tended to increase over time and to be higher in AE-ITU than in control animals $\left(P_{G}\right.$ and $P_{T}=0.06$ ) (Figure 2, panel D). Global oxygen delivery increased significantly $\left(\mathrm{P}_{\mathrm{T}}=0.04\right)$ over time. The global $\mathrm{DO}$ and $\mathrm{VO}_{2}$ were not different between the two treatment groups, although the average $D O_{2}$ was 2 -fold higher in AE-ITU-treated animals than in controls (Figure 2, panels $E$ and $F$ ).

\section{Regional haemodynamics and tonometry}

During endotoxin treatment, blood flow through the PDV temporarily increased in control animals, whereas that through the hepatic artery remained unchanged (Figure 4, panels $A$ and $C$ ). As a result, total blood flow through the liver did not change (Figure 4, panel B). Oxygen delivery, consumption, extraction and saturation in PDV and liver also did not change in control animals (Table 1). When the animals were additionally treated with AE-ITU, blood flow through the PDV increased significantly $\left(P_{T_{x C}}<0.05\right)$, and that through the hepatic artery gradually increased to reach a more than 2-fold higher vallue after 24 hrs.

Oxygen consumption, extraction and saturation in PDV were not affected by AE-ITU treatment, but oxygen consumption and extraction in the liver increased $1.7-2$-fold $\left(P_{\mathrm{TxG}}=0.01\right.$ and 0.005 , respectively) (Tabel 1). However, oxygen delivery to the liver decreased after $24 \mathrm{hrs}$. During the $24 \mathrm{hrs}$ following cessation of endotoxin infusion, blood flow in the hepatic artery increased $50 \%$, causing an increase in total liver blood flow in the control animals. Oxygen consumption in the PDV declined to $50 \%$, whereas oxygen extraction increased $50 \%$. Changes were even more dramatic in AE-ITU-treated animals. The blood flow through the PDV increased $70 \%$, whereas that through the hepatic artery declined to $50 \%$. As a result, total blood flow through the liver did not change much, but oxygen delivery decreased to $50 \%$ and oxygen extraction rose by $50 \%$. As a result, oxygen saturation in the hepatic wein declined to $50 \%\left(\mathrm{P}_{\mathrm{T} \times \mathrm{G}}=0.03\right)$.

The LPS infusion increased the intramucosal $\mathrm{PrCO}_{2}$ significantly in both treatment groups (Figure 5). When the animals were additionally treated with AE-ITU, $\mathrm{PrCO}_{2}$ decreased from 4 hrs until it reached baseline values. This difference between control and AE-ITU treated animals was not longer present after 24 hrs LPS infusion.

\section{In vivo NO production and amino acid levels}

During 24 hrs of endotoxin treatment, whole-body arginine appearance and de nowo synthesis increased significantly, the adaptive response becoming detectable 


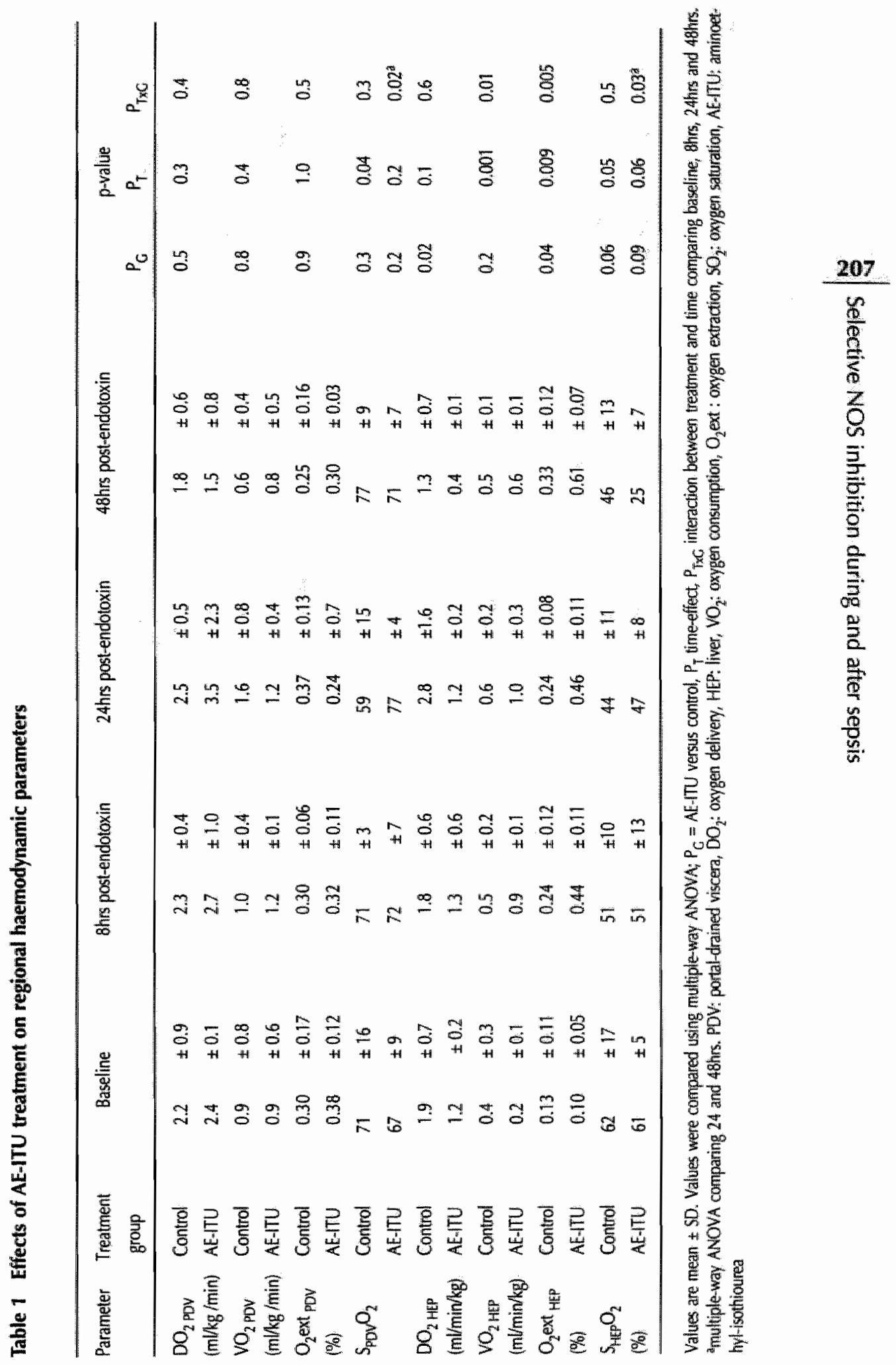




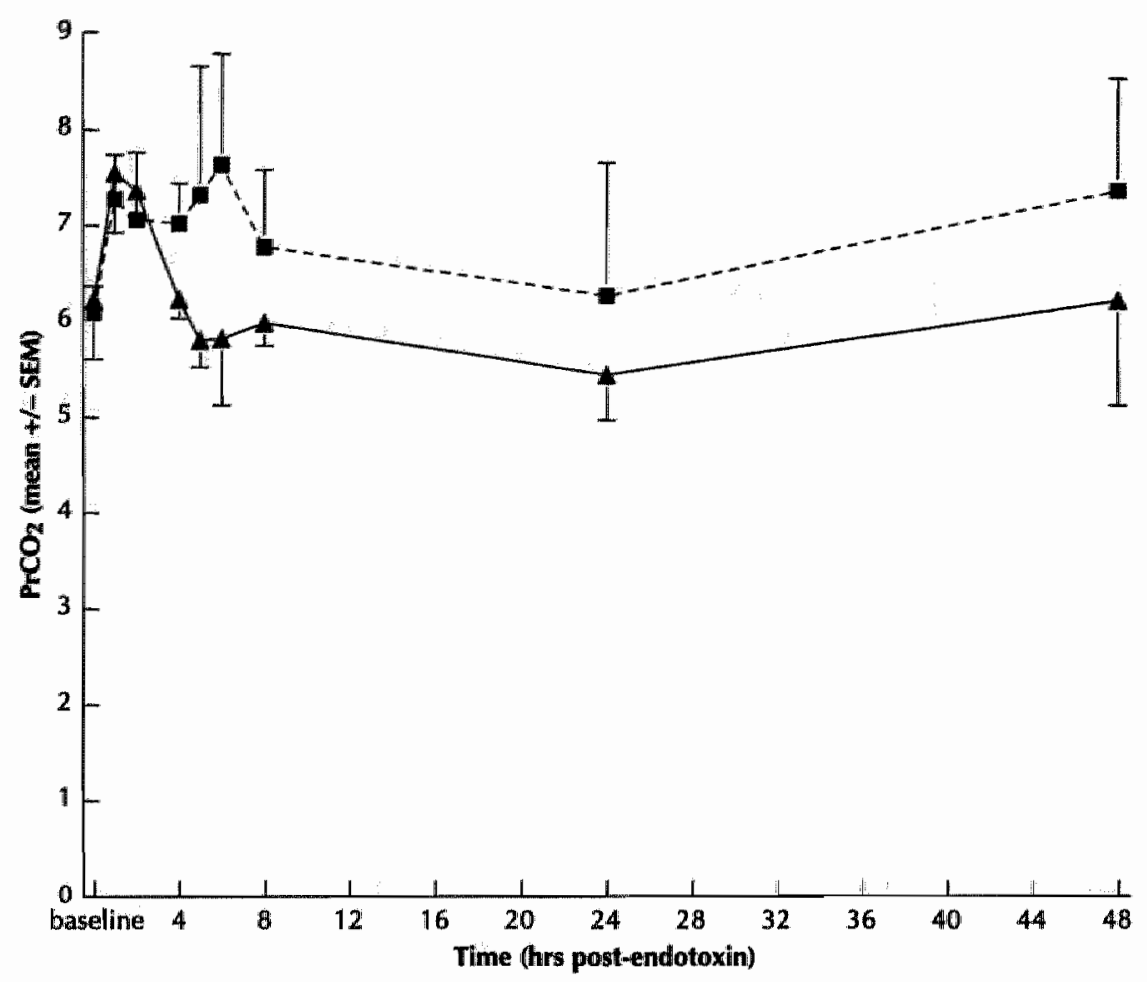

Data are presented as mean \pm standard error. Treatment of AE.ITU (solid line) induces a rapid decrease $(p=0.01$ ) in intramucosal $\mathrm{PrCO}_{2}$ compared to control (dotted line) during endotoxaemia. Differences between groups were not significantly different alter $24 \mathrm{hrs}$.

Figure 5 Effect of AE-ITU treatment on jejunal intramucosal $\mathrm{PrCO}_{2}$ during porcine endotoxaemia

after 8 hrs of treatment (Fig. 6, panels $C$ and D). The adaptive response of arginine synthesis was not reflected in either an enhanced precursor (citrulline) or product (NO) synthesis (Fig. 6, panels A and B). After discontinuing endotoxin treatment, whole-body arginine appearance returned to normal within $24 \mathrm{hrs}$, but de novo arginine biosynthesis remained elevated. If AE-ITU was additionally infused, the adaptive response of arginine appearance was suppressed to below basall levels (Fig. 6, panels C and D). Similarly, whole-body citrulline appearance and, more pronounced, NO synthesis were suppressed (Fig. 6, panels A and B).

The NO synthesis in the PDV in control animals tended to increase during endotoxaemia, while increased significantly in the liver $(p=0.07$ and $p=0.001)$. AE-ITU did not change the NO synthesis in the liver during the experiment $(p=0.8)$, while tended to decrease PDV NO synthesis $(p=0.05)$.

The arterial levels of glucose tended to decline during endotoxin treatment, 

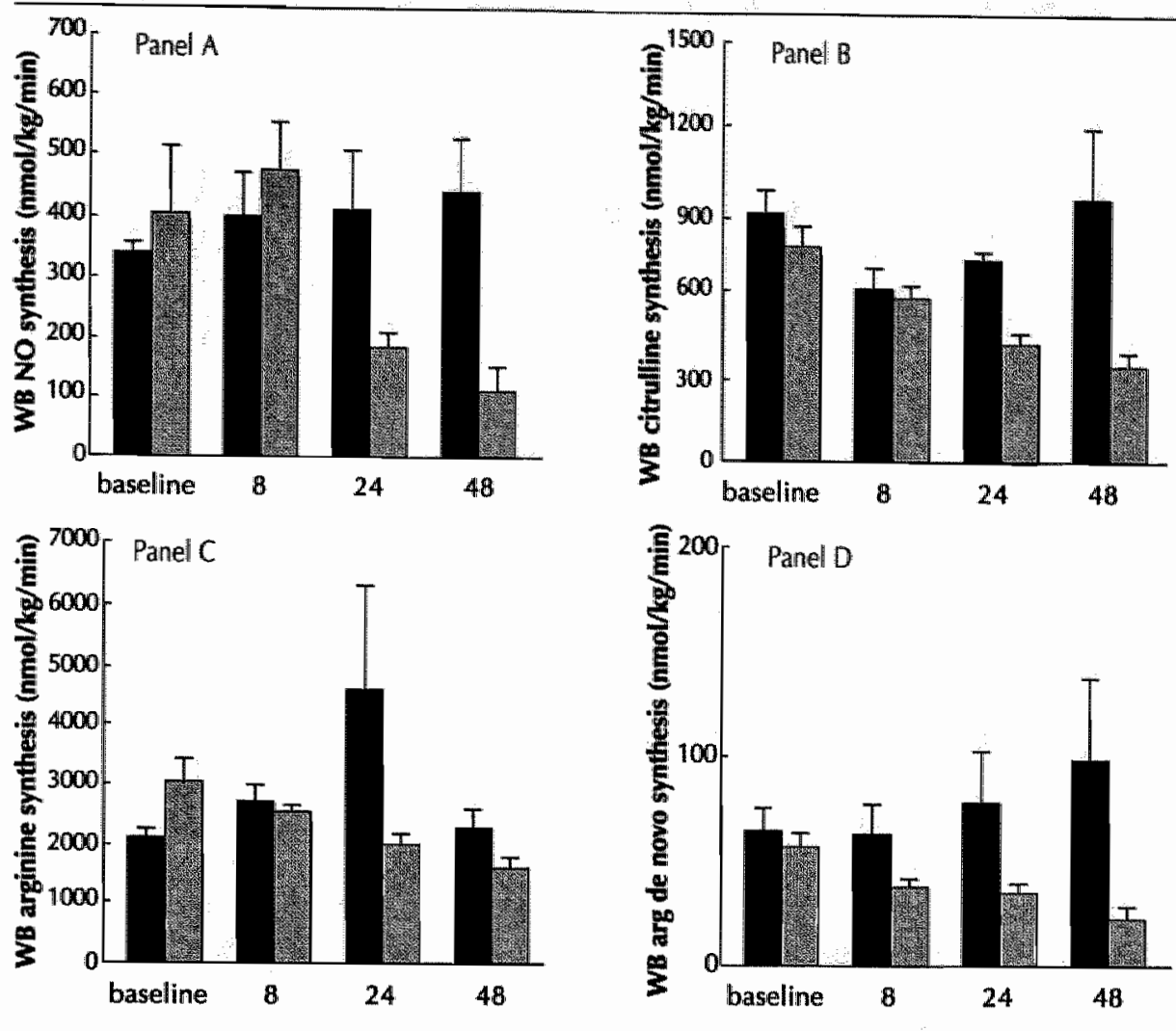

Data are presemted as mean \pm standard error. Panel A: change in whole-body (WB) NO synthesis in control (solid bars) and AE-ITU supplemented (shaded bars) animals; statistics by multiple way $A N O V A ; P_{G}=0.05, P_{T}=0.1, P_{T \times G}=0.02$,

Panel $B$ : change in WB citwiline appearance in control and AE- $17 \mathrm{U}$ treated animals" $P_{\mathrm{G}}<0.0001, \mathrm{P}_{\pi}<0.0001, \mathrm{P}_{\mathrm{TxG}}<0.0001$. Panel $C$ : change in $W B$ arginine appearance in control and $A E-1 T U$ treated animals $S_{i}=0.07, P_{T}=0.2, P_{T \times G}=0.007$.

Panel D: change in WB arginine de nowo synthesis in control and $A E-1 T U$ treated animats; $P_{G}<0.0007, P_{T}=0,3, P_{T \times G}=0.03$.

Figure 6 Whole-body arginine-NO metabolism during AE-ITU and saline (controls) treatment of hyperdynamic endotoxaemia

without additional effect of AE-ITU treatment. The arterial concentrations of lactate and pyruvate increased significantly less in AE-ITU-treated animals than in controls (both: $p=0.003$ ) (Table 2). However, since lactate concentrations rose more than pyruvate concentrations, a distinct and similar rise in lactate-pyruvate ratio was found during endotoxin infusion in both control and AE-ITU-treated pigs. After cessation of endotoxin treatment, lactate and pyruvate concentrations dropped in control animals, but because lactate llevels continued to rise, whereas pyruvate levels remained low in AE-ITU-treated pigs, an extremely high lactate-pyruwate ratio developed in this group $(>20 ; p=0.009)$. 


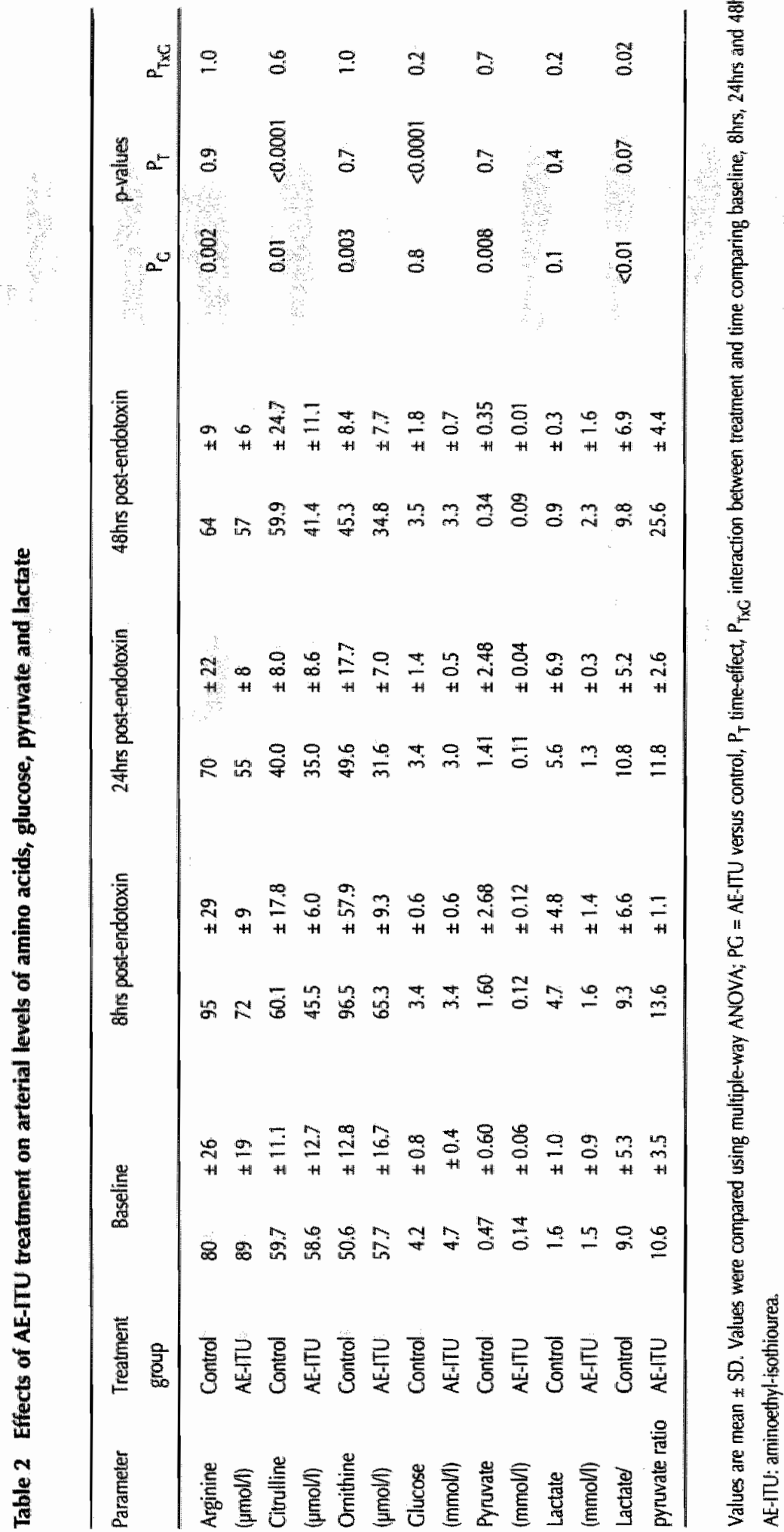

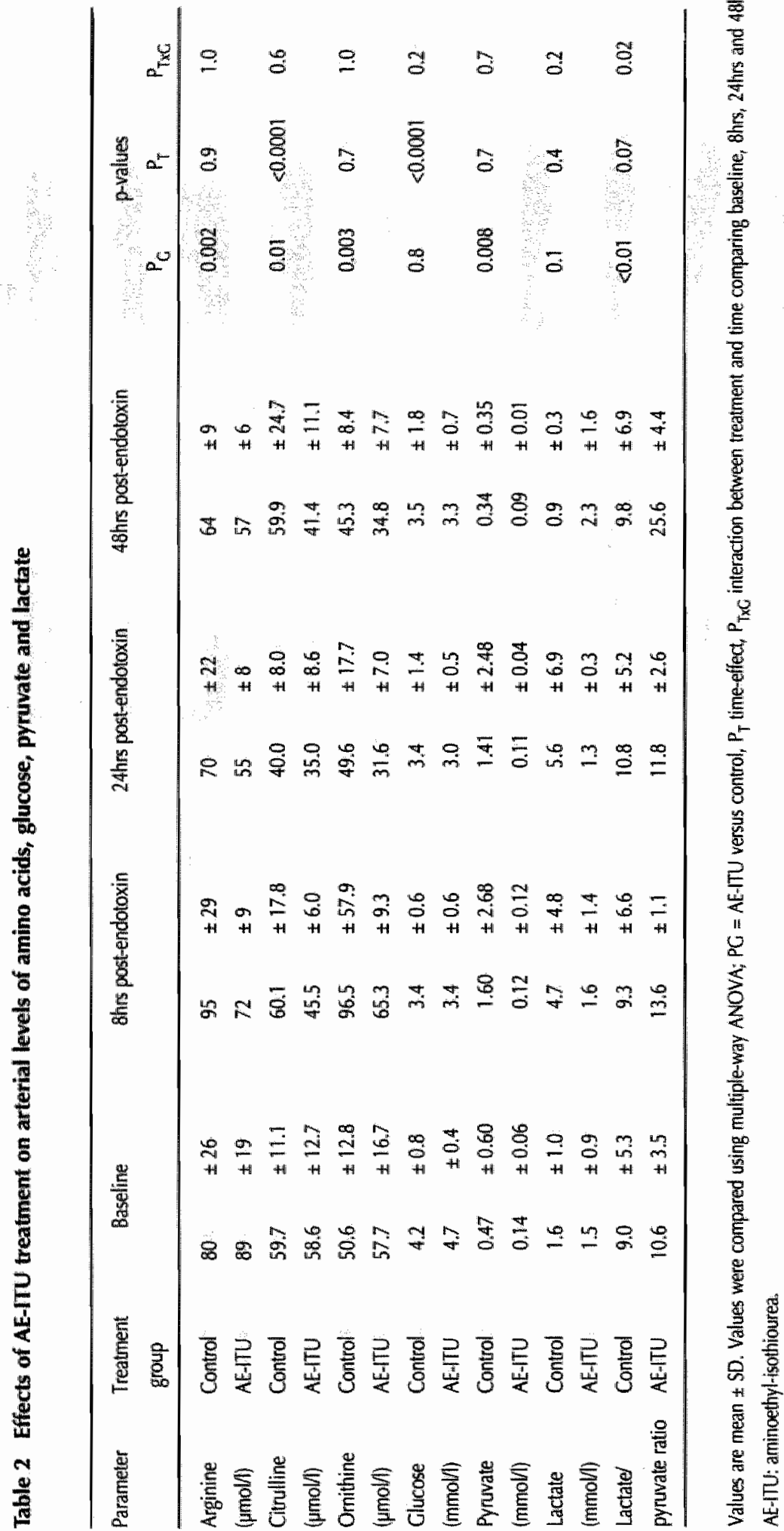

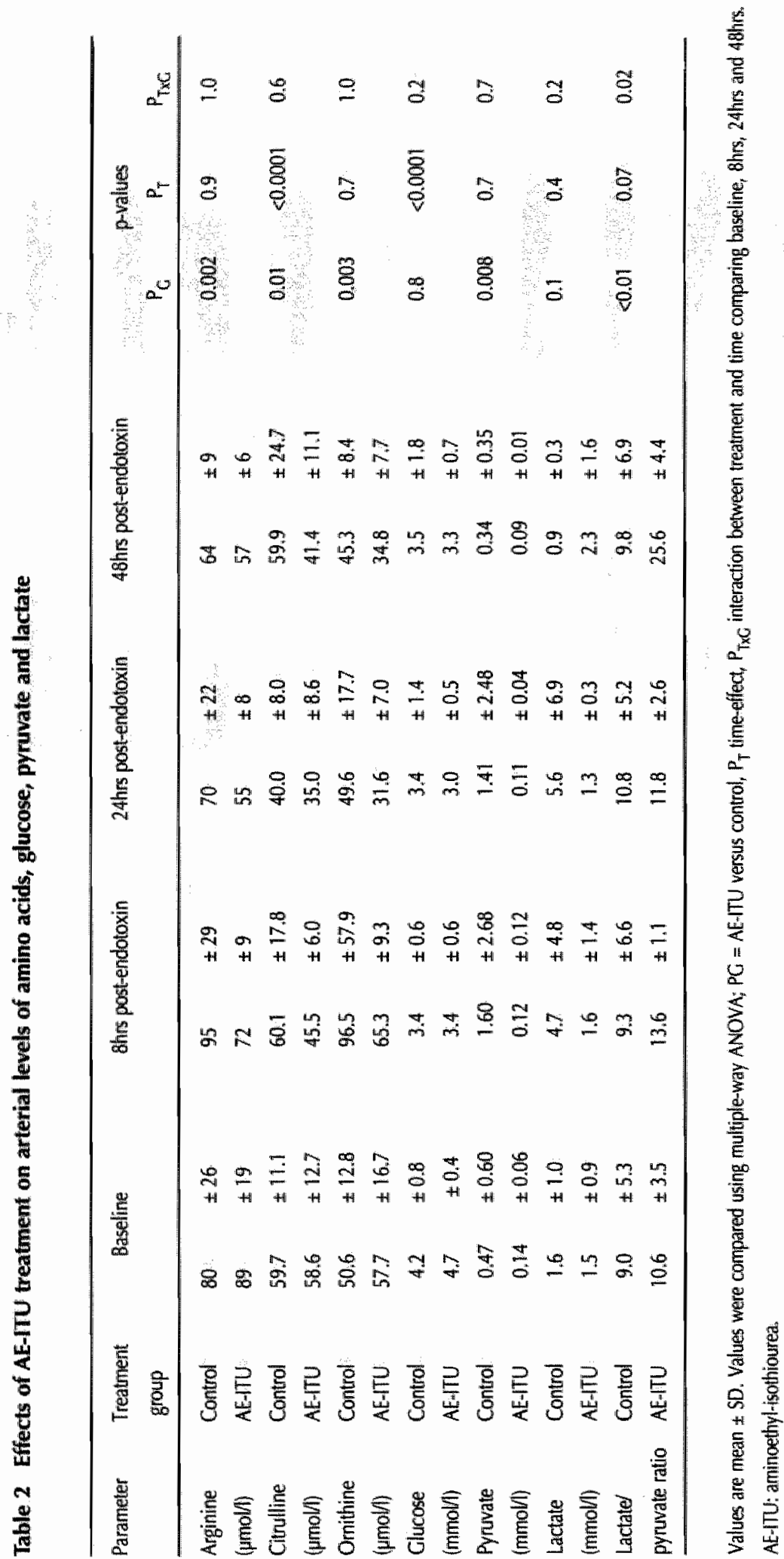

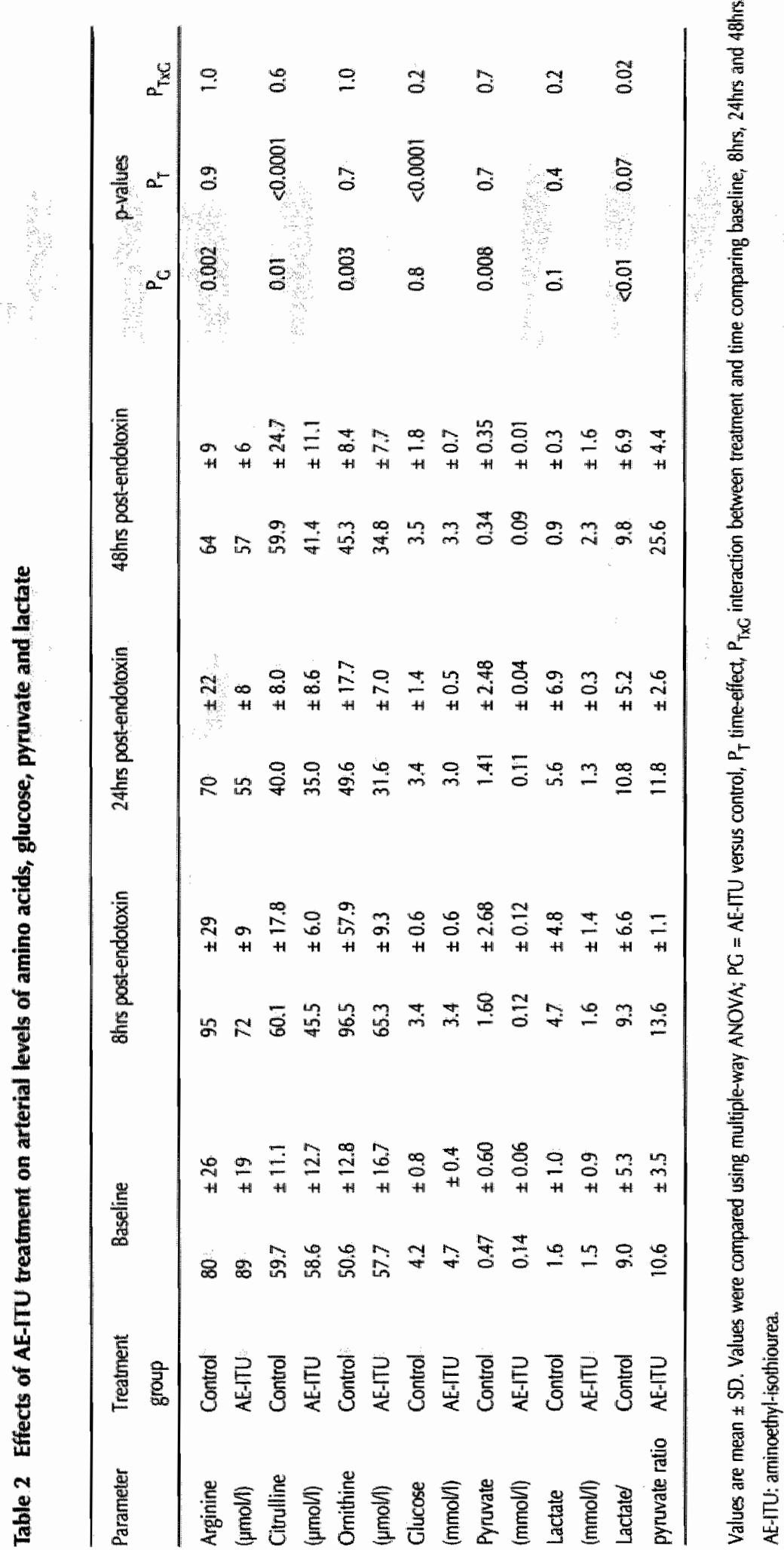




\section{Discussion}

The major conclusion from this study on porcine hyperdynamic endotoxaemia is that selective NOS2 inhibition temporarily improves hepatosplanchnic perfusion and metabolism, but entails detrimental effects on hepatosplanchnic perfusion after cessation of endotoxin infusion, a condition similar to initial recovery in patients. Selective NOS2 inhibition as a treatment for endotoxaemic shock is, therefore, a precarious strategy.

\section{The porcine LPS-infusion model of sepsis}

The choice of the model is important since it may influence the effects of the intervention. Administration of intermediate doses of endotoxin over extended periods, in contrast to a bolus infusion containing a high dose of endotoxin, resembles the prolonged and gradual release of endotoxin into the circulation in the clinical situation. The dose of endotoxin used in our animal model did indeed induce a hyperdynamic circulation (13), but no mortality. Many studies that use larger amounts of endotoxin (20-22) induce high mortality rates within hours after administration, precluding a proper evaluation of the underlying pathophysiology. Some studies (23) used intra-portal infusion of (lower; $1.7 \mu \mathrm{mol} / \mathrm{kg} / \mathrm{h}$ ) endotoxin in order to induce more pronounced responses from the liver. Since the source of endotoxin is not always located in the PDV, we opted for a systemic infusion of endotoxin.

AE-ITU is a highly selective and potent NOS2 inhibitor (24). In our study, infusion of AE-ITU was started $4 \mathrm{hrs}$ after the start of endotoxin infusion, as NOS2 protein does not begin to accumulate until after 2-3hrs of LPS administration (32). In clinical sepsis, treatment will be started after the first symptoms of sepsis have appeared. The dose of AE-ITU used in our study effectively blocks NOS activity, but does not affect NOS1 and NOS3 expression and activity (25). Moreover, AE-ITU only causes a minor inhibition of arginase activity (26). Finally, in vitro and in vivo data $(25,27)$ indicate that the toxic side effects that have been ascribed to some selective NOS2 inhibitors (28), do not apply to AE-ITU.

Whether or not selective inhibition of NOS2 activity with AE-ITU is a beneficial or detrimental adjuvant treatment for endotoxaemia and sepsis was evaluated using a combination of perfusion and metabolic parameters. In this respect it should be kept in mind that increased flow not necessarily implies improved cellular metabolism. In fact, Saetre et al. hypothesised that the primary effect of selective NOS2 inhibition was on metabolism, which in turn affected organ perfusion (25). We therefore measured both parameters simultaneously.

\section{Global perfusion}

Treatment of hyperdynamic endotoxaemia with the specific NOS2 inhibitor AE-ITU did not change global perfusion significantly. Another selective NOS2 inhibitor, 
$1400 \mathrm{~W}$, also did not affect systemic haemodynamic parameters during prolonged hyperdynamic porcine endotoxaemia (29). Provided it is given in a sufficiently high dose, AE-lTU may even prevent the fall in arterial blood pressure and systemic vascular resistance that occurs during the terminal phase of endotoxaemia in the pig (25).

\section{Regional perfusion}

The changes in oxygen extraction suggest that the metabolic demands of the liver of control animals increase during endotoxaemia, whereas those of the intestine do not. In agreement with an earlier suggestion (30), we observed that, compared to controls, AE-ITU treatment significantly increased oxygen extraction and consumption, but not oxygen delivery in the liver. This lack of balance between supply and demand led to a significantly higher increase in the lactate/pyruvate ratio compared to control animals. Other studies have found that AE-ITU treatment did not reduce sepsis-induced systemic acidosis (25). In addition, the deterioration of liver morphology in LPS and AE-ITU-treated animals (11) also suggests a poor perfusion of this organ, possibly due to shunting (31).

After cessation of the endotoxin infusion, flow in the PDV remained similar in the control animals, while flow in the hepatic artery increased. Interestingly, the opposite effect occurred in the AE-ITU treated animals, with a $50 \%$ reduction in flow in the hepatic artery. The increased oxygen extraction by the liver and the decreased oxygen saturation in the hepatic vein indicate that the liver tries to compensate for the decreased oxygen supply.

During selective NOS2 inhibition, intramucosal $\mathrm{PrCO}_{2}$ temporarily decreased in endotoxaemic pigs in both our study and that of Pittner et al. (29). These results seem to reflect improved oxygenation in the splanchnic mucosa during endotoxaemia. Indeed, NOS2 inhibition not only increased global intestinal blood flow, but also perfusion of the intestinal wall and capillary haemoglobin oxygen content (26). Intramucosal $\mathrm{PrCO}_{2}$ is, therefore, a good marker for intestinal tissue oxygenation.

\section{In vivo NO production}

In this study, the whole-body in vivo measurements of NO production were determined using the ${ }^{15} \mathrm{~N}_{2}$-arginine to ${ }^{15} \mathrm{~N}$-citrulline conversion method. Whereas whole-body NO production only tended to increase, NO production in the PDV and liver increased significantly. These data confirm our earlier findings (13). Nitrate and nitrite release into the portal vein, a surrogate marker for NO production in the gut, also increased (29).

AE-ITU treatment causes a significant reduction in whole-body nitric oxide production. In agreement, administration of the selective NOS2 inhibitor $1400 \mathrm{~W}$ reduces exhaled NO after $24 \mathrm{hrs}$ of endotoxin infusion (29). It is, therefore, likely that the haemodynamic and metabolic effects of AE-ITU are at least partly caused by reduction in NO production. Previous studies indicate that during endotoxaemia 
NOS1+3-dependent NO production in the liver is reduced (33). Similarly, injury reduces NOS1+3 and increases NOS2 mRNA concentration in vascular smooth muscle cells of pigs (34).

Although the whole-body rate of arginine appearance increased up to $24 \mathrm{hrs}$ in the control group, systemic arginine levels decreased. The lowering of circulating arginine may be caused by the decreased dietary arginine availability due to the fasted state and by an accelerated arginine turnover due to arginase induction (13). AE-ITU treatment reduced both the rate of appearance of arginine during and after endotoxin infusion, leading to a further reduction in arginine levels relative to control animals. The decrease in the rate of arginine appearance appears to result from a decreased rate of de novo arginine synthesis and perhaps also from a suppression of protein catabolism, although whole-body protein degradation as measured by phenylalanine production was not significantly suppressed by AE-ITU (data not shown).

\section{Survival}

AE-ITU treatment improved survival in a $100 \%$ mortality model (group-A streptococci-induced sepsis), but in this models, animals are only studied for $6 \mathrm{hrs}$ (32).

Until now, however, no studies have been published that addresses the mortality resulting from NOS2 blockade during and after prolonged endotoxin administration. Although our porcine model of L.PS-induced endotoxaemia is normally a zero-mortality model, mortality was significantly increased by AE-ITU treatment. Intriguingly, however, pigs died only after discontinuation of endotoxin infusion and displayed signs of multiple-organ failure. Previously, AE-ITU treatment has been associated with a better survival (32). We can only speculate at present about the reasons why AE-ITU treatment causes death. However, we observed that PDV perfusion continued to increase, while liver perfusion decreased after stopping the LPS infusion. The high PDV perfusion is probably maintained by the persistingly high PDV $\mathrm{O}_{2}$ consumption and is possibly caused by the persistingly high NO production. This finding is reminiscent of the circulatory changes that occur gradually during decompensation of liver cirrhosis 35 ). We, therefore, speculate that the rapid onset of the circulatory changes does not grant the victims sufficient time to adapt.

In conclusion, selective inhibition of NOS2 improves hepatosplanchnic perfusion and metabolism during the initial phase of endotoxaemia. However, post-endotoxaemically, inhibition has detrimental effects on these parameters and is associated with a dramatic reduction in survival. Due to these late sequellae, it therefore has to be questioned whether selective NOS2 inhibition should be attempted during the early phases of endotoxaemic shock. 


\section{REFERENCES}

1. Bersten $A D$, Hersch $M$, Cheung $H$, Rutledge FS, Sibbald W]. The effect of various sympathomimetics on the regional circulations in hyperdynamic sepsis. Surgery 1992;112(3): 549-61.

2. Thiemermann C Nifric oxide and septic shock. Gen Pharmacol 1997;29(2):159-66.

3. Hibbs JBJ, Westenfelder $C_{s}$ Taintor R, Vavrin Z, Kablitz C, Baranowski RL, Ward JH, Menlove RL, McMurry MP, Kushner JP, et al. Evidence for cytokine-inducible nitric oxide synthesis from L-arginine in patients receiving interleukim-2 therapy. J Clin Inwest 1999;89(3):867-77.

4. Nathan C, Xie QW. Nitric oxide synthases: roles, tolls, and controls. Cell 1994;78(6):915-8.

5. Kirkeboen KA, strand $\emptyset \mathrm{A}$. The role of nitric oxide in sepsis-an overview. Acta Anaesthesiol.Scand 1999;43(3);275-88.

6. Grover R, Lopez A, Lorente IA, et al. Multi-center, randomized, placebo-controlled, double blind study of the nitric oxide synthase inhibitor 546C88: effect on survival in patients with septic shock. [Abstract] Crit Care Med 1998;27:(1)A33

7. Johnson ML, Billiar TR. Roles of nitric oxide in surgical infection and sepsis. World I Surg 1998;22(2):187-96.

8. Scott JA, Metha $S$, Duggan $M$, Bihari $A$, McCormack DG. Functional inhibition of constitutive nitric oxide synthase in a rat model of sepsis. Am J Respir.Crit Care Med 2002;165(10):1426-32.

9. Kilbourn $R G$, Szabo $C_{\text {; }}$ Traber DL. Beneficial versus detrimental effects of nitric oxide synthase inhibitors in circulatory shock: lessons learned from experimental and clinical studies. Shock $1997 ; 7(4) ; 235-46$.

10. Saetre T, Smiseth $O A$, Scholz $T$, Carlsen $H$, Nordsletten L, Aasen AO. Nitric oxide synthase inhibition reduces venous return in porcine endatoxemia. Am J Physiol 1996;271(40): $\mathrm{H} 1325-\mathrm{H} 1332$

11. Gundersen $Y$, Corso $C$, Leiderer R., Lilleaasen $\mathbb{P}$, Aasen $A O_{n}$ Messmer $K$. Use of selective and nonselective nitric oxide symthase inhibitors in rat endotoxemia: effects on hepatic morphollogy and function. Shock 1997;8(5):368-72.

12. Wang $Y$, Lawson $J A$, Jaeschke $H$. Differential effect of 2-aminoethyl-isotiourea, an inhibitor of the inducible nitric oxide synthase, on microvascular blood flow and organ injury in models of hepatic ischemia-reperfusion and endotoxemia. Shock 1998;10(1):20-5.

13. Bruins Ml, Lamers $W_{1}$, Meijer Al, Soeters PB, Deutz NE. In vivo measurement of nitric oxide production in porcine gut, liver and muscle during hyperdynamic endotoxaemia. $\mathrm{Br}$ J Pharmacol 2002;137(8):1225-36.

14. Ten Have GAM, Bost MCF, Suyk-Wierts JCAW, van den Bogaard AEIM, Deutz NEP. Simultaneous measurement of metabolic flux in portally-drained viscera, liver, spleen, kidney and hindquarter in the conscious pig. Lab Animals 1996:30:347-58.

15. Castillo $L$, Beaumier $L$, Ajami $A$, Young VR. Whole body nitric oxide synthesis in healthy mean determined from [15N]arginine-to-[15N]citrulline labeling. Proc Natl Acad Sci USA 1996;93:11460-5

16. Bruins MJ, Soeters $\mathrm{PB}$, Lamers WH, Deutz. NE. L-arginine supplementation in pigs decreases liver protein turnover and increases hindquarter protein turnover both during and after endotoxemia. Am J Clin Nutr 2002; 75(6):1031-44.

17. Wolfe, RR. Radioactive and stable isotope tracers in biomedicine. Principle and practice of kinetic analysils. 1. New York: Willey-Liss 1992; p.49 
18. van Eijk HM, Rooyakkers DR, Deutz NEP. Rapid rotitine determination of amino acids in pllasma by high-perfomance liquid chromatography with a 2-3 microns Spherisorb ODS 1 column. Chromatogr 1993;62011/143-8.

19. Bruins M, Soeters PB, Lamers WH, Meijer $A$, Deutz NE. L-arginine stupplententation n hypendynamic endotoxemic pigs: effect on nitric oxide synthesis by the different organs crit Care Med 2002;30(3):508-17.

20. Cheng $X$, Leung SW, Lo LS, Pang CC. Selective versus non-selective suppression of nitric oxide synthase on regional hemodynamics in rats with or without LPS-induced endotoxemia. Naunyn Schmiedebergs Arch Pharmacol, 2003;367:372-9.

21. Corso $\mathrm{CO}$, Gundersen $Y$, Dorger $M$, Lilleaasen $P$, Aasen $\mathrm{AO}$, Messmer $\mathrm{K}$. Effects of the nitric oxide synthase inhibitors $N(G)$-nitro-L-arginine methyl ester and aninoethylisothourea on the liver microcirculation in rat endotoxemia. Hepatol 1998;28:61-9.

22.. Pulido E), Shames BD, Fullerton DA, Sheridan BC, Selzman $\mathrm{CH}_{*}$ Gamboni-Robertson $\mathrm{F}$, Bensard DO, Mcintyre RC In. Differential inducible nitric oxide synthase expression in systemic and pulmonary vessels after endotoxin. Am I Physiol 2000;278:R1232-9.

23. Saetre $T$, Hovig $T$, Roger $M$, Gundersen $Y$, Aasen AO. Hepatocellular damage in porcine endotoxemia: beneficial effects of selective versus non-selective nitric oxide synthase inhibitiont Scand I Clin Lab invest 2001;6:503-12.

24. Southan GJ, Szabo C. Thiemermann C. Isothioureas: potent inhibitors of nitric oxide synthases with variable isoform selectivity, Br I Pharmacol 1995; 114(2):510-6.

25. Saetre T, Gundersen $Y$, Thiemermann $C_{b}$ Lilleaasen $P$, Aasen AO. Amino-isothiourea, a selective inhibitor of inducible nitric oxide synthase activity, improves liver circulation and oxygen metabolism in a porcine model of endotoxemia. Shock 1998;9(2):109-15.

26. Hrabak A, Bajort $T$, Southan Gl, Salzman AL, Szabo C. Comparison of the inhibitory effect of isothiourea and mercapto-alkylguanidine derivatives on the alternative pathways of arginine metabolism in macrophages. Life Sc: 1997;60(26):PL395-401.

27. Garvey EP, Oplinger JA, Tanoury G), Sherman PA, Fowler M, Marshall S, Harmon MF, Paith JE, Furfine ES. Potent and selective inhibition of human nitric oxide synthases, Inhibition by non-amino acid isothioureas. J Biol Chem 1994;269(43):26669-76.

28. Southan G), Szabo C. Selective pharmacollogical inhibition of distinct nitric oxide synthase isoforms. Biachem Pharmacol 1996;51(4):383-94.

29. Pittner $A$, Nalos $M$, Asfar $P$, et al. Mechanisms of inducible nitric oxide synthase (iNOS) inthibition-related improvement of gut mucosal acidosis during hyperdynamic porcine endotoxemia. Intensive Care Med 2003;29:(2)312-316.

30. Anonymous. Third European Consensus Conference in Intensive Care Medicine. Tissue hypoxia. How to detect, how to correct, how to prevent? Am J Respir.Crit Carre Med 1996;154(5):1573-8.

31. Ince $C$, Sinaasappel M. Microcirculatory oxygenation and shunting in sepsis and shock. Crit. Care Med 1999;27(7): 1369-77.

32. Saetre T, Hoiby EA, Aspelin T, Lermark G, Egeland T, Lyberg T. Aminoethyl-isothiourea, a nitric oxide synthase inhibitor and oxygen radical scavenger, improves survival and counteracts hemodynamic deterioration in a porcine model of streptococcal shock. Crit Care Med 2000;28:2697-706.

33. Gundersen $Y$, Corso $C O$, Leiderer $\mathbb{R}$, Dorger $M$, Lilleaasen $P$, Aasen $A O$, Messmer $K$. The nitric oxide donor sodium nitroprusside protects against hepatic microcirculatory dysfunction in early endotoxaemia. Intensive Care Med 1998;24:1257-63. 
34. Banning AP, Groves PH, Buttery LD, Wharton I, Rutherford RA, Black P, Winkler F, Polak JM, Lewis Ml, Drexler $H$. Reciprocal changes in endothelial and inducible nitric oxide synthase expression following carotid angioplasty in the pig. Atherosclerosis 1999;145:17-32.

35. Battista $S$, Bar $F$, Mengozzi $G_{n}$ Zanon $E$, Molino $G$. Hyperdynamic circulation in patients with cirrhosis: direct measurement of nitric oxide levels in hepatic and portal veins. I Hepatol $1997 ; 26: 75-80$. 


\section{CHAPTER 13}

Beneficial effects of L-arginine on

nitric oxide metabolism and hepatosplanchnic

perfusion during porcine endotoxaemia 


\section{ABSTRACT}

Background: The effects of L-arginine treatment on whole-body and hepatosplanchnic nitric oxide (NO) production and perfusion were studied prior to, during and after endotoxaemia in the pig.

Methods. In a randomised controlled trial, chronically instrumented female pigs $(20-25 \mathrm{~kg})$ received $3 \mathrm{\mu g} / \mathrm{kg} / \mathrm{h}$ lipopolysaccharide (LPS) intravenously and saline resuscitation, L-arginine $(3 \mathrm{mg} / \mathrm{kg} / \mathrm{h})$ or saline was administered intravenously starting $12 \mathrm{hrs}$ prior to a $24 \mathrm{hrs}$ LPS infusion and continued for $24 \mathrm{hrs}$ after stopping the endotoxiln infusion. Whole-body appearance rates, and portal-drained viscera (PDV) and liver fluxes of arginine, citrulline, NO and whole-body arginine de novo synthesis were determined by primed, stable isotope infusion of ${ }^{15} \mathrm{~N}_{2}$-arginine and ${ }^{13} \mathrm{C}-\mathrm{H}_{2}$-citrulline, Hepatosplanchnic blood flow and oxygen consumption $\left(\mathrm{NO}_{2}\right)$ was determined using a primed-continuous infusion of para-aminohippuric acid and intestimal intramucosal $\mathrm{pCO}_{2}$, respectively.

Results. Arginine supplementation per se did not affect blood flow in the aorta and hepatic artery, but increased that in the portal vein. Furthermore, it increased NO production at the whole-body level and PDV, but not in the liver. Arginine supplementation during subsequent exposure to LPS decireased peripheral resistance, increased cardiac output, reduced the intestinal intramucosal $\mathrm{pCO}_{2}$, and prevented an increase in PAP and glycolytic energy production. These beneficiall effects of arginine supplementation were accompanied by an increased NO synthesis at the whole-body level and in the liver, together with a temporarily decreased NO synthesis in the PDV.

Conclusions: Arginine treatment starting prior to endotoxaemia appears to be beneficial, because it enhances NO synthesis during endotoxaemia and improves hepatosplanchnic perfusion and oxygenation without deleterious systemic side effects. 


\section{INTRODUCTION}

High-risk surgery and sepsis are often accompanied by an increased need for, and a decreased supply of arginine, so that plasma arginine levels often become decreased under these conditions $(1,2)$. When plasma arginine levels decline, L-arginine may become rate-limiting as substrate for nitric oxide (NO) synthesis, since arginine transporters with an affinity for arginine in the same range as its circulating concentration determine intracellular arginine availability (3).

In addition, the expression of the constitutive NOS1 and NOS3 isoforms is suppressed during sepsis, although NOS2-dependent NO production can potentially compensate for this deficiency (4). Because a basal NO production is necessary for normal neurotransmission, organ perfusion, and immune defense, arginine supplementation has been suggested as a therapeutic option in these circumstances. On the other hand, if NOS2 is strongly induced as in sepsis, arginine supplementation may result in the production of large quantities of NO, which in turn can cause vascular hyporesponsiveness and hypotension, remote tissue injury and myocardial failure, symptoms that are frequently observed in septic shock (5). Selective inhibition of NOS2 does, indeed, initially reduce vascular hyporesponsiveness during sepsis, but as we recently demonstrated (6), such inhibition subsequently has detrimental effects on hepatosplanchnic metabolism and survival.

Whether or not arginine supplementation is beneficial for conditions resembling sepsis is usually evaluated by beginning the administration of arginine some time after the infusion of endotoxin has started, that is, when NOS2 expression is already elevated and arginine availability may already be limiting (7). In a protocol otherwise resembling the present study, we observed that supplementation of arginine beginning $8 \mathrm{hrs}$ after the start of endotoxin infusion increased the liver and visceral NO production. In the present study, we wished to evaluate the effects of L-arginine supplementation prior to LPS treatment on NO production and NO-dependent functions during subsequent endotoxaemia. This protocol would simulate the clinical situation of providing arginine prior to high-risk surgery. We find that arginine pre-treatment increases whole-body, visceral and liver NO production both prior to and during LPS treatment. Furthermore, intestinal and liver perfusion, and intestinal intramucosal $\mathrm{pCO}_{2}$ improved during $\mathrm{L}$-arginine treatment.

\section{MATERIALS AND METHODS}

\section{Animals}

Female crossbred pigs (Yorkshire $\times$ Dutch Landrace: weighing 20-25 kg) were individually housed, had free access to water and received $1 \mathrm{~kg}$ of regular pig feed ( $16 \%$ crude protein; Landbouwbelang, Roermond, The Netherlands) daily in 
the morning, supporting a growth rate of $300 \mathrm{~g}$ body weight per day. The Anumal Ethics Committee of the Maastricht University approved the study.

\section{Surgical Procedure}

Animals were fasted overnight. After the pigs were premedicated with $10 \mathrm{mg}$ $\mathrm{kg}$ azoperone (Stresnil, Janssen Pharmaceutica, Besse, Belgium) intramuscullarly, anaesthesia was induced with a mixture of $\mathrm{N}_{2} \mathrm{O} / \mathrm{O}_{2}(1: 2$, by vol) and halothane $(0.8 \%)$. After intubation, the pigs were intravenously administered $6.25 \mathrm{mg} / \mathrm{kg}$ Lincomycin (A.U.V., Cuyk, The Netherlands) as bactericidal prophylaxis and 12.5 mig/kg Spectinomycin (A.U.V.) as bacteriostatic prophylaxis. Flunixine $50 \mathrm{mg} / \mathrm{kg}$ Finadyne Schering-Ploegh, Brussels, Belgium) was administered intravenously for postoperative analgesia. During surgery, anaesthesila was maintained with a mixture of $\mathrm{N}_{2} \mathrm{O} / \mathrm{O}_{2}$ and halothane and fluid supplementation was provided with intravenous Ringer's lactate (Lactetrol, Aesculaap, Boxtel, The Netherlands).

After a midline laparotomy, 7 catheters $(80 \mathrm{~cm}$-long, ID $1.0 \mathrm{~mm}$, OD $1.8 \mathrm{~mm}$; Tygon, Westvaco, Cleveland, OH, USA) were inserted in the blood vessels as reported previously ( 8 ). In brief, catheters for blood sampling and blood pressure monitoring were placed in the abdominal aorta (above the right renal vein), which, in combination with the catheters in the portal and hepatic veins, served for metabolic measurements across the portal-drained viscera and the splanchnic organs, respectivelly. Catheters inserted in the splenic vein and the abdominal aorta (above the bifurcation) served to infuse para-aminohippuric acid (PAH) for plasma flow determination. For infusion of isotopes and endotoxin, a catheter was implanted in the caval vein above the right renal vein. $A$ thermodilution catheter (7F, Baxter Edwards Critical Care, Irvine, CA, USA) was inserted via the femoral vein into the pulmonary artery to monitor cardiac output and pulmonary pressures. In addition, a gastrostomy catheter (I.D. $1.6 \mathrm{~mm}$, O.D. $4.8 \mathrm{~mm}$, Tygon) was placed. All catheters were tunneled through the abdominal wall and skin and sutured to the skin using Vicryl 3-0. The catheters were filled with $20 \mathrm{mg} / \mathrm{ml}$ gentamicin (A.U.V., Cuyk, the Netherlands) and $225 \mathrm{U} / \mathrm{ml}$ chymotrypsin (Merck, Darmstadt, Germany) in $0.9 \%$ saline, to avoid catheter tip infections and to maintain patency. Finally, a jejunostomy was made by transecting the jejunum $20-25 \mathrm{~cm}$ distal to the ligament of Treitz and making an end-to-side anastomosis $10 \mathrm{~cm}$ distal to the transected distal end using Prolene 4.0 suture. The distal end was then tunnelled through the abdominal wall and skin and sutured to the skin using Prolene 3.0.

\section{Postoperative care}

Postoperative care was standardised as previously described (8). A canvas harness was fitted to each pig to protect the catheters and to allow easy handling of the animal. From the third day onward, the pigs were fed $1 \mathrm{~kg}$ of feed daily. During the whole recovery period (14 days), the animals remained healthy without signs of 
infection. In a pilot study 4 weeks after the operation, the position of the catheter tips was checked under anaesthesia using fluoroscopy.

\section{Experimental protocol}

The experiment started 14 days after surgery (Figure 1). Twelve hours before starting the infusion of endotoxin, the pigs received in a randomised, but non-blinded fashion either an intravenous infusion of $3 \mathrm{mg} / \mathrm{kg} / \mathrm{h}$ of L-arginine (iso-osmolar, $5.3 \mu \mathrm{mol} / \mathrm{kg} / \mathrm{h}, \mathrm{pH} 7.3 ; \mathrm{n}=8$ ), or $0.9 \%$ saline $(\mathrm{n}=7$ ) at an equal infusion rate. Arginine and saline infusions were continued throughout the whole experiment. After fasting overnight, the pigs were placed in a movable cage (before the start of the arginine infusion). After taking initial blood samples, all pigs received $3 \mu \mathrm{g} / \mathrm{kg} / \mathrm{h}$ lipopolysaccharide endotoxin (LPS) from Escherichia coli (055:B5, Sigma Chemicals Co., St. Louis, MO, USA) dissolved in saline for $24 \mathrm{~h}$ via the inferior caval vein catheter. The endotoxin dose used makes this model a zero mortality model (1). All pigs received $0.9 \%$ saline as a fluid support: $30 \mathrm{ml} / \mathrm{kg} / \mathrm{h}$ during the first $8 \mathrm{hrs}$ and $20 \mathrm{ml} / \mathrm{kg} / \mathrm{h}$ during the next $16 \mathrm{hrs}$ of endotoxin infusion. During the experimental period, the pigs were kept fasted. At the end of each experiment, the pig was sacrificed using a lethal dose of thiopental (Nesdonal, Rhône-Poulenc Pharma, Amstelveen, The Netherlands).

\begin{tabular}{llllll} 
& + & 1 & & \\
\hline Time (h) 0 & 12 & 20 & 36 & 60
\end{tabular}

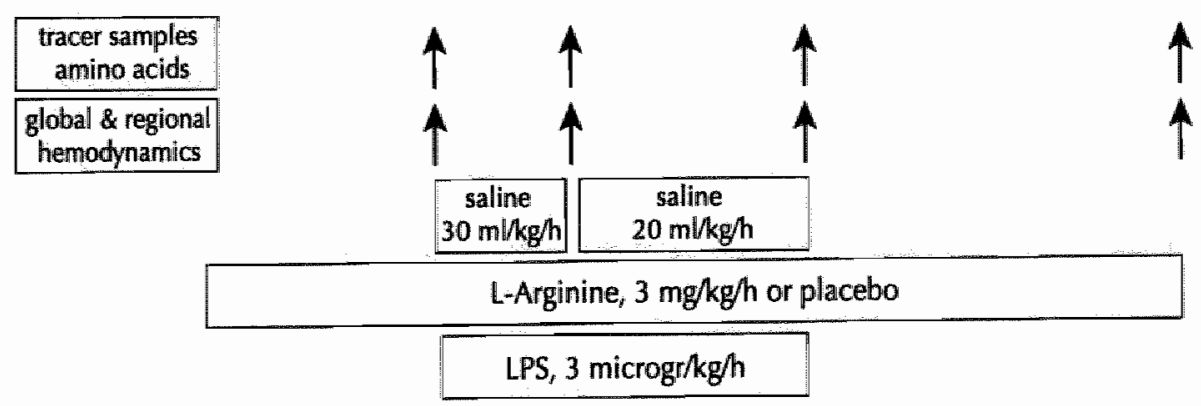

LPS, lipopolysaccharide (endotoxin)

Figure 1 Illustration of experimental design 


\section{Infusion and sampling protocol}

\section{Stable isotopes}

Before the start of the isotope infusions, background blood samples were taken. Two hours after the start of the primed-constant infusion of the isotopes through the inferior caval vein catheter, steady state conditions were obtained $(9,10)$. Therefore, blood was sampled $2 \mathrm{~h}$ after the start of the primed-constant infusion of the isotopes. Prime and tracer infusion concentrations were based on the whole-body tracee appearance (11). L-[guanidino- ${ }^{15} \mathrm{~N}_{2}$ ] arginine ( $\mathrm{N}_{2}$-arginine: infusion: $6.4 \mu \mathrm{mol} / \mathrm{kg} / \mathrm{h}$, prime: $3.2 \mu \mathrm{mol} / \mathrm{kg}$ ), and $\mathrm{L}$-[ureido${ }^{13} \mathrm{C}, 5,5-{ }^{2} \mathrm{H}_{2}$ ) citrulline $\left(\mathrm{C}_{1}-\mathrm{D}_{2}\right.$-citrulline: infusion: $0.3 \mu \mathrm{mol} / \mathrm{kg} / \mathrm{h}$, prime: 1.5 pmol $/ \mathrm{kg}$ ) were purchased from Mass Trace (Woburn, USA). Isotopes were prime-infused; 2 hrs prior to blood sampling $0,8,24$, and 48 hrs after start of endotoxin infusion. Blood was sampled from catheters in the abdominal aorta, the portal vein, and the hepatic vein before isotope infusions ceased.

\section{PAH}

One hour after the start of the primed-constant infusion $25 \mathrm{mM}$ PAH $(5 \mathrm{ml}$ bolus followed by infusion at a rate of $40 \mathrm{ml} / \mathrm{h}$ through the splenic vein and the aorta catheters; A 1422, Sigma Chemicals Co), a steady-state condition for PAH was obtained (8). Therefore, blood was sampled at least $2 \mathrm{~h}$ after the start of the primed-constant infusion of PAH.

\section{Tonometry}

A tonometry catheter (14 F, Datex Ohmeda, Finland) was inserted through the jejunostomy for at least $25 \mathrm{~cm}$. A volume of $4.5 \mathrm{ml}$ of $0.9 \%$ salline was instilled into the balloon of the catheter and equilibrated for at least $30 \mathrm{~min}$. After equilibration and just prior to the endotoxin infusion, the saline was withdrawn from the balloon and the first $1.5 \mathrm{ml}$ was discarded. The $\mathrm{pCO}_{2}$ of the saline was measured as for other blood gasses (see sample processing). Tonometric measurements were repeated at $0,8,24$, and $48 \mathrm{~h}$ after the start of the endotoxin infusion.

\section{Cardiac output}

Prior to, and at $0,8,24$, and 48 h after start of the endotoxin infusion, 3 boluses of $5 \mathrm{ml}$ ice-cold saline each were administered to estimate the cardiac output using the thermodillution technique with a cardiac output computer (9520A, Baxter Edwards Critical Care, Irvine, CA, USA). At these time-points, mean systemic pressure (MAP) and mean pulmonary arterial pressure (PAP) were measured in triplicate, after 'zeroing' all pressure transducers to the level of the heart.

\section{Sample processing}

Promptly after sampling, blood was distributed in pre-chilled, heparinised tubes (Sarstedt, Nümbrecht, Germany) on ice. Haematocrit was determined to enable 
calculation of plasma flow from blood flow. For blood gas analysis (arterial $\mathrm{pH}$, $\mathrm{PO}_{2}$ and $\mathrm{PCO}_{2}, \mathrm{HCO}_{3}$, and base excess), $0.2 \mathrm{ml}$ blood was sealed airtight in heparinized $1 \mathrm{ml}$ syringes and immediately analysed on an automatic blood gas system (Acid Base Laboratory (ABL3), Radiometer, Copenhagen, Denmark) and corrected for pig haemoglobin and core temperature of the animal. The temperature of the pig was measured in the central venous blood using the pulmonary artery flotation catheter.

For PAH determination, $300 \mu$ l of whole blood was added to $600 \mu \mathrm{l}$ of $12 \%$ (w/ v) trichloroacetic acid, mixed thoroughly, and centrifuged $\left(4{ }^{\circ} \mathrm{C}\right.$ for 5 minutes at $8,500 \mathrm{~g}$ ). For glucose and lactate concentration determinations, $900 \mu \mathrm{l}$ plasma was added to $90 \mu \mathrm{l} 50 \%$ trichloroacetic acid solution. For analysis of amino-acid concentrations, plasma was deproteinised by mixing $500 \mu \mathrm{l}$ with $20 \mathrm{mg}$ dry sulfosalicylic acid. All samples were frozen in liquid nitrogen and stored at $-80^{\circ} \mathrm{C}$ until further analysis.

\section{Biochemical analysis}

PAH was detected spectrophotometrically after deacetylation of the supernatant at $100{ }^{\circ} \mathrm{C}$ for 45 minutes (8). Plasma glucose, pyruvate and lactate concentrations were determined by automated HPLC (Pharmacia, Woerden, The Netherlands). Concentrations and enrichments of amino acids were calculated as tracer-to-tracee ratios (TTR) and were determined by a fully automated liquid chromatography-mass spectrometry system (LC-MS, Thermoquest LCQ, Veenendaal, The Netherlands) after pre-column derivatization with o-phthaldialdehyde (12).

\section{Calculations}

The whole-body rate of appearance $\left(\mu \mathrm{mol} / \mathrm{kg}\right.$ bw/min) of L-arginine $\left(\mathrm{Q} \mathrm{Ra}_{\mathrm{ARG}}\right)$ and citrulline $\left(Q \mathrm{Ra}_{\mathrm{CIT}}\right.$ ) were derived from formula (1) using the $\mathrm{N}_{2}$-arginine and the $C_{1} D_{2}$-citrulline isotope, respectively. The tracer infusion rate is represented by $I$ and divided by $\pi R_{A}$ which is the tracer-to-tracee ratio in the arterial plasma (11). TTR values were corrected for background values.

(1) $\mathrm{QRa}=1 / \pi T R_{\mathrm{A}}$

The endogenous rate of $\mathrm{Q} \mathrm{Ra}_{\mathrm{ARG}}$, assumed to reflect de novo $\mathrm{L}$-arginine synthesis plus $L$-arginine appearance from protein breakdown, was estimated by correcting the total $Q R_{A R G}$ for the contribution of L-arginine infused. Formula (2) was used to calculate the rate of whole-body synthesis of $N O\left(Q R a_{N O}\right)$ based upon the plasma isotope transfer from $\mathrm{N}_{2}$-arginine to $\mathrm{N}_{1}$-citrulline.

(2) $\mathrm{QRa} \mathrm{N}_{\mathrm{NO}}=\mathrm{QR \textrm {R } _ { \mathrm { CIT } }} \times\left(T T \mathrm{R}_{\mathrm{AN1}-\mathrm{CIT}} / \mathrm{TTR}_{\mathrm{AN} \text { N2-ARC }}\right)$ 
$T T R_{A N 1-C I T}$ and $T T R_{A N 2-A R G}$ represent the $T T R$ of $N_{1}$-citrulline and $\mathrm{N}_{2}$-arginine in arterial plasma, respectively.

The portall-drained viscera (PDV) are defined as the total of all portal-drained organs that; apart from the spleen, stomach and pancreas, largely represent the intestines. The splanchnic area is the sum of the portal-drained viscera and liver, so that liver values could be obtained by subtracting portal-drained viscera from splanchnic values. L-arginine and NO metabolism across the portal-drained viscera, the splanchnic region and the liver were calculated in a two-compartment model, as was described previously (1).

Plasma flow across liver and portal-drained viscera was calculated using formula (1) based on the principle of indicator dilution methods in which I represents the rate of the $\mathrm{PAH}$ infusion near the organ. $[\mathrm{PAH}]_{V}$ and $[\mathrm{PAH}]_{A}$ represent the concentration $(\mu \mathrm{mol} / \mathrm{l})$ of $\mathrm{PAH}$ in the venous and arterial blood, respectively. The venous [PAH] dilution depends on the organ blood flow rate. By sampling upstream and downstream of the organ, at the arterial and venous side, respectively, the organ blood flow can be calculated in (3). The blood flow was converted to plasma flow by using equation (4).

(3) Flow $_{\text {BLOOD }}=1 /\left([\mathrm{PAH}]_{V}-[\mathrm{PAH}]_{\mathrm{A}}\right)$

(4) Flow $_{\text {PLASMA }}=$ flow $_{\text {BLOOD }} \times 100 /(100-$ hematocrit $)$

Amino acid net balance (NB) was calculated according to formula (5) by multiplying the mean plasma flow with the difference between $[\mathrm{V}]$ and $[\mathrm{A}]$ that represent the venous and arterial plasma concentration of the amino acid, respectively. Therefore, a negative NB represents net influx of amino acid into, and a positive NB net efflux of amino acid from the organ.

(5) $N B=$ flow $x([\mathrm{~V}]-[A])$

In fed pigs, L-arginine NB across the portal-drained viscera was corrected for the arginine in the enteral nutrition that was incorporated into protein or degraded in the portal-drained viscera.

The disposal and production rate of arginine and citrulline of the PDV and liver were calculated using equations (6) to (8).

(6) Disposal ${ }_{\mathrm{ARG}}=\mathrm{nb}_{\mathrm{N2}-\mathrm{ARG}} / \mathrm{TTR}_{\mathrm{VN2} \text {-ARC }}$

(7) $\mathrm{nb}_{\mathrm{N} 2-\mathrm{ARG}}=\left(\mathrm{PDV}\right.$ flow $\left.\times[\mathrm{V}] \times \mathrm{TTR}_{\mathrm{V}}\right) \cdot\left(\mathrm{PDV}\right.$ flow $\left.\times[\mathrm{A}] \times \mathrm{TTR}_{\mathrm{A}}\right)$

(8) Production $_{A R G}=N B+$ Disposal $_{A R G}$

The rate of $\mathrm{NO}$ synthesis by individual organs ( $\mathrm{Syn}_{\mathrm{NO}}$ ) can be calculated from conversion of $\mathrm{N}_{2}$-arginine to $\mathrm{N}_{1}$-citrulline across that organ. The term $\mathrm{nb} \mathrm{N}_{\mathrm{N}-\mathrm{crr}}$ represents the tracer net balance of the $N_{1}$-citrulline isotope in arterial and venous plasma, respectively. Because of possible fractional extraction of citrulline and, therefore, $N_{1}$-citrulline $\left(\mathrm{FE}_{\mathrm{CIT}}\right.$ by the organ, $\mathrm{nb}_{\mathrm{N} 1-\mathrm{CIT}}$ must be corrected for the loss of $N_{1}$-citrulline tracer across the organ, which is possible by using 
the $C_{1} D_{2}$-citrulline isotope in equation (9). The term (1 - FE) represents the fraction of total amino acid that bypasses metabolism in the organ and so appears in the output and is used for correction of the $N_{1}$-citrulline $n b(10)$. Organ Syn $N_{N}$ is derived from formula (11), in which $T T R_{A N 2-A R C}$ represents the arterial $N_{2}$-arginine enrichment. The TTR of $\mathrm{N}_{2}$-arginine in the arterial plasma free pool was assumed to be the same as the TTR of $\mathrm{N}_{2}$-arginine in the direct precursor pool of $\mathrm{NO}$ synthesis.

(9) $\left.\mathrm{FE}_{\mathrm{CIT}}=\left(T T R_{\mathrm{A}} \times[\mathrm{A}]-T \mathrm{TT} \mathrm{R}_{\mathrm{V}} \times[\mathrm{V}]\right) / T T R_{\mathrm{A}} \times[\mathrm{A}]\right) \mathrm{C1D} 2-\mathrm{CIT}$

(10) $\mathrm{nb}_{\mathrm{N1}-\mathrm{CIT}}=$ flow $\times\left\{[\mathrm{V}] \times T T R_{\mathrm{V}}-[\mathrm{A}] \times T T R_{\mathrm{A}} \times\left(1-\mathrm{FE}_{\mathrm{CIT}}\right)\right\}_{\mathrm{N1}-\mathrm{CIT}}$

(11) $\mathrm{SYn}_{\mathrm{NO}}=\mathrm{nb}_{\mathrm{N1}-\mathrm{CIT}} / \mathrm{TTR}_{\mathrm{AN} 2-\mathrm{ARG}}$

\section{Statistics}

Results are presented as mean \pm SEM. Levels of significance were set at $p<0.05$. The data were subjected to multiple-way ANOVA analysis to test the effect of time and to compare group differences during the 60hrs study period, using as within-factor time with 4 levels (repeated measures: $t=12,20,36$, and 60hrs) and as between-factors treatment (arginine treatment or control). Alpha error values are reported in Tables and Figures, in which the first $p$-value represents the significance at $t=12 \mathrm{hrs}$, the $p$-values on the second row represents the significance from $t=12$ until $36 \mathrm{hrs}$ and the third row the significance from $t=12 \mathrm{hrs}$ compared to $t=60 \mathrm{hrs}$, and where $P_{T}$ indicates the significance of repeated measures over time, $P_{T \times C}$ the interaction of repeated measures and treatment group (time $x$ group) and $P_{C}$ the effect of arginine treatment.

\section{RESULTS}

\section{Animals}

In control animals, body weight rose from $22.2 \pm 0.9$ to $26.4 \pm 1.4 \mathrm{~kg}$ during infusion of LPS and saline, and declined to $25.0 \pm 1.5 \mathrm{~kg}$ after cessation of the infusions $\left(P_{T}=0.01\right)$. These changes in body weight were not significantly different from those observed in arginine-treated animals: from $23.9 \pm 0.9$ to $28.0 \pm 1.9 \mathrm{~kg}$ during infusion of LPS and saline, and back to $25.2 \pm 1.7 \mathrm{~kg} 24 \mathrm{hrs}$ after stopping these infusions $\left(P_{T_{x G}}=0.8\right)$. Compared to baseline values, the central temperature was increased during LPS infusion (20-36hrs) in both groups, implying that arginine supplementation did not prevent the development of fever $\left(P_{T \times G}=0.9\right)$. 


\section{Global haemodynamic parameters}

\section{Mean arterial pressure (MAP)}

Infusion of arginine alone did not change MAP (Table 1, 12hrs). During LPS infusion, the MAP decreased in all animals, but particularly in the arginine-treated group (Table 1, 20 and 36hrs; $P_{\mathrm{T}}=0.05$ ). In the control group, MAP returned to pretreatment values within 24 hrs after discontinuing LPS infusion, but in the arginine-treated group MAP remained depressed (Table 1, 60hrs; $P_{T}=0.09$ ).

\section{Cardiac index (CI)}

$\mathrm{Cl}$ tended to be lower in arginine-treated animals (Table 1, $12 \mathrm{hrs} ; \mathrm{P}_{\mathrm{T}}=0.3$ ). The $\mathrm{Cl}$ increased significantly upon LPS infusion in both experimental groups (Table 1, 20 and 36 hrs; $P_{G}=0.001$ ), but the increase peaked already at 8 hrs after beginning the LPS infusion in arginine-treated animals. After cessation of the LPS treatment, $\mathrm{Cl}$ returned to pretreatment levels in control animals, but remained elevated in arginine-treated pigs (Table 1, 60hrs).

\section{Pulmonary arterial pressure (PAP)}

PAP was not affected by arginine supplementation (Table 1, 12hrs) and increased only in the control group during LPS infusion (Table 1, 20 and $36 \mathrm{hrs} ; P_{C}=0.008$ ).

\section{Lactate}

Arterial lactate concentration was not affected by arginine treatment per se (Table 1, $12 \mathrm{hrs})$, but significantly increased during LPS treatment in the control group only (Table 1, 20 and $36 \mathrm{hrs} ; P_{G}<0.0001$ ). In the latter group, lactate concentrations returned to pretreatment values within $24 \mathrm{hrs}$ after cessation of LPS infusion $\left(P_{\mathrm{TxC}}=0.02\right)$.

\section{Global metabolic parameters}

\section{Arginine}

Arginine supplementation increased whole-body arginine appearance by approx. 4 $\mu \mathrm{mol} / \mathrm{kg} / \mathrm{min}$ (Figure 2, panel $\mathrm{A}, 12 \mathrm{hrs} ; \mathrm{P}_{\mathrm{G}}<0.0001$ ), but increased whole-body de novo arginine biosynthesis only 1.6-fold (Figure 2, panel $D ; P_{G}=0.02$ ). Endotoxin infusion did not affect the rate of arginine appearance in either experimental group $\left(P_{T \times C}=0.8\right)\left(P_{G}<0.0001\right)$, but depressed the de novo arginine synthesis in the arginine-treated group to that found in the control group $\left(\mathrm{P}_{\mathrm{TxC}}=0.2\right)$.

Arterial levels of arginine were significantly higher at baseline (Table $1 ; P=0.04$ ) and remained significantly elevated during the experiment $P_{\mathrm{G}}=0.01$ and 0.04). Levels of arginine tended to decrease during the endotoxin infusion in arginine-supplemented animals. 


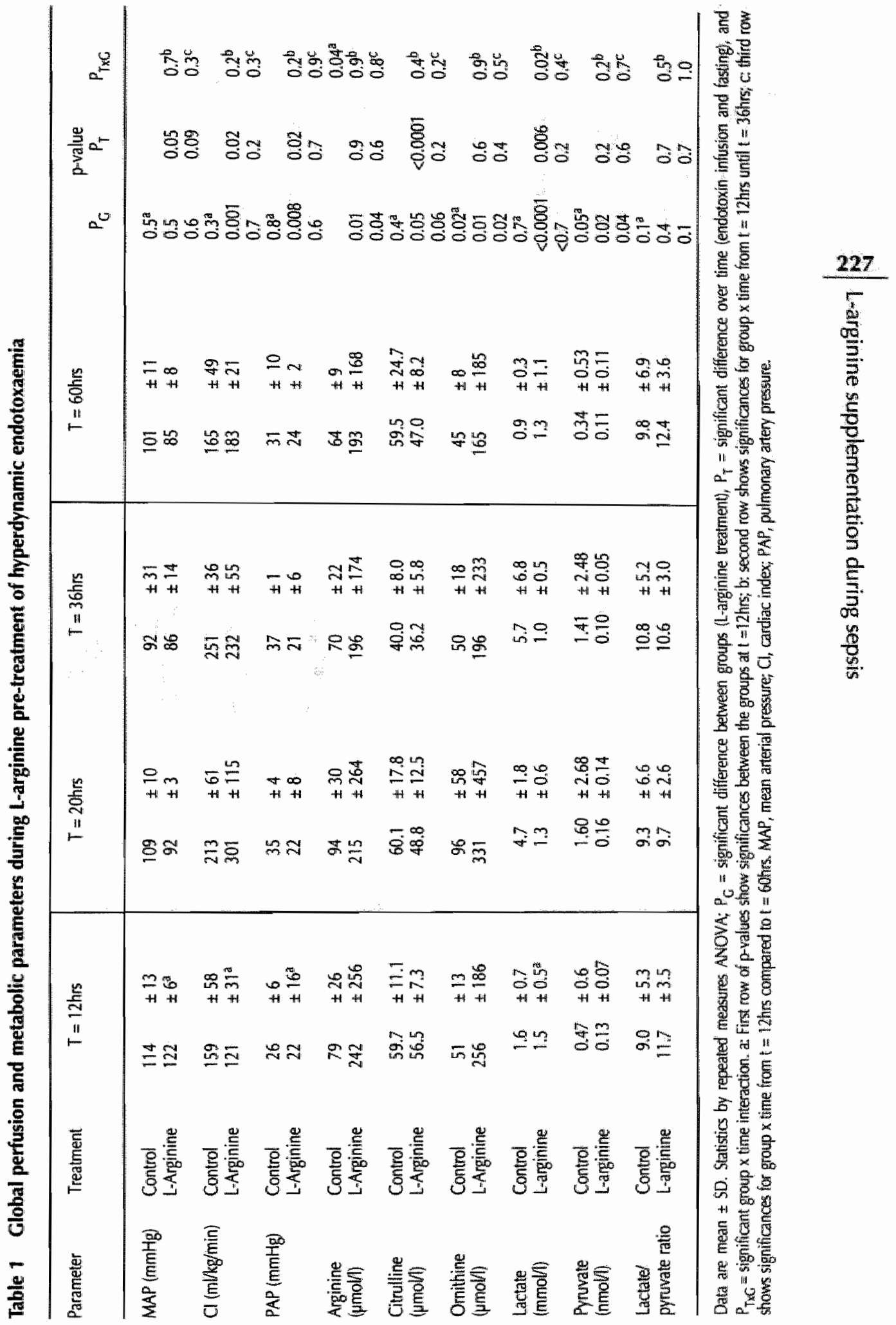



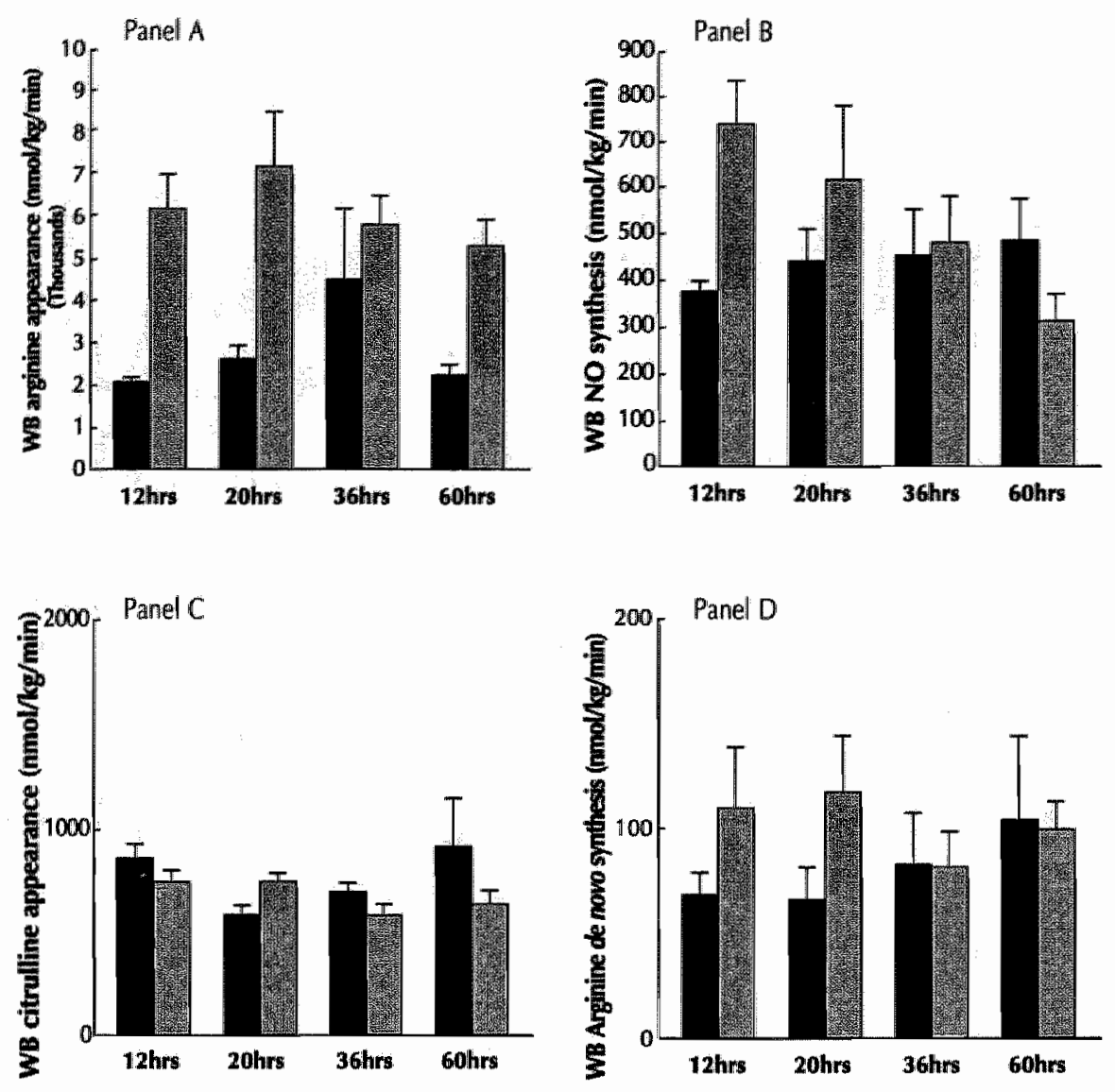

Data are presented as mean \pm standard error. Panel A: change in whole-body (WB) arginine appearance in control (solid bars) and $\mathrm{L}$-atginine-supplemented (shladed bars) animals; statistics by multiple-way $A N O N A_{\text {; }}$ pre-endotoxin $t=12 \mathrm{hrs}$, $P=0.001 ;$ during endotoxin infusion $P_{G}<0.0001, P_{T}=0.7, P_{T \times C}=0.8$; post-endatoxin infusion $P_{G}<0.0001, P_{T}=0.5$, $\mathrm{P}_{\mathrm{TKG}}=0.3$.

Panel $B$ : change in WB NO synthesis; pre-endotoxin $t=12 \mathrm{hrs,} P=0.02$; during endotoxin infusion $\mathrm{P}_{G}=0.03, \mathrm{P}_{\mathrm{T}}=0.5$, $P_{T \times G}=0.4$; post-endotoxin inlusion $P_{G}=0.2, P_{T}=0.006, P_{T \times C}=0.003$.

Panel $C$ : change in WB cituline appearance; pre-endotoxin $t=12 \mathrm{hrs}, P=0.5$; during endiotoxin infusion $P_{C}=0.6$, $P_{T}=0.003, P_{T X C}=0.05$; post-endotoxin infusion $P_{G}=0.06, P_{T}=0.4, P_{T \times G}=0.3$ *

Panel $D$ : change in WB arginine de novo synthesis; pre-endotoxin $t=12 \mathrm{hrs}, P=0.6$; during endotoxin infusion $P_{C}=0.02$, $P_{T}=0.8, P_{T \times C}=0.2 ;$ post-endotoxin infusion $P_{G}=0.3, P_{T}=0.6, P_{T \times G}=0.2$

Figure 2 Whole-body arginine-NO metabolism during L-arginine and saline (controls) pre-treated hyperdynamic endotoxaemia 
Arginine infusion doubled whole-body NO synthesis (Figure 2, panel $B_{*}, 12 \mathrm{hrs}$; $P=0.02$ ). LPS treatment did not significantly increase whole-body NO synthesis in the control group, but decreased it in the arginine-treated group to levels seen in the control group (Figure 2, panel B, 20 and 36hrs; $P_{G}=0.03$ ). During the first $24 \mathrm{hrs}$ after discontinuing LPS treatment, NO synthesis remained unchanged in the control group, but continued to decrease in the arginine-supplemented group to approx. $65 \%$ of that in the control group (Table 1, 60hrs; $\mathrm{P}_{\mathrm{TxG}}=0.003$ ).

\section{Citrulline}

Whole-body citrulline appearance was not affected by $12 \mathrm{hrs}$ of arginine supplementation (Figure 2, panel C, $12 \mathrm{hrs}$ ), but significantly decreased during LPS infusion in both experimental groups (Figure 2, panel C, 20 and 36hrs; $P_{\mathrm{T}}=0.003$ ). Whereas whole-body citrulline appearance returned to control values within $24 \mathrm{hrs}$ after discontinuing LPS treatment in the control group, it remained depressed in the arginine-treated group (Figure 2, panel $\mathrm{C}, 60 \mathrm{hrs} ; \mathrm{P}_{\mathrm{T} x \mathrm{G}}=0.3$ ). Arginine infusion tended to decrease arterial citrulline levels during the experiment (Table $\left.1 ; P_{C}=0.05\right)$.

\section{Ornithine}

Arginine infusion increased ornithine levels 5 -fold (Table 1; $P=0.02$ ). Endotoxin infusion did not infuence this increased level, but ornithine levels remained significantly higher $\left(\mathrm{P}_{\mathrm{G}}=0.01\right)$.

\section{Hepatosplanchnic perfusion parameters}

\section{Portal-drained viscera (PDV)}

The flow through the portal-drained viscera (PDV) increased during arginine supplementation (Figure 3 , panell $A, 12 \mathrm{hrs} ; \mathrm{P}=0.03$ ). LPS infusion did not affect PDV perfusion in control animals, but increased PDV perfusion in arginine-supplemented animals, although with a delay of at least Bhrs (Figure 3, panel $A, 20$ and 36hrs; $P_{G}<0.0001$ ). After stopping LPS, PDV flow returned to control levels (Figure 3, panel $A, 60 \mathrm{hrs} ; P_{T \times C}=0.8$ ).

\section{Liver}

In arginine-treated animals, liver flow was increased (Figure 3, panel B, 12 hrs; $P=0.4$ ). Liver perfusion increased significantly upon LPS infusion (Figure 3 , panel $B, 20$ and $36 \mathrm{hrs} ; P_{G}=0.01$ ), but remained higher in arginine-treated animals than in controls $\left(P_{G}=0.01\right)$. Flow through the hepatic artery did not change upon arginine supplementation alone (Figure 3 , panel $C, 12 \mathrm{hrs}$ ), but increased significantly upon LPS treatment (Figure 3, panel C, 20 and 36hrs; $P_{T}=0.004$ ), although this response took longer in control than in arginine-treated animals $\left(P_{T \times G}=0.02\right)$. Hepatic arterial flow remained elevated for at least $24 \mathrm{hrs}$ after stopping LPS treatment in both control and arginine-treated groups (Figure 3, panel C, 60hrs; $P_{T}=0.03$ ). 

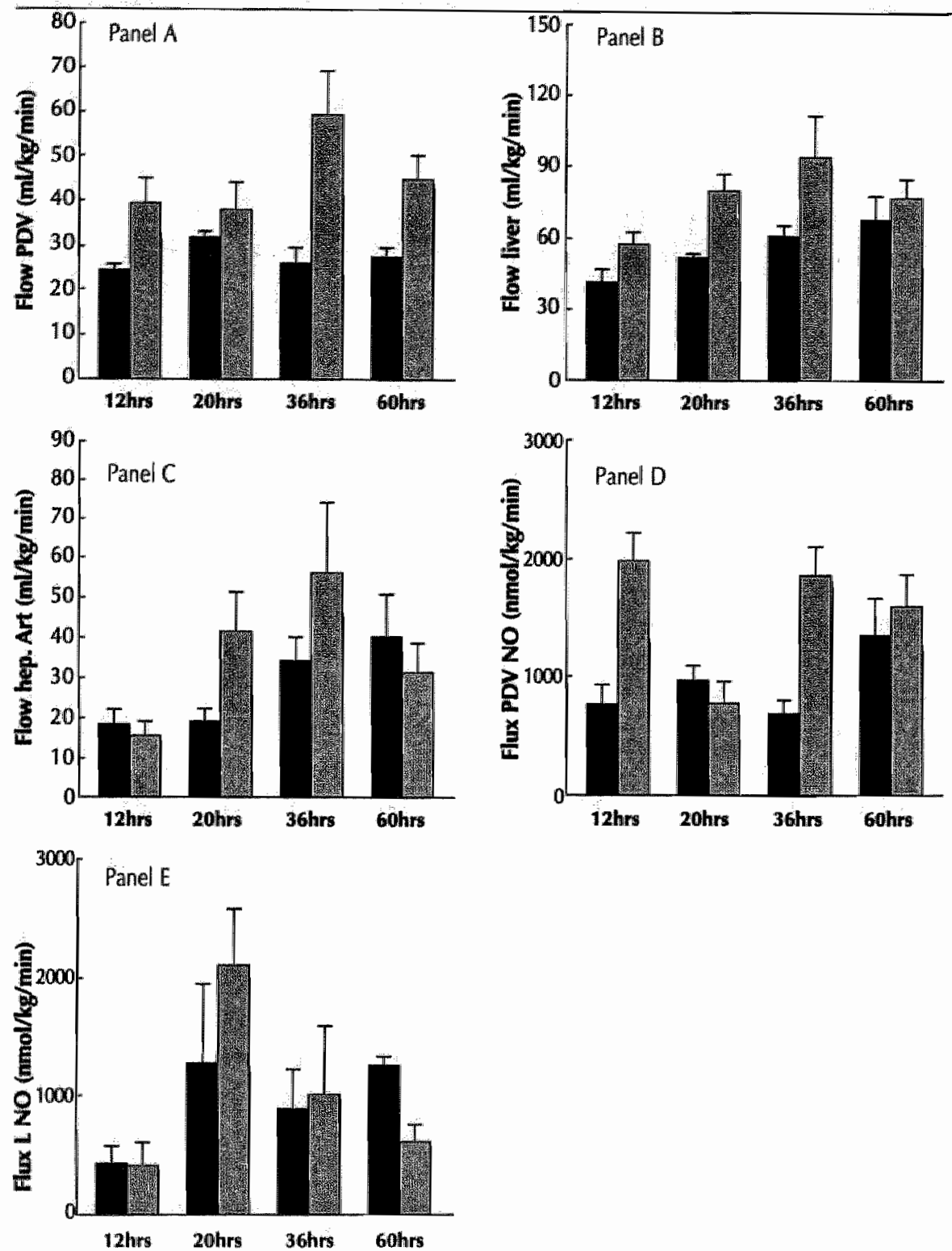

Data are presented as mean \pm standard error. Panel A: change in portally-drained viscera (PDV) flow in control (solid bars) and $\mathrm{L}$-arginine-supplemented (shaded bars) animals; statistics by mulltiple-way $A N O V A$; pre-endotoxin $t=12 \mathrm{hrs}, \mathrm{P}=0.03$; during endotoxin infusion $\mathrm{P}_{\mathrm{G}}<0.0001, \mathrm{P}_{\mathrm{T}}=0.02, \mathrm{P}_{\mathrm{TXG}}=0.008$; post-endotoxin infusion $\mathrm{P}_{\mathrm{G}}=0.001, \mathrm{P}_{\mathrm{T}}=0.5, \mathrm{P}_{\mathrm{TXC}}=0.8$.

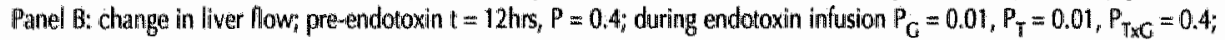
post-endotoxin infusion $\mathrm{P}_{\mathrm{C}}=0.3 \mathrm{P}_{\mathrm{T}}=0.001, \mathrm{P}_{\mathrm{TXC}}=1.0$.

Panel $C$ c change in bepatic antery flow, pre-endotoxin $t=12 \mathrm{hrs}, P=0.1_{\text {; }}$ during endotoxin infusion $P_{G}=0.01, P_{T}=0.004$, $P_{\text {TXC }}=0.02 ;$ post-endotoxin infusion $P_{G}=0.3, P_{T}=0.03, P_{T X C}=0.7$.

Panel $D$ : change in NO flux PDV; pre-endlotoxin $t=12 \mathrm{hrs}, P=0.01$; during endotoxim infusion $P_{G}<0.0001, P_{T}=0.3$, $P_{\mathrm{TuC}}=0.001 ;$ post-endotoxin infusion $P_{\mathrm{G}}=0.07, \mathrm{P}_{\mathrm{T}}=1.0, \mathrm{P}_{\mathrm{TXG}}=0.3$

Panel $E$ : change in NO flux liver; pre-endatoxin $t=12 \mathrm{hrs}, P=0.6$; during endotoxin infusion $P_{G}=0.03, P_{T}=0.01$, $P_{T \times C}=0.6 ;$ post-endotoxin infusion $P_{C}=0.09, P_{T}=0.09, P_{T \times G}=0.3$.

Figure 3 Hepatosplanchnic flow and nitric oxide production during L-arginine and saline (controls) pre-treated hyperdynamic endotoxaemia 


\section{Oxygen transport}

Oxygen consumption in the PDV and liver did not change upon arginine supplementation (Table 2, 12hrs; $\mathrm{P}=0.2$ and 1.0 for PDV and liver, respectively), but increased during endotoxaemia in both groups (Table 2, 20 and $36 \mathrm{hrs} ; \mathbb{P}_{\mathrm{T}}=0.006$ and 0.005 , respectively). This increase peaked earlier and appeared transient in arginine-treated animals. After stopping LPS, oxygen consumption decreased again (Table 2, 60hrs; $P_{T \times G}=0.2$ and 0.5 , respectively). Concomitant with the increased flow through the PDV and liver during LPS infusion, $\mathrm{PrCO}_{2}$ decreased in arginine-treated animals, but not in controls (Table 2, 20 and 36hrs; $P_{C}=0.003$ ). After stopping the LPS infusion, the decreased $\mathrm{PrCO}_{2}$ values in arginine-supplemented animals returned to control levels (Table 2, 60hrs; $P_{T \times G}=0.8$ ).

\section{PDV metabolic parameters}

\section{Arginine}

Arginine supplementation caused a higher arginine uptake in the PDV (Table 3. $12 \mathrm{hrs} ; \mathrm{P}<0.0001)$. Furthermore, prior to the endotoxin infusion arginine disposal and production were 2 -fold higher in arginine-supplemented than in control animals, although these differences were not statistically significant (Table 3, $12 \mathrm{hrs} ; \mathrm{P}=0.3$ ). The increased arginine uptake in arginine-treated animals persisted upon LPS treatment (Table 3, 20 and 36hrs; $P_{C}=0.002$ ), this treatment having no effect itself $\left(\mathrm{P}_{\mathrm{TXG}}=1.0\right)$. Arginine disposal and production were lowered during the first 8hrs of LPS treatment (Table 3, 12 to 20hrs; $P_{\mathrm{TxC}}>0.2$ ), but increased several-fold during the next $18 \mathrm{~h}$, especially in control animals (Table 3,36 until $60 \mathrm{hrs} ; P_{T \times C}=0.06$ and $P_{T \times G}=0.02$ ). Discontinuation of LPS treatment changed the arginine flux across the PDV from net uptake to net release in arginine-treated animals, but not in controls (Table 3,60hrs; $\mathrm{P}_{\mathrm{TxG}}<0.0001$ ). In control animals, arginine disposal and production declined to low levels, whereas these parameters remained at pretreatment levels in arginine-treated animals (Table 3, 60hrs; $P_{G}=0.07$ and 0.05 , respectively; $P_{T \times G}=1.0$ ).

\section{NO}

The production of NO by the PDV was significantly higher in the arginine-treatment group than in the control treatment group (Figure 1, $12 \mathrm{hrs}$ ). During LPS infusion, NO production did not change in the control group, but was temporarily suppressed (at $8 \mathrm{hrs}$ in the arginine-treated group (Figure 2, 20 and $36 \mathrm{hrs;} \mathrm{P}_{\mathrm{G}}=<$ $\left.0.0001 ; P_{T \times C}=0.001\right)$. After stopping LPS, NO production increased in the control group and remained elevated in the arginine-treated group (Figure 2, 60hrs).

\section{Citrulline}

Arginine treatment by itself did not modify citrulline release from the PDV (Table $3,12 \mathrm{hrs}$ ), but increased citrulline disposal and production (Table 3, $12 \mathrm{hrs} ; \mathrm{P}=0.02$ and $P=0.04$ ). LPS treatment decreased citrulline release in the control group, but only after more than $8 \mathrm{hrs}$ of treatment (Table 3,20 and 36hrs; $P_{T_{x G}}=0.009$ ). 


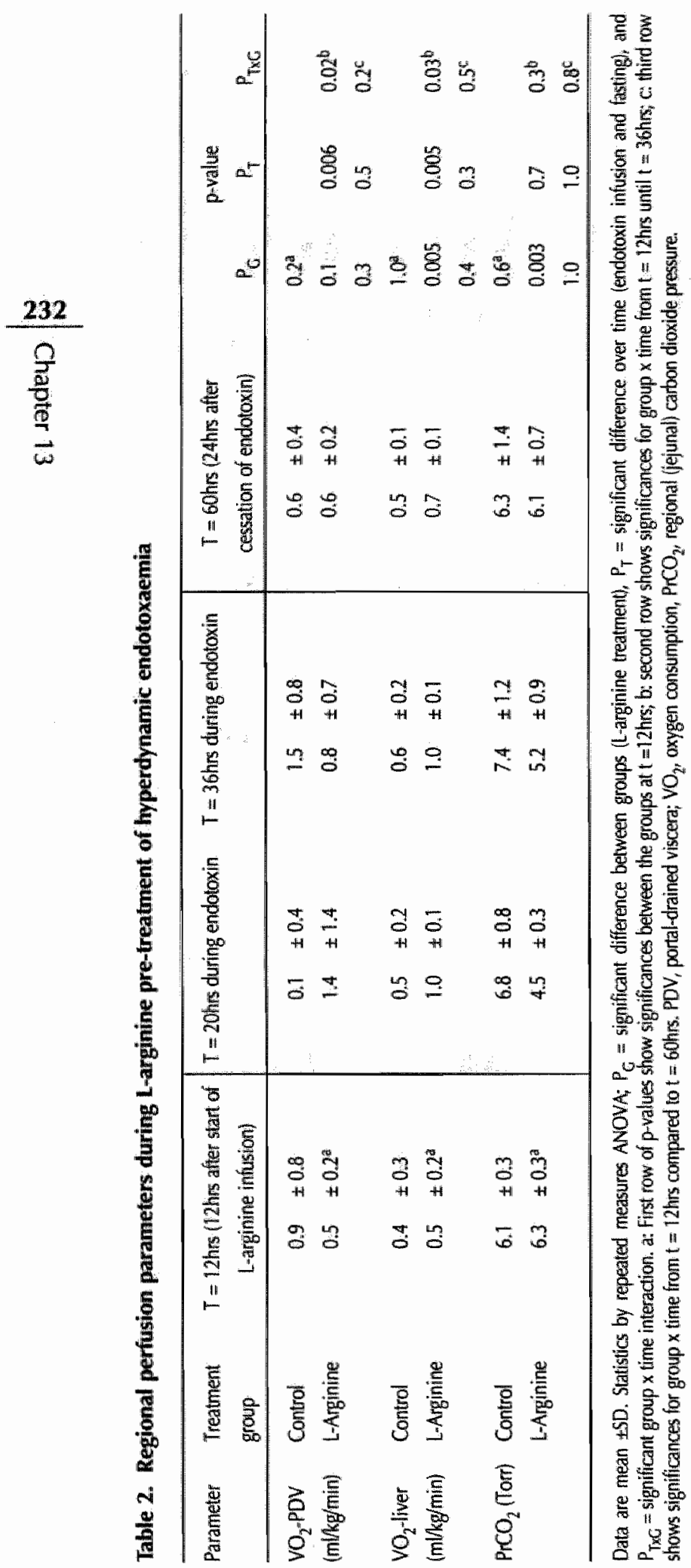




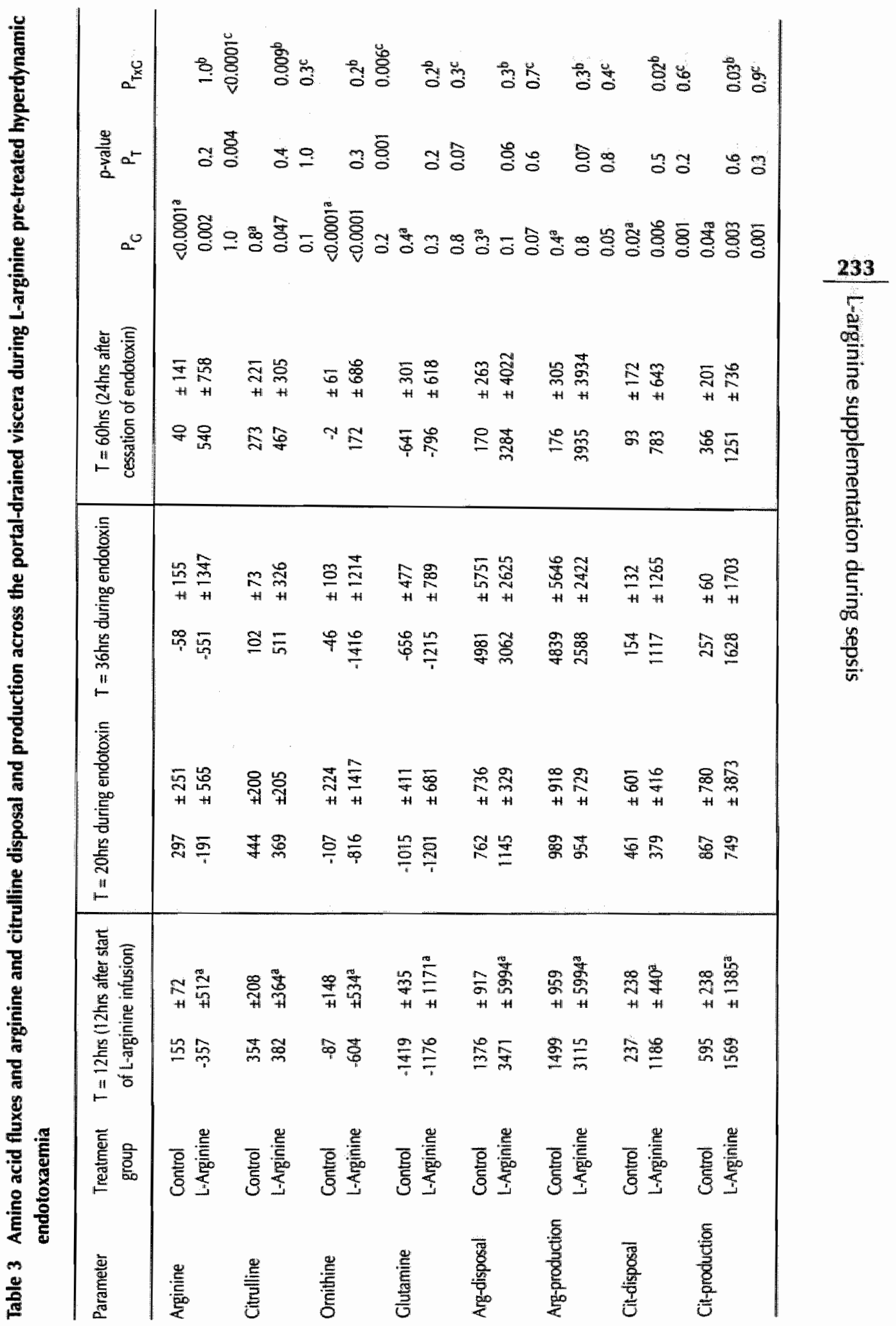


Treatment with LPS transiently increased citrulline disposal and production in control animals, but transiently decreased these parameters in arginine-supplemented animals. After stopping LPS infusion, citrulline release, disposal and production remained depressed in the control group (Table $3,60 \mathrm{hrs}$ ) and at pretreatment levels in arginine-treated animals (Table $3,60 \mathrm{hrs} ; \mathrm{P}_{\mathrm{G}}=0.001$ ).

\section{Ornithine}

A significantly higher net uptake of ornithine was observed in the arginine-sup234 plemented group (Table 3,12 thrs; $P_{C}=0.0001$ ). This difference between the control and arginine-supplemented group persisted during endotoxaemia (Table 3, 20 and 36hrs; $P_{T \times G}=0.2$ ). After stopping LPS, the release of ornithine in the arginine-treated group changed to a small uptake (Table $3,60 \mathrm{hrs} ; P_{T \times G}<0.006$ ).

\section{Glutamine}

Glutamine was taken up by the PDV in both study groups, but decreased after 8 hrs of endotoxin treatment in the control group. After stopping LPS, the suppressed glutamine uptake persisted at least $24 \mathrm{hrs}$ in the control group and became evident in the arginine-treated group (Table 3, 60hrs: $\mathrm{P}_{\mathrm{T}}=0.007$ )

\section{Hepatic metabolic parameters}

\section{Arginine}

The arginine net uptake in the liver was significantly higher in animals receiving supplemental arginine than in control animals (Table 4, 12hrs; $P_{C}=0.009$ ). Arginine disposal by the liver was not affected by arginine infusion, but arginine production tended to be higher (Table $4,12 \mathrm{hrs} ; \mathrm{P}=0.06$ ). LPS infusion increased arginine uptake in both control and arginine-supplemented animals, but in the latter group during the first $8 \mathrm{hrs}$ only (Table 4,20 and $36 \mathrm{hrs} ; \mathrm{P}<0.05$ ). Arginine disposal and production were transiently suppressed during the first $8 \mathrm{hrs}$ of LPS infusion in the control group, whereas these parameters were elevated in the arginine-treated group, especially after the first 8hrs of LPS treatment (Table 4, 20 and $36 \mathrm{hrs} ; \mathrm{P}_{\mathrm{T} \times \mathrm{C}}<0.05$ ). After stopping LPS infusion, arginine uptake returned to pretreatment values in both groups (Table 4,60hrs). Arginine disposal and production remained unchanged in the control group, but increased further in the arginine-treated group (Table 4, 60hrs; $\mathrm{P}_{\mathrm{TXC}}=0.01$ and $\mathrm{P}_{\mathrm{TxC}}=0.002$ ).

\section{NO}

Arginine treatment did not affect liver NO production (Figure 2B, 12hrs). Upon LPS administration, NO production significantly increased in both groups of animals (Figure 2B, 8 and 20hrs; $P_{G}=0.03$ ). In the arginine-treated group, this effect was most pronounced during the first 8 hrs of LPS treatment and appeared transient. After stopping LPS infusion, liver NO production returned to pretreatment values in the arginine-treated group, but remained elevated in the control group (Fig. 2B, 60hrs; $P_{G}=0.09$ ). 


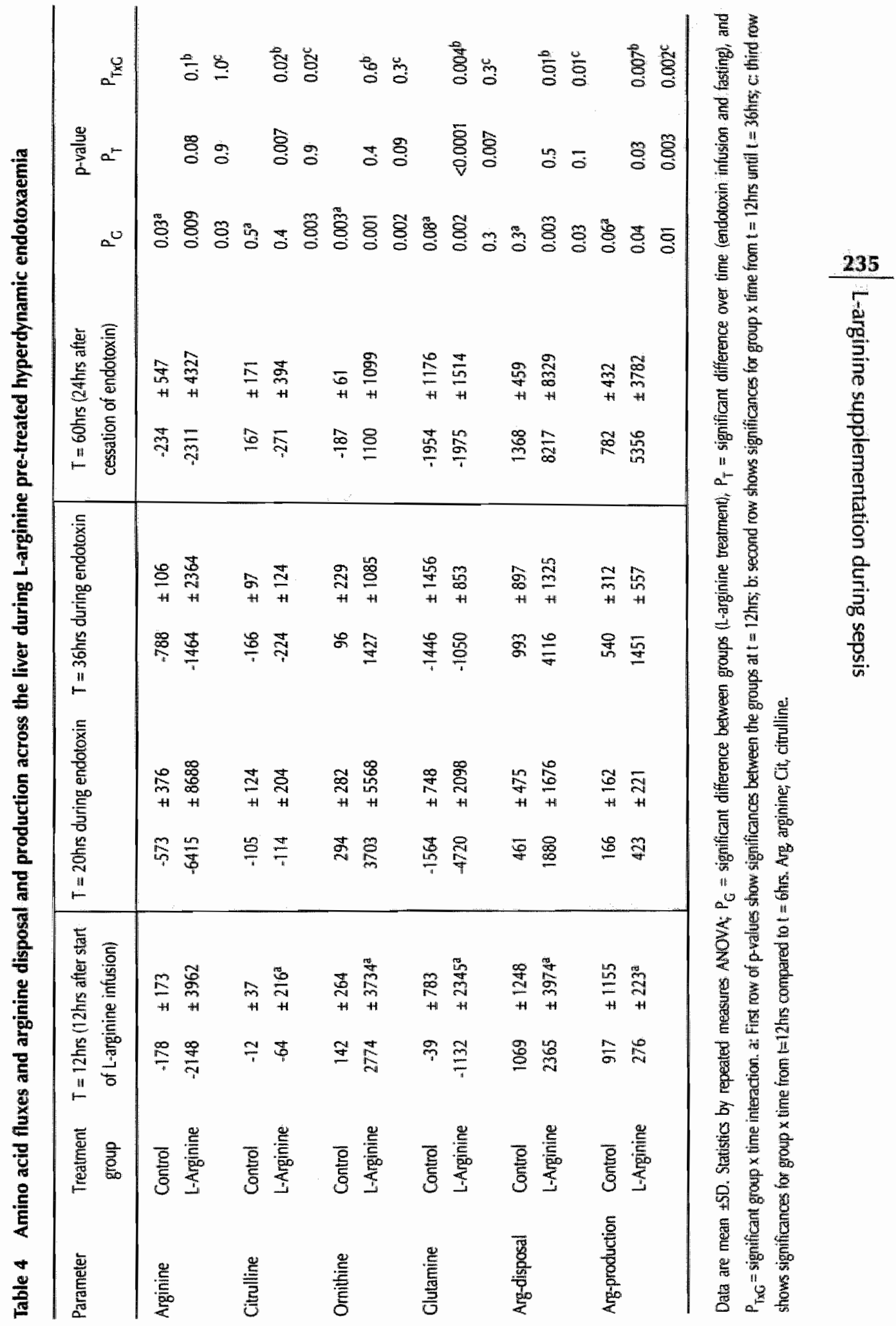




\section{Citrulline}

Citrulline uptake was similar in control and arginine-treated animals (Table 4, $12 \mathrm{hrs}$ ). LPS treatment increased liver citrulline uptake in arginine-treated animals, but after 8 hrs only (Table 4, 20 and 36hrs; $P_{T \times C}=0.02$ ).

\section{Ornithine}

The net release of ornithine from the liver was significantly higher in the arginine-treated than in control animals (Table 4, $12 \mathrm{hrs} ; \mathbb{P}_{G}=0.001$ ). During the first 8 hrs after LPS administration, ornithine release remained unchanged in both groups (Table 4, 20hrs; $\mathrm{P}_{\mathrm{T}}=0.2$ ), but during the next 16hrs, it decreased in both groups (Table $4,36 \mathrm{hrs} ; \mathrm{P}_{\mathrm{T}}>0.2$ ). After discontinuing LPS, ornithine uptake remained depressed in the arginine-treated group and even changed into a release in the control group (Table 4, 60hrs; $P_{G}=0.002$ ).

\section{Glutamine}

The net uptake of glutamine was significantly higher in the arginine than in the control group (Table 4, $12 \mathrm{hrs} ; \mathbb{P}_{\mathrm{G}}=0.002$ ). During the endotoxin infusion, glutamine uptake by the liver increased significantly, but in the arginine-treated group during the first 8hrs only (Table 4, 20 and $36 \mathrm{hrs;} P_{\mathrm{TXG}}=0.004$ ). After discontinuing LPS, glutamine uptake remained elevated in the control group (Table 4, 60hrs; $P_{\mathrm{T}}=0.007$ ).

\section{DISCUSSION}

We hypothesised that under conditions of acute and prolonged stress, such as endotoxin infusion, pre-treatment with arginine would improve perfusion and metabolism in the hepatosplanchnic area. The main conclusions from this study were that arginine supplementation per se did not affect whole-body cardiovascular parameters, but increased hepatosplanchnic perfusion; that arginine supplementation decreased peripheral resistance, increased cardiac output, and prevented an increase in PAP and glycolytic energy production during exposure to LPS; and that these beneficial effects were accompanied by and could probably be attributed to an increased NO synthesis at the whole-body level and in the liver, together with a temporarily decreased NO synthesis in the PDV.

Our porcine endotoxaemia model is characterised by a reduced mean arterial pressure, an increased cardiac index, and a decreased systemic vascular resistance. The increase in cardiac index and the decrease in pulmonary arterial pressure during endotoxaemia in arginine-supplemented animals suggest the development of a moderate systemic vasodilatation that does not lead to systemic hypotension. In septic patients, an intravenous bolus of arginine $(200 \mathrm{mg} / \mathrm{kg})$ induced only a brief (10-15 min) hypotension (13). These and our data therefore do not support earlier concerns that arginine supplementation may aggravate sepsis-induced hypotension. 
In control animals, arginine supplementation increased the flow through the PDV with approx. $35 \%$, but did not affect global perfusion or flow in the hepatic artery (Table 1). The effect of LPS on perfusion was virtually absent in the PDV and became manifest only slowly, that is after more than 8 hrs of exposure, in the cardiac index and hepatic artery. Arginine supplementation increased blood flow in all vessel beds. These data show that the flow in the systemic circulation, PDV and liver under basal conditions is less than $50 \%$ of the maximum perfusion and that, with the exception of the gut, arginine can decrease the vascular resistance and, hence, increase perfusion only if, in addition, inflammatory mediators are present as co-factors. Perhaps, such co-factors are constitutively produced in the gut.

Under baseline conditions whole-body arginine appearance depends on dietary intake, endogenous protein degradation and de novo synthesis. During arginine supplementation, whole-body arginine appearance increased 3 -fold in our study, as was reported earlier (7). The finding that this increased whole-body arginine appearance was not accompanied by an increase in whole-body de novo arginine synthesis, is in agreement with what is known for the regulation of arginine homeostasis. The kidneys play a major role in arginine homeostasis (14) by synthesising arginine from citrulline (15) that, in turn, is formed in the intestine from glutamine and proline (16). Although the kidney is capable of upregulating the arginine-synthesising enzymes argininosuccinate synthetase and argininosuccinate lyase in response to starvation and low arginine levels (17) and although de novo arginine synthesis in the kidney responds to changes in circulating levels of citrulline (18), the rate of de novo synthesis of arginine is not altered by increased or decreased circulating arginine levels $(18,19)$.

The extra appearance of arginine in arginine-supplemented animals represents only $25 \%$ of the arginine that was supplied, implying that the rest was metabolised. The substantial increase in arterial ornithine concentration in the arginine-supplemented animals (from $51 \mu \mathrm{M}$ to $256 \mu \mathrm{M}$ comparing control and arginine-supplemented animals at baseline) suggests that an increased flux through arginase is mainly responsible for this disposal of arginine. Somewhat to our surprise, the liver accounted for approx. $20 \%$ of the conversion of arginine into ornithine. The high $\mathrm{kM}$ arginine transporter in the liver probably explains the contribution of the liver to arginine catabolism (20).

LPS infusion increased whole-body arginine appearance 2 -fold, with a lag time of approx $8 \mathrm{~h}$, in agreement with earlier reports $(21,22)$. The decreased arterial arginine levels (from $79 \mu \mathrm{mol} / /$ to $64 \mu \mathrm{mol} / \mathrm{l}$ ) indicate that the increased appearance of arginine does not meet the increased disposal (7). This interpretation is supported by a pilot study, in which we showed that, compared to critically ill control patients, septic patients had reduced arginine levels associated with decreased whole-body arginine de novo synthesis rates (23). Under stress conditions such as trauma and sepsis, muscle protein catabolism becomes an important source of circulating arginine (1). Nevertheless, circulating arginine levels declined in endotoxaemic pigs (7). Arginine supplementation seems to prevent the increased disposal of arginine during sepsis (10). 
Arginine supplementation increases whole-body and visceral NO synthesis, but not liver NO synthesis, indicating that whole-body and intestinal NO production depend on arginine availability, whereas arginine availability is apparently not rate-determining in the liver until LPS induces NOS2 expression. Arginine also increases whole-body NO production in healthy human subjects (24). LPS alone does not increase whole-body and intestinal NO synthesis, perhaps due to a balance between NOS2 induction and lower circulating arginine levels. It does, however, decrease the arginine-induced increase in whole-body NO 238 synthesis and temporarily also that in the PDV, possibly due to down-regulation of NOS1 and -3. Furthermore, LPS increases liver NO production 2-fold, to levels similar to that measured constitutively in the intestine. As we suggested when discussing the effects of arginine on blood flow, this finding is compatible with a constitutively present low inflammatory state in the intestine, which is also suggested by the constitutive expression of NOS2 in enterocytes $(25,26)$. In the arginine-supplemented group, NO production was further enhanced, but only temporarily.

The apparently complementary behaviour of liver and intestine with respect to NO synthesis appears to be reflected in organ blood flow. The flow through the PDV did not differ between arginine-supplemented and control animals at $8 \mathrm{~h}$ after LPS, but was higher in arginine-supplemented animals at all other time-points. In contrast, the flow through the hepatic artery responded very quickly and was already 2 -fold increased at $8 \mathrm{~h}$ after LPS in arginine-supplemented, but not in control animals. The $\mathrm{Cl}$ showed, more weakly, the same response as the hepatic artery. Although we cannot explain these findings, we also note that NO production adapts more slowly to LPS in the entire body and the intestine than in the liver. Possibly the higher expression of Toll-like receptors in the liver compared to the intestine is responsible for these differences in organ behaviour (27). The finding that blood flow in the PDV is more sensitive to circulating arginine than to LPS may explain the relative failure of selective NOS2 inhibitors to affect splanchnic organ perfusion during endotoxaemia. Upregulation of splanchnic perfusion during prolonged endotoxemia may reflect the further upregulation of NOS expression $(6,7,28)$.

After cessation of LPS administration, whole-body and liver NO production and blood flow returned more quickly to basal rates than in the control group, supporting the interpretation that arginine supplementation partly suppresses the inflammatory response. Overall, these data suggest that administration of extra arginine appears to be an attractive treatment modality for serious, but elective operative interventions. Our earlier studies indicate that, when inflammation is already established, a brief treatment with a short-acting selective NOS2 inhibitor to treat the hypotensive episode may be considered (6). 


\section{REFERENCES}

1. Bruins $M$ ), Lamers WH, Meijer Al, Soeters PB, Deutz NE. In vivo measurement of nitric oxide production in porcine gut, liver and muscle during hyperdynamic endotoxaemia. $\mathrm{Br}$ I Pharmacol 2002; 137(8):1225-36.

2. Freund $\mathrm{H}$, Atamian S, Holroyde J, Fischer JE. Plasma amino acids as predictors of the severity and outcome of sepsis. Ann Surg 1979;190:571-6.

3. Lorente JA, Delgado MA, Tejedor $C$, et al. Modulation of systemic hemodynamics by exogenous L-arginine in normal and bacteremic sheep. Crit Care Med 1999;27:2474-9.

4. Scott JA, Metha S, Duggan M, Bihari A, McCormack DG. Functional inhibition of constitutive nitric oxide synthase in a rat model of sepsis. Am J Respir Crit Care Med 2002;165(1 0):1426-32.

5. Kirkeboen KA, Strand $\varnothing A$. The role of nitric oxide in sepsis--an overview. Acta Anaesthesiol.Scand 1999;43(3):275-88.

6. Poeze $M$, Bruins MI, Vriens I, Ramsay $G$, Deutz NEP. Selective iNOS inhibition decreases in vivo NO production, but increases mortality during porcine endotoxaemia. Intensive Care Med 2001;27(Suppl1):S243

7. Bruins MJ, Soeters PB, Lamers WH, Meijer AJ, Deutz NE. L-arginine supplementation in hyperdynamic endotoxemic pigs: effect on nitric oxide synthesis by the different ongans. Crit Care Med 2002;30(3):508-17.

8. Ten Have GAM, Bost MCF, Suyk-Wierts JCAW, van den Bogaard AEJM, Deutz NEP. Simultaneous measurement of metabolic flux in portally-drained viscera, liver, spleen, kidney and hindquarter in the conscious pig. Lab Animals 1996;30:347-58.

9. Castillto L, Beaumier L, AjamiA, Young VR. Whole body nitric oxide synthesis in healthy mean determined from [15N]arginine-to-[15N]citrulline labeling. Proc Natl Acad Sol USA 1996;93: 11460-5.

10. Bruins $M$ J , Soeters $P B$, Lamers $W H$, Deutz NE. L-arginine supplementation in pigs decreases liver protein turnover and increases hindquarter protein turnover both during and after endotoxemia. Am J Clin Nutr 2002;75(6):1031-44.

11. Wolfe RR. Radioactive and stable isotope tracers in biomedicine. Principle and practice off kinetic analysis. 1. New York: Wiley-Liss 1992; p.49

12. van Eijk HM, Rooyakkers DR, Deutz NEP. Rapid routine determination of amino acids in plasma by high-performance liquid chromatography with a 2-3 microns Spherisorb ODS II column. I Chromatogr 1993:620(1):143-8.

13. Lorente $\rfloor A$, Landin $L$, De Pablo $R$, Renes $E$, Liste $D$. L-arginine pathway in the sepsis syndrome.Crit Care Med 1993;21(9):1287-95.

14. Jenkinson $C P$, Grody WW, Cederbaum SD. Comparative properties of arginases. Comp Biochem Physiol B Biochem Mol Bioll 1996;114(1):107-32.

15. Dhanakoti SN, Brosnan ME, Herzberg GR, Brosnan IT. Cellular and subcellular localization of enzymes of arginine metabolism in rat kidney. Biochem / 1992;282(Pt 2):369-75.

16. Windmueller $\mathrm{HC}$, Spaeth $\mathrm{AE}$. Source and fate of circulating citrulline. Am J Physiol 1981 241(6): E473-E480

17. Morris SM jr. Regulation of enzymes of the urea cycle and arginine metabolism. Ann Rev Nutr 2002;2:87-105.

18. Dhanakoti SN, Brosnan IT, Herzberg GR, Brosnan ME. Renal arginine synthesis: studies in vitro and in vivo. Am / Physiol 1990;259:E437-E442 
19. Hallemeesch MM, Soeters PB, Deutz. NE. Renall arginine and protein synthesis are increased during early endotoxemia in mice. Am I Physiol 2002;282(2):F316-F323

20. Espat N], Watkins KT, Lind DS, Weis JK, Copeland EM, Souba WW. Dietary modulation of amino acid transport in rat and human liver. I Surg Res 1996;63(1):263-8.

21. Saetre T, Hoiby EA, Aspelin T, Lermark G, Egeland T, Lyberg T. Aminoethyl-isothiourea, a nitric oxide synthase inhibitor and oxygen radical scavenger, improves survival and counteracts hemodynamic deterioration in a porcine model of streptococcal shock. Crit Care Med 2000;28(8);2697-706.

22. Orellana RA, O'Connor PM, Nguyen $H V$, Bush $\mid A_{r}$ Suryawan $A_{f}$ Thivierge MC, Fiorotto ML, Davis TA. Endotoxemia reduces skeletal muscle protein synthesis in neonates. Am I Physiol 2002;283(5):E909-E916.

23. Luiking $Y C$, Steens $L$, Poeze $M$, et al. Low plasma arginine concentration in septic patients is related to diminished de novo arginine production from citrulline. [Abstract] Clin Nutr 2003;22:S26

24. Castillo L, DeRojas TC, Chapman TE, Vogt J, Burke JF, Tannenbaum SR, Young VR. Splanchnic metabolism of dietary arginine in relation to nitric oxide synthesis in normal adult man. Proc Natl Acad Sci USA 1993;90:193-7.

25. M'Rabet-Touil H, Blachier F, Morel MT, Darcy-Vrillon B, Duee PH. Characterization and ontogenesis of nitric oxide synthase activity in pig enterocytes. FEBS Lett 1993;331:243-7.

26. Di Lorenzo MF, Krantis A. Altered nitric oxide production in the premature gut may increase susceptibility to intestinal damage in necrotizing enterocolitis. I Pediatr Surg 2001;36:700-5.

27. Backhed $F$, Hornef $M$. Toll-like receptor 4-mediated signaling by epithelial surfaces: necessity or threat? Microbes Infect 2003;5(11):951-9.

28. Saetre T, Gundersen $Y$, Thiemermanin $C_{v}$ Lilleaasen $P$, Aasen AO. Amino-isothiourea, a selective inhibitor of inducible nitric oxide synthase activity, improves liver circulation and oxygen metabolism in a porcine model of endotoxemia. Shock 1998;9(2):109-15. 
CHAPTER 14

Summary and general discussion 
Last year, a woman aged 40 years was operated upon at our hospital for dysplasia of a polyp in the sigmoid. The sigmoid resection which was carried out in this generally healthy patient was complicated, however, by the occurrence of a clinical sepsis condition with shock. Our first thought was that a leakage of the anastomosis was the main problem, but the findings at relaparotomy could not explain the severity of the condition. Although subsequent cultures were all negative for bactería, the patient died six days after relaparotomy with an overt and severe multiple organ failure.

This case highlights two important issues. Firstly, sepsis and multiple organ failure have a major impact on mortality and hospital resources. It has been estimated that every day, around 1,400 patients worldwide die at the ICU as a result of sepsis (1). The incidence of sepsis in the Netherlands has been estimated to be 13.6 per 1,000 hospital admissions (2) and $25-30 \%$ of all patients admitted to the ICU had or developed sepsis during their stay (3-5). Of these patients, an estimated 3,500 die each year due to sepsis. Secondly, despite recent advances in reducing mortality (6-8), our understanding of the pathophysiological mechanisms of this syndrome in humans is still limited. Despite the many etiological factors that have been discovered in animal and in vitro experiments, physicians caring for patients with sepsis and multiple organ failure face two problems when applying these pathophysiological theories in everyday practice: do such factors exist in humans and is this an important mechanism?

The research underlying the present thesis focused on an examination of two pathophysiological mechanisms hypothesised to be important in multiple organ failure: (a) haemodynamic disturbances and (b) the inflammatory responses seen in critically ill patients with sepsis and multiple organ failure. Specific aspects investigated include the importance of a disturbed hepatosplanchnic perfusion and inflammation. Evaluating the importance of disturbances in global and regional haemodynamic parameters and inflammation is important, since failure to recognise these disturbances puts the patients at increased risk of MOF. Moreover, one of our studies showed that a majority of physicians caring for critically ill patients recognise the difficulty as well as the importance of detecting occult thaemodynamic disturbances with currently available tools (9). The pathophysiological mechanisms of hypovolaemia in surgical patients and the hyperdynamic profile of septic patients are well known, but it remains difficult to recognise these abnormalities in individual patients. Several researchers have previously demonstrated that in the majority of cases, intensivists were unable to correctly predict a patient's haemodynamic profile $(10,11)$. In our study, both intensivists and other physicians caring for these patients did indeed acknowledge that they frequently miss a diagnosis of sepsis. One of the observations made in this study was that the physicians agreed that a more aggressive monitoring regimen in patients with organ failure is justified. This means that better monitoring tools are required for critically ill patients.

In view of these observations, we formulated several research questions for the studies reported on in this thesis:

1. Does monitoring the global haemodynamic profile predict postoperative outcome in high-risk surgical patients better than standard haemodynamic parameters? 
2. Does improvement of the global haemodynamic parameters reduce mortality in high-risk surgical patients?

3. Does monitoring regionall perfusion improve the prediction of morbidity and montality compared to using globall haemodynamic parameters in critically ill patients?

4. Is the measurement of D-lactate as a regional parameter suitable for the detection of intestinal ischaemia in high-risk surgical or critically ill patients?

5. Do pre-operatively decreased regional oxygenation and perfusion predict post-operative outcome in high-risk surgical patients?

6. Does an increased inflammatory response in the hepatosplanchnic area precede the development of organ fallure in high-risk surgical patients, and is decreased regional oxygenation in the intestinal mucosa associated with an increased inflammatory response in such patients?

7. Does modulation of the inflammatory response improve the perfusion and metabolic functioning of the hepatosplanchnic area in experimental sepsis and organ failure?

\section{Global haEmodynamic monitoring}

The thermodilution method, using a pulmonary artery floatation catheter (PAFC) and the central venous catheterisation methods are the most frequently used techniques of invasive monitoring in critically ill and high-risk surgical patients. However, several arguments justify the search for other monitoring techniques. Firstly, invasive techniques are associated with a number of serious complications (12). Siecondly, a number of studies have questioned the validity of measurements obtained using central venous and pulmonary artery catheters $(13,14)$. Although gross haemodynamic disturbances can be monitored adequately, measuring venous pressures is limited as a method for detecting occult haemodynamic disturbances (15).

The use of less invasive monitoring techniques, such as Doppler ultrasonography and bio-impedance, has been advocated to reduce catheter-related complications, but whether the predictive power of these techniques is better than the currently used monitoring techniques was until recently unknown. Our study used transoesophageal Doppler and found that it was possible to predict with good accuracy the development of post-operative complications in haemodynamically stable high-risk surgïcal patients (positive likelihood ratio $=4.9$ ) (chapter 4 ). A failure to recognise haemodynamic deterioration using standard monitoring techniques or a failure to improve the Doppler ultrasonography parameters by therapeutic interventions were likely to predict an unfavourable outcome. A number of studies have shown that correcting the abnormalities detected by transoesophageal Doppler ultrasonography can reduce the morbidity rate 
in high-risk surgery $(16,17)$. Other authors have suggested that oesophageal Doppler sonography should replace the Swan-Ganz catheter in high-risk surgical patients (18), although no randomised clinical trial comparing the two methods has as yet been performed. The reason for this improved ability to detect occult haemodynamic abnormalities seems to be related to the type of parameter studied. Clinicians using standard monitoring and Swan-Ganz monitoring techniques usually monitor pressure-related parameters. Our study, using transoesophageal Doppler ultrasonography, indicated that monitoring volume-related parameters improves the prediction of outcome. Moreover, global volume-related parameters using double-dye dilution techniques predicted mortality among ICU patients better than pressure-related parameters using standard and thermodilution techniques (chapter 7).

It is likely that changes in perfusion or volume status contribute to the complication rate in surgical patients. Patients undergoing elective major abdominal surgery are starved for at least 4-10hrs to reduce the risk of aspiration. The diseases for which the patients are operated upon frequently cause weight loss, nausea or vomiting. Moreover, a considerable insensible fluid loss occurs during abdominal surgery. All these factors can contribute to an increased risk of hypoperfusion in the patient prior to, and during, surgery. This does not usually cause any change in blood pressure, because of compensatory vasoconstriction. In these circumstances, non-invasive monitoring techniques, such as transoesophageal Doppler ultrasonography, have a higher predictive value in detecting complications, since these are more closely related to abnormal volumes than abnormal pressures.

\section{Global haEmodynamic optimisation}

Uncorrected and occult global haemodynamic abnormalities are apparently related to an increased complication rate in surgical patients. In previous studies, haemodynamic optimisation techniques were used during surgery to improve outcome (19-21). Our study employed dopexamine in two doses to improve oxygen delivery to the tissues (chapter 5 ). It found that dopexamine was unable to decrease peri-operative mortality in the total study group. Results of a previous study showed improved survival using dopexamine in high-risk abdominal surgery (21). Similarly, Wilson et al. demonstrated decreased mortality and morbidity rates in high-risk surgical patients optimised with either dopexamine or noradrenaline (22).

The concept of beneficial effects of increased perioperative and postoperative oxygen delivery is based on both pathophysiologic and clinical reasoning. Surgery increases the metabolic demand, and an inability to respond to this by increasing cardiac output and thus oxygen dellivery predisposes the tissues to inadequate perfusion and subsequent organ dysfunction (23). The relevance of impaired 
physiologic reserves was shown in patients with a low aerobic threshold in preoperative exercise testing (24), who suffered increased postoperative morbidity and mortality. Most importantly, maintaining increased blood flow and oxygen delivery is more likely to be beneficial when used in a preventive fashion rather than after vital organ dysfunction has already been established. Dopexamine is a relatively new synthetic dopamine derivate and is a very potent beta ${ }_{2}$-agonist, a weak beta ${ }_{1}$-agonist and a dopaminergic agonist. Its effect is mainly one of vasodilatation and afterload reduction, rather than contractility improvement. Since its vasodilatory effects make it an interesting drug, due to its claimed effects on the circulation of the gut (25), dopexamine was used in all three of the above-mentioned optimisation studies. There were, however, differences in set-up between these studies. Our own study used a fixed dose of dopexamine, in contrast to the other two studies, in which the dopexamine dose was titrated to increase oxygen delivery. Moreover, the mean dopexamine doses in the studies by Boyd et al. and Wilson et al. was generally lower than those used in our study. Previous studies have indicated the importance of titrating the infusion rate of the vaso-active medication to the patient's individual needs (26). High-risk surgical patients with multiple co-morbidities do not tolerate inotropes in the same way as patients without these co-morbidities do when undergoing high-risk surgery. One study using high doses of dobutamine found increased mortality (27).

Another and perhaps more important factor underlying the difference in the outcome of the studies is baseline mortality. The two other studies using dopexamine found mortality in the control group to be around $20-30 \%$, which was reduced to below $10 \%$ in patients. undergoing optimisation strategies. Mortality in the placebo group in our study was $13 \%$, while patients using $0.5 \mu \mathrm{g} / \mathrm{kg} / \mathrm{min}$ dopexamine had a mortality of $5 \%$. This may imply that in populations having a low a priori risk of death, the effects of interventions will be less significant when using a comparable number of patients. A meta-analysis revealed that reduction in mortality was reported in those studies with a mortality rate in the placebo group of around $20 \%$. Overall, mortality in high-risk surgical patients was shown to be significantly reduced, from $15.1 \%$ to $6.5 \%$ (chapter 6 ). Post-hoc analysis in our study using dopexamine showed a significant reduction in mortality in patients undergoing emergency surgery, who had an a priori risk of death of $29 \%$.

Thus, some conditions must be met before peri-operative optimisation can be effective. Timing of the intervention seems to be essential (28).

Data on comparisons between various medications during optimisation strategies are relatively scarce. Wilson et al. compared the use of dopexamine and noradrenaline in high-risk surgical patients, and despite the apparently similar reduction in mortality, dopexamine reduced morbidity more than noradrenaline (22). This study indicates that more trials on the effects of various medications (or combinations of medications) are necessary. The importance of using adequate dosages of the various drugs has been argued before, and adequate patient selection is essential to achieve positive results when using optimisation techniques. 


\section{HEPATOSPLANCHNIC HAEMODYNAMIC MONITORING}

While the hepatosplanchnic organ system was previously not considered to play an important role during sepsis and organ failure, experimental data have provided strong evidence for a key role of haemodynamic and inflammatory splanchnic dysfunction during sepsis. Numerous studies have now indicated that abnormalities in the functioning of hepatosplanchnic organs can serve as a litmus test to detect occult haemodynamic abnormalities during sepsis and multiple organ failure $(29,30)$. Several techniques have recently been developed to detect these abnormalities. Our study (chapter 7 ) provided a prospective comparison of some of these techniques, evaluating both global and regional haemodynamic parameters. We found that monitoring hepatosplanichnic perfusion and functioning is superior to standard global monitoring techniques. Previously, Edouard et al. were able to demonstrate abnormal splanchnic perfusion, while global perfusion was within the normal range, in a study simulating hypovolaemia in healthy subjects by applying negative pressure on the lower extremities (29). Ivatury et al. found that mortality remained high in trauma patients when correction of global oxygen delivery was not accompanied by correction of gastric $\mathrm{pHi}$ (31).

A comparison between the area under the ROC curve for $\mathrm{pHi}$ found in a study by Maynard et al. and that found in our study reveals similar data (32). Interestingly, the significant differences between survivors and non-survivors in their study were found only on day 3 after admission. Although significant differences in splanchnic perfusion parameters between survivors and non-survivors were found in our study at admission, a significant difference in the predictive value was found after $24 \mathrm{hrs}$ and not directly at admission (chapter 7). Apparently, the measured disturbances in splanchnic perfusion and functioning become important after correction of parameters of global perfusion, due to the relative resistance of splanchnic perfusion abnormalities to resuscitation. During the early resuscitation period in critically ill patients, global perfusion disturbances are equally important predictors of outcome as disturbances in splanchnic perfusion.

In search of clinical parameters of disturbed splanchnic perfusion, D-lactate was investigated (chapter 8 and 9). The condition D-lactataemia or D-lactic acidosis was first described in humans with a short bowel syndrome (33). Subsequently, Murray et al. showed that patients with mesenteric thrombosis had increased D-lactate levels (34). Our study showed that increased D-lactate values can be found in patients with intestinal ischaemia following the repair of an acute abdominal aneurysm. D-lactate production occurs when carbohydrate malabsorption in the small bowel leads to increased delivery of carbohydrates to the colon with subsequent fermentation by colonic bacteria to L- and D-lactate. Only D-lactate accumulates, due to its slower metabolism. In contrast to what was previously thought, humans can metabolise D-lactate, probably by d-2-hydroxy-aciddehydrogenase, a mitochondrial enzyme, that converts D-lactate into pyruvate. The concentration of D-lactate in systemic blood samples, therefore represents a dysbalance between production and metabolism. This implies that increased levels of D-lactate can be found during conditions with non-absolute ischaemia. 
In a study by Sun et al., D-lactate was released during experimental conditions of non-absolute intestinal ischaemia, such as pancreatitis and burns (35). In another study, in pre-term infants, urinary D-lactate levels were increased temporarilly after birth (36). Our study attempted to relate D-lactate levels with the level of splanchnic oxygenation, as determined by tonometry, and found a significant correlation between the levels of $\mathrm{D}$-lactate and gastric $\mathrm{PrCO}_{2}$ in patients with severe sepsis.

Other techniques to monitor the hepatosplanchnic function during sepsis and multiple organ failure were also analysed. The technique of monitoring the intramucosal $\mathrm{PrCO}_{2}$ and $\mathrm{pHi}$ has previously received much attention, with a significant association being found between abnormal parameters of splanchnic tonometry and the outcome in a variety of patient populations. High-risk surgical patients undergoing major abdominal (37), vascular (38) and cardiac (39) surgery had increased morbidity and mortality rates when postoperative $\mathrm{PrCO}_{2}$ was high or $\mathrm{pHi}$ was low. Our data showed that $\mathrm{pHi}$ measured pre-operatively predicted morbidity and mortality in high-risk abdominal surgery (chapter 10). Apparently, it is not only the stress response to the surgical procedure and the reduced circulating volume induced by surgery that induces intramucosal acidosis, as had been demonstrated before, but some patients have a reduced splanchnic oxygenation from the start. This has important implications for clinical practice in high-risk surgical patients. Interestingly, patients who had an abnormal preoperative $\mathrm{pHi}$ that could not be corrected using fluids and vaso-active medications such as dopexamine had an increased risk of developing multiple organ failure. Kirton et al. showed that patients who achieved a normal pHi did not die of multiple organ failure (40). As mentioned above, Ivatury et al. showed that patients failing to improve a reduced gastric $\mathrm{pHi}$ were prone to die of their trauma-related multiple organ failure (31).

The inability of the vascular system to respond to interventions aimed at correcting the abnormal condition during sepsis, termed 'vasoplegia' or vasomotor dysfunction, is thought to play a crucial role in the development of organ failure $(41-45)$. The occurrence and extent of this vasoplegia is influenced by several factors. One of the key features of disturbed vascular reactivity during sepsis and organ failure is a dysregulated inflammatory response. Numerous experimental and clinical studies have indicated that modulation of this inflammatory response, whether using inhibitors or stimulators, influences the occurrence and extent of organ failure. This leads to the question whether this inflammatory response can also be detected in the hepatosplanchnic area in humans. Douzinas et al. found that in patients with overt organ failure due to sepsis, the hepatosplanchnic area is an important producer of inflammatory mediators, especially when these organs demonstrate clinical signs of failure (46). The questions we wanted to answer were: (a) does this regional inflammatory response occur in surgical patients and is it related to the development of multiple organ failure, and (b) is this inflammatory response related to other parameters of splanchnic dysfunction? In our studies we showed that prior to the development of organ failure in high-risk surgical patients, the hepatosplanchnic area displays an exaggerated inflammatory response (chapter 11 ). Whether this response 
is causally related to organ dysfunction in humans remains to be elucidated. The primary aim of the inflammatory response in the gut and liver is local control of a noxious stimulus. An overwhelming stimulus or failing compensatory mechanisms may cause an 'overflow' of inflammatory cytokines into the bloodstream. The finding that inhibition of inflammatory mediators selectively reduces the number of patients with multiple organ failure and also reduces its impact $(47)$ is indicative of such a causal relationship. Monitoring this regional inflammatory response would in theory be of use in high-risk surgical patients, but is technically difficult in practice. Further experiments could relate this regional hepatosplanchnic inflammatory response to more easily obtainable parameters. In our study, this increased inflammatory response coincided with both an increased overall flow in the hepatosplanchnic organs and a decreased gastric mucosal $\mathrm{CO}_{2}$ production. There is a clear rellationship between the inflammatory response and the extent of the splanchnic oxygenation disturbances. In clinical practice, hepatosplanchnic monitoring of high-risk surgical or critically ill patients may therefore not necessarilly require measurement of hepatosplanchnic inflammatory markers.

\section{HEPATOSPLANCHNIC HAEMODYNAMIC OPTIMISATION}

If hepatosplanchnic perfusion and inflammation play an important role in the development of organ failure after surgery and during sepsis, one may wonder whether it is possible to influence this reaction specifically in this area, thereby influencing the occurrence or impact of multiple organ failure? One of the key mediators of the inflammatory response seems to be nitric oxide, an oxygen radical which is produced in large quantities during sepsis. Produced in physilological quantities, NO, which is formed by the endogenous NO synthase (NOS I and 3), is a crucial mediator involved in proper organ functioning, involved for instance in the maintenance of gut mucosal blood flow. Enhanced formation of $\mathrm{NO}$ by the inducible form of NOS (NOS2) contributes significantly to the clinical features of sepsis, such as hypotension and increased vascular permeability. Several previous studies have investigated the effects of modulation of the nitric oxide metabolism on the hepatosplanchnic function. Increased levels of NO have been associated with increased ethanol-induced gastric mucosal damage, partly induced by changes in gastric mucosal blood flow (48). Other studies, however, indicate that both selective and non-selective inhibition of NO production is detrimental during sepsis (49-52) by reducing hepatosplanchnic perfusion and functioning. Thus, modulating NO production could provide a model to study the effects of influencing the interaction between perfusion and inflammation in the hepatosplanchnic area.

In our experimental studies we found that inhibiting nitric oxide production reduced hepatosplanchnic metabolic function and increased mortality during 
prolonged endotoxaemia (chapter 12). Several hypotheses can be formulated why inhibiting NO synthesis is detrimental during sepsis. One hypothesis is that sepsis leads to NOS2-induced NO synthesis to increase flow by vasodilatation in order to compensate for the increased metabolic demands. NOS2 inhibition, however, led to flow in the portal-drained viscera being increased, although only temporarily. Another explanation may be related to the sepsis-induced reduction in $\mathrm{NOSI}+3$ expression. This reduces the production of endogenously synthesised NO which is necessary to maintain normal flow. We hypothesised that this reduced NOS1 +3 -induced NO production is compensated by activation of NOS2. Although this increased production in itself may have detrimental effects (hypotension, vascular hyporeactivity), these are only of limited duration. Therefore, inhibiting the NOS2-induced NO production would suppress this compensatory mechanism and could thereby have detrimental influences on the NO-driven metabolism. In our second experimental study, infusion of L-arginine as an NO donor improved hepatosplanchnic metabolism and perfusion (chapter 13). Results from a literature study indicate that arginine supplementation is beneficial in a number of critically illnesses (53). Arginine depletion during surgery and ischaemia/reperfusion processes is associated with increased risk of infection and multiple organ failure, and treatment with arginine may alter this course. In a pilot study among patients with septic shock, we showed that whole-body arginine de-novo synthesis was reduced compared to that in control ICU patients, leading to reduced systemic arginine levels (54). Interestingly, these patients did not have an increased NO production compared to ICU controls (although NO synthesis was increased compared to healthy controls). Arginine synthesis tended to be lower in the septic shock patients. These data do indeed suggest arginine depletion in patients with septic shock. Our pig model showed that even during conditions with an apparent excess of nitric oxide production, arginine supplementation may be beneficial.

The conclusions from the studies discussed in this thesis emphasise the importance of monitoring global haemodynamic perfusion and of using haemodynamic optimisation techniques to prevent multiple organ failure in high-risk surgical patients. When monitoring global perfusion, global volume-related parameters should be used in preference to pressure-related parameters. Moreover, monitoring global perfusion does not seem to be sufficient in patients with established multiple organ failure. Other techniques, which can monitor the hepatosplanchnic organs, are necessary to recognise occult perfusion and inflammatory disturbances. These disturbances in hepatosplanchnic organ perfusion and functioning play an important role in the development and progression of multiple organ failure. Finally, experiments modulating nitric oxide, as a key player in perfusion and metabolism during sepsis indicated that supplementing arginine, as a precursor of nitric oxide, is beneficial to hepatosplanchnic perfusion and metabolism. 


\section{CONCLUDING REMARKS AND FUTURE RESEARCH}

An important question emerging from this thesis is whether interventions directed by parameters of splanchnic function influence the outcome in patients with sepsis and multiple organ failure. Some evidence in this thesis indicates that improved hepatosplanchnic perfusion and metabolism may alter outcome during sepsis and organ failure.

Previously, some clinical studies in patients with sepsis and multiple organ failure have been performed which aimed specifically at an improved hepatosplanchnic function. Gutierrez et al. demonstrated improved survival, but only in the group with a normal pHi (55). These findings suggest that optimisation techniques have an effect on mortality in patients with early sepsis (normal pHi), but that they have no effects in patients with severe sepsis (low pHi). These data are in line with the results reported by Rivers et al. (6). In contrast, Ivatury found no improved survival, despite an increase in gastric pHli (56). Mortality decreased from $29 \%$ to $8 \%$ in gastric pHi- directed therapy in a study by Barquist ett all., although this difference was not significant, probably due to the small number of patients (57). In any case, the number of patients with multiple organ failure decreased significantly. Spies et al. found no difference in overall mortality, but found a decreased mortality rate in patients who responded to the treatment with an increase in $\mathrm{pHi}$ (58). Finally, a study by Gomersall et al. found no difference in mortality, but their treatment did not result in a difference in pHi (59).

Thus, some questions can be formulated for future studies. The first question is which parameter best reflects the perfusion abnormalities in the hepatosplanchnic organs during sepsis and organ failure. Is gastric tonometry better than measuring total hepatosplanchnic blood flow or better than measuring $D$-lactate values in the blood? To solve this question we must know the importance of the heterogeneity of splanchnic perfusion during sepsis as a confounder of the parameter we want to validate. Is measuring the function of the entire splanchnic area (not taking local heterogeneity into account) as predictive as measuring the function of a local area (intramucosal $\mathrm{pHi}$ or Doppler flowmetry)? And if the latter technique is better, at what site should we measure function?

After these questions have been answered, the next question would be what intervention has a beneficial influence on this parameter in patients with sepsis and organ failure? Does modulating the arginine-nitric oxide metabolism improve splanchnic perfusion in patients with sepsis and multiple organ failure? Currently, we are performing this study on our intensive care unit. Subsequently, is this modulation related to an improved outcome in patients with sepsis and multiple organ failure who are resistant to other therapies?

It is clear that a considerable number of questions remain to be answered. The research underlying the present thesis provided evidence that, as experimental studies had suggested, haemodynamic and inflammatory disturbances are important in the hepatosplanchnic dysfunction during sepsis and organ failure.

Our studies of global haemodynamic monitoring have demonstrated that monitoring global volume-related parameters can detect occult haemodynamic distur- 
bances earlier and more accurately than the parameters we are currently using. Haemodynamic optimisation reduced mortality in high-risk surgical patients when used in a preventive fashion and titrated to the individual patient. The studies on hepatosplanchnic monitoring showed that measuring the perfusion and functioning of the hepatosplanchnic organs improves the ability to detect occult haemodynamic disturbances in patients with sepsis and multiple organ failure. 


\section{REFERENCES}

1. Grotz $M R$, Ding J, Guo W, Huang $Q$, Deitch EA. Comparison of plasma cytokine levels in rats subjected to superior mesenteric artery occlusion or hemorrhagic shock. Shock 1995;3(5);362-8.

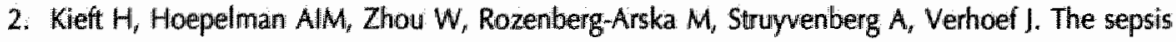
syndrome in a Dutch University Hospital. Arch Intern Med 1993;153:2241-7.

3. Salvo I, Caun de W, Musicco M, Langer M, Paidena R, Wolfler A, Mantani C, Magni $E$. The sepsis study group. The Italian SEPSIS study: preliminary results on the incidence and evolution of SIRS, sepsis, severe sepsis, and septic shock. Intensive Care Med 1995;21:S244-S249

4. Smäll $N_{s}$ Messiah $A_{x}$ Edouard $A_{x}$ Descorps-Declere $A$, Duranteau l, Vigue $B$, Momoz $O$, Samii $K$ Role of systemic inflammatory response syndrome and infection in the occurrence of early multiple organ dysfunction syndrome following severe trauma. Intensive Care Med 1995;21:813-6.

5. Rangel-Frausto MS, Pittet $D$, Costigan $M_{r}$ Hwang $T$, Davis CS, Wenzel RP. The natural history of the systemic inflammatory response syndrome. A prospective study. JAMA 1995;273(2):117-23.

6. Riwers E, Nguyen B, Havstad S, Ressler I Muzzin A, Knoblich B, Peterson E, Tomlanovich $M_{*}$ Early Goal-Directed Therapy Collaborative Group. Early goal-directed therapy in the treatment of severe sepsis and septic shock. New Eng J Med 2001;345 (19):1368-77.

7. Bernard $\mathrm{GR}$, Vincent J-L, Laterre $\mathrm{PF}$, LaRosa SP, Dhainaut JF, Lopez-Rodriguez A, Steingrub JS, Garber GE, Helterbrand JD, Ely EW, et al. Efficacy and safety of recombinant human activated protein C for severe sepsis. New Eng J Med 2001; 344(10):759-62.

8. van den Berghe $G_{v}$ 'Wouters $P$, Weekers $F$, Verwaest $C$, Bruyninckx $F$, Schetz $M$, Vlasselaers $D$, Ferdinande $P$, Lauwers P, Bouillon R. Intensive insulin therapy in the critically ill patients. New Eng I Med 2001;345(19):1359-67.

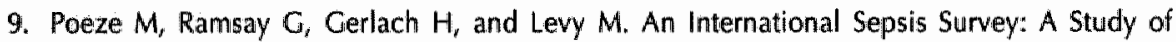
Doctors' Knowledge and Perception about Sepsis. Forthcoming

10. Connors AF, McCaffree DR, Gray BA. Evaluation of right-heart catheterization in the critically ill patient without acute myocardial infarction. New Eng I Med 1983;308(5):263-7.

11. Fein AM, Goldberg SK, Walkenstein MD, Dershaw B, Braitman L, Lippmann ML. Is pulmonary artery catheterization necessary for the diagnosis of pulmonary edema? Am Rev Resp Dis 1984;129;6):1006-9.

12. Putterman CE. The Swan-Ganz catheter: A decade of hemodynamic monitoring. I Crit Care $1989 ; 4: 127$

13. Wong $\mathrm{DH}_{\text {, Tremper }} \mathrm{KK}$, Stemmer EA, O'Connor $\mathrm{D}$, Wilbur $\mathrm{S}$, Zaccari \, Reeves $\mathrm{C}$, Weidoff $\mathrm{P}$, Trujllo RJ. Noninwasive cardiac output: simultaneous comparison of two different methods with thermodilution. Anaesthesiology 1990;72:784-92.

14. Pulmonary Artery Catheter Consensus Conference Participants. Pulmonary artery catheter consensus conference: consensus statement. Crit Care Med 1997;25(6):910-525.

15. Bindels $A$, van der Hoeven $J G$, Graafland $A D$, Koning J, Meinders $A E$. Relationships between volume and pressure measurements and stroke volume in critically ill patients. Crit Care $2000 ; 4(3): 193-9$

16. Sinclair $S_{i}$ James $S$, Singer $M$. Intraoperative intravascular volume optimisation and length of hospital stay after repair of proximal femur fracture: randomised controlled trial. BMI 1997;315:909-12.

17. Mythen $M G$, Webb AR. Perioperative plasma volume expansion reduces the incidence of gut mucosal hypoperfusion during cardiac surgery. Arch Surg 1995;130(4):423-9. 
18. Dabrowski GP, Steinberg SM, Ferrara J1, Flint LM. A critical assessment of endpoints of shock resuscitation. Surg Clin North Am 2002;80(3):825-44.

19. Shoemaker WC, Appel PL, Kram HB, Waxman MD, Lee T-S. Prospective trial of supranormal values of survivors as therapeutic goals in high-risk surgical patients. Chest 1988;94(6):1176-86.

20. Curran JE, Grounds RM. Ward versus intensive care management of high-risk surgical patients. Br) Surg 1998;85:956-61.

21. Boyd O, Grounds RM, Bennett ED. A randomized clinical trial of the effect of deliberate perioperative increase of oxygen delivery on mortality in high-risk surgical patients. JAMA 1 993;270:2699-707.

22. Willson J, Woods I, Fawcett J, Whall R, Dibb W, Morris $C_{s}$ McManus E. Reducing the risk of major elective surgery: randomised controlled trial of preoperative optimisation of oxygen delivery. BMJ. 1999;318(7191):1099-103.

23. Cannon $J G$, Tompkins $\mathbb{R} G$, Gelfand $J A_{f}$ et al.. Circulating interleukin- 1 and tumor necrosis factor in septic shock and experimental endotoxin fever. I Infect Dis 1990;161:79-84.

24. Sturk $A$, wan Deventer $S \mathrm{~J} \mathrm{H}$, Wortel $\mathrm{CH}$, et al. Detection and clinical relevance of human endatoxemia. Z Med Lab Diagn 1990;31:147-58.

25. Chintala MS, Moore RJ, Lokhandwala MF, Jandyala BS. Evaluation of the effects of dopexamine, a novel DA1 receptor and b2-adrenoreceptor agonist, on cardiac function and splanchnic circulation in a canine model of hemorrhagic shock. Arch Pharmacol 1993;347:296-300.

26. Bone RC. The pathogenesis of sepsis. Ann Intern.Med 1991;11:457-69.

27. Hayes MA, Timmins AC, Yau EHS, Palazzo M, Hinds Cl, Watson D. Elevation of systemic oxygen delivery in the treatment of critically ill patients. New Eng I Medi 1994;330(24):1717-22.

28. Boyd O, Hayes M. The oxygen traill: the goal. Br Med Bull 1999;55(1):125-39.

29. Edouard AR, Dergremont A-C, Duranteau J, Pussard E, Berdeaux A, Samii K. Heterogeneous regional vascular responses to simulated transient hypovolemia in man. Intensive Care Med 1994;20:414-20.

30. Dahn MS, Lange $P$, Lobdell $K$, Hans $B$, Jacobs LA, Mitchell RA. Splanchnic and total body oxygen consumption differences in septic and injured patients. Surgery 1987;101(1):69-80.

31. Ivatury RR, Simon Rl, Havriliak D, Garcia C, Greenbarg I, Stahl WM. Gastric mucosal pH and oxygen delivery and axygen consumption indices in the assessment of adecpuacy of resuscitation after trauma: a prospective, randomised study. J Trauma 1995;39(1):128-36.

32. Maynard ND, Bihari DI, Dalton RN, Beale R, Smithies MN, Mason RC. Liver function and splanchnic ischemia in critically ill patients. Chest 1997;111(1):180-7.

33. Oh MS, Phelps KR, Traube M, Barbosa-Saldivar IL, Boxhill C, Carroll HJ. D-lactic acidosis in a man with the short-bowel syndrome. New Eng \& Med 1979;301(5):249-52.

34. Murray M), Gonze MD, Nowak LR, Cobb CF. Serum D(-)-lactate levels as an aid to diagnosing acute intestinal ischemia. Am I Surg 1994;167(5):575-8.

35. Sun $X Q$, Fu $X B_{x}$ Zhang $R$, Lu $Y$, Deng $Q$, liang $X \mathrm{C}$, Sheng $Z Y$. Relationship between plasma $D(-)-$ lactate and intestinal damage after severe injuries in rats. World J Gastroenterol $2001 ; 7(4) ; 555-8$.

36. Rouwet $E$, Heineman $E$, Ter Riet $G_{x}$ Ramsay $G_{r}$ Buurman WA, Blanco $C$. Intestinal permeability and carrier-mediated monosaccharide absorption in preterm neonates during the early postnatal period. Pediatr Res 2002;51:64-70.

37. de Jaeger A, Proulx F, Yandza T, Dugas M-A, Bioeuf $B$, Manika A, Lacroix J, Lambert M. Markers of cellular dysoxia during orthotopic liver transplantation in pigs. Intensive Care Med 1998;24: 268-75. 
38. Englund $R$, Lalak. $N_{i}$ Jacques $T$, Hanel $K C$. Sigmoid anid gastric tonometry during infrarenal aortic aneurysm repair. Aust N ZJ Surg 1996;66(2):88-90.

39. Andersen $L W$, Landow $L$, Baek $L$, Jansen $E_{r}$ Baker $S$. Association between gastric intramucosal $\mathrm{pH}$ and splanchnic endotoxin, antibody to endotoxin, and tumor necrosis factor-a concentrations in patients undergoing cardiopulmonary bypass. Crit Care Med 1993;21 (2):210-7.

40. Handley DA, Tomesch JC, Saunders RN. Inhibition of PAF-induced systemic responses in the rat, guinea pig, dog and primate by the receptor antagonist SRI 63-441. Thromb Haemost $1986 ; 56(1): 40-4$.

41. Farquhar I, MartinCM, Lam C, Potter R, Ellis CG, Sibbald WJ. Decreased capillary density in vivo in bowell mucosa of rats with normotensive sepsis. I Surg Res 1996;61:190-6.

42. Bersten $A D$, Hersch $M$, Cheung $H$, Rutlledge FS, Sibbald WI. The effect of various sympathomimetics on the regional circulations in hyperdynamic sepsis. Surgery $1992 ; 112(3)$ : 549-61.

43. Bersten $A D$, Hersch $M$, Cheung $H$, Rutledge FS, Sibballd WJ. The effect of various sympathomimetics on the regional circulations in hyperdynamic sepsis. Surgery 1992;112(3): 549-61.

44. Lam $C_{x}$, Tyml $K_{n}$. Martin $C_{r}$ Sibbald WJ. Microvascular perfusion is impaired in a rat model of normotensive sepsis. I Clin Invest 1994;94:2077-83.

45. Silbbald $W$, Fox $G_{i}$, Martin $C$. Abnormalities of vascular reactivity in the sepsis syndrome. Chest 1991;100(3):1555-9S.

46. Douzinas EE, Tsidemiadou PD, Pitaridis MT, Andrianakis I, Bobota-Chloraki A, Katsouyanni K, Sfiyras $D$, Mallagari $K$, Roussos $C$. The regional production of cytokines and lactate in sepsisrelated multiple organ failure. Am I Respir.Crit Care Med 1997;155:53-9.

47. Poeze $M$, Froon AHM, Ramsay $G$, Buturman WA, Greve JWM. Decreased organ failure in patients with severe SIRS and septic shock treated with the platelet-activating factor antagonist TCV-309: a prospective, multicenter, double-blind, randomized phase II trial. TCV-309 Septic Shock Study Group. Shock 2000; 14(4):421-8.

48. Ferraz $\mathrm{JG}_{\mathrm{f}}$, Tigley $\mathrm{A}$, Wallace $\mathrm{JL}$. Paradoxical effects of $\mathrm{L}$-arginine on gastric mucosal integrity. Eur ) Pharmacol 1994,260(1):107-11.

49. Kilbourn $\mathrm{RC}$, Szabo C, Traber DL. Beneficial versus detrimental effects of nitric oxide synthase inhibitors in circulatory shock: lessons learned from experimental and clinical studies. Shock $1997,7(4): 235-46$.

50. Gundersen $Y_{\text {, Corso }} C_{r}$ Leiderer R., Lilleaasen P, Aasen AO, Messmer $K$. Use of selective and nonselective nitric oxide symthase inhibitors in rat endotoxemia: effects on hepatic morphology and function. Shock 1997; 8(5):368-72.

51. Minnard EA, Shou J, Naama $H$, Cech A, Gallagher $H$, Daly JM, Inhibition of nitric oxide synthesis is detrimental during endotoxemia. Arch Surg 1994;129(2):142-7.

52. Harbrecht $B G$, Wu $B$, Watkins $C$, Billiar TR, Peitzman AB. Inhibition of nitric oxide synthesis during severe shock but not after resuscitation increases hepatic injury and neutrophil accumulation in hemorrhaged rats. Shock 1997;8(6):415-21.

53. Poeze M, Bruins MI. Arginine supplementation during sepsis: from theory to practise. In: Vincent J$L$ (ed).Yearbook of Intensive Care and Emergency Medicine. 1st ed. Berlin: Springer Verlag; 2003; 97-106. 
54. Luiking $Y C$, Steens $L$, Poeze $M_{\text {, }}$ et al, Low plasma arginine concentration in septic patients is related to diminished de novo arginine production from citrulline. [Abstract] Clin Nutr 2003,22: S26.

55. Gutierrez G, Palizas F, Doglio G, Wainsztein N, Gallesio A, Pacin J, Dubin A, Schiavi E, Jorge $\mathrm{M}$, Pusajo J, et al. Gastric intramucosal pH as a therapeutic index of tissue oxygenation in critically ill. Lancet 1992;339(1):195-9.

56. Ivatury RR, Simon RJ; "slam SI, Fueg A, Rohman M, Stahl WM, A prospective randomized study of end points of resuscitation after major trauma: global oxygen transport indices versus organspecific gastric mucosal pH. I Am Coll Surg 1996/183:145-54.

57. Barquist E, Kirton O, Windsor $\mathrm{g}$, Hudson-Civetta , Lynn M, Herman M, Civeta J. The impact of antioxidant and splanchnic-directed therapy on persistent uncorrected gastric mucosal pH in the critically injured trauma patient I Trauma 1998;4(2):355-60.

58. Spies CD, Reinhart $K$, Witt $I$, Meier-Hellmann A, Hannemann $L$, Bredle DL, Schaffartzilk W. Influence of $\mathrm{N}$-acetylcysteine on indirect indicators of tissue oxygenation in septic shock patients: results from a prospective, randomized, double-blind study, Crit Care Med 1994;22(11):1738-46.

59. Gomersall CD, Joynt GM, Freebairn RC, Hung V, Buckley TA, Oh TE, Resuscitation of critically ill patients based on the results of gastric tonometry: a prospective, randomized, controlled trial. Crit Care Med 2000;28(3):607-14. 
CHAPTER 15

Samenvalling 
Dit proefschrift heeft betrekking op het klinische beeld sepsis en meervoudig organ falen. Sepsis wordt gedefinieerd als 'de algehele lichamelijke gevolgen van een infectie'. Wanneer als uiting van ernstige sepsis meerdere organen slecht functioneren wordt dit meervoudig orgaan falen (multiple organ failure (MOF)) genoemd. Patiënten met sepsis, die op de intensive care opgenomen worden hebben een verhoogde kans op het ontwikkelen van ernstige complicaties en een verhoogde kans om te sterven. Per jaar overlijden er ongeveer 3500 mensen in Nederland aan de gevolgen van sepsis. Gemiddeld is het overlevings-percentage van patiënten met sepsis 50-70\%. Dit percentage wordt voornamelijk bepaald door het falen van orgaanfuncties.

Eén van de kenmerken die patienten met orgaan falen ten gevolge van sepsis hebben, is een gestoorde doorbloeding door deze organen. Ook chirurgische patiënten met een verhoogd risico op het ontwikkelen van sepsis en orgaan falen blijken deze doorbloedingsstoornissen reeds te hebben. Alhoewel uit één van onze eerdere onderzoeken blijkt dat intensivisten het moeilijk vinden om deze kenmerken valn sepsis te detecteren, wordt het bestaan van deze afwijkingen wel belangrijk gevonden. Dit en de hoge sterftekans, dat met sepsis en MOF gepaard gaat, is de reden geweest om deze onderzoeken te verrichten. In dit proefschrift zijn een aantal methoden om de verstoorde doorbloeding te meten, onderzocht. De gebruikte methoden zijn de Doppler ultrasone techniek, het meten van D-lactaat in het bloed, het meten van de kooldioxide-productie in het darmslijmvlies en het meten van de totale lever-darmdoorbloeding middels de indocyanine groen-verdunningsmethode. Verder zijn een aantal methoden gezocht om deze doorbloedingssttoornissen te behandelen. Twee van de hoofdstukken gaan in op het verbeteren van de doorbloeding middels dopexamine, een hartversterkend middel, waarvan gedacht wordt dat het ook speciaal de doorbloeding door het lever-darm gebied kan verbeteren. De laatste twee hoofdstukken gaan in op de invloed van stikstofoxide (NO) op de doorbloeding en het functioneren van de lever en darm tijdens sepsis. Hierbij zijn zowel medicijnen gebruikt die een remmende als een stimulerende invloed op het onstaan van NO hebben.

In hoofdstuk 4 wordt het belang van niet opgemerkte doorbloedingsstoornissen bij patiënten benadrukt. In dit onderzoek werd de voorspellende waarde op het ontwikkelen van post-operatieve complicaties, van afwijkende vullingsvolumes van het hart bij hoog-risico chirurgische patiënten, zoals gevonden met behulp van een ultrasoon Doppler onderzoek, vergeleken met de standaard bewakingstechnieken. Het bleek dat deze patiënten, bij wie een gestoordle doorbloeding en vulling gevonden werden mbv. het ultrasoon Doppler onderzoek een verhoogde kans op het ontwikkelen van complicaties hadden. Bovendien konden deze afwijkingen vaak niet gevonden worden met de standaard bewakingstechnieken.

In het volgende hoofdstuk wordt een onderzoek beschreven waarbij dergelijke hoog-risico chirurgische patiënten rondom de operatie behandeld werden om dit risico op complicaties te verkleinen (hoofdstuk 5). Daarbij werd met behulp van het geven van extra infusievloeistoffen en een hartversterkend middel, dopexamine, gepoogd een dergelijke gestoorde doorbloeding en vulling te behandelen voordat 
deze complicaties zouden veroorzaken. Het bleek dat het aantal complicaties en het sterfte percentage niet daalden in de totale groep, maar wel in een subgroep van patiënten met het hoogste risico (bv. patiënten die een spoedoperatie moesten ondergaan).

Bij het ontwikkelen van MOF spelen doorbloedingsstoornissen door de lever en de darm een belangrijke rol. Een van de compensatoire reacties op verminderde vulling en doorbloeding van het hart is de doorbloeding door de lever en de darm te verminderen, zodat een groter gedeelte van het volume door het hart kan gaan. Als deze situatie echter te lang duurt kan een verslechterde doorbloeding door de lever en de darm op zichzelf aanleiding geven tot het ontwikkelen van meervoudig orgaan falen. In de volgende studie werd het belang van het meten van de algemene (globale) doorbloeding vergeleken met het meten van doorbloeding en functie van de lever en darm bij patiënten met sepsis en orgaan falen (hoofdstuk 7). Bij opname op de intensive care van deze patiënten met een lage bloeddruk (shock) bleken beide meetmethoden even belangrijk te zijn in het voorspellen van de overlevingskans. $\mathrm{Na}$ behandeling van de lage bloeddruk (shock) bij deze patiënten, bleek de voorspellende waarde tav. de overlevingskans van het meten van de lever en darmdoorbloeding groter te zijn dan die van de globale doorbloeding.

In de volgende studie werd de waarde van D-lactaat bepaald als marker van het optreden van een slechte doorbloeding (ischemie) door de darm (hoofstuk 8). D-lactaat is een stof, die in de darm geproduceerd wordt door de darmbacteriën als de darm te weinig bloedtoevoer krijgt. Het bleek dat het meten van het D-lactaat vlak na een spoedoperatie in verband met een gesprongen buikslagader een goede voorspelling gaf van het optreden van ischemie van de darm. In een ander onderzoek bleek de hoogte van dit D-lactaat gerelateerd te zijn aan de mate van zuurstofvoorziening van de darm bij patiënten met sepsis en MOF (hoofdstuk 9).

In hoofdstuk 10 wordt de mate van zuurstofvoorziening van de darm bij hoogrisico chirurgische patiënten, middels tonometrie van het magsslijmvlies, geëvalueerd. Deze methode meet het kooldioxide $\left(\mathrm{CO}_{2}\right)$, dat geproduceerd wordt gedurende de normale stofwisseling in het slijmwlies van de darm. In het algemeen geldt: hoe slechter de doorbloeding, hoe meer kooldioxide geproduceerd wordt. In deze studie bleek dat bij hoogrisico chirurgische patiëntengroep een hogere maagslijmvlies $\mathrm{CO}_{2}$ waarde het optreden van MOF kon voorspellen. Deze patiënten hadden tevens een grotere kans op overlijden ten gevolge van de operatie.

In hoofdstuk 11 wordt ingegaan op de relatie tussen doorbloedingsstoornissen in de lever en de darm en de mate van ontstekingsreactie in dit gebied. In deze studie wordt beschreven hoe patiënten die uiteindelijk MOF ontwikkelen al vroeg na de operatie zowel een slechtere doorbloeding in het lever-darm gebied hebben als een toegenomen onstekingsreactie in het lever-darm gebied vertonen.

De afsluitende studies (hoofdstuk 12 en 13) van dit proefschrift gaan over het beïnvloeden van de doorbloeding en ontstekingsreactie in het lever-darm gebied door het beïnvloeden van het stikstofoxide (NO (nitric oxide)). Dit NO speelt 
een belangrijke rol in het ontstaan van de bloeddrukdalingen bij sepsis door de vaatverwijding die het veroorzaakt. Aan de andere kant zorgt deze vaatverwijding ook voor een behoud van doorbloeding in de kleinste bloedvaatjes in het lever-darm gebied tijdens bloeddrukdalingen. Aanvankelijk werd aangenomen dat remming van dit NO voordelig zou zijn voor de septische patiënt met bloeddrukdalingen. Ons onderzoek bij varkens toont echter aan dat het geven van een remmer van de NO productie niet voordelig is tijdens sepsis. Tevens toonde een ander experiment aan dăt het geven van arginine, een van de voorlopers van NO, voordelig is tijdens sepsis.

260 Concluderend kan gesteld worden dat bij patiënten met een verhoogd risico op het ontwikkelen van meervoudig orgaan falen het belangrijk is om met zo min mogelijk invasieve bewakingsmethoden de globale doorbloeding te optimaliseren om zo het risico op meervoudig orgaan falen en daarmee de sterftekans te reduceren. Bij patiënten die reeds meervoudig orgaan falen hebben ontwikkeld, is het meten van de globale doorbloeding niet meer voldoende om stoornissen in doorbloeding te detecteren, maar zal bij de bewaking van de doorbloeding ook de lever- en darm-doorbloeding moeten meten. Afwijkingen in deze metingen zijn gerelateerd aan de overlevingskans. Er zijn aanwijzingen dat correctie van deze afwijkingen een betere overlevingskans kan geven bij patiënten met meervoudig orgaan falen. 


\section{LIST OF PUBLICATIONS}

\section{Studies presented in this thesis}

M Poeze, AHM Froon, IWM Greve, G Ramsay. D-lactate as an early marker of intestinall ischemia after abdominal aneurysm repair. Br J Surg 1998; 85:1221-1224.

M Poeze, G Ramsay, IWM Greve, M Singer. Prediction of postoperative cardiac surgical morbidity and organ failure within 4 hours of ICU admission using esophageal Doppler ultrasonography. Crit Care Med 1999; 127:1288-1294.

J Takala, A Meier-Hellmann, I Eddleston, et al., on behalf of the European Multicenter study group on dopexamine in major abdominal surgery. Effects of dopexamine on outcome after major abdominal surgery: a prospective, randomized, controlled multi-center study. Crit Care Med 2000; 28:3417-3423

M Poeze, J Takala, JWM Greve, G Ramsay, on behalf of the European Multicenter study group on dopexamine in major abdominal surgery. Pre-operative tonometry is predictive for mortality and morbidity in high-risk surgical patients. Intensive Care Med 2000; 26:1272-1281

M Poeze, G Ramsay, WA Buurman, JWM Greve, I Takala. Increased hepatosplanchnic inflammation precedes the development of organ failure aiter elective high-risk surgical patients. Shock, 2002; 17:451-458.

M Poeze, $\mathrm{BCl}$ Solberg, JWM Greve, G Ramsay. Gastric $\mathrm{PgCO}_{2}$ and $\mathrm{Pg}-\mathrm{aCO} \mathrm{CO}_{2}$ gap are related to D-lactate and not to L-lactate levels in patients with septic shock. Intensive Care Med 2003;29(2):2081-2085.

M Poeze, B Solberg, JWM Greve, G Ramsay. Monitoring globall haemodynamic or regional parameters in critically ill patients: what is more important during intensive care resuscitation. Accepted for publication in: Crit Care Med.

M Poeze, MJ Bruins, IJH Vriens, et al. Selective nitric oxide synthase-2 inhibition during prolonged porcine endotoxemia increases mortality and deteriorates hepatosplanchnic perfusion and metabolism. Submitted.

M Poeze, MJ Bruins, G Ramsay, et al. Effects of L-arginine supplementation as pretreatment to porcine endotoxemia on nitric oxide metabolism and hepatosplanchnic perfusion. Submitted.

M Poeze, JWM Greve, G Ramsay. A meta-analysis of haemodynamic optimisation of peri-operative patients and patients with sepsis: relationship with the methodological trial quality. Submitted. 


\section{Other papers}

M Poeze, JGA Houbiers, CJH van de Velde, Th Wobbes, MF von Meyenfeldt. Results of radical resection of locally advanced colorectal carcinoma. Br I Surg 1995;82:367-371.

M Poeze, MF von Meyenfeldt, IL Peterse, WD George, JMA de Jong, Jl Jager, FBJM. Thunnissen. Increased proliferation and p53 expression in nonmalignant breast tissue after radiation therapy. I Pathol 1998; 185;32-37.

M Poeze, JWM Greve; G Ramsay. Geen meerwaarde van lactaatplasmabepaling bij patiënten met acute buik [brief]. NTvG 1998; 142:1346-1347.

M Poeze, JWM Greve, $G$ Ramsay. Oxygen delivery and septic shock [letter]. Chest $1999 ; 116: 1145$.

M Poeze, B Tjaandra, G Freling, MHJM Herpers. Spinal teratoma: case report and review of the literature. Neurosurgery 1999; 45(2):379-385.

M Poeze, JWM Greve, G Ramsay. Goal-oriented haemodynamic therapy: a plea for a closer look at using peri-operative oxygen transport optimisation. Intensive Care Med, 2000; 26(5): 635-637.

M Poeze, AHM Froon, G Ramsay, WA Buurman, JWM Greve, and the TCV-309 Septic Shock Study Group. Decreased organ failure in patients with severe SIRS and septic shock treated with the platelet-activating factor antagonist TCV-309. Shock 2000; 14:421-428.

MRM Scheltinga, M Poeze, M de Haan, JHM Tordoir, PJEHM Kitslaar. Prostetic femorocrural bypass surgery and adjuvant arteriovenous fistulae. Ann Vasc Surg 2003;17(2):203-209.

M Poeze, G Ramsay. Monitoring ICU patients: global and regional aspects. Neth I Crit Care Med 2003; 7(6): 373-378

M Poeze, G Ramsay, H Gerlach, M Levy. An International Sepsis Survey: A Study of Doctors' Knowledge and Perception about Sepsis. Submitted. 


\section{Book chapters}

M Poeze, JWM Greve, G Ramsay. Necrotizing fasciitis. In: JVincent (ed.). Yearbook of Intensive Care and Emergency Medicine, Springer Verlag, Berlin Heidelberg, 1996, pp.533-541.

M Poeze, G Ramsay. Prognostic factors in pre-septic states. In: IL Vincent (ed.). Yearbook of Intensive Care and Emergency Medicine, Springer Verlag, Berlin Heidelberg, 1997, pp.161-167.

M Poeze, JWM Greve, G Ramsay. Is splanchnic perfusion critical in sepsis? In: AE. Baue, G Berlot, A Gullo, IL Vincent (eds), Sepsis and organ dysfunction. From basics to clinical approach. 1998, 1 st ed , ch. 17, pp.169-180.

M Poeze, G Ramsay. Management of abdominal injury. In: AR Webb, MJ Shapiro, M Singer, PM Suter (eds.). Oxford Textbook of Critical Care, 1999, Oxford University Press, London, 1st ed. , ch. 11.1.5, pp.719-721.

M Poeze, IWM Greve, G Ramsay. The surgical abdomen. In: JF Bion (ed.). Intensive Care Medicine. Series eds. RM Jones, AR Aitkenhead, Foëx P. Fundamentals of Intensive Care, 1999, BMJ Books, Londion, 1st ed., ch. 17, pp. 203-212.

M Poeze, G Ramsay, JWM Greve. Platelet-Activating Factor. In: Baue, Fry (eds). SIRS, MODS and MOF - Systemic inflammatory response syndrome, multiple organ dysfunction syndrome and multiple organ failure - pathophysiology, prevention and therapy. 2000, Springer Verlag, Berlin, 1st ed., ch. 22, pp. 198-102.

G Ramsay, M Poeze, IS Grant, C Wallis, A Cushieri. Management of critically ill patients. In: A Cushieri, GR Gilles, AR Moossa (eds.). Essential surgical practice. 2000, Butterworth Heinemann, Oxford, 4th ed.,19, pp. 427-456.

M Poeze, IWM Greve, G Ramsay. Gut decontamination - prevention of translocation. In: Baue, Fry (eds). SIRS, MODS and MOF - Systemic inflammatory response syndrome, multiple organ dysfunction syndrome and multiple organ failure - pathophysiology, prevention and therapy. 2000, Springer Verlag, Berlin, 1 st ed., ch. 59, pp. 580-590.

M Poeze, G Ramsay. Monitoring intensive care patients: global and regional aspects. In: IL Vincent (ed). Yearbook of Untensive Care and Emergency Medicine, Springer Verlag, Berlin Heidelberg, 2002, pp. 612-631.

M Poeze, MJ Bruins. Supplementing L-arginine during sepsis: from theory to clinical practise. In: $J L$ Vincent (ed). Yearbook of Intensive Care and Emergency Medicine, Springer Verlag, Berlin Heidelberg, 2003, pp. 97-106. 


\section{Citated abstracts}

M Poeze, G Ramsay. Anti endotoxin immunotherapy. Does it work? Proceedings of the 8th European Congres of Intensive Care Medicine, 1995;8:441-447.

M Poeze, J Rapon, JWM Greve, G Ramsay. Use of shock index as prognostic factor in ICU-patients with SIRS. Br I Surg, 1997;84: 876-877.

264 M Poeze, I Rapon, JWM Greve, G Ramsay. Incildence of SIRS and organ failure on a mixed ICU - The definitions evaluated. Br J Surg, 1997; 84: 880-881.

M Poeze, AFM Froon, JWM Greve, G Ramsay. D-lactate as a marker for intestinal ischemia after abdominal aneurysm repair. $\mathrm{Br} / \mathrm{Surg}$. 1997; 84: 873-874.

MPoeze, GRamsay, JWM Greve, M Singer. Stroke volume by esophageal Doppler is a predictor of postoperative morbidity, in contrast to cardiac output. Intensive Care Med. 1997; 23 (Suppl 1): S83.

M Poeze, G Ramsay, JWM Greve, I Takala. Shoemaker criteria as prognostic marker for postoperative morbidity and mortality. Br J Surg 1998; 85:861.

M Poeze, G Ramsay, JWM Greve, M Singer. Is stroke volume a better marker than cardiac output for predicting postoperative morbidity? Br \Surg 1998; 868-869.

M Poeze, I Takala, JWM Greve, G Ramsay. Peri-operative responses in regionall and global hemodynamic perfusion to dopexamine is variable depending on pre-operative gastric pHi value. Intensive Care Med 1998; 24 (Suppl 1):S132

M Poeze, JWM Greve, WA Buurman, JTakala. Peri-operative hepatosplanchnic cytokine production is related to postoperative hepatic fallure and to regional perfusion. Intensive Care Med 1998; 24 (Suppl 1):S132.

M Poeze, AHM Froon, G Ramsay, WA Buurman, JWM Greve, and the TCV-309 Septic Shock Study Group. Organ fallure and MOF-related death is reduced in patients with septic shock treated with the PAF-antagonist TCV-309. Intensive Care Med 1998; 24 (Suppl 1):S69.

M Poeze, G Ramsay, JWM Greve, J Takala. Shoemaker criteria as prognostic marker for postoperative morbidity and mortality. Intensive Care Med 1998; 24:40.

S Manders, M Poeze, G Ramsay, JWM Greve, NEP Deutz. Plasma nitrate in surviving patients with septic shock are increased compared to non-survivors. Intensive Care Med 1999; $25:$ :S6. 
M Poeze, WA Butrman, IWM Greve, G Ramsay, Sustained SIRS: Increased inflammation and predictor of outcome, but not useful in individual patients. Intensive Care Med 1999; 25:\$124.

M Poeze, PPGM Kouwenberg, G Ramsay, et al. Patients with HLA-DR6 serotype and multiple organ failure have increased levels of TNF-, LBP, and BPI. Intensive Care Med 1999; 25:S102.

M Poeze, M Bruins, I Vriens, G Ramsay, NEP Deutz. Influence of selective iNOS inhibition on hepatosplanchnic function and survival during procinne endotoxaemia. Intensive Care Med 2000; 26:5344.

M Poeze, B Solberg, JWM Greve, G Ramsay. Gastric pHi and $\mathrm{PrCO}_{2}$ are related to D-lactate and not to L-lactate levels. Intensive Care Med 2000; $26: 5343$.

M Poeze, B Solberg, IWM Greve, G Ramsay. Procalcitonon levels predict decrease in $\mathrm{pHi}$ despite increase in oxygen delivery during resuscitation. Intensive Care Med 2000; 26:\$228.

BC) Solberg, M Poeze, R Trompmeesters, G Ramsay. Tonometric variables remain unchanged during dexmedetomidine infusion. Intensive Care Medicine 2000; 26 : S348.

$K$ Barendregt, M Poeze, PB Soeters. The influence of sepsis on bioelectrical impedance spectroscopy in pigs. Clin Nutrition 2000;19 (Suppl 1):6.

M Poeze, MJ Bruins, MJ Vriens, G Ramsay, NE Deutz. Selective iNOS inhibition decreases in vivo NO production, but increases mortality during porcine endotoxaemia. Intensive Care Med 2001; 27:S243.

M Poeze, BC Solberg, JW Greve, G Ramsay. Monitoring regional versus global haemodynamic parameters in critically ill patients: what is better? Intensive Care Med $2001 ; 27: 5271$.

YC Luiking, L Steens, M Poeze, et al. Low plasma arginine concentration in septic patients is related to diminished de novo arginine production from citrulline. Clin Nutr $2003 ; 22: 526$. 


\section{DANKWOORD}

Het is af en daar gaan we een goede borrel op drinken. Dat afkrijgen van het boekje is iets dat je uiteindelijk in je eentje moet doen, maar er waren een boel mensen die me een flink eind op weg geholpen hebben. Als ik al die mensen moet bedanken wordt het boekje nog dikker dan het al is, dus vergeef het me als ik je niet persoonilijk vermeld.

Prof. dr. Ramsay, beste Graham. Ik heb vaak op je staan schelden als het weer eens maanden duurde voordat je mijn manuscripten nagekeken had. Je bent echter absoluut een stimulerende persoon geweest. Als ik zie hoeveel kansen ik door jou heb gekregen (schrijven hoofdstukjes, uitnodigingen voor praatjes op de "grote" congressen), dan ben ik daar zeer dankbaar voor. Het blijft eeuwig jammer dat je uit de kliniek weg bent, want ik had graag nog het klinische werk van je geleerd. Verder kon ik de 'sociale activiteiten' erg waarderen; een biertje drinken als je al ziek en beroerd bent, is nog steeds een goede remedie.

Dr. Greve, beste Jan Willem, dank je wel voor je begeleiding. Alhoewel de begeleiding op een gegeven moment wat meer vanaf de zijlijn plaatsvond, heb ik je commentaren op de manuscripten altijd als zeer gedegen ervaren. Minutieus werden alle fouten eruit gehaald. Bedankt daarvoor. Als je me straks in Maastricht met dezelfde gedegenheid en enthousiasme het klinische werk onderwijst, zal ik tevreden zijn.

Prof. dr. Buurman, beste Wim, als we weer eens over een artikel discussieerden, werd er meestal van alles bijgehaald. Via de salarissen en andere misstanden in de gezondheidszorg kwam je dan uiteindelijk toch altijd weer op de kern van de artikel uit en kon je haarfijn uitleggen waarom het allemaal niet klopte wat ik opschreef. Helaas ben je wat meer op de achtergrond gekomen, maar ik heb je inzet gewaardeerd.

Dr. Deutz, beste Mick, alhoewel pas laat betrokken bij mijn proefschrift heb je een niet onbellangrijke rol gespeeld. Je enthousiasme is zeer aanstekelijk en de discussies waarbij we die verschrikkelijke grote hoeveelheid aan data doorwerkten waren zeer inspirerend. $\mathrm{k}$ denk dat we elkaar de komende tijd nog veel zullen tegenkomen. Bedankt dat je zitting hebt willen nemen in de corona.

De leden van de beoordelingscommissie: Prof. dr. Leunissen, dr. Marcus, Prof. dr. Radermacher en Prof. dr. Singer ben ik zeer erkentelijk voor het feit dat zij dit boekwerk wilden doorploeteren en beoordelen.

Prof. dr. Radermacher, dear Peter. Thank you for feeling honored participating in the thesis committee. I have very much appreciated the openness with which you shared your ideas and study data. If more people were like you the scientific world would be more fruitful in achieving its goals. Prof. dr. Singer, dear Mervyn. That's what I call enthusiasm! I have always been surprised about your energy and I am glad that you were willing to participate in the thesis committee. Thanks you for letting this strange Dutch guy stay at your institution. 
De collega-promovendi, Dennis, Steven, Karel, Roland, Ellen en Maaike, van whe sommigen nog bezig en anderen al lang klaar zijn, wil ik ook bedanken voor de gezellige uren pratend over de ellende wan het promoveren: achteraf is het zo erg nog niet. Met name Dennis bedankt voor al die zweterige uurtjes in het kleinste hokje van het ziekenhuis ("Is dit de keuken?").

'Ondersteunend personeel' noemen ze dat dan. Nou zonder jullie komt er helemaal niets van zo'n boekje terecht. Op het lab ben ilk eeuwige dank verschuldigd onder andere aan Trudy Jeunhomme, Mieke Dentener, Hans van Eijk en Gabrie ten Have.

Barbara Solberg neemt hierbij een aparte plaats in. Inmiddels niet meer op de IC te vinden, maar zonder luisterend oor en onderzoeksmaatje was het niet half zo leuk geweest. Over IC personeel gesproken; jullie hebben me nogal eens vervloekt als ik weer met mijn metingen en bloedmonsters ("vampier') langskwam. Nam de mortaliteit nu toe als ik langs geweest was? Toch hebben jullie met je kritische vragen en hulp aanzienlijk bijgedragen aan het voltooien van de studies.

Mijn collega assistenten en bazen in het 'Diac' wilde ik bedanken voor het gedulld dat ze hadden als ik weer eens een uitwijding begon over de wetenschap. Hopelijk heb ik wat van mijn enthousiasme voor het onderzoek op jullie kunnen overbrengen.

Mijn paranimfen, Robert en Erwin ('wat lijken we op elkaar, nietwaar"); wat fantastisch om jullie als broer en paranimf te hebben, waarvoor mijn dank. Eindelijk zie ik jullie in een net pak. Dit bedankje geldt dan natuurlijk ook voor paps en mams, die hun zoon frequent niet hebben gezien en die vaak het een en ander vergat. Bedankt voor het geduld.

Als een na laatste Loes. Je wilt eigenlijk niet dat ik hier allemaal bedankjes opschrijf en al helemaal niet met koosnaampjes en al erbij. Overigens terecht, dus. hou ik het bij bedankt. De inhoud hiervan weet je wel.

Als laatste mijn dank aan alle familieleden en patiënten, die toestemming gaven voor het meedoen aan de studies. Zonder uw moed in een moeilijke tijd is het niet mogelijk om ooit maar enige vooruitgang te boeken in het behandelen van sepsis en orgaan falen. 


\section{CURRICULUM VITAE}

Martijn Poeze was born on the $11^{\text {th }}$ of August 1971 in Muiden. After attending high school (Atheneum) at the Willem de Zwijger College in Bussum, he studied medicine at Maastricht University from 1989. In 1997 he graduated from medical school. During the last two years, he also worked as a ambulance paramedic in the South-eastern part of Limburg.

During his medical studies he spent half a year in Rio de Janeiro, Brasill, for an elective in Obstetrics and performed research at the department of surgery studying the prognostic value of locally advanced colorectal carcinoma and the effects of radiation therapy on normal breast tissue, the latter at the University of Glasgow (head prof. dr. W.D. George).

After the first year of clerkships, he became a research fellow at the department of surgery. After graduation from medical school the fellowship was continued. Part of the research was performed at the intensive care of the University College London, guided by Prof.dr. M. Singer and Kuopio University, Finland, guided by Prof. dr. J. Takala. The results of the studies performed are presented in this thesis. For the research performed at the University College in London he was awarded the Travelling Fellowship Award of the Surgical Infection Society. For his work on hepatosplanchnic metabolism and inflammation he was awarded the Young Investigator Award of the European Society of Intensive Care Medicine in 2000 and the British Journal of Surgery Research Bursary in 2002.

In 2000 he started his surgical training at the Máxima Medical Center in Eindhoven (head: dr. J.W. Prakken). He currently lives in Eindhoven with Loes van Bokhoven, whom he married in 2002. In december 2004 his son Jelle was born. 